Peter Gottfried

\title{
Die verdeckten \\ Effizienzwirkungen der Umsatzsteuer
}




\section{Peter Gottfried}

\section{Die verdeckten Effizienzwirkungen der Umsatzsteuer}

Über das Vorsteuerabzugsverfahren wird erreicht, daß im Endeffekt nur die privaten Konsumausgaben von der Umsatzsteuer betroffen sind. In der Regel erlischt jedoch das Vorsteuerabzugsrecht für steuerbefreite Unternehmen. Damit wird die Kette des Vorsteuerabzugs unterbrochen, wenn immer ein Gut im Zuge seiner Herstellung eine befreite Produktionsstufe durchläuft. Die vorliegende Arbeit untersucht die allokativen Konsequenzen dieser speziellen Form der Steuersatzdifferenzierung im Rahmen eines Empirischen Allgemeinen Gleichgewichtsmodells für die Bundesrepublik Deutschland. Zur besseren ökonomischen Interpretation der Ergebnisse wird das ebenfalls in der Arbeit entwickelte Konzept der effektiven Steuersätze herangezogen.

Peter Gottfried wurde 1959 in Heidelberg geboren. Er studierte von 1980 bis 1986 Volkswirtschaftslehre an den Universitäten Heidelberg und Canterbury. Seit 1987 ist er Wissenschaftlicher Mitarbeiter am Lehrstuhl für Finanzwissenschaft der Universität Regensburg und promovierte dort 1991 zum Dr. rer. pol. 
Die verdeckten Effizienzwirkungen der Umsatzsteuer Eine Empirische Allgemeine Gleichgewichtsanalyse 


\section{FINANZWISSENSCHAFTLICHE SCHRIFTEN}

Herausgegeben von den Professoren Albers, Krause-Junk, Littmann, Oberhauser, Pohmer, Schmidt

Band 47

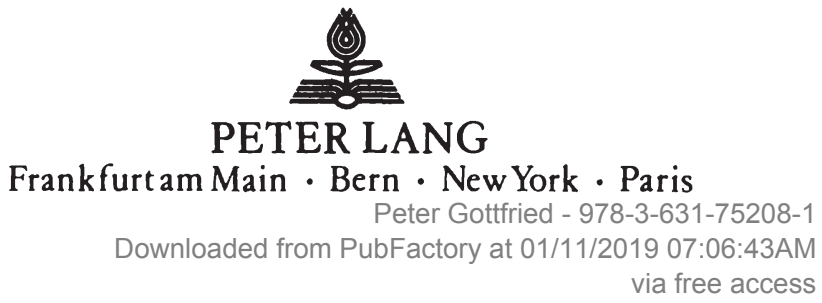




\section{Peter Gottfried}

\section{Die verdeckten Effizienzwirkungen der Umsatzsteuer}

Eine Empirische Allgemeine

Gleichgewichtsanalyse

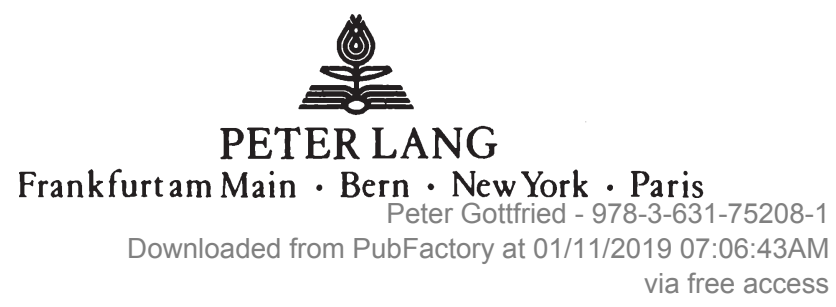


Die Deutsche Bibliothek - CIP-Einheitsaufnahme

Gottfried, Peter:

Die verdeckten Effizienzwirkungen der Umsatzsteuer : eine empirische allgemeine Gleichgewichtsanalyse / Peter Gottfried.

- Frankfurt am Main ; Bern ; New York ; Paris : Lang, 1992

(Finanzwissenschaftliche Schriften ; Bd. 47)

Zugl.: Regensburg, Univ., Diss., 1991

ISBN 3-631-44210-6

NE: GT

Open Access: The online version of this publication is published on www.peterlang.com and www.econstor.eu under the international Creative Commons License CC-BY 4.0. Learn more on how you can use and share this work: http://creativecommons. org/licenses/by/4.0.

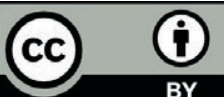

This book is available Open Access thanks to the kind support of ZBW - Leibniz-Informationszentrum Wirtschaft.

\author{
D 355 \\ ISSN 0170-8252 \\ ISBN 3-631-44210-6 \\ ISBN 978-3-631-75208-1 (eBook) \\ cc Verlag Peter Lang GmbH, Frankfurt am Main 1992 \\ Alle Rechte vorbehalten.
}

Das Werk einschließlich aller seiner Teile ist urheberrechtlich geschūtzt. Jede Venwertung außerhalb der engen Grenzen des Urheberrechtsgesetzes ist ohne Zustimmung des Verlages unzulāssig und strafbar. Das gilt insbesondere fūr Vervielfältigungen, Übersetzungen, Mikroverfilmungen und die Einspeicherung und Verarbeitung in elektronischen Systemen. 
Für

Helga,

Daniela

und

meine Eltern

Peter Gottfried - 978-3-631-75208-1

Downloaded from PubFactory at 01/11/2019 07:06:43AM

via free access 
Peter Gottfried - 978-3-631-75208-1

Downloaded from PubFactory at 01/11/2019 07:06:43AM

via free access 


\section{Vorwort}

Die vorliegende Arbeit entstand während meiner Tätigkeit als Wissenschaftlicher Mitarbeiter am Lehrstuhl für Finanzwissenschaft der Universität Regensburg. Sie baut insbesondere auf dem in Zusammenarbeit mit Herrn Prof. Dr. Wolfgang Wiegard verfaßten Artikel ,Exemption versus Zero Rating: A Hidden Problem of VAT auf, der in Kürze im Journal of Public Economics erscheint. Erste Ergebnisse wurden bereits in Brunner/Petersen [1990] ,Simulation Modells in Tax and Transfer Policy' veröffentlicht.

Die Arbeit wurde im Mai 1991 von der Wirtschaftswissenschaftlichen Fakultät der Universität als Promotionsschrift angenommen.

Mein Dank gilt in erster Linie Herrn Prof. Dr. Wolfgang Wiegard, der mir hervorragende Arbeitsbedingungen zugestand und mich in allen, auch nicht fachbezogenen Belangen tatkräftig unterstützte. Desweiteren danke ich Herrn Prof. Dr. Wolfgang Buchholz für seine Bereitschaft zur Mitarbeit in der Betreuungskommission und für seine wertvollen Anregungen und Verbesserungsvorschläge. Als sehr hilfreich erwiesen sich immer die Diskussionen mit Herrn Dr. Elmar Stöß. Eine gewissenhafte und auch kritische Durchsicht des Manuskripts übernahmen dankenswerterweise Herr M.A. Econ. Christoph Rosenberg und Herr Diplom-Volkswirt Hans Fehr.

Für noch verbliebene Unrichtigkeiten ist selbstverständlich nur der Autor verantwortlich. 
Peter Gottfried - 978-3-631-75208-1

Downloaded from PubFactory at 01/11/2019 07:06:43AM

via free access 


\section{Inhaltsverzeichnis}

Einleitung 1

Kapitel I Grundzüge der Umsatzsteuer $\quad 7$

1. Historische Betrachtung $\quad 7$

2. Umsatzsteuertypen 11

2.1. Die Anzahl der steuerpflichtigen Unternehmen 14

2.2. Die materielle Steuerbemessungsgrundlage 17

2.3. Das Erhebungsverfahren 20

2.4. Die Berechnung der Umsatzsteuerschuld 23

2.5. Die unmittelbare Belastungswirkung 27

2.6. Die Besteuerung des Außenhandels 32

3. Das bundesdeutsche Umsatzsteuersystem 34

4. Steuerbefreiungen und Nullsteuersätze 40

Kapitel II Effektive Steuersätze 49

1. Die formale Darstellung der Umsatzsteuer 51

2. Effektive Wertschöpfungsteuersätze 56

3. Der Steuergehalt der Endnachfrage 61

4. Die Berechnung effektiver Steuersätze 65

4.1. Effektive Konsumsteuersätze 66

4.2. Ein numerisches Beispiel , 70

4.3. Außenhandel $\quad 75$

4.4. Investitionen $\quad 80$

5. Die effektiven Steuersätze aus der Datenbasis $\quad 87$

Kapitel III Das Empirische Allgemeine Gleichgewichtsmodell 91

Teil A: Die Modellstruktur $\quad 91$

1. Der repräsentative Haushalt 94

2. Die Unternehmen 100

2.1. Investitionen 100

2.2. Produktion- und Faktorsteuern 102

2.3. Die Faktornachfragefunktionen 109

3. Außenhandel 110

4. Der Staat 113

5. Marktgleichgewichte 115 
Teil B: Die Erstellung einer konsistenten Datenbasis 117

1. Die Input-Output-Tabelle des statistischen Bundesamtes

2. Zentralmatrix und nominelle Umsatzsteuersätze

3. Die Matrix der Primärinputs

4. Endnachfrage und Budgetrestriktionen 140

5. Abschließende Bemerkungen

Teil C: Kalibrierung und Wahl der exogenen Parameter

1. Kalibrierung oder ökonometrischer Ansatz 148

2. Unternehmensbereich 151

3. Haushaltsbereich 153

4. Die Parameterwerte im Einzelnen 156

Kapitel IV Steuersimulationen 161

1. Vorbemerkungen 161

1.1. Der Modellablauf 161

1.2. Budget- und Differentialinzidenz 163

1.3. Lösungsalgorithmus und Eindeutigkeit des Gleichgewichts

1.4. Die ökonomische Beurteilung der Steuerreform $\quad 169$

2. Der Übergang zu Nullsteuersätzen $\quad 172$

2.1. Die Änderung der Struktur der Konsumsteuersätze 173

2.2. Die Änderung der Besteuerung des Außenhandels $\quad 174$

2.3. Die Änderung der Investitionsteuersätze 179

3. Die Erweiterung der effektiven Steuersätze 182

4. Einheitliche und optimale Umsatzsteuersätze 128

5. Sensitivitätsanalyse 193

5.1. Die Außenhandelselastizitäten 193

5.2. Arbeitsangebots- und Substitutionselastizitäten 194

5.3. Das LES-Nachfragesystem 196

6. Abschließende Bemerkungen 199

Anhang 203

$\begin{array}{ll}\text { Literaturverzeichnis } & 218\end{array}$ 


\section{Einleitung}

Wie alle bedeutenden Steuern ist auch die Umsatzsteuer in steter Regelmäßigkeit Gegenstand wirtschaftspolitischer und insbesondere finanzwissenschaftlicher Diskussionen. Während sie zu Beginn dieses Jahrhunderts und selbst noch in den fünfziger Jahren nur im europäischen Raum zur Anwendung kam, ist ihre weltweite Bedeutung in den letzten $40 \mathrm{Jahren}$ explosionsartig angestiegen. Diese sprunghafte Entwicklung wurde von TAIT ([1988], S. 3) mit folgendem blumigen Vergleich beschrieben: „... VAT may be thought of as the Mata Hari of the tax world - many are tempted, many succumb, some tremble on the brink, while others leave only to return, eventually the attraction appears irresistible."

Zum Beispiel stand Japan gleich dreimal - 1950/51, 1980 und 1986/ 87 - kurz vor der Einführung einer Umsatzsteuer. Sie wurde jedoch jedesmal wieder ausgesetzt. Erst 1989 konnte eine Umsatzsteuer europäischen Stils parlamentarisch durchgesetzt werden. In den Vereinigten Staaten bildet die Steuer vor dem Hintergrund des chronisch anwachsenden Budgetdefizits einen nahezu permanenten Diskussionspunkt in wirtschaftspolitischen und finanzwissenschaftlichen Debatten.

Auch in der Bundesrepublik ist ein Anstieg des finanzwissenschaftlichen Interesses an der Umsatzsteuer festzustellen. Neben der anhaltenden Kontroverse, ob indirekte Steuern den direkten vorzuziehen sind, liefert vor allem die EG-Steuerharmonisierungsbestrebung aktuelle Anreize für theoretische Auseinandersetzungen mit bestimmten Aspekten dieser Steuer ${ }^{1}$. Nach der für 1993 geplanten Abschaffung der Grenzkontrollen zwischen den EG-Mitgliedstaaten bereitet hauptsächlich die Konstruktion eines adäquaten Grenzausgleichsverfahrens gewisse Probleme.

Das gegenwärtig praktizierte Bestimmungslandprinzip, d. h. die Freistellung der Exporte und die Erhebung einer Einfuhrumsatzsteuer auf Importe könnte dann nur unter erschwerten Bedingungen beibehalten werden. Ein Übergang zum Ursprungslandprinzip würde dagegen in

${ }^{1}$ Siehe u. a. ANDEL [1986] oder Fehr/Rosenberg/Wiegard [1990]. 
Reinform zu einer starken internationalen Umverteilung des Umsatzsteueraufkommens führen, da hinsichtlich der angestrebten Vereinheitlichung der Umsatzsteuersätze innerhalb der EG-Länder bislang noch keine allzu großen Fortschritte erzielt wurden.

Einer der Streitpunkte im Rahmen der Harmonisierung der Umsatzsteuersätze bildet die Frage, ob umsatzsteuerbefreiten Unternehmen ein Vorsteuer abzugsrecht eingeräumt werden soll oder nicht. Während die Mehrzahl der EG-Länder - so auch die Bundesrepublik Deutschland - Umsatzsteuerbefreiungen vorwiegend mit einem Vorsteuer abzugsverbot kombiniert, gelangt in Portugal, Irland und Großbritannien vorwiegend die alternative Variante zum Einsatz. Aus Gründen, die im weiteren Verlauf dieser Arbeit noch genannt werden, empfahl auch der Rat der Europäischen Gemeinschaften in seiner Sechsten Richtlinie zur EG-Steuerharmonisierung ${ }^{2}$, einen Vorsteuerabzug bei Umsatzsteuerbefreiungen grundsätzlich nicht zu gewähren. Vor allem Großbritannien weigerte sich bislang strikt dieser Empfehlung des EG-Rates nachzukommen. Man befürchtet, daß diese Maßnahme zu einer negativen Entwicklung der Wirtschaft - u. a. Preissteigerungen - beiträgt.

Die Weisung des EG-Rates ist auch in finanzwissenschaftlichen Kreisen nicht unumstritten. Da nichtabzugsberechtigte Unternehmen faktisch auf die Stufe eines Endverbrauchers gestellt werden, haben sie die angefallenen Vorsteuern selbst zu tragen und werden diese über eine entsprechende Preiskalkulation an andere Wirtschaftsubjekte weitergeben. Die hierdurch entstehenden Allokationsverzerrungen sind überaus vielschichtig und nicht ohne Weiteres zu durchschauen - was an den eingangs zitierten Vergleich erinnert. Deshalb wird die Wirkungsweise von Befreiungen bei gleichzeitigem Vorsteuerabzugsverbot zumeist in einzelwirtschaftlicher Betrachtung anhand von Kontendarstellungen erörtert ${ }^{3}$ oder, auf bestimmte Gesichtspunkte beschränkt, die tendenzielle Wirkungsrichtung aufgezeigt ${ }^{4}$. Eine gesamtwirtschaftliche Einschätzung unterbleibt jedoch häufig.

${ }^{2}$ Sechste Richtlinie des Rates vom 17. 5. 1977 zur Harmonisierung der Rechtsvorschriften der Mitgliedstaaten über die Umsatzsteuer, Amtsblatt der Europäischen Gemeinschaften, 20. Jg., 1977.

${ }^{3}$ Siehe bspw. McLure/Ture [1972].

¿ Sie bspw. POHMER [1983]. 
In diesem Sinne schlug C. E. MCLuRE JR. - einer der führenden Umsatzsteuer-Experten in den Vereinigten Staaten - die Formulierung eines Allgemeinen Gleichgewichtsmodells vor, das die Vorleistungsverflechtung der Unternehmen beschreibt und zudem eine detaillierte Abbildung der unterschiedlichen Besteuerungsregelungen erlaubt. Er führt weiterhin aus ([1987a], S. 21): „I am unaware of any study that actually follows this methodology." Dem Aufruf wird hiermit Folge geleistet.

Angesichts der Aufgabenstellung bietet sich die Verwendung eines Empirischen Allgemeinen Gleichgewichtsmodells unmittelbar an. Einerseits gestattet die mikroökonomische Modellstruktur eine präzise Beschreibung der Besteuerungsregelungen auf einzelwirtschaftlicher Ebene, andererseits ermöglicht es die numerische Ausgestaltung des Modells, die Steuer in einem relativ komplexen ökonomischen Umfeld zu untersuchen und dennoch inhaltsreiche Aussagen zu gewinnen.

In nahezu allen bislang existierenden empirischen Modellen, die auch eine Umsatzsteuer berücksichtigen, wird letztere zumeist vereinfacht als Konsumsteuer dargestellt ${ }^{5}$. Diese Vereinfachung erscheint durchaus legitim und sinnvoll, wenn man bedenkt, daß die weltweit am häufigsten praktizierte Umsatzsteuervariante eine Umsatzsteuer vom Konsumtyp ist. Aber gerade die verdeckten Wirkungen von Umsatzsteuerbefreiungen ohne Vorsteuerabzugsrecht werden damit übergangen. Die relative Bedeutung dieser Wirkungen kann beispielsweise daran ermessen werden, daß gemäß der Input-Output-Tabelle für 1984 der Anteil der auf dem Konsum lastenden nichtabzugsfähigen Umsatzsteuerbeträge lediglich rund $63.1 \%$ des gesamtwirtschaftlich erzielten Umsatzsteueraufkommens betrug.

Eine rein deskriptive Methode, um die tatsächliche Wirkung der Umsatzsteuer kenntlich zu machen, wurde von KAY/WARREN [1980] entwickelt. In Anlehnung an die Berechnung der effektiven Schutzwirkung von Zöllen ${ }^{6}$ ermittelten sie effektive Steuersätze aus den gegebenen no-

${ }^{5}$ Siehe bspw. Ballard/Scholz/Shoven [1978] oder KeHoe/NoyoLA/MANRESA/Polo/SANCHO [1988], eine rühmliche Ausnahme hierzu, bildet das INTIMO-Modell Italiens (vgl. BARDAZZI/GRASSINI/LANGOBARDI [1989]).

${ }^{6}$ Siehe bspw. ROSE [1981], S. 441 f.. 
minalen Umsatzsteuersätzen. Allerdings ist nach dieser Methode - wie wir noch sehen werden - die durch die Umsatzsteuerbefreiungen auftretende indirekte Besteuerung nicht erfaßbar. Um auch hier Abhilfe zu schaffen, wird in der vorliegenden Arbeit ein Verfahren zur Berechnung effektiver Steuersätze vorgestellt, welches die verdeckte Besteuerung miteinbezieht.

Die Aussagekraft der neu entwickelten effektiven Steuersätze kann wiederum im Rahmen des Empirischen Allgemeinen Gleichgewichtsmodells überprüft werden. Dabei zeigt sich, daß die eigentliche Umsatzsteuer durch das System der effektiven Steuersätze ersetzt werden kann, ohne hierdurch die Güter- und Faktorallokation zu verändern. Folglich sind die berechneten effektiven Steuersätze in diesem Sinne vollkommen äquivalent zu den ursprünglichen nominalen Umsatzsteuersätzen. Im Gegenzug können erstere dann wiederum verwendet werden, um die mit dem Allgemeinen Gleichgewichtsmodell errechneten Allokationswirkungen verschiedener Umsatzsteuerreformen - insbesondere der Einführung eines allgemeinen Vorsteuerabzugsrechts - besser interpretierbar zu machen.

Aus diesen einführenden Bemerkungen geht auch der Aufbau der Arbeit hervor. Kapitel I dient vor allem der Bereitstellung der notwendigen Hintergrundinformationen, die zu einem besseren Verständnis der vorgegebenen Problematik beitragen. Nach einer kurzen historischen Skizze werden zunächst die verschiedenen Gestaltungsmöglichkeiten einer Umsatzsteuer dargestellt. Mit der Beschreibung der verschiedenen Umsatzsteuertypen wird hauptsächlich das Ziel verfolgt, die einzelnen Techniken der Besteuerung gegeneinander abzugrenzen und deren Wirkungsweise in einzelwirtschaftlicher und gesamtwirtschaftlicher Sichtweise offenzulegen. Damit ist gleichzeitig das Wesen der gegenwärtig in der Bundesrepublik praktizierten Umsatzsteuer im Vergleich zu anderen Erhebungsformen besser erkennbar und auch der Unterschied zwischen Vorsteuerabzugsrecht und Vorsteuer abzugsverbot besser einzuordnen. Im letzten Abschnitt des ersten Kapitels sind schließlich einige Argumente pro und contra Vorsteuerabzugsverbot einander gegenübergestellt.

Da die effektiven Steuersätze ein deskriptives Instrument zur Beurteilung der von der Umsatzsteuer ausgehenden Wirkungen darstellen, 
welches nicht an den Einsatz eines Allgemeinen Gleichgewichtsmodells gebunden ist, wird ihnen eine eigenständige Bedeutung eingeräumt. Die Herleitung der effektiven Steuersätze erfolgt in Kapitel II. Als hinführende Maßnahme werden dabei zuerst die Vorgehensweise von KAY und WARREN sowie zwei Methoden für eine Inzidenzbetrachtung im Rahmen eines vereinfachten Grundmodells mit nur einer Endnachfragekomponente - dem Konsum - dargestellt und auf ihre Eignung für unsere Zwecke geprüft. Diese vorgelagerten Beschreibungen zeigen gleichzeitig den für die Berechnung der effektiven Steuersätze einzuschlagenden Weg. In den nachfolgenden Abschnitten wird schließlich das Grundmodell sukzessive um Investitionen und außenwirtschaftliche Beziehungen erweitert.

Kapitel III erklärt den Aufbau des verwendeten Empirischen Allgemeinen Gleichgewichtsmodells. Das Kapitel ist in drei Teile untergliedert, die sich an den notwendigen Arbeitsgängen orientieren. Teil A enthält die theoretische Modellstruktur und macht auf die spezifische Problematik aufmerksam, die durch die Wahl von konkreten funktionalen Formen zur Beschreibung der Verhaltensfunktionen entsteht. Teil B widmet sich der Konstruktion einer zum Modell konsistenten Datenbasis. Da die Implikationen, die das theoretische Modell beinhaltet, auch von den Daten erfüllt werden müssen, sind Anpassungen des statistischen Ausgangsmaterials unumgänglich. In Teil $\mathrm{C}$ werden schließlich die exogenen Parameter der unterstellten funktionalen Beziehungen mit numerischen Werten ausgestattet. Dieses als Kalibrierung bezeichnete Verfahren unterliegt mit der heftigsten, an Empirischen Allgemeinen Gleichgewichtsmodellen geäußerten Kritik. Wir werden im ersten Abschnitt von Teil $\mathrm{C}$ etwas näher darauf eingehen.

Das abschließende Kapitel IV präsentiert die mit dem Empirischen Gleichgewichtsmodell berechneten Ergebnisse. Auf einige einführende Bemerkungen zum technischen Ablauf dieser Form der Analyse folgt die Simulation der für unsere Zielsetzung zentralen Steuerreformmaßnahme - der generellen Abschaffung des Vorsteuerabzugsverbots. Der Erklärung der ökonomischen Effekte wird hierbei ein besonderer Stellenwert eingeräumt. Gleichzeitig finden die zuvor ermittelten effektiven Steuersätze Verwendung als deskriptives Hilfsmittel. Neben dieser zentralen Steuerreform wird auch untersucht, welche Effizienzwirkungen 
aus der Einführung eines allgemeinen Umsatzsteuersatzes zu erwarten sind und nach welchen Gesichtspunkten das optimale Umsatzsteuersystem in unserer komplexen Modellwelt ausgestaltet sein sollte. Zum Abschluß erfolgt schließlich eine Prüfung, wie robust die errechneten Ergebnisse hinsichtlich einer Variation der zentralen Bestimmungsfaktoren sind. 


\section{Kapitel I}

\section{Grundzüge der Umsatzsteuer}

\section{Historische Betrachtung}

Obwohl die Umsatzsteuer erst zu Beginn bzw. Mitte des 20. Jahrhunderts ihre große internationale Bedeutung erlangte - im europäischen Raum wurde sie 1916 in Deutschland, 1917 in Frankreich und 1919 in Italien eingeführt, danach folgten Belgien (1921), Großbritannien (1940), Schweden und die Schweiz (1941) -, stellt sie keineswegs eine völlig neuartige Besteuerungsform dar. Bereits im 5 . vorchristlichen Jahrhundert war den griechischen Stadtstaaten die Besteuerung der Umsätze bekannt. So erhob beispielsweise Athen neben Kopfsteuern und Zöllen auch eine Grundstücksumsatzsteuer, eine Marktumsatzsteuer, die den Einzelhandel traf, und eine allgemeine Umsatzsteuer, die hauptsächlich den Großhandel belastete. Wann diese Art Steuer erstmals eingesetzt wurde, ist nicht bekannt. GRABOWER, HERTIG und SCHWARZ [1962] schätzten ihre Entstehung in Griechenland auf das 8. bzw. 7. Jahrhundert v. Chr. Noch im hellenistischen Ägypten gehörte die sog. Enkyklion zu den vier Haupteinnahmequellen des Staates, wobei der Name Enkyklion in seiner Grundbedeutung „sich im Kreise bewegen“ bereits auf ihren Charakter als Verkehrsteuer - wie Umsatzsteuern auch heute aufgefaßt werden - hinweist.

Im römischen Imperium wurde die Umsatzbesteuerung über mehrere Jahrhunderte hinweg praktiziert. Nach hellenistischem Vorbild von Augustus zur Deckung der Pensionszahlungen ausgedienter Legionäre eingeführt, bestand sie ursprünglich aus einer einprozentigen Steuer auf Auktionsumsätze (einer im römischen Reich sehr häufig anzutreffenden Marktform) und einer Sklavenumsatzsteuer in Höhe von zwei Prozent. Im Laufe der Zeit wurden zwar Steuersätze und Besteuerungsobjekt verändert - so erweiterte beispielsweise der römische Kaiser Caligula die Steuerpflicht auf die Umsätze von Lebensmitteln -, die Umsatzsteuer selbst blieb jedoch bis ins Ostgotenreich unter Theoderich, hier allerdings auf Marktumsätze bezogen, in Kraft. 
Die dritte, wohl bekannteste und zugleich berüchtigste Frühform der Umsatzsteuer war die spanische alcabala, die alle Umsätze sämtlicher Wirtschaftsstufen einschloß. 1342 zunächst nur periodenweise zur Deckung der Kriegskosten eingeführt, bildete sie später eine nicht unwesentliche permanente Einnahmenquelle mit zeitweise extrem hohen Steuersätzen, welche die spanische Wirtschaft schwer belasteten. So betrug der Steuersatz unter Philipp II. bis zu 15 Prozent. Die Steuer wurde nicht nur in Spanien selbst, sondern auch in den spanischen Provinzen wie zum Beispiel Holland - trotz heftigen Widerstands der Bevölkerung - erhoben. Erst Ferdinand VII schaffte diese Form der Umsatzbesteuerung um $1815 \mathrm{ab}$.

In Zentraleuropa erhoben zwar die Franken, das römische Finanzrecht adaptierend, eine Marktumsatzsteuer. Sie hatte jedoch keine große Bedeutung und wich mit den nach 1150 einsetzenden Autonomiebestrebungen der großen Städte, auf die sukzessive auch die Steuerhoheit überging, der Erhebung von Wege- und Torzöllen. Im 17. Jahrhundert war sie bis auf wenige Ausnahmen (bspw. Schlesien) auf deutschem Reichsgebiet nicht mehr anzutreffen.

Schon seit der Römerzeit traf die Einführung der Umsatzsteuer immer wieder auf den Widerstand der Bevölkerung - im Mittelalter schimpfte man sie indebita exactio oder Ungelt. Umso erstaunlicher erscheint dann die Einführung einer Umsatzsteuer 1863 in Bremen auf Betreiben der dort ansässigen Kaufleute. Sie diente als Ersatz für die Abschaffung einer Akzise, wurde aber bereits 1884 - wiederum auf Betreiben der Kaufleute - außer Kraft gesetzt, nachdem jene eine Verringerung der Wettbewerbsfähigkeit gegenüber anderen großen Handelsstädten feststellen mußten. Die Umsätze gingen zurück, da sich die Transaktionen zur Vermeidung der Steuer auf benachbarte Handelszentren verlagerten.

Zur Vervollständigung der historischen Skizze sei noch erwähnt, daß Frankreich 1292-1642 periodenweise zur Deckung vorübergehender Aufkommensprobleme auf die Besteuerung der Umsätze zurückgriff und einige italienische Städte diese Besteuerungsform angewandten. Während der Sezessionskriege führte auch Nordamerika 1864 eine allgemeine Umsatzsteuer ein, die jede Wirtschaftsstufe - insbesondere auch die Privatentnahme seitens der Unternehmer - mit Steuersätzen von 
3-6 v. H. traf. Hier waren es überraschenderweise ebenfalls die Unternehmer, die bei der Notwendigkeit staatlicher Mehreinnahmen für eine Erhöhung der Umsatzsteuer eintraten. Sie waren in dieser Zeit offensichtlich in der Lage, Preise durchzusetzen, die die Steuererhöhung überkompensierte. Nach Kriegsende wurde die Steuer relativ schnell durch sukzessive Steuerbefreiungen nach einzelnen Güterarten wieder abgeschafft.

Der erste Vorläufer der gegenwärtig in der Bundesrepublik praktizierten modernen Umsatzsteuer ist wohl in der 1916 verabschiedeten Stempelsteuer auf Warenumsätze zu sehen. Wie so oft, führte die durch einen Krieg - den Ersten Weltkrieg - bedingte finanzielle Zwangslage zur Erhebung einer Abgabe in Höhe von $1.5 \mathrm{v}$. H. auf die Lieferungen von Waren innerhalb des inländischen Gewerbes. Schon 1918 wurde die Steuer auf die in selbständiger Tätigkeit ausgeführten Leistungen bei einem Steuersatz von nun 0.5 v. H. erweitert. Nur unselbständige Lohnempfänger und freiberuflich Tätige blieben von der Steuer verschont - letztere allerdings nur für ein Jahr. Mit dem Gesetz vom 24. 12. 1919 wurden auch freie Berufe der Steuerpflicht unterzogen und der Steuersatz auf 1.5 v. H. angehoben.

Diese Allphasen-Bruttoumsatzsteuer wurde im Laufe ihres 50-jährigen Bestehens mehrmals geändert. Zu den wichtigsten Veränderungen zählt die 1932 verabschiedete Einführung eines Belastungsausgleichs für den grenzüberschreitenden Warenverkehr: der Import von Gegenständen wurde besteuert und im Gegenzug auf Exporte eine Ausfuhrvergütung gewährt. Eine zweite bedeutsame Gesetzesänderung erfuhr sie 1934. Der Großhandel unterlag fortan einem ermäßigten Steuersatz, wobei man eine umfangreiche Liste von Großhandelsumsätzen notwendiger Rohstoffe und Halberzeugnisse gänzlich von der Steuer befreite. Darüber hinaus wurde die Unselbständigkeit von in Organschaften eingegliederten Unternehmen im Umsatzsteuerrecht gesetzlich verankert.

Die jüngere der beiden angesprochenen Gesetzesänderungen basierte wohl schon auf der Notwendigkeit, die kumulative Wirkung der Steuer und die damit verbundenen negativen wirtschaftspolitischen Effekte abzuschwächen. Sie förderte unter anderem die vertikale Unternehmenskonzentration und belastete die Waren und Dienstleistungen in gewis- 
sem Maße relativ willkürlich. Dennoch war dieses Umsatzsteuersystem bis Ende 1967 in Kraft. Zwei Ereignisse verschärften jedoch die Dringlichkeit einer grundlegenden Reform. Zum einen konstatierte ein Urteil des Bundesverfassungsgerichts 1966 den Mangel an Wettbewerbsneutralität, soweit es sich um einstufige und mehrstufige Unternehmen handelte ${ }^{7}$, zum anderen wurden vom Ministerrat der EWG die erste und zweite Richtlinie zur Harmonisierung der Umsatzsteuer erlassen ${ }^{8}$. Danach sollten die Mitgliedstaaten bis spätestens 1. 1. 1970 ein der Richtlinie entsprechendes Umsatzsteuersystem einführen. Als Vorbild diente das in Frankreich bereits seit 1954/55 praktizierte Nettosystem ${ }^{9}$. Die Verabschiedung des neuen „wettbewerbsneutralen“ Umsatzsteuergesetzes erfolgte am 29.5. 1967. Es trat am 1. 1. 1968 - in Anlehnung an die französische Bezeichnung Taxe sur la Valeur Ajoutée - unter dem Namen Mehrwertsteuergesetz in Kraft.

Die letzte größere Gesetzesänderung wurde 1980 wiederum vor dem Hintergrund einer Umsatzsteuer-Richtlinie der EWG ${ }^{10}$ vollzogen. Man beschloß, die Finanzbeiträge der Mitgliedstaaten durch Einnahmen aus dem gemeinsamen Zolltarif, den Agrarabschöpfungen und der Mehrwertsteuer in Form von einem Prozent einer für alle Länder vereinheitlichten Bemessungsgrundlage zu ersetzen. Die Vereinheitlichung betraf hauptsächlich die bislang national unterschiedlichen Bemessungsgrundlagen für den Arbeitnehmer- und Gesellschafterverbrauch. Daneben wurde auch der Ort der Leistung, der letztendlich den Ausschlag dafür gibt, in wessen Hoheitsbereich die zu entrichtende Umsatzsteuerschuld

${ }^{7}$ Bundessteuerblatt III 1967 S.7.

${ }^{8}$ Erste Richtlinie des Rates vom 11. 4. 1967 zur Harmonisierung der Rechtsvorschriften der Mitgliedstaaten über die Umsatzsteuer, Amtsblatt der Europäischen Gemeinschaften 10. Jg., 1967, S. 1301-1303.

Zweite Richtlinie des Rates vom 11. 4. 1967 zur Harmonisierung der Rechtsvorschriften der Mitgliedstaaten über die Umsatzsteuer - Struktur und Anwendungsmodalitäten des gemeinsamen Mehrwertsteuersystems, Amtsblatt der Europäischen Gemeinschaften 10. Jg., 1967, S. 1303-1312.

${ }^{9}$ Vgl. hierzu u. a. LIST [1983] S. 15.

${ }^{10}$ Sechste Richtlinie des Rates vom 17. 5. 1977 zur Harmonisierung der Rechtsvorschriften der Mitgliedstaaten über die Umsatzsteuern - Gemeinsames Mehrwertsteuersystem: einheitliche steuerpflichtige Bemessungsgrundlage, Amtsblatt der Europäischen Gemeinschaften, 20. Jg., 1977, Nr. L 145, S. 1-40. 
fällt, neu definiert. Diese neue Auslegung des Ortes der Leistung diente zur Lösung von Kompetenzstreitigkeiten, die beispielsweise dann auftraten, wenn mit der grenzüberschreitenden Lieferung eine Montage verbunden war. Im gleichen Zusammenhang wurde mit der Einführung des sog. Abzugsverfahrens ${ }^{11}$ darüber hinaus sichergestellt, daß ein Unternehmer, der seinen Sitz nicht im Erhebungsgebiet hat, dort aber Leistungen erbringt, die eingenommene Steuer auch tatsächlich abführt ${ }^{12}$.

Das Umsatzsteuersystem der BRD ging somit aus einer Reihe von Reformen hervor, deren Kenntnis zum besseren Verständnis des aktuellen Systems beitragen. In jüngerer Zeit standen die Reformen im Zeichen der Umsatzsteuer-Harmonisierung in den EG-Mitgliedstaaten, die - abgesehen von den national angewandten Steuersätzen - auch weitgehend verwirklicht werden konnte. Bevor wir uns jedoch der konkreten Ausgestaltung der Umsatzsteuer in der Bundesrepublik zuwenden, werden kurz die verschiedenen Umsatzsteuertypen angesprochen.

\section{Umsatzsteuertypen}

Die möglichen Erscheinungsformen der Umsatzsteuer sind außerordentlich vielfältig. Man denke hierbei weniger an die Möglichkeit, die Steuersätze unterschiedlich zu differenzieren, sondern eher an allgemeine konzeptionelle Gestaltungsmöglichkeiten des Steuerzugriffs, der Bemessungsgrundlage usw ${ }^{13}$.

Hinsichtlich der Besteuerungstechnik werden Umsatzsteuertypen in erster Linie nach

- der Anzahl der steuerpflichtigen Unternehmen,

- der Ausgestaltung der materiellen Bemessungsgrundlage,

- dem Steuererhebungsverfahren und

- der Berechnung der Umsatzsteuerschuld

$11 \S 18$ Abs. 8 Umsatzsteuergesetz (UStG).

12 Näheres siehe bspw. GoERGEN [1985], S. $16 \mathrm{ff}$.

${ }^{13}$ Da der Umsatzbegriff in der Wirtschaftswissenschaftlichen Literatur nicht einheitlich verwendet wird, sei an dieser Stelle auf die hier verwendete steuerrechtliche Definition als „die Lieferungen und Leistungen, die ein Unternehmer im Erhebungsgebiet gegen Entgelt im Rahmen seines Unternehmens ausführt“ ( $§ 1$ Abs. 1 UStG) hingewiesen. 
charakterisiert. Nicht alle dieser Kriterien sind auf jeden Umsatzsteuertyp anwendbar, was die in Figur 1 dargestellte Systematik zeigt. So unterscheidet man zunächst nach der Anzahl der steuerpflichtigen Unternehmen in Einphasen-, Mehrphasen- und Allphasensteuern, wobei letztere wiederum nach der Gestaltung der materiellen Bemessungsgrundlage in Brutto- und Nettoumsatzsteuern getrennt werden. Nettosysteme klassifiziert man ihrerseits nach dem angewendeten Erhebungsverfahren usw. In den anschließenden vier Teilabschnitten werden die nach dieser Systematik unterscheidbaren Umsatzsteuertypen bzw. Besteuerungstechniken etwas näher erläutert, wobei auf die wesentlichen Vor- oder Nachteile, sowie die praktische Relevanz der verschiedenen Formen hingewiesen wird.

Angesichts einer derartigen Vielzahl von Umsatzsteuertypen kann es nicht verwundern, daß auch die Zuordnung der Umsatzsteuer zu einer bestimmten Steuerart Schwierigkeiten bereitet. Während PoPITZ, der Schöpfer der deutschen Umsatzsteuer, sie in der ersten Auflage des Handbuchs der Finanzwissenschaft 1927 noch unter dem Titel "Allgemeine Verbrauchsteuer" behandelte und auch CNOSSEN in seiner umfassenden internationalen Studie die Umsatzsteuer zu den "Excise Systems" zählte, wird sie heute in der Regel zu den Verkehrsteuern gerechnet ${ }^{14}$. Beide Einordnungen haben ihre Berechtigung, sie beziehen sich lediglich auf verschiedene steuerliche Gliederungsgesichtspunkte. Allerdings sind sie von der aktuellen Gestalt der Steuer abhängig und nicht generell für alle Typen passend.

Die Zuordnung zu den Verkehrsteuern zielt eher auf den gesetzestechnischen Ansatzpunkt - den Austausch realer oder monetärer Leistungen als Besteuerungsgrund - ab. „Die Verkehrsbesteuerung nimmt den Verkehrsvorgang selbst zum Anlaß der Steuererhebung." (Rose [1987], S. 19). Für eine bestimmte Form der Nettoumsatzsteuer, genauer gesagt eine nach der additiven Methode auf die Summe der Wertschöpfungskomponenten der Unternehmen erhobene, ist der Verkehrsvorgang jedoch sehr stark in den Hintergrund gerückt. Besteuert wird vielmehr der Unternehmensertrag und folglich müßte diese Va-

${ }^{14}$ Stellvertretend sei hier auf POHMER [1980], Rose [1987] und BEA [1980] verwiesen. Für eine Auseinandersetzung mit dieser Zuordnungsproblematik siehe auch PHILIPOWSKI [1985] S. $183 \mathrm{ff}$. 
Figur 1: Übersicht der verschiedenen Umsatzsteuersysteme

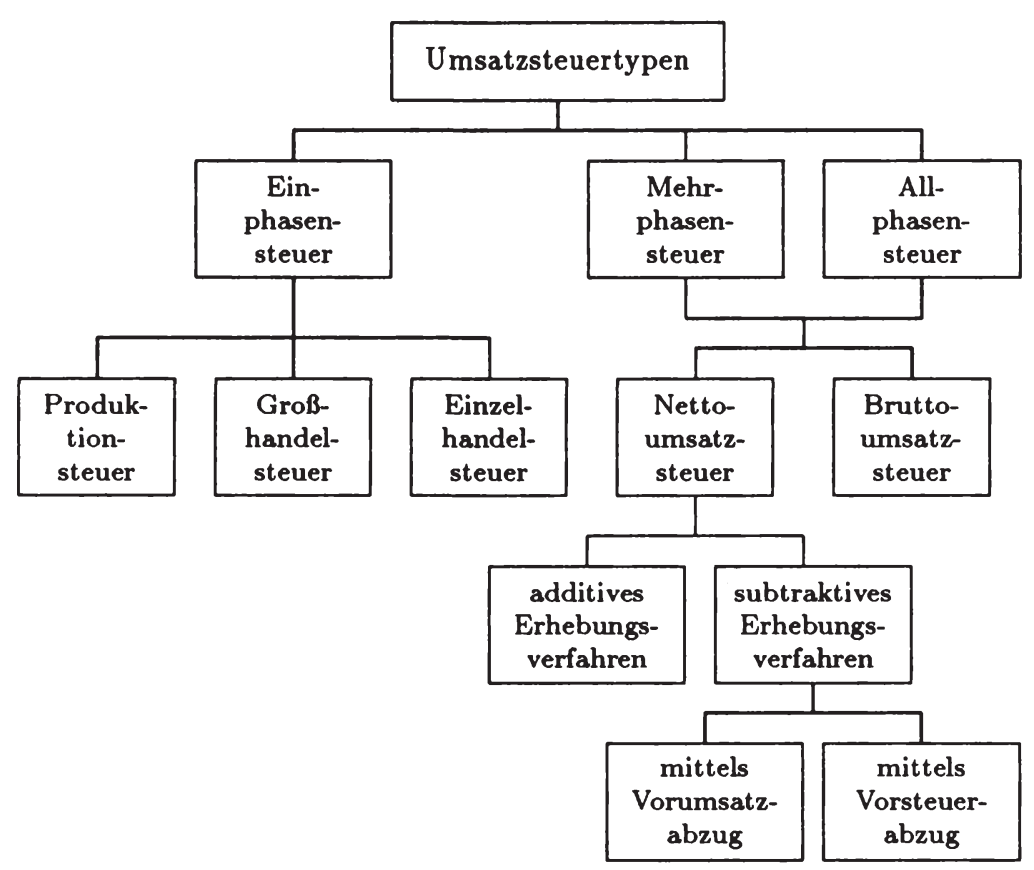

Quelle: DzIADKowsKi [1986], S. 5

riante zu den Ertragsteuern gerechnet werden. Obwohl sie sich aus der zugrundegelegten Systematik zwangsläufig ergibt, erscheint selbst der Name Umsatzsteuer nicht mehr angemessen. Der im englischen Sprachraum verwendete Oberbegriff Value-Added Tax (Wertschöpfungsteuer) wäre hier wohl treffender.

Die Einordnung zu den Verbrauchsteuern stellt hingegen auf die vom Gesetzgeber intendierte Belastungswirkung - die indirekte Besteuerung des privaten Konsums - ab. In der Tat sind die meisten praktizierten Umsatzsteuertypen so konzipiert, daß letztendlich der private Endverbrauch von der Steuer getroffen wird. Durch eine geeignete Ausge- 
staltung der Bemessungsgrundlage bzw. durch Regulierung der Anzahl der steuerpflichtigen Unternehmen ist es jedoch möglich, nicht nur den Konsum, sondern auch andere volkswirtschaftliche Aggregatsgrößen wie bspw. das Bruttoinlands- oder Bruttosozialprodukt mit der Steuer zu belasten. Der vierte Unterabschnitt ist deshalb der Charakterisierung von Steuertypen nach der umittelbaren Belastungswirkung gewidmet. Wir wollen von einer unmittelbaren Belastungswirkung sprechen, da es sich nicht um eine Inzidenzbetrachtung handelt, wie sie u. a. von PECHMAN und OKNer [1974] durchgeführt wurde, sondern lediglich um die Darstellung, wer bzw. welche gesamtwirtschaftliche Größe unter Einbeziehung der gesetzlich geregelten Steueranrechnungsverfahren im Endeffekt besteuert werden soll.

Der letzte Teilabschnitt erläutert schließlich die verschiedenen Alternativen der steuerlichen Behandlung des grenzüberschreitenden Güterverkehrs. Die Beschreibung der Außenhandelsbesteuerung wird der Klassifikation von Umsatzsteuertypen nach der unmittelbaren Belastungswirkung deshalb nachgelagert, weil sie ebenso wie diese nicht zwingend in die in Figur 1 dargestellte Gliederungssystematik einzuordnen ist, aber eng mit der intendierten Belastungswirkung zusammenhängt.

\subsection{Die Anzahl der steuerpflichtigen Unternehmen}

Die Gliederung von Umsatzsteuertypen nach der Anzahl der steuerpflichtigen Unternehmen nimmt Bezug auf die in Figur 2 skizzierten funktionellen Wirtschaftsstufen (Phasen), welche die Güter von der Herstellung bis hin zur letzten Verwendung durchlaufen. Erstreckt sich die Steuerpflicht auf die Umsätze aller Unternehmen jeder Fertigungsund Handelsstufe, spricht man von einer Allphasensteuer. Bei Einphasen- und Mehrphasensteuern hingegen sollen die Güter - wie der Name bereits vermuten läßt - nur auf einer einzigen bzw. einzelnen Wirtschaftstufen besteuert werden. Da Mehrphasensteuern in der steuertechnischen Ausgestaltung den Allphasensteuern sehr ähnlich sind und darüber hinaus in der Praxis nur eine untergeordnete Rolle spielen, stehen in den folgenden Ausführungen die verschiedenen Grundtypen von Einphasen- und Allphasensteuern im Vordergrund. 
Figur 2: Funktionelle Wirtschaftsstufen

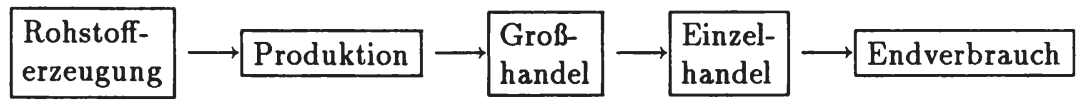

Der wesentliche Vorteil der Einphasensteuer besteht in dem auf eine vergleichsweise geringe Anzahl von Umsätzen beschränkten Steuerzugriff und damit einem niedrigeren Erhebungs- und Verwaltungsaufwand als bei einer Allphasensteuer. Dieser Vorteil muß allerdings etwas relativiert werden, denn die Trennung von steuerpflichtigen und nicht steuerpflichtigen Umsätzen kann mitunter erheblichen Aufwand erfordern. Sie gestaltet sich umso schwieriger, je höher der Verflechtungsgrad einer Volkswirtschaft ist, d. h. je häufiger Güter auf ihrem Weg zum Endabnehmer dieselbe Wirtschaftsstufe durchlaufen. Soll die Abgabe beispielsweise nur die (letzte) Produktionsstufe treffen - man spricht in diesem Fall von einer Produktionsteuer - sind diejenigen Güter, die als Vorleistungen in anderen Unternehmen eingesetzt werden, von der Steuer auszuschließen, um Mehrfachbesteuerungen des Endprodukts zu vermeiden. Den Gütern selbst ist ihr Verwendungszweck jedoch nicht immer unmittelbar anzusehen. Aus diesem Grund eignen sich allgemeine Produktionsteuern noch am ehesten für Volkswirtschaften mit relativ überschaubarer Produktionsstruktur und sind deshalb in der Praxis hauptsächlich in Entwicklungsländern ${ }^{15}$ zu finden.

Die Abgrenzung von steuerbaren und nicht steuerbaren Umsätzen erfolgt dabei in der Regel über die Registrierung der entsprechenden Unternehmen (ggf. mit Optionsmöglichkeit für bestimmte Wirtschaftsbereiche), die, damit verbunden, zu speziellen Aufzeichnungspflichten herangezogen werden. Die Steuer wird dann nur bei Umsätzen registrierter Unternehmen an nicht registrierte erhoben, während die Leistungen der registrierten untereinander oder von nicht registrierten an registrierte nicht steuerpflichtig sind ${ }^{16}$.

${ }^{15}$ Nach CNOSSEN [1977] (S. 133 ff.) sind das Bangladesh, Ghana, Guyana, Kenia, Malaysia, Pakistan, Tansania und Uganda. Die einzige Ausnahme als entwickelte Volkswirtschaft bildet Kanada (vgl. auch DUE [1988], S. 96 ff.).

${ }^{16}$ Eine ausführlichere Beschreibung des Registrierungsverfahrens befindet sich u. a. in POHMER [1977], S. 675 ff. 
Ein analoges Argument trifft für die rein auf Großhandelsebene ansetzende Grossistensteuer zu. Beziehen Unternehmen ihre Vorleistungen über Großhandelsfirmen und liefern ihrerseits die Endprodukte zunächst an den Großhandel, steht auch hier dem - aufgrund einer begrenzten Anzahl von Steuersubjekten - geringeren Verwaltungsaufwand ein zusätzlicher Aufwand für Maßnahmen zur Vermeidung von Mehrfachbesteuerungen gegenüber. Hinzu kommen Erfassungsprobleme in denjenigen Wirtschaftsbereichen, die den Großhandel teilweise umgehen und die Güter direkt dem Einzelhandel oder Endverbraucher zuführen.

Man unterzieht deshalb in der Praxis häufig Produktions- und Großhandelsunternehmen gemeinsam der Registrierungspflicht, wodurch im Grunde die Bezüge des Einzelhandels besteuert werden. Zur Realisierung eines allgemeinen Belastungskonzepts müßten bei dieser Einzelhandel-Vor-Umsatzsteuer konsequenterweise auch die Dienstleistungsunternehmen, deren Güter bzw. Leistungen zum Großteil direkt in den privaten Verbrauch gelangen, registrierungspflichtig sein. In allen Ländern, die eine Einzelhandel-Vor-Umsatzsteuer praktizieren ${ }^{17}$, sind die Umsätze des Dienstleistungsbereichs jedoch nicht steuerbar, so daß dieses System eine reine Warenumsatzsteuer darstellt.

Alternativ zum Registrierungsverfahren besteht die Möglichkeit, alle Güterströme zwischen Unternehmen grundsätzlich von der Steuerpflicht auszuschließen. Damit unterliegen letztendlich nur die Lieferungen an Nichtunternehmer - in der Regel der private Verbrauch - der Steuer. Diese Einzelhandelsteuer hat den Vorteil, daß die Trennung von steuerbaren und nicht steuerbaren Umsätzen - abgesehen von wenigen Sonderfällen wie bspw. der Behandlung von Wiederveräußerungen langlebiger Konsumgüter - keine großen Schwierigkeiten bereitet. Sie bedingt auf der anderen Seite einen vergleichsweise höheren Verwaltungsaufwand als andere Einphasensteuern, da die Anzahl der Steuersubjekte nicht so stark eingeschränkt werden kann und erlaubt gegenüber dem Registrierungsverfahren weniger Kontrollmöglichkeiten. Darüber hinaus bietet dieser Steuertyp (in der so beschriebenen Reinform) keine

${ }^{17}$ Hierzu zählen Australien, Finnland und die Schweiz. Das von CNOSSEN [1977] (S. $133 \mathrm{ff}$.) weiterhin angeführte Neuseeland stellte 1986 auf eine Netto-Allphasensteuer um (siehe TAIT [1988], S. 12) 
Gestaltungsspielräume, die Belastungskonzeption - die indirekte Besteuerung der privaten Konsumausgaben - ist bereits vorgegeben. $\mathrm{Zu}$ erwähnen bleibt noch, daß auch hier von keinem diese Steuer praktizierenden Land ${ }^{18}$ das Prinzip einer allgemeinen Besteuerung konsequent verfolgt wird; wiederum sind die Umsätze des Dienstleistungsbereichs nicht steuerpflichtig.

\subsection{Die materielle Steuerbemessungsgrundlage}

Während bei Einphasensteuern der Umsatz die Steuerbemessungsgrundlage bildet, sind für Mehr- und Allphasensteuern zwei Typen zu unterscheiden: Bei einer Bruttoumsatzsteuer wird auf jeder (steuerpflichtigen) Fertigungs- und Handelsstufe der Umsatz besteuert, ohne die auf Vorleistungen bereits gezahlte Steuer zu berücksichtigen; bei einer Nettoumsatzsteuer hingegen vermindern die Steuerzahlungen auf Vorleistungen die tatsächliche Steuerschuld in einer noch zu beschreibenden Form. Zwar bildet nach wie vor der Umsatz die nominale Steuerbemessungsgrundlage; durch die Anrechenbarkeit gezahlter Steuern reduziert sich die „materielle“ Bemessungsgrundlage jedoch auf die Differenz zwischen Umsatz und Vorumsatz. Wie aus Figur 1 bereits hervorgeht - und noch näher zu erläutern sein wird - umfaßt der Typus der Nettoumsatzsteuer seinerseits alternative Steuerberechnungsverfahren, deren wesentlicher Unterschied gerade in der Behandlung der Steuern auf Vorumsätze besteht. Der Begriff der materiellen Steuerbemessungsgrundlage ist deshalb so zu verstehen, daß alle Verfahren gemeinsam die Absicht verfolgen, nur die Differenz zwischen Umsatz und Vorumsatz zu besteuern. Inwieweit diese Intention systematisch realisisert werden kann, ist ebenfalls an späterer Stelle zu prüfen.

Als Vorteil von Allphasenbruttoumsatzsteuern werden meist Argumente wie Ergiebigkeit, Unmerklichkeit und einfache steuertechnische Ausgestaltung ins Felde geführt ${ }^{19}$. Keines dieser Argumente besitzt

${ }^{18}$ Barbados, Island, den kanadischen Provinzen (CNOSSEN [1977], S. $133 \mathrm{ff}$.) und in 45 Einzelstaaten der USA (DUE/MIKESELL [1984], S. 7 f.). In den USA wird sie weiterhin in 27 Staaten auch als lokale Gemeindeabgabe verwendet (DUE/MIKESELL [1984], S. 268 ff.).

${ }^{19}$ Siehe bspw. POHMER [1977] S. 673, oder LIST [1983] S. 13. 
jedoch Allgemeingültigkeit oder ist als spezifischer Vorteil des Bruttoprinzips anzusehen. Denn im Grunde erzielen alle Steuern, die Verbrauch oder Einkommen allgemein belasten - so auch die Nettoumsatzsteuer -, ein relativ hohes Steueraufkommen.

Figur 3: Steueraufkommen einer Allphasenbruttoumsatzsteuer

\begin{tabular}{|c|c|c|c|c|c|c|c|c|c|}
\hline \multicolumn{2}{|c|}{ A } & \multirow[b]{2}{*}{4} & \multicolumn{2}{|c|}{ B } & \multirow[b]{2}{*}{6.16} & \multicolumn{2}{|c|}{$\mathrm{C}$} & \multirow[b]{2}{*}{8.41} & \multirow{3}{*}{218.57} \\
\hline 70 & 100 & & 104 & 154 & & 160.16 & 210.16 & & \\
\hline 30 & & & 50 & & & 50 & & & \\
\hline 100 & 100 & & 154 & 154 & & 210.16 & 210.16 & & \\
\hline
\end{tabular}

Das Argument der Unmerklichkeit der Steuer zielt schon eher auf eine bestimmte Eigenheit des Bruttoprinzips ab: Es wird bewußt „eine Steuer von der Steuer" erhoben. Ein einfaches Zahlenbeispiel soll dies verdeutlichen: Figur 3 zeigt die stilisierten Ertragskonten dreier Unternehmen A, B und C, die in einer vertikalen Produktionsbeziehung zueinander stehen. Der Output von Unternehmen A wird von B als Input verwendet, der Output von Unternehmen B wiederum als Vorleistung von C. Zur Vereinfachung der Darstellung sind auf der AufwandSeite nur Vorleistungen und Wertschöpfung, auf der Ertrag-Seite nur der Netto-Umsatz aufgelistet. Bei jeder Markttransaktion sind die Umsätze mit einem Steuersatz von angenommen $4 \% \mathrm{zu}$ versteuern. Zum besseren Verständnis werden außerdem die Umsatzsteuerbeträge nicht in den Konten mitverbucht, sondern als „externer Vorgang“ oberhalb der die Markttransaktionen symbolisierenden Pfeile angegeben. Tatsächlich müßte bspw. der Steuerbetrag über dem ersten Pfeil auf beiden Seiten des Ertragskontos von A hinzuaddiert werden; auf der Aufwand-Seite als zu entrichtende Umsatzsteuerschuld, auf der ErtragSeite zusammen mit dem Netto-Umsatz.

Das in diesem Beispiel erzielte Steueraufkommen beläuft sich - als Summe der einzelnen Steuerbeträge - auf (gerundet) 18.57 DM. Um das gleiche Aufkommen mit einer Einzelhandelsteuer zu erzielen, müßte der Steuersatz immerhin rund $9.3 \%$ betragen. Verbindet man mit der Höhe der Steuersätze eine gewisse Signalwirkung auf die Akzeptanz der Steuer bei den Betroffenen (in letzter Instanz der Endverbraucher), so 
scheint die Allphasensteuer tatsächlich „unmerklicher" zu wirken als andere Umsatzsteuertypen. Die Unmerklichkeit kann jedoch bestenfalls in der Einführungsphase der Steuer ernsthaft angeführt werden. Letztendlich ruft sie (cet. par.) die gleichen Preiserhöhungen hervor wie die erwähnte Alternative.

Der Vorteil eines im Vergleich zu anderen Varianten der Umsatzsteuer einfacheren Besteuerungsverfahrens ist wohl nicht zu bestreiten. Im Gegensatz zum Nettoprinzip sind keinerlei Aufzeichnungen über geleistete Vorumsätze und mehr oder weniger aufwendige Anrechnungsverfahren notwendig. Aber auch dieser Vorteil ist nur bei einem Steuersystem mit einheitlichem Steuersatz gegeben. Eine nach verschiedenen Güterarten differenzierte Besteuerung kann (kontrolliert) nur mit erheblichem Mehraufwand und selbst dann nicht zufriedenstellend erfolgen. Darüber hinaus kann trotz eines einheitlichen Steuersatzes für alle Güter die Steuerbelastung der Endprodukte unterschiedlich hoch sein. Existieren mehrere Produktionswege für die Erzeugung eines bestimmten Gutes, ist die Steuerbelastung davon abhängig, wieviele Unternehmen das Gut bis zur letzten Verwendung durchläuft. Hier macht eine intensive Produktionsverflechtung die Abschätzung der endgültigen Belastung der einzelnen Güter mit der Steuer überaus schwierig, von einer gezielten Steuerung dieser Belastung ganz zu schweigen. Mehr noch, da die Steuer nur an Markttransaktionen ansetzt, fördert sie unabhängig von der Struktur der Steuersätze die vertikale Unternehmenskonzentration. Schließen sich in unserem Zahlenbeispiel die Unternehmen A,B und $C$ zu einem einzigen zusammen, bleibt nur ein Steuerzugriff übrig, das Steueraufkommen sinkt auf 8 DM. Als der allgemeine Steuersatz, zu dem die alte Brutto-Allphasensteuer in der Bundesrepublik erhoben wurde, tatsächlich 4 Prozent betrug, trat dieser wettbewerbsverzerrende Effekt zugunsten von vertikal zusammengeschlossenen Unternehmensgruppen besonders deutlich zu Tage.

Aus demselben Grund ist auch ein exakter Grenzausgleich für international gehandelte Güter unmöglich. Sollen beispielsweise Exporte von der Umsatzsteuer freigestellt werden, müßte die tatsächliche Umsatzsteuerbelastung der exportierten Güter bekannt sein. Durch die Abhängigkeit der steuerlichen Belastung vom Produktionsweg entstehen mit jeder pauschalen Regelung zwangsläufig steuerliche Privilegie- 
rungen und Diskriminierungen unabhängig von der Leistungsfähigkeit der betreffenden Unternehmen.

Diese negativen Effekte der Brutto-Allphasensteuer - mitunter auch Kaskaden- oder Lawinensteuer genannt - werden bei der Nettoallphasensteuer vermieden. Soll nur die Differenz zwischen Umsatz und Vorumsatz eines Unternehmens besteuert werden, so muß jedoch ein ausgebautes Buchführungssystem, anhand dessen die Steuer erhoben und auch kontrolliert werden kann, vorliegen. In Volkswirtschaften ohne ausgeprägte Buchführung ist ein Nettosystem nur schwer realisierbar, was wohl auch der Grund dafür ist, daß Brutto-Allphasensteuern immer noch in unterentwickelten Volkswirtschaften anzutreffen sind ${ }^{20}$.

\subsection{Das Erhebungsverfahren}

Die Idee des Nettoprinzips ist relativ simpel: Eine Mehrfachbesteuerung der Güter unterbleibt, wenn auf jeder Stufe nur der den Gütern hinzugefügte Wert - der Mehrwert - besteuert wird. In diesem Sinne ist auch der häufig zur Nettoumsatzsteuer synonym verwendete Begriff Mehrwertsteuer zu verstehen. Da der Mehrwert im Grunde aus der Wertschöpfung eines Unternehmens resultiert, wird dieser Umsatzsteuertyp im englischen Sprachraum mit Value-Added Tax - also Wertschöpfungsteuer - bezeichnet.

Figur 4: Ertragskonto eines Unternehmens

\begin{tabular}{lc|lc} 
Aufwand & \multicolumn{2}{c}{ Ertrag } \\
\hline Vorleistungen & $V$ & Umsatz & $U$ \\
Abschreibungen & $D$ & Vorratsveränderungen & $V V$ \\
Löhne und Gehälter & $L G$ & selbsterstellte & \\
Steuer - Subventionen & $T^{\text {ind }}$ & Anlagen & $S A$ \\
Nettobetriebsüberschuß & $N B U$ & & \\
\hline Bruttoproduktionswert & $B P W$ & Bruttoproduktionswert & $B P W$
\end{tabular}

${ }^{20}$ Hierzu zählen vor allem afrikanische und asiatische Staaten (CNOSSEN [1977], S. $133 \mathrm{ff}$.). 
Unter diesem Gesichtspunkt legt die nochmalige Betrachtung von Figur 3 nahe, daß Nettosysteme grundsätzlich die Wertschöpfung besteuern, wobei die Steuer selbst entweder direkt über eine Addition der Wertschöpfungskomponenten oder indirekt über die Subtraktion der Vorleistungen von den Umsätzen erhoben werden kann. Diese einfache Kongruenz ist jedoch nur auf einem sehr hohen Abstraktionsniveau gegeben und soll anhand des in Figur 4 dargestellten detaillierteren Produktionskontos etwas relativiert werden.

Beide Kontenseiten ergeben den Bruttoproduktionswert. Eine einfache Umformung zeigt jedoch, daß die Besteuerung der Bruttowertschöpfung zu Marktpreisen $\left(D+L G+T^{i n d}+N B U\right)$ nach der direkten oder additiven Methode nicht nur die Differenz zwischen Umsatz und Vorleistungen des Unternehmens $(U-V)$, sondern ebenso die Vorratsveränderungen $(V V)$ sowie die aus eigenen Produktionsmitteln erstellten langlebigen Wirtschaftsgüter $(S A)$ belastet:

$$
\begin{aligned}
\tau\left(D+L G+T^{i n d}+N B U\right) & =\tau(B P W-V) \\
& =\tau(U-V+V V+S A) .
\end{aligned}
$$

Der Unterschied in der Belastungswirkung beider Verfahren leuchtet auch unmittelbar ein. Weder mit der "Produktion auf Halde“ noch mit der Errichtung selbsterstellter Anlagen sind direkte Marktvorgänge verbunden, an denen das subtraktive Erhebungsverfahren ansetzen könnte. Man müßte bei letzterem die materielle Bemessungsgrundlage um diese beiden Posten erweitern (oder umgekehrt, beim additiven Verfahren kürzen), um theoretisch gleiche Belastungswirkungen zu erzielen. Praktisch gesehen bedeutet dies jedoch einen erheblich größeren Verwaltungsaufwand.

Die beim additiven Verfahren angewandte direkte Besteuerung der Wertschöpfung knüpft an Einkommensgrößen - genauer gesagt, Größen der Einkommensentstehung - an. Hat man bei der Ausgestaltung der Steuer beispielsweise die indirekte Besteuerung des (Volks-) Einkommens im Auge, so ist die additive Methode, auf die Nettowertschöpfung zu Faktorkosten $(L G+N B U)$ gerichtet, die natürlichere Vorgehensweise, wobei dann die Kapitalverflechtung der Unternehmen durch eine entsprechende Abgrenzung der Bemessungsgrundlage (Hinzurech- 
nung bzw. Kürzung von Zinserträgen, Mieten, Pachten und dgl.) zu berücksichtigen ist. Folglich ist im Vergleich zu den bisher behandelten Umsatzsteuertypen ein grundlegend anderes Verständnis mit diesem Typ verbunden. Zwar führt die gewählte Systematik der Umsatzsteuertypen zwangsläufig zum additiven Erhebungsverfahren, aber die Betonung liegt bei letzterem nicht mehr auf einer güterorientierten (Verbrauchs-) Besteuerung, sondern eher auf einer Besteuerung des Unternehmenserfolgs. Der Oberbegriff Verkehrsteuer (selbst der Name Umsatzsteuer) ist hier viel schwieriger zu begründen als die Bezeichnung Ertragsteuer. Die Verwandtschaft zur Gewerbesteuer älteren Stils (mit ihren Bestandteilen Gewerbeertrag-, Gewerbekapital- und Lohnsummensteuer) muß wohl nicht weiter betont werden ${ }^{21}$. In diesem Zusammenhang sei noch angemerkt, daß eine Reihe von Finanzwissenschaftlern - insbesondere auch der Wissenschaftliche Beirat beim Bundesministerium der Finanzen (1982) - in der zur Diskussion stehenden Reform der Gewerbesteuer diese additive Variante, als kommunale Steuer ausgestaltet, anstreben.

Die Entfernung von der güterorientierten Sichtweise wird auch dann deutlich, wenn mittels additivem Verfahren nach Gütern bzw. deren Verwendungszweck differenziert besteuert werden soll. Geht man davon aus, daß Unternehmen eine ganze Palette von Produkten herstellen, die nun unterschiedlich zu besteuern wären, müßten bei konsequenter Ausgestaltung, Wertschöpfungsanteile in Form einer Kostenträgerrechnung den Gütern zugeordnet werden. Erst darauf wären die Steuersätze anwendbar. Als praktisches Vorgehen ist dies wohl kaum ernsthaft in Erwägung zu ziehen. Eine Steuerdifferenzierung nach Unternehmen bzw. Wirtschaftszweigen pauschaliert würde auf der anderen Seite ähnliche steuerlich bedingte Wettbewerbsverzerrungen in horizontaler Richtung hervorrufen, wie sie bei einer Brutto-Allphasensteuer in vertikaler zu verzeichnen sind.

Es sei noch darauf hingewiesen, daß die theoretische Bedeutung der additiven Erhebungsmethode weitaus größer ist als ihre praktische Relevanz. Zwei der wenigen hier anzuführenden Beispiele sind die revidierte Fassung des japanischen Umsatzsteuergesetzes von 1951 und die 1975 im US-Bundesstaat Michigan (wieder) eingeführte Single Bu-

${ }^{21}$ Vgl. hierzu auch ITO [1955], S. 451 f. und PoHMER [1977], S. $493 \mathrm{ff}$. 
siness Tax. In Japan wurde auf Empfehlung der Shoup Komission 1950 eine Umsatzsteuer in das neue Gesetz über lokale Steuern integriert, wobei ursprünglich nur die subtraktive Methode vorgesehen war. Aufgrund eines zweiten Berichts der Komiission, der dem Argument Rechnung trug, daß Unternehmen beim additiven Erhebungsverfahren auf bereits existierende Berechnungen der Gewinnermittlung für die Einkommen- oder Körperschaftsteuer zurückgreifen könnten, änderte man 1951 die Gesetzgebung und räumte Gesellschaftsunternehmen ein Wahlrecht zwischen additiver und subtraktiver Methode ein ${ }^{22}$. Zu einer praktischen Verwirklichung der Steuer in dieser Form kam es freilich nie. Sie wurde zuletzt auf das Jahr 1954 verschoben und schließlich gänzlich abgeschafft. Aus ähnlichen Beweggründen entschied man sich wohl auch in Michigan letztendlich für den additiven Ansatz. Zudem konnte man auf frühere Erfahrungswerte zurückgreifen, denn von 1953 bis 1967 wurde bereits eine modifizierte Form der Umsatzsteuer auf subtraktiver Basis (Business Activities Tax) erhoben, wobei schon damals neben dem Vorumsatzabzugsverfahren eine Optionsmöglichkeit für das additive Verfahren bestand ${ }^{23}$.

\subsection{Die Berechnung der Umsatzsteuerschuld}

Wird die Steuer nach dem subtraktiven Verfahren erhoben, bestehen zwei Möglichkeiten, die über die Vorleistungsbezüge anfallenden Umsatzsteuerbeträge anzurechnen. Zum einen kann nach dem Vorumsatzabzugsverfahren zunächst die Differenz zwischen (Netto-) Umsatz und (Brutto-) Vorleistungen gebildet werden, worauf anschließend der Steuersatz anzuwenden ist, oder aber man subtrahiert nach dem Vorsteuerabzugsverfahren die auf Vorleistungskäufe anfallenden Vorsteuern direkt von der auf den (Netto-) Umsatz zu entrichtenden BasisUmsatzsteuerschuld. Beide Verfahren sind identisch, solange alle Güter einem einheitlichen Steuersatz unterliegen. Geht man davon aus, daß beim Vorumsatzabzugsverfahren eine Steuer auf Vorleistungen $T^{V}$ als Aufwandsposten in unser stilisiertes Produktionskonto eingeht, und damit (cet. par.) auch den Netto-Umsatz um denselben Betrag erhöht

22 Vgl. auch ITO ([1955], S.448 und S. $461 \mathrm{ff}$.)

${ }^{23}$ Siehe hierzu auch LiNdholm ([1980], S. 19 f.) oder EBEL ([1972], S. 29 ff.). 
$\left(\tilde{U}=U+T^{V}\right)$, während beim Vorsteuerabzugsverfahren die Preiskalkulation der Unternehmer, bedingt durch die direkte Anrechnung der Vorsteuern, nicht beeinflußt wird, können - formal betrachtet - beide Verfahren ineinander überführt werden:

$$
\begin{array}{rlrl} 
& \text { Vorumsatzabzugsverfahren: } & T_{u}^{U} & =\tau\left(\tilde{U}-\left(V+T^{V}\right)\right) \\
& =\tau\left(U+T^{V}-V-T^{V}\right) \\
& \text { Vorsteuerabzugsverfahren: } & T_{s}^{U} & =\tau U-\tau V .
\end{array}
$$

Erst bei einer differenzierten Besteuerung zeigt sich eine abweichende Wirkung des Vorsteuerabzugsverfahrens gegenüber dem Vorumsatzabzugsverfahren. Greifen wir zur Veranschaulichung das numerische Beispiel von Figur 3 auf.

Figur 5: Vorumsatzabzugsverfahren

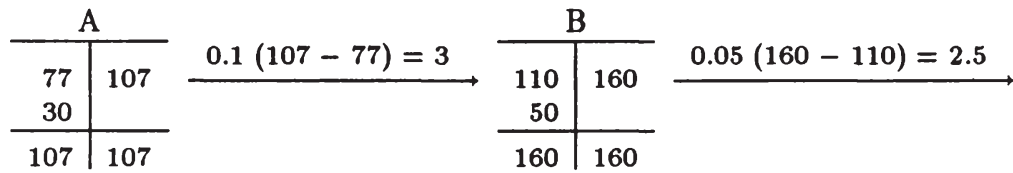

$$
\begin{aligned}
& \begin{array}{c|ccc}
\multicolumn{2}{c}{\mathrm{C}} & & \text { Endverbrauch } \\
\hline \begin{array}{c}
162.5 \\
50
\end{array} & 212.5 & 0.1(212.5-162.5)=5 & 217.5 \\
\cline { 1 - 2 } & & &
\end{array}
\end{aligned}
$$

In Figur 5 wurde angenommen, daß auf alle Umsätze der Unternehmen A vorgelagerten Produktionsstufen ein einheitlicher Steuersatz von $10 \%$ angewendet wird. $\mathrm{Zu}$ diesem Satz werden auch die Umsätze von A und $\mathrm{C}$ besteuert, während Unternehmen B einem reduzierten Steuersatz von $5 \%$ unterliegt. Die Verbuchung der Größen erfolgt analog zu Figur 3. Es fällt sofort ins Auge, daß auch nach dieser Methode die Besteuerung der Wertschöpfung sehr ausgeprägt in Erscheinung tritt. Gleich dem additiven Verfahren entspricht die Umsatzsteuerschuld jedes Unternehmens dem Produkt aus Wertschöpfung und Steuersatz. 
Die eher institutionelle Ausrichtung des Vorumsatzabzugsverfahrens zeigt sich auch in einer anderen Richtung. Produziert ein Unternehmen mehrere Güter, die unterschiedlich zu besteuern sind, müßten die Vorleistungen zweckgebunden verrechnet werden, was selbst bei Ländern mit ausgeprägtem Buchführungssystem nicht ohne weiteres durchführbar ist.

Figur 6: Vorsteuerabzugsverfahren

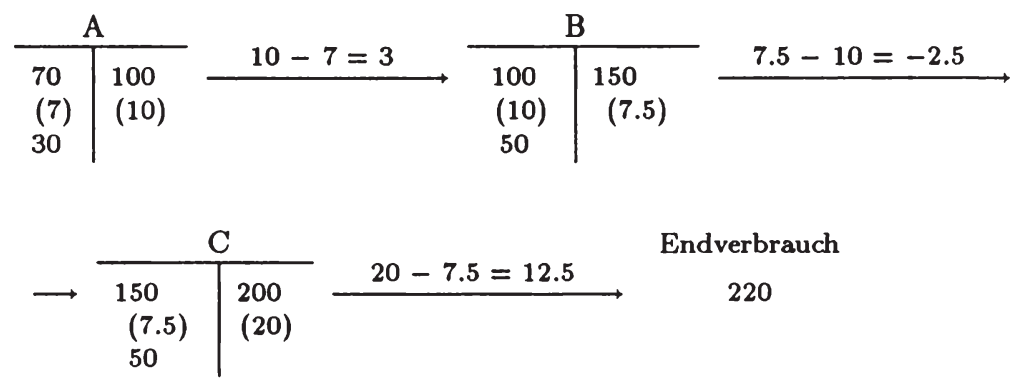

Zum besseren Verständnis des Vorsteuerabzugsverfahrens wurde die Ausweistechnik in Figur 6 gegenüber Figur 5 leicht modifiziert, die Netto-Größen sowie die auf die Unternehmen angewandten Steuersätze dagegen beibehalten. Da beim Vorsteuerabzugsverfahren die Steuerbeträge direkt miteinander verrechnet werden, empfiehlt es sich, nicht nur die Umsätze, sondern auch die Vorleistungen netto, d. h. ohne Umsatzsteuern, auszuweisen. Die Steuerbeträge stehen deshalb als Klammerausdrücke unter den entsprechenden Nettogrößen. So ist beispielsweise mit den Vorleistungseinkäufen des Unternehmens A in Höhe von (netto) $70 \mathrm{DM}$ eine Steuerzahlung von $7 \mathrm{DM}$ verbunden, mit den Umsatzerlösen von (netto) $100 \mathrm{DM}$ ein steuerbedingter zusätzlicher Zahlungseingang von $10 \mathrm{DM}$. Die Umsatzsteuerschuld (3 DM) besteht gerade aus der Differenz $\mathrm{zwischen}$ von nachgelagerten Unternehmen eingenommenen und an vorgelagerte Unternehmen entrichteten Steuerbeträge. Zum Ausgleich der Kontenseiten müßte lediglich die Steuerzahllast als weiterer Zahlungsausgang verbucht werden. 
Aus der zu leistenden Umsatzsteuerschuld ist beim Vorsteuerabzugsverfahren (und bei differenzierten Steuersätzen) die indirekte Besteuerung der Wertschöpfung nicht mehr unmittelbar ersichtlich, sobald unterschiedliche Steuersätze zur Anwendung kommen. Die Steuer ist viel ausgeprägter auf die Güter bezogen. Ein Vergleich beider Verfahren läßt dies erkennen: Die steuerbedingte Werterhöhung ist beim Vorsteuerabzugsverfahren größer; sie entspricht dem auf den Nettoumsatz des letzten Unternehmens angewandten Steuersatz.

Beim Vorumsatzabzugsverfahren ist dies nicht der Fall. Zwar beträgt auch hier der für das letzte Unternehmen relevante Steuersatz $10 \%$, das niedriger besteuerte vorgelagerte Unternehmen B beeinflußt jedoch die Steuerbelastung des Endprodukts. Letztere entspricht dem gewichteten arithmetischen Mittel aus Steuersätzen und Wertschöpfung. Mit dem Vorumsatzabzugsverfahren ist es somit nicht möglich, allein durch die Wahl der Steuersätze die Belastung der Güter exakt festzulegen. Das Vorsteuerabzugsverfahren hingegen besitzt diese Eigenschaft. Man müßte sich nur vorstellen, daß auch Unternehmen A und B Güter an den Endverbrauch liefern. Diese werden genau zu demjenigen Steuersatz steuerlich belastet, der für das jeweils produzierende Unternehmen relevant ist.

Damit bereitet auch eine Kombination des Vorsteuerabzugsverfahrens mit einer Grenzausgleichsteuer auf Importgüter keinerlei Anpassungsprobleme, was die Höhe des Einfuhrumsatzsteuersatzes anbelangt. $\mathrm{Er}$ ist identisch mit dem Steuersatz, der auf das inländische Gut angewandt wird. Da beim Vorumsatzabzugsverfahren die endgültige Steuerbelastung der Güter nicht erkennbar ist, stellt die äquivalente Besteuerung von inländischen und gleichartigen importierten Waren eine kaum zu lösende Aufgabe dar.

Das Vorumsatzabzugsverfahren zeigt sich somit aus den im vorigen Abschnitt genannten Gründen der additiven Methode unterlegen, wenn die Besteuerung auf Einkommensgrößen gerichtet ist, und aus den oben genannten, dem Vorsteuerabzugsverfahren, falls eine nach Gütern differenzierte Besteuerung erfolgen soll. Es dürfte deshalb kaum verblüffen, daß dieses Verfahren selten praktiziert wird ${ }^{24}$.

\footnotetext{
${ }^{24}$ Wie im vorigen Abschnitt erwähnt, sah Japans Umsatzsteuer ursprünglich
} 


\subsection{Die unmittelbare Belastungswirkung}

Neben den bisher erläuterten Klassifizierungsmerkmalen charakterisiert man die Umsatzsteuer anhand der ökonomischen Zielgröße, die letztendlich besteuert werden soll. Im wesentlichen kommen hier das Bruttosozialprodukt, das Volkseinkommen oder der gesamtwirtschaftliche Konsum in Betracht. Wie schon an einigen Stellen - vor allem in den letzten beiden Teilabschnitten - bemerkt wurde, eignen sich bestimmte Formen der Umsatzsteuer zwar besser als andere, ein spezifisches Besteuerungsziel zu erreichen, rein prinzipiell können jedoch fast alle Umsatzsteuertypen durch eine entsprechende (mehr oder weniger komplexe) Ausgestaltung die verschiedensten gesamtwirtschaftlichen Aggregate erfassen. Zum Beispiel wäre es mit der typischerweise nur den Konsum besteuernden einphasigen Einzelhandelsteuer auch möglich, durch Registrierung der Investitionsgüter herstellenden Wirtschaftszweige die inländische Absorption zu besteuern. Gleichermaßen könnte die einphasige Produktionsteuer in diese Richtung modifiziert werden, sofern die Produktionsstruktur einer Volkswirtschaft die Unterscheidung zwischen Investitions- und Konsumgütern erlaubt.

Bei Nettoumsatzsteuern erreicht man die unterschiedlichen ZielgröBen durch Erweiterung bzw. Kürzung der (materiellen) Bemessungsgrundlage. Zur Darstellung der davon ausgehenden Besteuerungseffekte wollen wir nicht mehr auf das bisher verwendete Kontensystem in einzelwirtschaftlicher Sicht zurückgreifen, sondern ein alternatives volkswirtschaftliches Gesamtrechnungswerk - die Input-OutputTabelle - heranziehen. Während sich die stilisierten Produktionskonten sehr gut dazu eignen, die grundsätzlichen Unterschiede in der Besteuerungstechnik der verschiedenen Umsatzsteuertypen auf Unternehmensebene herauszustellen, erfordert die gesamtwirtschaftliche Betrachtungsweise eine Darstellungsform, die sowohl volkswirtschaftliche Aggregatsgrößen als auch die wirtschaftliche Verflechtungsstrukur ausweist. Hierfür bietet sich die Input-Output-Tabelle geradezu an.

Vernachlässigt man zur Vereinfachung außenwirtschaftliche Bezie-

ein Vorumsatzabzugsverfahren vor. Ihre Ausgestaltung ist schematisiert in BRONFENBRENNER [1950], S. 306 dargestellt. Eine ausführlichere Beschreibung der Single Business Tax Michigans kann bspw. SUlLIVAN [1965], S. 298 ff. oder LINDHOLM [1975], S. 92 f. entnommen werden. 
Figur 7: Aufbau der Input-Output-Tabelle

\begin{tabular}{|c|c|c|c|c|}
\hline \multicolumn{2}{|c|}{ Vorleistungsverflechtung } & $\begin{array}{l}\text { Konsum } \\
\qquad q_{i} C_{i}\end{array}$ & $\begin{array}{l}\text { Investitionen } \\
\qquad q_{i} I_{i}^{b r}\end{array}$ & $\begin{array}{l}\text { Summe } \\
\qquad q_{i} Q_{i}\end{array}$ \\
\hline Abschreibungen & $D_{i}$ & & & \\
\hline Löhne und Gehälter & $L G_{i}$ & & & \\
\hline Nettobetriebsüberschu $B$ & $N B U_{i}$ & & & \\
\hline
\end{tabular}

hungen und indirekte Unternehmensteuern, so erübrigt sich eine Unterscheidung von Sozialprodukt- und Inlandsproduktgrößen sowie deren Bewertung zu Marktpreisen bzw. Faktorkosten. Die Grundstruktur, der auf diese Weise simplifizierten Input-Output-Tabelle, zeigt Figur 7. Ihre Interpretation und der Aussagegehalt der einzelnen Tabellengrößen wird als bekannt unterstellt ${ }^{25}$. Es sei jedoch betont, daß der Ausweis der einzelnen Felderwerte güterorientiert ist und nicht nach Unternehmen geordnet erfolgt. So ist beispielsweise im Wertschöpfungsquadranten $N B U_{i}$ nicht der Nettobetriebsüberschuß des $i$-ten Unternehmens bzw. der $i$-ten Unternehmensgruppe, sondern der Nettobetriebsüberschuß, der bei der Produktion der $i$-ten Gütergruppe erwirtschaftet wurde. Da die einzelnen Größen sinngemäß mit den entsprechenden Posten der zuvor benutzen Produktionskonten übereinstimmen, wurde die Nomenklatur im wesentlichen beibehalten, mit der Ausnahme, daß Wertgrößen nun explizit als Produkt von Menge und Preis dargestellt sind und in der gesamtwirtschaftlichen Betrachtungsweise der Suffix $i$ die Gütergruppen unterscheidet.

${ }^{25}$ Einerseits eignet sich der Aufbau einer Input-Output-Tabelle hervorragend für die einfache Demonstration der Belastungswirkung, andererseits können die diesen Tabellen zugrundeliegenden speziellen Ausweiskonzepte, bspw. der Ausweis von Produktions- und Verbrauchswerten, das verwendete Preiskonzept, die Behandlung besonderer Wirtschaftsbereiche usw. einige Verwirrung stiften. Konzentrieren wir uns deshalb auf den grundlegenden Kreislaufgedanken und betrachten die tabellenspezifische Ausweisproblematik als nicht relevant. 
Da die Tabelle zeilenweise den jeweiligen Verwendungszweck der Güter angibt, wollen wir die Zeilensumme $q_{i} Q_{i}$ als Gesamtumsatz $\left(U_{i}\right)$ des Gutes interpretieren ${ }^{26}$. Er kann aus Vorleistungsverkäufen für die Produktion anderer Güter $\left(\sum_{j} q_{i} A_{i j}\right)$, aus Verkäufen für konsumptive Zwecke $\left(q_{i} C_{i}\right)$ oder für investive Zwecke $\left(q_{i} I_{i}^{b r}\right)$ bestehen. Aus der Gleichheit von Spalten- und Zeilensummen der Tabelle

$$
\sum_{j} q_{j} A_{j i}+D_{i}+L G_{i}+N B U_{i}=\sum_{j} q_{i} A_{i j}+q_{i} C_{i}+q_{i} I_{i}^{b r} \quad i, j=1, \ldots, n
$$

folgt unmittelbar, daß die gesamtwirtschaftliche Bruttowertschöpfung mit dem Bruttosozialprodukt übereinstimmt. Ferner führt die Summe der vorangehenden Gleichung über alle Spalten bzw. Zeilen hinweg,

$$
\begin{aligned}
\sum_{i}\left(\sum_{j} q_{j} A_{j i}+D_{i}+L G_{i}+N B U_{i}\right) & =\sum_{i}\left(\sum_{j} q_{i} A_{i j}+q_{i} C_{i}+q_{i} I_{i}^{b r}\right) \\
\sum_{i}\left(D_{i}+L G_{i}+N B U_{i}\right) & =\sum_{i}\left(q_{i} C_{i}+q_{i} I_{i}^{b r}\right)
\end{aligned}
$$

zu der Darstellung des Bruttosozialprodukts von der Entstehungs- und der Verwendungsseite.

Eine Umsatzsteuer (mit einheitlichem Steuersatz) nach der additiven Methode auf alle Wertschöpfungskomponenten erhoben, belastet somit das gesamte Bruttosozialprodukt von der Entstehungsseite. Über die Verwendungsseite erreicht man mit der subtraktiven Methode das gleiche Belastungsziel, indem der Umsatz abzüglich der Aufwendungen an Vorleistungen als Bemessungsgrundlage zugrundegelegt wird:

${ }^{26}$ Streng genommen ist diese Interpretation nicht zulässig, da nur Produktionsund Verbrauchswerte ausgewiesen sind, ungeachtet, ob damit eine Markttransaktion verbunden ist, oder nur eine unternehmensinterne Güterbewegung stattfindet. Für die nachfolgenden Überlegungen ist diese „Fehlinterpretation" bis auf eine Ausnahme unproblematisch. Sie wird an späterer Stelle aufgegriffen. 


$$
\begin{aligned}
\sum_{i}\left(q_{i} Q_{i}-\sum_{j} q_{j} A_{j i}\right) & =\sum_{i}\left(\sum_{j} q_{i} A_{i j}+q_{i} C_{i}+q_{i} I_{i}^{b r}-\sum_{j} q_{j} A_{j i}\right) \\
& =\sum_{i}\left(q_{i} C_{i}+q_{i} I_{i}^{b r}\right)
\end{aligned}
$$$$
\text { wegen } \quad \sum_{i} \sum_{j} q_{j} A_{i j}=\sum_{i} \sum_{j} q_{i} A_{j i} \text {. }
$$

Man spricht in diesem Fall von einer Umsatzsteuer vom Bruttosozialproduktstyp.

Eine nach der additiven Methode nur auf die Löhne und Gehälter sowie den Nettobetriebsüberschuß erhobene Steuer trifft hingegen das Nettosozialprodukt bzw. Volkseinkommen. Sie entspricht einer unmittelbar bei den Unternehmen eingezogenen proportionalen Einkommensbesteuerung. Diese Umsatzsteuer vom Einkommenstyp wird nach der subtraktiven Methode durch Kürzung der ursprünglichen Bemessungsgrundlage um die Abschreibungen erzielt, was die folgende Umformung zeigt:

$$
\begin{aligned}
\sum_{i}\left(L G_{i}+N B U_{i}\right) & =\sum_{i}\left(q_{i} Q_{i}-\sum_{j} q_{j} A_{j i}-D_{i}\right) \\
& =\sum_{i}\left(q_{i} Q_{i}-\sum_{j} q_{i} A_{i j}-D_{i}\right) .
\end{aligned}
$$

Ersetzt man auf der rechten Seite dieser Gleichung $q_{i} Q_{i}$ durch die einzelnen Zeilenkomponenten, so ist wegen $I_{i}^{n}\left(=I_{i}^{b r}-D_{i}\right)$ über

$$
\begin{aligned}
\sum_{i}\left(q_{i} Q_{i}-\sum_{j} q_{i} A_{i j}-D_{i}\right) & =\sum_{i}\left(\sum_{j} q_{i} A_{i j}+q_{i} C_{i}+q_{i} I_{i}^{b r}-D_{i}\right) \\
& =\sum_{i}\left(q_{i} C_{i}+q_{i} I_{i}^{n}\right)
\end{aligned}
$$

zu erkennen, daß im Endeffekt der Konsum und die Nettoinvestitionen besteuert werden. 
Es sei jedoch darauf hingewiesen, daß diese einfache Umformung nur für eine geschlossene Volkswirtschaft gilt. In offenen Volkswirtschaften werden Teile des im Inland erwirtschafteten Einkommens für ausländische Kapitalanlagen benutzt und inländische Investitionen zum Teil durch ausländische Mittel finanziert. Das Volkseinkommen von der Verwendungsseite her zu besteuern, gestaltet sich damit wesentlich komplexer.

$\mathrm{Zu}$ einer Umsatzsteuer vom Konsumtyp gelangt man nach der subtraktiven Methode schließlich dadurch, daß anstelle der Abschreibungen die Investitionsgüter insgesamt neben den Vorleistungen die Bemessungsgrundlage reduzieren. Formal:

$$
\begin{aligned}
\sum_{i}\left(q_{i} Q_{i}-\sum_{j} q_{j} A_{j i}-q_{i} I_{i}^{b r}\right) & =\sum_{i}\left(\sum_{j} q_{i} A_{i j}+q_{i} C_{i}+q_{i} I_{i}^{b r}-\right. \\
& \left.-\sum_{j} q_{j} A_{j i}-q_{i} I_{i}^{b r}\right) \\
& =\sum_{i} q_{i} C_{i}
\end{aligned}
$$

Die hierzu äquivalente Bemessungsgrundlage für das additive Verfahren - die gesamte Wertschöpfung abzüglich der Investitionsgüter hat ebenso nur in einer geschlossenen Volkswirtschaft Bestand.

Nach dieser Betrachtung zeigt sich, daß beide Erhebungsverfahren grundsätzlich auf alle drei Besteuerungstypen ausgerichtet werden können. Bei einer Umsatzsteuer vom Einkommenstyp ist jedoch (schon auf diesem hohen Abstraktionsniveau) der additiven Methode, bei einer Umsatzsteuer vom Konsumtyp dem subtraktiven Erhebungsverfahren der Vorzug zu geben. Berücksichtigt man weiterhin, daß das subtraktive Verfahren nur an Markttransaktionen ansetzen kann und damit selbsterstellte Anlagen und Vorratsveränderungen nicht bzw. in einer anderen Periodizität ${ }^{27}$ erfaßt, ist das additive Verfahren auch bei einer Umsatzsteuer vom Bruttosozialproduktstyp vorzuziehen.

${ }^{27}$ Güter, die geplant oder unfreiwillig auf Lager produziert werden, erhöhen zum Zeitpunkt ihrer Fertigstellung das Bruttosozialprodukt, werden jedoch erst zum Zeitpunkt ihrer Veräußerung besteuert. 


\subsection{Die Besteuerung des Außenhandels}

Eng mit der intendierten Belastungskonzeption ist die steuerliche Behandlung des grenzüberschreitenden Güterverkehrs verbunden. Die Notwendigkeit für eine gesonderte Steuerregelung stellt sich explizit dann, wenn die Umsatzsteuer an die Verwendungseite des Sozialprodukts anknüpft. Eine direkt auf die Wertschöpfungskomponenten erhobene Umsatzsteuer vom Bruttosozialprodukts- oder Einkommenstyp bedarf offensichtlich keiner zusätzlichen Besteuerung der Exporte und/ oder Importe. In beiden Fällen soll und wird das im Inland erwirtschaftete Einkommen (zuzüglich bzw. ohne Abschreibungen) belastet, ganz gleich, ob es durch die Produktion von Exportgütern oder von heimisch verwendeten Gütern entstand. Damit sind jedoch implizit alle inländisch erzeugten Güter - insbesondere auch Exporte - mit der Umsatzsteuer belastet, Importgüter hingegen nicht.

Eine Umsatzsteuer gleichen Typs, die nach dem subtraktiven Verfahren erhoben wird, müßte entsprechend gleichfalls die Exporte in die Besteuerung mit einschließen und Importe im Gegenzug freistellen, um mit der Belastungskonzeption kompatibel zu sein ${ }^{28}$. Da in beiden Fällen diejenigen Güter besteuert werden, die ihren Ursprung im besteuernden Land haben, spricht man von einer Besteuerung nach dem Ursprungslandprinzip.

Diametral hierzu werden bei einer Besteuerung nach dem Bestimmungslandprinzip Exporte von der Umsatzsteuer befreit - wobei die durch den Kauf von Zwischenprodukten anfallende Steuer voll anrechnungsfähig bleibt - und Importe einer Einfuhrumsatzsteuer unterzogen, deren Steuersätze identisch sind mit denen gleichartiger inländischer Produkte. Dieses Prinzip bildet die natürliche Ergänzung einer allgemeinen Verbrauchsteuer. Eine Umsatzsteuer vom Konsumtyp muß sowohl inländische als auch importierte Konsumgüter erfassen, wenn der gesamte private Konsum gleichmäßig besteuert werden soll. Der Steuerzugriff erfolgt hierbei in der Regel direkt an der Landesgrenze. Da dort die Importe unmöglich nach ihrem Verwendungszweck differenzierbar sind, werden grundsätzlich alle Importgüter mit Einfuhrumsatzsteuer belastet. Im Zuge der Ermittlung der Umsatzsteuer-

\footnotetext{
${ }^{28}$ Siehe auch POHMER [1979], S. 254 f.
} 
schuld verbleiben - über die Anrechnung der Vorleistungsbezüge und Investitionsgüterkäufe bzw. der darauf angefallenen Steuerbeträge (ungeachtet der regionalen Herkunft der Güter) - automatisch nur die unmittelbar an den privaten Konsum gelieferten Importe mit der Umsatzsteuer behaftet.

Die logische Zuordnung beider Prinzipien zu bestimmten Umsatzsteuertypen kann nicht immer durchgehalten werden. So wird beispielsweise innerhalb der EG-Mitgliedstaaten das Bestimmungslandprinzip quasi an den Zollgrenzen durchgeführt und kontrolliert. Mit dem für 1993 geplanten Wegfall der Grenzen entzieht man dem Bestimmungslandprinzip damit die bisherige praktische Durchführungsgrundlage. Eine Verlagerung der steuerlichen Erfassung von Importen bzw. Exporten in die Unternehmen erhöht nicht nur den Verwaltungsaufwand beträchtlich, sondern reduziert auch die Kontrollmöglichkeiten. Aus diesem Grund wird eine Umstellung auf das Ursprungslandprinzip innerhalb der EG-Staaten - erweitert um bestimmte Steuerausgleichsverfahren $^{29}$ - diskutiert, obwohl die Staaten einheitlich eine Umsatzsteuer vom Konsumtyp praktizieren. Solange jedoch die national zur Anwendung kommenden Steuersätze zwischen den Ländern sehr differieren, würde die Umstellung auf das Ursprungslandprinzip starke internationale Wettbewerbsverzerrungen auslösen. Da weiterhin bis 1993 keine Angleichung der Steuersätze zu erwarten ist, wird geplant, das Bestimmungslandprinzip trotz der zuvor erwähnten Nachteile für eine Übergangszeit von vier Jahren (bis einschließlich 1996) grundsätzlich beizubehalten, wobei für einige, ohne eine Grenzkontrolle schwer erfaßbare Umsätze - bspw. bestimmte Direktimporte der privaten Haushalte - Sonderregelungen gelten ${ }^{30}$.

Weniger aus praktischen Erwägungen heraus, sondern aufgrund besteuerungstechnischer Probleme ist es unmöglich, das Bestimmungslandprinzip bei einer Umsatzsteuer vom Konsumtyp konsequent an-

${ }^{29}$ Für eine Beschreibung der zur Diskussion stehenden Verfahren und der damit verbundenen Problematik siehe auch EUROPEAN PARLIAMENT [1989].

${ }^{30} \mathrm{Zu}$ Einzelheiten siehe u. a. STEPPERT [1990]. Es sei in diesem Zusammenhang noch darauf hingewiesen, daß auch in der momentan (noch) gültigen Ausgestaltung der Umsatzsteuer Ausnahmen zum Bestimmungslandprinzip bestehen. Man denke hier vor allem an Reiseimportleistungen, die ebenfalls schwer zu erfassen sind. 
zuwenden, wenn bei letzterer die Berechnung der Umsatzsteuerschuld nach der Vorumsatzmethode erfolgt und nach Gütern differenzierte Steuersätze erhoben werden. Wie aus Teilabschnitt (4) hervorgeht, ist die effektive Steuerbelastung der Konsumgüter nicht allein von den zuletzt auf die Güter angewandten Steuersätzen abhängig, sondern ebenso von der Höhe der Wertschöpfung vorgelagerter Produktionsstufen einschließlich der darauf angewandten Steuersätze. Wie hoch sollte nun der Steuersatz für ein direkt vom Einzelhandel an den privaten Endverbrauch geliefertes Importgut gewählt werden, damit keine steuerlich bedingte Preisdiskriminierung zwischen heimisch produziertem und importiertem Gut entsteht? Die gleiche Frage stellt sich natürlich auch für Importgüter, die als Vorleistungen in Unternehmen eingesetzt werden und dabei mit inländischen Produkten konkurrieren.

\section{Das bundesdeutsche Umsatzsteuersystem}

Nach den vorangehenden Kriterien kann die in der Bundesrepublik praktizierte Umsatzsteuer kurz als Netto-Allphasenumsatzsteuer vom Konsumtyp charakterisiert werden, wobei die Berechnung der Umsatzsteuerschuld nach dem Vorsteuerabzugsverfahren erfolgt und hinsichtlich des Außenhandels das Bestimmungslandprinzip angewendet wird. Wir wollen in diesem Abschnitt etwas näher auf die Grundzüge der umsatzsteuerrechtlichen Regelungen eingehen, wobei den Steuerbefreiungen ein besonderer Stellenwert eingeräumt wird. Sonderbestimmungen, wie beispielsweise die Besteuerung der Kleinunternehmer, die Anwendung von Durchschnittsteuersätzen für land- und forstwirtschaftliche Betriebe und deren Kürzungsansprüche, die Vorsteuerpauschalierung, die Ermittlungsverfahren bei teilweisem Ausschluß vom Vorsteuerabzug usw., werden hingegen nicht behandelt.

Steuersubjekt und damit Steuerschuldner ist nach $\S 2$ UStG prinzipiell der Unternehmer ${ }^{31}$. Als Unternehmer gilt hierbei jede natürliche oder juristische Person, die eine gewerbliche oder berufliche Tätigkeit zum Zweck der Einnahmeerzielung selbständig ausübt. Die Absicht Gewinne zu erzielen muß nicht vorliegen. Selbständigkeit ist für natürliche

${ }^{31}$ Nur bei Importen können auch Nichtunternehmer Steuerschuldner sein. 
Personen analog zum Einkommen- und Gewerbesteuerrecht definiert wenn Personen nicht derart in ein Unternehmen eingegliedert sind, daß sie hinsichtlich von Ort, Zeit und Art der Tätigkeit weisungsgebunden handeln ( 2 Abs. 2 Nr. 1 UStG). Nach dieser Abgrenzung werden somit nicht nur Gewerbetreibende und Freiberufler, sondern auch Land- und Forstwirte und Vermieter begrifflich erfaßt. Bei juristischen Personen darf keine finanzielle, wirtschaftliche und organisatorische Eingliederung in ein anderes Unternehmen vorliegen ( $\$ 2$ (2) 1 UStG). Im Falle von Organschaften ist nur der Organträger Steuerschuldner.

Das Unternehmen selbst umfaßt die gesamte gewerbliche und berufliche Tätigkeit des Unternehmers. Der umsatzsteuerrechtliche Unternehmensbegriff ist hierbei nicht räumlich begrenzt. Er bezieht sich auch auf Unternehmensteile, die im Außengebiet liegen.

In der nachfolgenden Figur 8 ist der Ablauf der Umsatzsteuerermittlung strukturiert dargestellt. Für alle Unternehmensvorgänge mit Umsatzvermutung ist zunächst zu prüfen, ob ein Leistungsaustausch - in Form von Leistung und Gegenleistung (Entgelt) - an unternehmensfremde Dritte stattfindet. Nur dann handelt es sich um Umsätze im steuerrechtlichen Sinn. Sogenannte Innenumsätze, d. h. zwischen Unternehmensteilen oder Unternehmen derselben Organschaft, werden ebenso wie Mittelzuflüsse ohne willentliche Wertabgabe (bspw. Erbschaften) als Nichtumsätze klassifiziert ${ }^{32}$.

Die steuerbaren Umsätze werden nach $\S 1$ Abs. 1 UStG in vier Umsatzarten eingeteilt: die Lieferungen und Leistungen des Unternehmens einschließlich der Arbeitnehmerversorgung, den Eigenverbrauch (Privatentnahme), den Gesellschafterverbrauch und die Einfuhr. Ein Umsatz ist jedoch nur dann steuerbar, wenn alle in diesem Absatz genannten Kriterien erfüllt sind. Hierzu muß der Umsatz

- im Rahmen des Unternehmens

- nicht im Außengebiet und

- nicht unentgeltlich

ausgeführt werden. Ist nur eines der Kriterien verletzt, so ist der Umsatz nicht steuerbar.

Mit dem Vorliegen steuerbarer Umsätze allein, entsteht noch keine

${ }^{32}$ Siehe hierzu auch DZIADKOWSKI [1986], S. $46 \mathrm{f}$. 
Figur 8: Ermittlung der Umsatzsteuerschuld

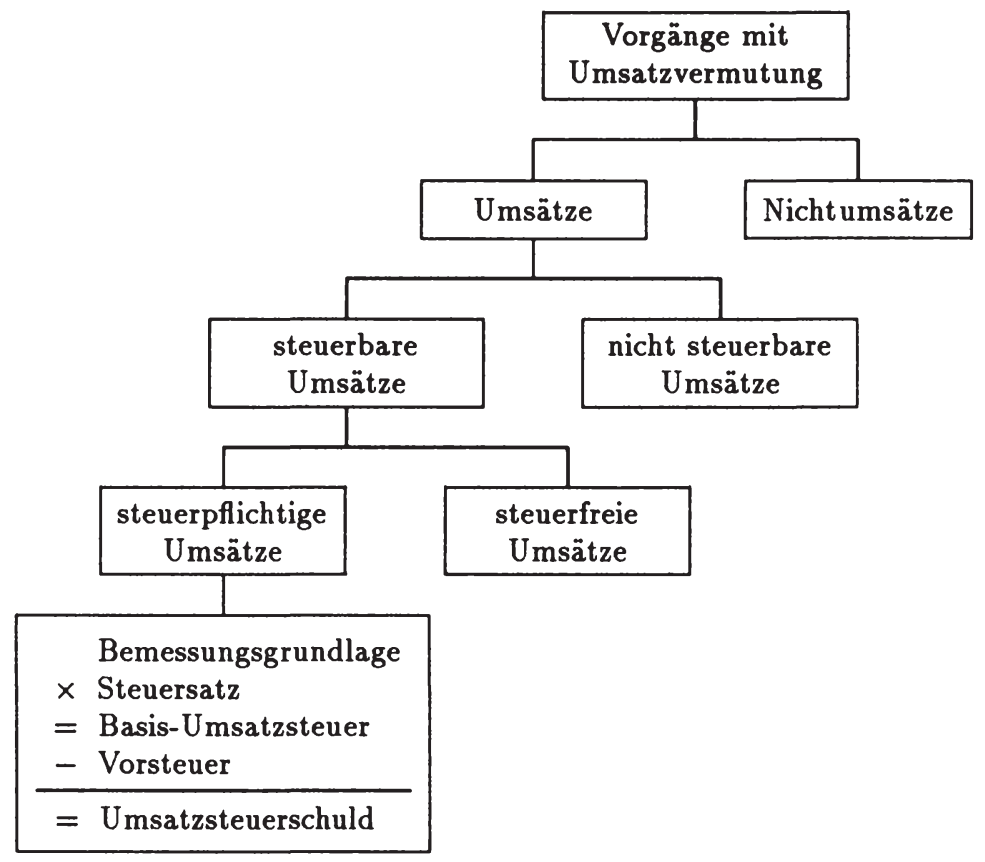

Quelle: DzIADKowsKI [1986], S. 10

Umsatzsteuerschuld. Ein Umsatz kann steuerbar und nicht steuerpflichtig sein, wenn er zu den in $\S 4$ UStG genannten steuerbefreiten Umsatzarten zählt. Auf der anderen Seite kann auch ein dem Wesen nach steuerbefreiter Umsatz steuerpflichtig werden, falls der Unternehmer für die Steuerpflicht optiert. Wir wollen auf die mit der Steuerbefreiung zusammenhängenden Regelungen am Ende des Abschnitts zurückkommen.

Für steuerpflichtige Umsätze erfolgt die Berechnung der Basis-Umsatzsteuer durch Anwendung eines Steuersatzes auf die Bemessungsgrundlage. Der Regelsteuersatz beträgt 14 Prozent, für bestimmte Umsatzarten wird jedoch ein ermäßigter Steuersatz von 7 Prozent an- 
gewandt ( $\$ 12(1)$ UStG). Daneben existieren zwei Durchschnittsteuersätze (5 und 8 v. H.) $)^{33}$ für die Land- und Forstwirtschaft ( $\$ 24$ UStG). Die Bemessungsgrundlage bildet in der Regel das Entgelt, d. h. die tatsächlichen Aufwendungen des Leistungsempfängers. Nur bei fehlender (bspw. Eigenverbrauch) oder unangemessen niedriger (bspw. Arbeitnehmer- oder Gesellschafterverbrauch) Entgeltvereinbarung können sog. Ersatzwerte nach § 14(4) UStG herangezogen werden.

Von dieser Basis-Umsatzsteuer einer bestimmten Periode können alle in derselben Periode vom Unternehmer entrichteten Vorsteuern abgezogen werden. Die Voraussetzung der Abziehbarkeit ist hierbei nach $\S 15$ (1) 1 UStG an folgende Kriterien gebunden: Die Vorsteuer muß gesondert ausgewiesen (in Rechnung gestellt) sein, Lieferer und Empfänger der Leistung müssen Unternehmer, die Leistung im Rahmen der Unternehmen gegen Entgelt durchgeführt worden sein. Für die Abzugsberechtigung der Vorsteuer spielt es jedoch keine Rolle, ob die damit verbundenen Vorleistungen in derselben Periode verbraucht wurden - d. h. tatsächlich in den Produktionsprozeß gelangten -, in welcher der Verkauf der (End-) Leistung erfolgte und damit Basis-Umsatzsteuer erzeugte. Mit anderen Worten, daß Vorleistung und Leistung, für die Vorsteuer und Basis-Umsatzsteuer entstanden, auch im produktionstechnischen Sinn in der gleichen Abrechnungsperiode anfielen. Ein Unternehmer kann auch Vorsteuern für Lieferungen anrechnen, die zunächst auf Lager gehen und erst in einer späteren Periode weiterverarbeitet werden. Ebensowenig ist das Anfallen einer Basis-Umsatzsteuer Voraussetzung für die Anrechenbarkeit der Vorsteuer. Durch Abzug der Vorsteuer von der Basis-Umsatzsteuer ergibt sich die UmsatzsteuerZahllast (Umsatzsteuerschuld) des Unternehmers. Sie kann in manchen Fällen auch negativ sein, und damit eine Steuerrückerstattung darstellen.

Die Berechnung der Umsatzsteuerschuld erfolgt dabei grundsätzlich nach vereinbarten Entgelten, d. h. Umsatzsteuer entsteht in der Abrechnungsperiode, in der die Leistung tatsächlich ausgeführt wurde (§16(1) UStG) und nicht etwa zum Zeitpunkt der Ausstellung der Rechnung oder der Vereinnahmung des Entgelts. Eine Istbesteuerung nach ver-

${ }^{33}$ Zeitlich befristet kommen zwei weitere Durchschnittsätze von 13 bzw. $11 \%$ zur Anwendung ( $\$ 12$ Abs. 1 Nr. 4 und Nr. 5 UStG). 
einnahmten Entgelten wird nur in bestimmten Sonderfällen (geregelt in $\S 20$ UStG) bspw. bei freiberuflicher Tätigkeit gestattet.

Die steuerbefreiten Umsätze sind in $\S 4$ UStG aufgeführt. Da dieser Befreiungskatalog recht stattlich ist und zudem die befreiten Umsatzarten sehr detailliert beschrieben sind, wird er in Kurzform in Anhang 1 wiedergegeben. Grundsätzlich führt die Steuerbefreiung zum Verlust des Vorsteuerabzugsrechts ( $§ 15$ Abs. 2 UStG). Für einige der aufgeführten Umsätze bestehen jedoch Ausnahmen. Aufgrund dieser Sonderregelungen können die Befreiungen nach der steuerlichen Behandlung in vier Gruppen unterteilt werden ${ }^{34}$ :

(1) Steuerbefreiungen mit Vorsteuerabzugsrecht

(2) Steuerbefreiungen mit bedingtem Vorsteuerabzugsrecht

(3) Steuerbefreiungen mit Vorsteuerabzugsmöglichkeit nach Verzicht auf die Steuerbefreiung

(4) Steuerbefreiungen ohne Vorsteuerabzugsrecht infolge absolutem Vorsteuerabzugsverbot.

$\mathrm{Zu}$ den steuerfreien Umsätzen mit Vorsteuerabzugsrecht zählen die in $\S 4 \mathrm{Nr}$. 1-6 genannten Umsatzarten. Es handelt sich hierbei im wesentlichen um Exporte, Veredelungsleistungen sowie Leistungen der Durchfuhr. Da der Unternehmer die Vorsteuer in vollem Umfang geltend machen kann, sind die Umsätze an der Grenze nominal und faktisch nicht mit Umsatzsteuer belastet. Man bezeichnet diesen Typ der Steuerbefreiung deshalb auch als echte Steuerbefreiung.

Die Steuerbefreiungen der Gruppe (2) umfassen typische Umsätze von Banken ( $\$ 4$ Nr. 8a-g UStG) und Versicherungen ( $\$ 4$ Nr. 10a UStG), die prinzipiell nicht zum Vorsteuerabzug berechtigen. Werden sie jedoch unmittelbar in Verbindung mit Exporten an Dritt-Länder - die nicht zur Europäischen Gemeinschaft gehören - ausgeführt, so ist der Vorsteuerabzug gestattet.

Unternehmer können gemäß $\S 9$ UStG das Vorsteuerabzugsrecht für die unter $\S 4 \mathrm{Nr} .8 \mathrm{a}-\mathrm{g}, \mathrm{Nr} .9 \mathrm{a}, \mathrm{Nr} .12, \mathrm{Nr} .13$ und Nr. 19 UStG fallenden Umsatzarten auch dadurch wiedererlangen, indem sie für die Steuerpflicht optieren. Der Verzicht auf die Steuerbefreiung bedarf keiner besonderen Genehmigung, nur der Abnehmer der Leistung muß

\footnotetext{
${ }^{34}$ Vgl. hierzu DZIADKoWSKI [1986], S. 127; RoSE [1987], S. 154.
} 
- als zusätzliche Voraussetzung - ebenfalls Unternehmer sein. Ob die Option zur Steuerpflicht lohnt, hängt u. a. von den jeweiligen Geschäftsverbindungen des Unternehmers ab. Ist der Leistungsempfänger voll zum Vorsteuerabzug berechtigt, stellen sich beide Parteien günstiger, wenn der befreite Unternehmer für die Steuerpflicht optiert. Sind die Erzeugnisse bzw. Leistungen, die der Empfänger seinerseits herstellt, hingegen der Gruppe (4) zurechenbar, so könnte er in diesem Fall die gezahlte Steuer nicht geltend machen. Die Vorleistungen hätten sich für ihn verteuert. Der liefernde Unternehmer würde seine eigene Wettbewerbsposition durch die Option für die Steuerpflicht verschlechtern.

Bei befreiten Umsätzen, die nicht zum Vorsteuerabzug berechtigen (Gruppe (4)), werden die vom Unternehmer gezahlten Vorsteuern automatisch zum Aufwand und fließen in die Preiskalkulation mit ein. Man spricht in diesem Zusammenhang auch von einer Nachholwirkung der Steuer oder einer unechten Steuerbefreiung, da die Umsätze zwar nominal, nicht aber faktisch steuerlich unbelastet sind. Ist der Abnehmer der Leistung ein Unternehmer, kann auch er keine Vorsteuer für diesen Umsatz geltend machen. Die unechte Steuerbefreiung führt somit durch den Abbruch der Vorsteuerabzugskette zu ähnlichen Kaskadeneffekten, wie sie bei Brutto-Allphasensteuern in Abschnitt 2.3 festgestellt wurde. Zwar ermöglicht das Optionsrecht für Umsätze der Gruppe (3) eine teilweise Eindämmung derartiger Kumulationswirkungen, auf der anderen Seite sind diese Umsätze dann dem Regelsteuersatz unterworfen. 


\section{Steuerbefreiungen und Nullsteuersätze}

Ein ungefähres Maß für die internationale Bedeutung der Umsatzsteuer liefert die in Anhang 2 angeführte Tabelle. Sie zeigt, daß mittlerweile rund 46 Länder eine Netto-Allphasenumsatzsteuer erheben. Obwohl die ersten von W. von Siemens [1919] stammenden Vorschläge zur Konzeption eines Nettosystems auf das Vorumsatzabzugsverfahren hinausliefen, kommt bei diesen Ländern ausnahmslos das Vorsteuerabzugsverfahren zur Anwendung. Nach den in den Teilabschnitten (2.4)-(2.6) erwähnten Vorzügen des Vorsteuerabzugsverfahrens gegenüber dem additiven und dem Vorumsatzabzugsverfahren ist dies kaum überraschend. Es gewährleistet - in Reinform - auch bei differenzierten Steuersätzen eine gezielte steuerliche Belastung der Güter und ermöglicht eine adäquate Besteuerung von Importen und Exporten nach dem Bestimmungslandprinzip.

Sehr treffend kann diese Form der Umsatzbesteuerung mit den Worten von WETZLER charakterisiert werden: „A value added tax (VAT) is essentially a national retail sales tax with the tax collected bit by bit as the final product is produced, rather than all at once when it is sold at retail (WeTzler [1979], S. 338)." Wird die Steuer konsequent nach dem Vorsteuerabzugsverfahren erhoben, so entspricht die Steuerbelastung der Güter (-umsätze) auf jeder Produktionsstufe dem gerade angewandten Steuersatz, ungeachtet der Steuersätze, die für vorgelagerte Produktionsstufen relevant waren. Aus den im letzten Abschnitt beschriebenen Grundzügen der Steuerregelungen in der Bundesrepublik war jedoch zu entnehmen, daß bestimmte Güterumsätze steuerbefreit sind, ohne daß ein Recht auf Vorsteuer abzug eingeräumt wird. Der Terminus Befreiung erhält damit eine im Vergleich zu anderen Steuerarten abweichende Bedeutung. Nominell wird zwar ein Steuersatz von Null angewendet, trotzdem bleiben auf befreiten Gütern diejenigen Steuerbeträge haften, die über Vorleistungsbezüge angefallen sind. Ein echter Nullsteuersatz käme nur dann zustande, wenn das entsprechende Unternehmen eine Umsatzsteuer-Rückerstattung in Höhe der gezahlten Vorsteuerbeträge erhielte.

Die auf Seite 42/43 abgedruckte Tabelle 1 läßt erkennen, daß diese Art von (unechten) Befreiungen keineswegs eine Besonderheit der in der Bundesrepublik erhobenen Steuer darstellt. Im Gegenteil, bei den 26 
angegebenen Ländern sind Befreiungen weitaus häufiger gebräuchlich als Nullsteuersätze. Lediglich Großbritannien, Irland und Portugal verwenden letztere in größerem Ausmaß. Bei der Durchsicht von Tabelle 1 ist jedoch zu beachten, daß es eine derart schematisierte Übersicht von Befreiungen und Nullsteuersätzen notwendig macht, bestimmte Sachverhalte $\mathrm{zu}$ verallgemeinern. Beispielsweise sind in der Bundesrepublik die Leistungen der Deutschen Bundespost größtenteils befreit, während die Leistungen der Bundesbahn besteuert werden; in Irland wird nicht auf den gesamten Bekleidungssektor ein Nullsteuersatz angewendet, sondern nur auf Kinderbekleidung und Schuhe; Mexiko besteuert nicht den gesamten Finanzsektor, Versicherungen für die Landwirtschaft und Lebensversicherungen sind befreit; in Chile sind nicht alle medizinischen Leistungen von der Umsatzsteuer befreit, sondern nur diejenigen die bestimmten gesetzlich festgelegten Abgrenzungkriterien entsprechen ${ }^{35}$.

Die Beweggründe für die Einführung von gestaffelten Steuersätzen insbesondere von Befreiungen und Nullsteuersätzen - sind überwiegend sozial- bzw. verteilungspolitischer Natur. Als sozialpolitisch motiviert könnte man die Steuerbefreiungen von meritorischen Gütern betrachten. In der 6. Richtlinie zur Harmonisierung der Umsatzsteuer in den Mitgliedstaaten der EG wird beispielsweise angeregt, daß „Güter“ wie medizinische Leistungen, Leistungen der Post, Leistungen für Bildungszwecke, kulturelle Veranstaltungen und nichtkommerzielle Radiound Fernsehübertragungen von öffentlichem Interesse sind und deshalb nicht besteuert werden sollten.

${ }^{35}$ Vgl. TAIT [1988], S. 52. 
Tabelle 1: Beispiele für die Anwendung von Nullsteuersätzen und Steuerbefreiungen

\begin{tabular}{|c|c|c|c|c|c|c|c|c|c|c|c|c|c|c|c|c|c|}
\hline \multirow{2}{*}{ Land } & \multicolumn{17}{|c|}{ Güterart } \\
\hline & 1 & 2 & 3 & 4 & 5 & 6 & 7 & 8 & 9 & 10 & 11 & 12 & 13 & 14 & 15 & 16 & 17 \\
\hline Belgien & & & B & & B & & B & & & $\mathrm{N}$ & & & & B & & & \\
\hline $\begin{array}{l}\text { Bundesrepublik } \\
\text { Deutschland }\end{array}$ & & & B & & B & & B & & & & B & B & & B & & & \\
\hline Dänemark & & & B & & B & & B & & & $\mathrm{N}$ & & B & & B & & & B \\
\hline Frankreich & & & B & & $\mathrm{B}$ & & B & & & & B & & & B & B & & B \\
\hline Großbritannien & $\mathrm{N}$ & $\mathrm{N}$ & B & $\mathrm{N}$ & B & $\mathrm{N}$ & B & & $\mathrm{N}$ & $\mathrm{N}$ & & & & B & & & \\
\hline Irland & $\mathrm{N}$ & $\mathrm{N}$ & B & $\mathrm{N}$ & B & & B & $\mathrm{N}$ & & & B & & B & B & & $\mathrm{N}$ & \\
\hline Italien & $\mathrm{N}$ & & B & & $\mathrm{B}$ & & B & & & $\mathrm{N}$ & & & & B & & & \\
\hline Luxemburg & & & B & & $\mathrm{B}$ & & B & & & & & & & B & & & \\
\hline Niederlande & & & B & & B & & B & & & $\mathrm{N}$ & & & & B & & & \\
\hline Norwegen & & & B & & & & B & & & & & & & B & & & B \\
\hline Portugal & $\mathrm{N}$ & & B & B & $\mathrm{B}$ & & B & & $\mathrm{N}$ & $\mathrm{N}$ & B & B & & B & & $\mathrm{N}$ & B \\
\hline Spanien & & & B & & B & & B & & & & & B & & B & & B & B \\
\hline Schweden & & & B & $\mathrm{N}$ & $\mathrm{B}$ & & B & & & $\mathrm{N}$ & & & & B & & & B \\
\hline Türkei & $\mathrm{N}$ & & B & & $\mathrm{B}$ & & B & & & & & B & & B & & & \\
\hline Argentinien & B & & B & B & B & & B & & & & & & & B & & B & \\
\hline Chile & B & & B & & $\mathrm{B}$ & & B & & & & B & B & & B & & & \\
\hline Costa Rica & B & & B & B & B & & B & & & & & & & B & & B & \\
\hline
\end{tabular}




\begin{tabular}{|c|c|c|c|c|c|c|c|c|c|c|c|c|c|c|c|c|}
\hline $\begin{array}{l}\text { Kolumbien } \\
\text { Mexiko }\end{array}$ & $\begin{array}{l}\mathrm{B} \\
\mathrm{N}\end{array}$ & $\begin{array}{l}\mathrm{N} \\
\mathrm{B}\end{array}$ & $\begin{array}{l}\text { B } \\
\text { B }\end{array}$ & $\mathrm{B}$ & $\begin{array}{l}\text { B } \\
\text { B }\end{array}$ & B & $\begin{array}{l}\text { B } \\
\text { B }\end{array}$ & $\begin{array}{l}\mathrm{B} \\
\mathrm{B}\end{array}$ & $\begin{array}{l}\mathrm{B} \\
\mathrm{B}\end{array}$ & $\begin{array}{l}\mathrm{B} \\
\mathrm{B}\end{array}$ & B & & B & B & $\mathrm{N}$ & \\
\hline Madagaskar & B & & B & $\mathrm{B}$ & B & B & B & B & B & B & & B & B & & B & \\
\hline Marokko & B & & & & B & & B & B & B & B & B & & & B & $\mathrm{N}$ & \\
\hline Niger & B & & B & $\mathrm{B}$ & B & B & B & B & B & B & B & B & B & B & & \\
\hline Korea & & & B & B & B & & B & B & B & B & B & & B & & & B \\
\hline Philippinen & B & & B & & B & & B & B & & B & B & B & B & & & \\
\hline Taiwan & B & & B & & B & & & & & B & B & B & B & & B & \\
\hline Neuseeland & & & & & & & B & & & & & & & & & \\
\hline
\end{tabular}

$$
\mathrm{B}=\text { Steuerbefreiung }, \quad \mathrm{N}=\text { Nullsteuersatz }
$$

Liste der Güterarten:

$\begin{array}{ll}1 & \text { Nahrungsmittel (unkonserviert) } \\ 2 & \text { Nahrungsmittel (konserviert) } \\ 3 & \text { Medizinische Leistungen } \\ 4 & \text { Arzneimittel } \\ 5 & \text { Leistungen für Bildungszwecke } \\ 6 & \text { Wohnungskauf } \\ 7 & \text { Wohnungsvermietung } \\ 8 & \text { Bekleidung } \\ 9 & \text { Bücher }\end{array}$

$\begin{array}{ll}10 & \text { Zeitungen } \\ 11 & \text { Unterhaltung, Sport } \\ 12 & \text { Museen } \\ 13 & \text { Staatliche Leistungen } \\ 14 & \text { Finanzielle Leistungen } \\ 15 & \text { Gebrauchtwarenhandel } \\ 16 & \text { Landwirtschaftliche Einsatzmittel } \\ 17 & \text { Künstlerische Erzeugnisse }\end{array}$

Quelle: TAIT [1988], S. 52. 
Unter verteilungspolitischen Gesichtspunkten versucht man durch Differenzierung der Steuersätze insbesondere durch Befreiungen die regressive Wirkung der Umsatzsteuer einzudämmen. Mit steigendem Einkommen sinkt der Anteil der Konsumausgaben, hier vor allem der Anteil von Gütern des täglichen Bedarfs wie bspw. Grundnahrungsmittel oder Wohnungsmieten. Haushalte der unteren Einkommensschichten hätten bei einem einheitlichem Steuersatz für alle Güter einen relativ höheren Steuerbeitrag zu leisten als Haushalte der oberen Einkommensschichten ${ }^{36}$.

Beide Rechtfertigungen von Befreiungen und Nullsteuersätzen sind nicht unumstritten. So findet man neben einer grundsätzlichen $\mathrm{Ab}$ lehnung der Musgrave'schen Definition ${ }^{37}$ meritorischer Güter ${ }^{38}$ auch gewisse Abgrenzungsprobleme vor. Unter welchen Umständen weisen bestimmte Güter meritorische Eigenschaften auf und unter welchen nicht? Man denke nur an Sportübertragungen der Fernsehanstalten, um zu erkennen, daß die Übergänge fließend sind. Das Sportereignis als solches mag zwar von kulturellem Interesse sein. Da die Akteure in vielen Fällen jedoch kaum von wandelnden Litfaßsäulen zu unterscheiden sind, treten gewisse Zweifel auf.

Gegen verteilungspolitisch begründete Steuerermäßigungen und befreiungen kann angeführt werden, daß Inzidenzanalysen häufig die Annahme zugrunde liegt, die Umsatzsteuer werde voll auf die Endabnehmer übergewälzt. Geht man dagegen davon aus, daß eine Steuererhöhung zum Teil den Konsumenten, zum Teil aber auch das Unternehmen bzw. die Faktorentlohnung für Arbeit und Kapital „,belastet " ${ }^{39}$, so ist die regressive Wirkung der Steuer auch davon abhängig, in welchem Maß Lohn- und Kapitaleinkommen von der Steuer getroffen werden und wie sich beide Einkommensarten auf die einzelnen Einkommensklassen verteilen.

Das Hauptargument gegen verteilungspolitisch motivierte Steuersatzdifferenzierungen ist $\mathrm{m}$. E. jedoch darin zu sehen, daß eine Steuer-

${ }^{36}$ Für nähere Erläuterungen zu diesem Aspekt siehe bspw. RABOY [1990] oder OECD [1988], S. 122 ff.

${ }^{37}$ Siehe hierzu MUSGrave [1973], S. 80 f.

38 Zum Beispiel MCLURE [1968].

${ }^{39}$ Siehe bspw. HENDERSON/QUANDT [1983], S. $158 \mathrm{f}$. 
befreiung allen Einkommensschichten zugute kommt und der Regressivität eines allgemeinen Steuersatzes wirkungsvoller mit gezielten Transferzahlungen für untere Einkommensklassen zu begegnen wäre als durch Differenzierung der Steuersätze nach Gütergruppen. In einer empirischen Studie kam KitTerer [1982] zu dem Schluß, „daß die dadurch erzielte Entlastung der Armen im Vergleich zu den Reichen absolut gering ist ... (S. 134)“.

Doch selbst wenn man diese Motive akzeptiert, ist weder mit meritorischen noch mit Verteilungsargumenten begründbar, weshalb für Befreiungen kein Vorsteuerabzugsrecht eingeräumt werden sollte. Unter beiden Argumenten scheint diese Maßnahme eher halbherzig oder sogar in sich widersprüchlich zu sein. Auf der einen Seite möchte man bestimmte Güter steuerlich bevorzugen, andererseits doch nicht ganz auf eine Besteuerung verzichten. Was letztendlich dabei herauskommt ist eine unbestimmte Steuerbelastung dieser Güter, die weder einer gezielten Steuerung der Verteilungswirkung noch einer eindeutigen Förderung dient.

Es gibt jedoch eine Reihe von Gründen, die den Vorsteuerausschluß gerechtfertigt erscheinen lassen. Einer der wichtigsten davon ist, daß bestimmte Leistungen - vor allem im finanziellen Bereich - steuerlich schwer zu erfassen sind. Zum Beispiel erweist sich die Bestimmung des steuerbaren Umsatzes von Kreditinstituten, Banken, Versicherungsunternehmen und dgl. als überaus problematische Angelegenheit ${ }^{40}$. Da auf der anderen Seite keines der oben angeführten Argumente gegen eine Besteuerung derartiger Leistungen spricht, begnügt man sich nicht selten mit der Nachholwirkung einer Befreiung.

Darüber hinaus führt die Anwendung von Nullsteuersätzen im Vergleich zu Befreiungen zu einem geringeren Steueraufkommen. Wollte man dasselbe Aufkommen erzielen, müßten die restlichen Steuersätze angehoben werden, was eine stärkere Verzerrung der relativen Konsumentenpreise nach sich zieht. Des weiteren ist mit jeder Differenzierung der Steuersätze - so auch einer Nullbesteuerung - ein höherer Verwaltungsaufwand verbunden. Bei Anwendung eines Nullsteuersatzes müßte u. a. kontrolliert werden, ob die von den relevanten Unternehmen beanspruchten Vorsteuerabzugsbeträge berechtigt sind, ohne daß

${ }^{40}$ Siehe hierzu auch Gillis [1990] und Garber/RABOY [1990]. 
hierdurch (direkt) Steuereinnahmen erzielt werden.

Es erhebt sich jedoch die Frage, ob die Aufrechnung von Aufwand und Ertrag in einem derart eng gesteckten Rahmen überhaupt sinnvoll sein kann. Unter diesem Gesichtspunkt wäre jegliche zerstörende Qualitätskontrolle wie Crashtests oder Belastungsprüfungen ineffizient. Auch anhand der folgenden Überlegung ist dieser Einwand gegen Nullsteuersätze zu relativieren: Produziert ein Unternehmen sowohl besteuerte als auch befreite Güter, dürfen nur diejenigen Vorsteuern abgesetzt werden, die unmittelbar mit der Erzeugung des besteuerten Gutes in Verbindung stehen. Abgesehen davon, daß die Kontrolle der von diesem Unternehmen geltend gemachten Vorsteuerbeträge generell nicht ganz unproblematisch ist, entsteht durch die Befreiung ein höherer Verwaltungsaufwand bei dem Finanzamt und bei dem Unternehmen selbst als durch eine Nullbesteuerung an deren Stelle.

Daneben findet man noch merkwürdigere Argumente wie beispielsweise eines des Rates der Europäischen Gemeinschaft, daß Nullsteuersätze dem Endkonsum zugute kommen sollten; eine Nullbesteuerung typischer Zwischenprodukte wirke dabei zu indirekt ${ }^{41}$. Sofern der Abnehmer des Zwischenprodukts selbst besteuert wird, kann dieses Argument nur bekräftigt werden. Der für das vorgelagerte Unternehmen relevante Nullsteuersatz würde überhaupt keine Wirkung ausüben allerdings jeder andere Steuersatz auch nicht (lediglich eine Befreiung des Zwischenprodukts käme dem Endkonsum „zugute“). Handelt es sich bei dem Abnehmer dagegen um ein befreites Unternehmen, würde der Nullsteuersatz sehr wohl eine Wirkung zeigen. Da die Vorleistung nicht mit Steuern belastet ist, könnte er seine eigenen Produkte billiger anbieten.

Das Argument der problematischen Einbindung von Finanzintermediären in das Umsatzsteuersystem ist nicht so ohne Weiteres von der Hand zu weisen. Frankreichs Steuerregelungen zeigen aber, daß auch der Bankensektor - zumindest teilweise - integriert werden $\mathrm{kann}^{42}$.

${ }^{41}$ Siehe TAIT [1988], S. 54.

${ }^{42}$ Frankreich besteuert die im privaten Sektor ausstehenden Kredite mit einem Steuersatz von 21 Prozent. Die Steuer ist kein Teil der Umsatzsteuer. Eine Bank kann jedoch für die Intergration in das Umsatzsteuersystem optieren. Dann reduziert sich der Steuersatz auf $14 \%$ und geleistete Vorsteuerbeträge 
Ebenso gibt es Überlegungen, wie Versicherungsunternehmen zu besteuern wären ${ }^{43}$. Was die erhöhte Verzerrung einer Substitution von Befreiungen durch Nullsteuersätze bei gleichbleibendem Steueraufkommen betrifft, darf nicht unterschlagen werden, daß auch von Befreiungen verzerrende Wirkungen ausgehen, die noch dazu nicht ohne weiteres abzuschätzen sind. Zudem spielt die Art und Weise, wie die übrigen Steuersätze erhöht werden, keine unbedeutende Rolle in der Einschätzung der allokativen Wirkung des resultierenden Steuersystems.

Wir wollen an dieser Stelle nicht weiter in die Diskussion über die Zweckmäßigkeit eines Ausschlusses des Vorsteuerabzugsrechts einsteigen, sondern begnügen uns mit der Feststellung, daß keiner der bisher angegebenen Gründe rundum überzeugt. Insbesondere bedarf die These einer stärkeren negativen Allokationswirkung von Nullsteuersätzen der genaueren Untersuchung ${ }^{44}$. Solange die von Befreiungen ausgehenden Effizienzwirkungen nicht exakter erfaßbar sind, kann sie nicht überzeugen. Im nächsten Kapitel wird deshalb eine Methode entwickelt, die eine Abschätzung der allokativen Wirkung auf den Endverbrauch erlaubt und damit auch die Anwendung von Erkenntnissen aus der Optimalsteuertheorie ermöglicht.

dürfen abgesetzt werden. Außerdem wird die Umsatzsteuer von der Bank in Rechnung gestellt und kann wiederum von deren Kunden ggf. als Vorsteuer geltend gemacht werden (TAIT [1988], S. 95).

${ }^{43}$ Siehe bspw. Barham/Poddar/Whalley [1987].

${ }^{44} \mathrm{Zwar}$ gibt es empirische Untersuchungen in dieser Richtung - u. a. die von BALlard/Shoven/Whalley [1982] oder BALLARD/ScholZ/ SHOVEN [1987] -, wie wir noch sehen werden, sind sie jedoch nicht ganz unproblematisch. 
Peter Gottfried - 978-3-631-75208-1

Downloaded from PubFactory at 01/11/2019 07:06:43AM

via free access 


\section{Kapitel II}

\section{Effektive Steuersätze}

Die große Attraktivität, die eine Netto-Allphasenumsatzsteuer - zumindest auf finanzwissenschaftliche Kreise - ausübt, ist nicht zuletzt darauf zurückzuführen, daß sich ein auf alle Güter und Leistungen angewandter allgemeiner Steuersatz den meisten ökonomischen Entscheidungen der Unternehmen und privaten Haushalte gegenüber neutral verhält. Steuerberechnungsverfahren und allgemeiner Steuersatz gewährleisten, daß weder die relativen Preise, die die Konsumgüterwahl der Haushalte bestimmen, noch die relativen Preise, an denen die Unternehmen ihre Produktions-, Investitions- und Finanzierungsentscheidungen ausrichten, durch die Steuer verzerrt werden. Bei letzterem spricht man auch von Wettbewerbsneutralität der Steuer ${ }^{45}$.

Diese Neutralitätswirkungen sind allgemein unbestritten. Weniger konform sind dagegen die Ansichten darüber, welche Entscheidungen steuerlich verzerrt werden, wenn man die Steuersätze differenziert oder aber Befreiungen bei gleichzeitigem Ausschluß des Vorsteuerabzugsrechts einführt. Beispielsweise vertritt Davies [1986] die Meinung: „... a uniform VAT on all firms imposes the same proportional tax costs and is neutral toward the choice of production methods or the use of productive resources" (S. 205). In ähnlich pauschaler Weise äußert sich TAIT [1988]: „Multiple rates distort both consumer and producer choices."

Dagegen führt MCLURE [1987] sehr detailliert aus, daß nur Befreiungen, nicht aber differenzierte Steuersätze an sich für allokative Verzerrungen $z$ wischen den Unternehmen verantwortlich sind: „An exempt firm pays no tax on sales but can take no credits on inputs. Yet its customers must pay tax on the full amount of their sales and have no tax on inputs for which to take credit. Because of this break in the chain of credits, the total tax load is actually increased by exemption. This places exempt activities at a disadvantage in both domestic and

${ }^{45} \mathrm{Zu}$ dieser allokationspolitischen Verwendung des Begriffes siehe auch NEUMARK [1970], S. $261 \mathrm{ff}$. 
international markets, distorting resource allocation and reducing international competitiveness. By comparison, zero rating of intermediate stages in the production-distribution process has no effect on ultimate liability. The zero-rated stage pays no tax on sales but is allowed credit for tax paid on purchases, unlike the situation under exemption. Not surprisingly, many observers believe that zero rating is far preferable to exemption from an economic point of view, despite the need to include in the system firms that receive net refunds rather than pay tax, since it avoids the distortions of exemption " $(\mathrm{S} .74 \mathrm{f} \text {. })^{46}$.

Im ersten Abschnitt dieses Kapitels werden wir die Argumentation MCLURE's anhand eines einfachen Allgemeinen Gleichgewichtmodells formal bestätigt finden. Das Modell dient jedoch in erster Linie dem Zweck, aus den nominellen Umsatzsteuersätzen (inklusive Befreiungen) äquivalente Steuersätze zu berechnen. Der Begriff äquivalente Steuersätze ist dabei so zu verstehen, daß sich bei Anwendung dieser Sätze auf bestimmte Endnachfragekategorien dasselbe Gleichgewicht einstellt wie bei einer Umsatzbesteuerung zu den nominellen Steuersätzen. Da wir im weiteren Verlauf ausschließlich eine Umsatzsteuer vom Konsumtyp zugrundelegen, kommen in erster Linie äquivalente Konsumsteuersätze in Betracht. In Teilabschnitt (4.3) und (4.4) wird sich jedoch zeigen, daß eine Besteuerung der Konsumausgaben nicht ausreicht, um das durch die Umsatzsteuer charakterisierte Gleichgewicht zu erreichen.

Das Grundprinzip bei der in Abschnitt (4) erläuterten Berechnung äquivalenter Steuersätze besteht in einem Vergleich der Restriktionen, denen die Wirtschaftssubjekte bei einer Umsatzsteuer unterliegen, mit denjenigen, die sich im steuerlosen Zustand einstellen würden. Die äquivalenten Steuersätze werden dann gerade so gewählt, daß sich unter beiden Steuerregimen dieselben Konsumentenpreise, dieselben Faktorpreise und damit schließlich dieselben Angebots- und Nachfragereaktionen aller Wirtschaftssubjekte ergeben. Dabei unterstellen wir in Teilabschnitt (4.1) zunächst ein stark vereinfachtes Modell mit nur einer Endnachfragekategorie - dem Konsum - und erweitern es im Anschluß an ein kleines numerisches Beispiel (Teilabschnitt (4.2)) auf Außenhandel (Teilabschnitt (4.3)) und Investitionen (Teilabschnitt (4.4)).

${ }^{46}$ Diese Auffassung wird u. a. von CNOSSEN ([1981], S. 58) und POHMER ([1983], S. 389 f.) geteilt. 
Mit Hilfe der äquivalenten Steuersätze ist es nun möglich, die von den nominellen Umsatzsteuersätzen effektiv ausgehenden Wirkungen abzuschätzen. Man bezeichnet sie daher auch einfach als effektive Steuersätze. Dieser Begriff wurde von KAY und WARREN [1980] geprägt. $\mathrm{Da}$ sie bei ihrer Berechnung effektiver Steuersätze jedoch einen Weg einschlugen, der sich in der Methodik und auch im Ergebnis von dem hier präsentierten unterscheidet, wird mit Abschnitt (2) eine kurze Beschreibung ihrer Vorgehensweise der oben skizzierten vorangestellt. Abschnitt (3) kann wiederum als eine Art Vorstufe zu Abschnitt (4) betrachtet werden, denn die Frage nach den effektiven Steuersätzen ist zwangsläufig eng mit der Frage nach der effektiven Steuerlast verbunden. Den Abschluß des Kapitels bildet schließlich die Darstellung der äquivalenten Steuersätze, die sich aus der in Kapitel III erstellten Datenbasis ergeben.

Im unmittelbar anschließenden Abschnitt wird nach einer einführenden Beschreibung des allgemeinen Gleichgewichtmodells die Besteuerungstechnik der Umsatzsteuer formalisiert und ihre eingangs erwähnte Wettbewerbsneutralität hinsichtlich der unternehmerischen Entscheidungen überprüft.

\section{Die formale Darstellung der Umsatzsteuer}

Eine geschlossene Volkswirtschaft bestehe aus $n$ Unternehmen und einem repräsentativen Haushalt ${ }^{47}$. Der Haushalt maximiert seinen Nutzen

$$
U=U\left(C_{1}, \ldots, C_{n}, F\right),
$$

unter der Nebenbedingung: $\quad Y^{m a x}=\sum_{i=1}^{n} p_{i} C_{i}+w F$.

Die zu Konsumentenpreisen $p_{i}(i=1, \ldots, n)$ bewerteten Ausgaben für Konsumgüter $C_{i}$ und die nach dem Opportunitätskostenprinzip mit

${ }^{47}$ Die Annahme eines einzigen repräsentativen Haushalts stellt keine Einschränkung der in diesem Kapitel erzielten Ergebnisse dar. Sie vereinfacht lediglich die Notation. 
dem Lohnsatz $w$ bewerteten Aufwendungen für Freizeit $F$ müssen dem maximalen Einkommen $Y^{\max }$ entsprechen.

Das maximale Einkommen $Y^{\max }=r \bar{K}+w E+T R$ besteht seinerseits aus den Faktoreinkommen für den sich im Besitz des Haushalts befindenden gesamtwirtschaftlichen Kapitalstock $\bar{K}$, für die insgesamt dem Haushalt zur Verfügung stehenden Zeit $E$ sowie den Transferzahlungen $T R$. Hierbei kennzeichnen $r$ und $w$ die Faktorpreise für Kapital und Arbeit, die Transferzahlungen bestehen aus dem vom Staat erzielten Steueraufkommen, das als Pauschbetrag an den Haushalt transferiert wird. Aus der Nutzenmaximierung resultieren die Konsumnachfragefunktionen $C_{i}=C_{i}\left(p_{1}, \ldots, p_{n}, w, Y^{\max }\right)$ und die Arbeitsangebotsfunktion $L=E-F\left(p_{1}, \ldots, p_{n}, w, Y^{\max }\right)$.

Jedes der $n$ Unternehmen produziert jeweils ein Gut. Zur Herstellung einer bestimmten Outputmenge $Q_{i}$ setzt ein Unternehmen $i$ neben den von Unternehmen $j(j=1, \ldots, n)$ erzeugten Vorleistungen $A_{j i}$ die Primärfaktoren Arbeit $L_{i}$ und Kapital $K_{i}$ ein. Wir nehmen an, daß die intermediären Güter in konstanten Einsatzverhältnissen $a_{j i}=A_{j i} / Q_{i}$ - Leontief-Produktionsstruktur - verwendet werden. Damit sind die Produktionsfunktionen durch

$$
Q_{i}=\min \left[\frac{1}{a_{0 i}} f^{i}\left(K_{i}, L_{i}\right), \frac{A_{1 i}}{a_{1 i}}, \ldots, \frac{A_{n i}}{a_{n i}}\right] \quad i=1, \ldots, n
$$

gegeben, wobei $f^{i}\left(K_{i}, L_{i}\right)$ die Wertschöpfungsfunktionen kennzeichnet. Die Unternehmen maximieren ihre Gewinne (bzw. minimieren ihre Produktionskosten), woraus die Faktornachfragefunktionen für Arbeit und Kapital abgeleitet werden können. Aufgrund der unterstellten Produktionstechnologie ist das Faktoreinsatzverhältnis $K_{i} / L_{i}$ nur von den Faktorpreisen abhängig.

Unterstellen wir ferner vollkommene Konkurrenz auf allen Märkten, müssen die Unternehmen - ohne Berücksichtigung der Umsatzsteuer die Budgetbeschränkungen bzw. Null-Profit-Bedingungen

$$
q_{i} Q_{i}=\sum_{j=1}^{n} a_{j i} q_{j} Q_{i}+w L_{i}+r K_{i} \quad i=1, \ldots, n
$$

einhalten, wobei $q_{i}$ den Produzentenpreis für Gut $i$ kennzeichnet. 
Der Output eines Unternehmens $i$ wird zum Teil als Vorleistung $A_{i j}$ für weitere Unternehmen, zum Teil als Konsumgut $C_{i}$ verwendet; von Investitionen und Außenhandelsbeziehungen sei zunächst abgesehen. Da im Gleichgewicht alle Güter- und Faktormärkte geräumt sind, lassen sich die Marktgleichgewichtsbedingungen für die Gütermärkte durch

$$
Q_{i}=\sum_{i=1}^{n} a_{i j} Q_{j}+C_{i} \quad i=1, \ldots, n
$$

beschreiben.

Berücksichtigt man, daß in Gleichung (2) der Eigenverbrauch $A_{i i}$ bzw. $a_{i i} Q_{i}$ nur eine innerbetriebliche Transaktion darstellt ${ }^{48}$, lassen sich die (gleichgewichtigen) Netto-Umsätze der Unternehmen als

$$
\left(1-a_{i i}\right) q_{i} Q_{i}=\sum_{i \neq j}^{n} a_{i j} q_{i} Q_{j}+q_{i} C_{i} \quad i=1, \ldots, n
$$

darstellen.

Wenden wir uns nun einer nach Gütern differenzierenden Umsatzsteuer zu. Ein steuerpflichtiges und zum Abzug der Vorsteuer berechtigtes Unternehmen $i$ berechnet seine Steuerzahllast $T_{i}^{u}$ nach der Formel:

$$
T_{i}^{u}=\underbrace{\tau_{i}^{u}\left(1-a_{i i}\right) q_{i} Q_{i}}_{\text {Basis-Umsatzsteuer }}-\underbrace{\sum_{j \neq i} \tau_{j}^{u} a_{j i} q_{j} Q_{i} .}_{\text {Vorsteuer }}
$$

Der für das Unternehmen relevante Steuersatz $\tau_{i}^{u}$, angewendet auf die Netto-Umsätze aus Verkauf an unternehmensfremde Dritte, ergibt die Basis-Umsatzsteuerschuld, wovon die auf den Kauf von Vorleistungen angefallenen Vorsteuern zum Abzug gebracht werden. Wäre Unternehmen $i$ darüber hinaus steuerbefreit $\left(\tau_{i}^{u}=0\right)$ - man spricht von einer

48 Der Eigenverbrauch ist in diesem Zusammenhang der unternehmensbezogene Verbrauch selbstproduzierter Güter. Er sollte nicht mit der Privatentnahme des Unternehmers verwechselt werden. Letzterer wird als Teil der Konsumgüter $C_{i}$ erfaßt. 
echten Steuerbefreiung oder einem Nullsteuersatz -, entfiele der erste Term der rechten Seite von Gleichung (4); das Unternehmen erhielte eine Steuerrückerstattung in Höhe der gezahlten Vorsteuern.

Besteht generelle Vorsteuerabzugsberechtigung für alle Unternehmen der Volkswirtschaft, so beläuft sich das gesamtwirtschaftliche Umsatzsteueraufkommen $T^{u}$ - als Summe der einzelwirtschaftlichen Steuerzahllasten - auf

$$
\begin{aligned}
T^{u}=\sum_{i} T_{i}^{u} & =\sum_{i} \tau_{i}^{u}\left(1-a_{i i}\right) q_{i} Q_{i}-\sum_{i} \sum_{j \neq i} \tau_{j}^{u} a_{j i} q_{j} Q_{i} \\
& =\sum_{i} \tau_{i}^{u} q_{i}\left(Q_{i}-\sum_{j} a_{i j} Q_{j}\right) .
\end{aligned}
$$

Unter Verwendung von Gleichung (2) ergibt sich daraus

$$
T^{u}=\sum_{i} \tau_{i}^{u} q_{i} C_{i}
$$

Die Netto-Allphasenumsatzsteuer wirkt somit bei generellem Vorsteuerabzugsrecht wie eine Konsumsteuer. Die Umsatzsteuersätze könnten ebenso gut als Konsumsteuersätze aufgefaßt werden.

Aufgrund dieser Wirkung erscheint es auch plausibel, daß die Umsatzsteuer nicht unmittelbar die Preisbildung der Unternehmen beeinflußt, sondern lediglich einen Keil zwischen Produzenten- und Konsumentenpreis treibt ${ }^{49}$. Wir können diese Vermutung über Gleichungssystem (1) bestätigen. Es spezifiziert, wie erwähnt, die Null-ProfitBedingungen der Unternehmen ohne Berücksichtigung der Steuer.

Nimmt man die Umsatzsteuer in die Null-Profit-Bedingungen auf, dann sind alle Markttransaktionen - die Netto-Umsätze der Unternehmen $\left(1-a_{i i}\right) q_{i} Q_{i}$ sowie deren Vorleistungsbezüge $\sum_{j \neq i} a_{j i} q_{j} Q_{i}-$ nun

49 Unmittelbare Beeinflussung ist dabei so zu verstehen, daß die allokative Verzerrung ausschließlich von der Diskrepanz zwischen Produzenten- und Konsumentenpreis ausgeht. Durch die steuerbedingte Veränderung der relativen Konsumentenpreise werden Substitutionseffekte seitens der Haushalte ausgelöst, die via Markträumungsbedingungen auf den Produktionssektor rückwirken und damit wiederum Einfluß auf die Produktionsentscheidung und die Produzentenpreise ausüben, usw. . Diese jedem Gleichgewichtsmodell inhärenten indirekten oder Second Round Effekte sind für die momentane Überlegung jedoch nicht entscheidend. 
mit Bruttopreisen $p_{i}$ zu bewerten und die an den Fiskus zu entrichtenden Umsatzsteuerbeträge $T_{i}^{u}$ einzubeziehen. Man erhält

$$
\left(1-a_{i i}\right) p_{i} Q_{i}=T_{i}^{u}+\sum_{j \neq i} a_{j i} p_{j} Q_{i}+w L_{i}+r K_{i}
$$

Setzten wir in Gleichung (6) die Berechnungsformel für die Steuerzahllast (4) ein und berücksichtigen ferner den Zusammenhang zwischen Brutto- und Nettopreisen $p_{i}=\left(1+\tau_{i}^{u}\right) q_{i}$, stellt sich nach einigen elementaren Umformungen über

$$
\begin{aligned}
\left(1-a_{i i}\right)\left(p_{i}-\tau_{i}^{u} q_{i}\right) Q_{i} & =\sum_{j \neq i} a_{j i}\left(p_{j}-\tau_{j}^{u} q_{j}\right) Q_{i}+w L_{i}+r K_{i} \\
q_{i} Q_{i} & =\sum_{j=1}^{n} a_{j i} q_{j} Q_{i}+w L_{i}+r K_{i}
\end{aligned}
$$

die ursprüngliche Gleichung (1) wieder ein. Solange generelles Vorsteuerabzugsrecht besteht, hat die Umsatzsteuer keine direkten Auswirkung auf die Null-Profit-Bedingungen der Unternehmen. Daraus folgt, daß die Gültigkeit der zu Beginn des Kapitels beschriebenen Eigenschaft der Wettbewerbsneutralität einer Netto-Umsatzsteuer nicht von der Höhe der Steuersätze, insbesondere von der Erhebung eines allgemeinen Steuersatzes abhängt.

Unter diesem Gesichtspunkt erscheint dann auch die von BALLARD/ Shoven [1987], Ballard/Scholz/Shoven [1978] oder KehoE et al. [1988] gewählte Vorgehensweise gerechtfertigt zu sein: Im Rahmen von Effizienzuntersuchungen verschiedener Umsatzsteuersysteme modellierten sie anstelle der Umsatzsteuer eine einfache Konsumsteuer und interpretierten die Konsumsteuersätze als die entsprechenden Umsatzsteuersätze.

Sobald jedoch einem einzigen steuerbefreiten Unternehmen der Abzug der Vorsteuer versagt bleibt, geht die Wettbewerbsneutralität verloren. In der Gleichung (6) entsprechenden Null-Profit-Bedingung eines steuerbefreiten Unternehmens $h$ wäre $T_{h}^{u}$ gleich Null und wegen $\tau_{h}^{u}=0$ müßte das Unternehmen seiner Produzentenpreis-Kalkulation Bruttopreise für Vorleistungsbezüge zugrundelegen. Die Umsatzsteuersätze 
treiben in diesem Fall nicht nur einen Keil zwischen Produzenten- und Konsumentenpreis, sondern verzerren bereits die relativen Produzentenpreise. Außerdem zeigt die Aufsummierung der einzelwirtschaftlichen Steuerzahllasten wegen

$$
\begin{aligned}
\sum_{i=1}^{n} T_{i}^{u} & =\sum_{i} \tau_{i}^{u} q_{i} Q_{i}-\sum_{i \neq h} \sum_{j=1}^{n} \tau_{j}^{u} a_{j i} q_{j} Q_{i} \\
& =\sum_{i} \tau_{i}^{u} q_{i} Q_{i}-\sum_{i=1}^{n} \sum_{j=1}^{n} \tau_{j}^{u} a_{j i} q_{j} Q_{i}+\sum_{j=1}^{n} \tau_{j}^{u} a_{j h} q_{j} Q_{h} \\
& =\sum_{i=1}^{n} \tau_{i}^{u} q_{i} C_{i}+\sum_{j=1}^{n} \tau_{j}^{u} a_{j h} q_{j} Q_{h},
\end{aligned}
$$

daß auch die direkte Äquivalenz zwischen Umsatz- und Konsumsteuer nicht mehr gewahrt ist. Wie der zweite Summand auf der rechten Seite von Gleichung (7) zeigt, wird nun ein Teil des gesamtwirtschaftlich erzielten Umsatzsteueraufkommens über die Besteuerung von Vorleistungen gewonnen. Dieser Teil müßte gemeinsam mit den nominellen Umsatzsteuersätzen in einer geeigneten Form zu effektiven Steuersätzen verrechnet werden, damit die Umsatzsteuer weiterhin als Konsumsteuer modelliert werden kann.

\section{Effektive Wertschöpfungsteuersätze}

Als erster Schritt in diese Richtung sei die von KaY und WARREN [1980] entwickelte Methode zur Ermittlung effektiver Umsatzsteuersätze vorgestellt. Ihr Ausgangspunkt bildet die Berechnungsformel für die Umsatzsteuerzahllast der Unternehmen. Zieht man in Gleichung (4) den Term $\tau_{i}^{u} a_{i i} q_{i} Q_{i}$ in die Summe hinein, ergibt sich nach Ausklammern von $q_{i} Q_{i}$,

$$
T_{i}^{u}=\left(\tau_{i}^{u}-\sum_{j} \tau_{j}^{u} a_{j i} \frac{q_{j}}{q_{i}}\right) q_{i} Q_{i}
$$




$$
\begin{aligned}
& =\frac{\tau_{i}^{u}-\sum_{j} \tau_{j}^{u} a_{j i} \frac{q_{j}}{q_{i}}}{1-\sum_{j} a_{j i} \frac{q_{j}}{q_{i}}}\left(q_{i} Q_{i}-\sum_{j} a_{j i} q_{j} Q_{i}\right) \\
& =\frac{\tau_{i}^{u}-\sum_{j} \tau_{j}^{u} a_{j i} \frac{q_{j}}{q_{i}}}{1-\sum_{j} a_{j i} \frac{q_{j}}{q_{i}}}\left(w L_{i}+r K_{i}\right),
\end{aligned}
$$

wobei für den letzten Übergang Gleichung (1) verwendet wurde. Aus dieser Darstellung ist erkennbar, in welcher Beziehung die Umsatzsteuersätze zur Wertschöpfung des Unternehmens stehen. Definiert man den Wertschöpfungsteuersatz $\tau_{i}^{w}$ als

$$
\tau_{i}^{w}:=\frac{\tau_{i}^{u}-\sum_{j} \tau_{j}^{u} a_{j i} \frac{q_{j}}{q_{i}}}{1-\sum_{j} a_{j i} \frac{q_{j}}{q_{i}}},
$$

kann das Umsatzsteuersystem in ein äquivalentes Wertschöpfungsteuersystem umgerechnet werden. Das heißt, würde man anstelle der Umsatzsteuer mit den Steuersätzen $\tau_{i}^{u}(i=1, \ldots, n)$ eine Wertschöpfungsteuer mit den Sätzen $\tau_{i}^{w}$ erheben, so erhielte man eine identische Güter- und Faktorallokation unter beiden Besteuerungsalternativen. Die Äquivalenz ist leicht einzusehen, wenn man bedenkt, daß die Wertschöpfungsteuersätze per Konstruktion zu denselben Steuerzahllasten und denselben Konsumentenpreisen führen wie die Umsatzsteuersätze, und darüber hinaus im Fall der Wertschöpfungsteuer Produzenten- und Konsumentenpreise zusammenfallen.

Diese Wertschöpfungsteuersätze bezeichnen KAY/WARREN als „Effective Rates of VAT“. Ins Auge fällt die strukturelle Analogie von Definition (9) zur Berechnungsformel der effektiven Protektion in der Außenhandelstheorie ${ }^{50}$. Darüber hinaus ist unmittelbar zu erkennen, $\mathrm{da} \beta$ eine allgemeine Umsatzsteuer $\left(\tau_{i}^{u}=\tau^{u} \quad \forall i\right)$ nicht nur zu einer allgemeinen Konsumsteuer, sondern ebenfalls zu einer allgemeinen Wertschöpfungsteuer (mit $\tau^{u}=\tau^{c}=\tau^{w}$ ) äquivalent ist.

50 Zur Diskussion der „effective rate of protection“ siehe u. a. JOHNSON [1965], BALASSA [1965], CORDEN [1966]. 
Werden hingegen differenzierte Umsatzsteuersätze angewendet, kann bei relativ kleinen $\tau_{i}^{u}$ der hierzu korrespondierende Wertschöpfungsteuersatz $\tau_{i}^{w}$ negative Werte annehmen. In der Tat entspricht dem Umsatzsteuersystem Großbritanniens, für das eine empirische Berechnung effektiver Steuersätze durchgeführt wurde, eine Wertschöpfungsteuer, die teils besteuert, teils aber auch subventioniert. Da in Großbritannien unter anderem auch Nullsteuersätze zur Anwendung kommen, belief sich bspw. der effektive Wertschöpfungsteuersatz für Nahrungsmittel auf $-24.3 \%$, während der für das Gastronomiegewerbe relevante $17.0 \%$ betrug ${ }^{51}$.

Gerade dieser Punkt läßt es jedoch fraglich erscheinen, ob die effektiven Wertschöpfungsteuersätze zur Interpretation der von der Steuer ausgehenden allokativen Verzerrungen tatsächlich besser geeignet sind als die Betrachtung der nominalen Umsatzsteuersätze an sich. $\mathrm{Zu}-$ mindest ist sie komplizierter, da die steuerbedingte Verzerrung nun in den Produktionsbereich übertragen wurde, wofür es weitaus weniger theoretische Erkenntnisse zur effizienten Besteuerung (bei gleichzeitiger Berücksichtigung der Produktionsverflechtung) gibt ${ }^{52}$. Um die Steuersätze mit dem Konsumentenverhalten in Verbindung zu bringen, argumentieren KAY und WARREN: „It is this pattern of effective rates which determines the extent to which the tax distorts economic activity. The high effective tax rate on catering encourages entertainment at home while the negative rate on food manufacture discourages home baking ..." (S. 1 f.).

Sie vergleichen somit die industrielle Fertigung der Güter mit ähnlich gelagerten Aktivitäten der Haushalte. Denkt man an die Umsatzsteuer, die den ermittelten Wertschöpfungsteuersätzen zugrundeliegt, ist diese Interpretation durchaus einleuchtend. Während ein Unternehmen die gezahlte Vorsteuer absetzen kann, muß der Haushalt die Steuern auf alle benötigten Ingredienzen selbst tragen. Zwar entfiele durch die „Produktion zu Hause" die Umsatzsteuer auf das Endprodukt, der negative Wertschöpfungsteuersatz indiziert jedoch, daß die bereits geleisteten Steuerzahlungen diese Einsparung überwiegen.

Dennoch erscheint die Interpretation etwas künstlich, zumal sie ein

${ }^{51}$ Siehe KaY / Hemming [1981], Tabelle 2, S. 81.

${ }^{52}$ Als Ausnahme wäre bspw. BHATIA $[1981,1986]$ zu nennen. 
sehr spezielles Verhalten der privaten Haushalte unterstellt und darüber hinaus identische Produktionstechnologien für Unternehmen und Haushalte voraussetzt. Wie sollte zum Beispiel nach dieser Argumentationslinie das Nachfrageverhalten für diejenigen Güter beurteilt werden, die nicht in Heimarbeit produzierbar sind? Stützt man sich hier auf die "traditionelle“ Betrachtungsweise, daß die Güternachfrage durch die Konsumentenpreise und das (verfügbare) Haushaltseinkommen determiniert wird, ist eine zusätzliche Überlegung - nämlich, wie sich die Wertschöpfungsteuersätze auf die Konsumentenpreise auswirken erforderlich. Eine nicht ganz einfache Überlegung obendrein, denn aufgrund der Produktionsverflechtung bilden die Wertschöpfungsteuersätze ein interdependentes System von sich gegenseitig verstärkenden oder kompensierenden Preiseffekten. Einem einzelnen Wertschöpfungsteuersatz $\tau_{i}^{w}$ - isoliert betrachtet - ist nicht anzusehen, ob der resultierende Konsumentenpreis $p_{i}$ niedriger, gleich oder höher ist als der entsprechende Preis im steuerlosen Zustand.

Treffen wir die Annahme, alle Unternehmen - insbesondere auch die steuerbefreiten - seien vorsteuerabzugsberechtigt. Aus dem vorangehenden Abschnitt ist bekannt, daß dann lediglich der private Konsum mit den entsprechenden Umsatzsteuersätzen behaftet ist. Eine Betrachtung der effektiven Wertschöpfungsteuersätze führt nicht zu dieser Erkenntnis; sie ließe eher das Gegenteil vermuten. Es ist nicht zu erkennen, daß die für befreite Unternehmen errechneten negativen Wertschöpfungsteuersätze gerade die Preissteigerung kompensieren, die durch die steuerbedingte Verteuerung der Vorprodukte ausgelöst wurde. Damit läßt sich aber die allokative Verzerrung viel einfacher über die Beeinflussung der relativen Konsumentenpreise durch die Umsatzsteuersätze erklären als über die äquivalente Besteuerung der Wertschöpfung. Letztere ist der ökonomischen Interpretation - zumindest in diesem Fall - viel weniger zugänglich.

Lassen wir dagegen die Annahme der generellen Vorsteuerabzugsberechtigung fallen, so stimmen Umsatzsteuer- und äquivalente Konsumsteuersätze nicht mehr überein. Die effektive Steuerbelastung des Konsums entspricht nicht mehr den in Gleichung (5) berechneten Konsumsteuerbeträgen, da nun sowohl Produzenten- als auch Konsumentenpreise verzerrt werden. Vor allem zeigt der für befreite Unter- 
nehmen relevante Umsatzsteuersatz von Null die Nachholwirkung der Steuer nicht an. Da die Herleitung der Wertschöpfungsteuersätze auf der Berechnungsformel für die Steuerzahllast der Unternehmen basiert, ist leicht einzusehen, daß der entsprechende Wertschöpfungsteuersatz ebenfalls gleich Null ist. Die effektive Wirkung eines Ausschlusses von der Vorsteuerabzugsberechtigung ist folglich mit der Berechnung eines äquivalenten Wertschöpfungsteuersystems ebensowenig erfaßbar wie mit den nominellen Umsatzsteuersätzen an sich. Entgegen der mitunter vertretenen Meinung, daß die effektiven Wertschöpfungsteuersätze die ,versteckte Besteuerung“ aufdecken ${ }^{53}$, erklärt sich die zum Teil starke Streuung der Sätze allein aus der veränderten Bezugsbasis bzw. Bemessungsgrundlage.

Da vor allem im englischsprachigen Raum die Umsatzsteuer häufig als reine Variante einer Wertschöpfungsteuer angesehen wird, mag die Ermittlung effektiver Wertschöpfungsteuersätze gerechtfertigt und unter diesem Gesichtspunkt auch informativ sein, zumal sie deutlich vor Augen führt, daß gerade die am häufigsten praktizierte NettoAllphasenumsatzsteuer (berechnet nach dem Vorsteuerabzugsverfahren) keine so enge Verwandtschaft zur Wertschöpfungsteuer erkennen läßt. Für andere Typen der Netto-Umsatzsteuer hingegen ist diese Verwandtschaft durchaus zutreffend. Die effektiven Wertschöpfungsteuersätze geben beispielsweise an, wie äquivalente Umsatzsteuersätze in unserem Modell zu wählen wären, wenn man - aus welchen Gründen auch immer - vom Vorsteuerabzugsverfahren auf das Vorumsatzabzugsverfahren umstellen möchte, ohne dadurch eine Veränderung der Güterallokation zu bewirken. Für unsere Zielsetzung erscheint die Betrachtung effektiver Wertschöpfungsteuersätze jedoch weniger geeignet.

53 "They occur because traders are unable to claim full credit for some inputs, yet the VAT is applied to the full value of their sale", TAIT [1988], S. 44. 


\section{Der Steuergehalt der Endnachfrage}

Wie in den vorangehenden Abschnitten bereits mehrfach angedeutet wurde, beeinflussen die Umsatzsteuersätze sowohl Produzenten- als auch Konsumentenpreise, falls einige der Unternehmen steuerbefreit und nicht zum Abzug der Vorsteuer berechtigt sind. Die Frage nach der effektiven Belastung des Konsums trägt somit den Charakter einer Inzidenz. Aus der umfangreichen Literaturliste von Inzidenzanalysen ${ }^{54}$ wollen wir deshalb exemplarisch zwei empirische Arbeiten - die eine von KitTerer [1978], die zweite von SKolka [1987] - über die Inzidenz indirekter Steuern ${ }^{55}$ herausgreifen und etwas näher betrachten. Zwar sind mit derartigen Inzidenzanalysen üblicherweise eher verteilungspolitische als effizienzorientierte Zielsetzungen verbunden - etwa die Frage ob indirekte Steuern progressiv oder regressiv wirken -, die angewandte Methodik läßt jedoch auch eine enge Verbindung zu unserer Fragestellung erkennen.

Zum einen verwenden beide ein Input-Output-Modell für ihre Analyse, zum zweiten kommt in beiden Arbeiten das von U. HICKs [1946] eingeführte Konzept der formalen Inzidenz bei der Zurechnung der Umsatzsteuer auf die einzelnen Kategorien der Endnachfrage zur Anwendung. Es besagt, daß am Ende einer Wirtschaftperiode die Umsatzsteuerzahllasten von den Unternehmen an den Fiskus entrichtet werden mußten und damit auch in dem Preis enthalten sind, den die Konsumenten zu zahlen bereit waren ${ }^{56}$.

Im Detail weichen sie, sowohl in ihrer Auffassung über die Zurechenbarkeit bestimmter indirekter Steuerarten, als auch in der konkreten

54 Bedau/Göseke [1972], Browning [1985], Dennerlein [1982], KaISER [1989], Musgrave et al. [1974], Pechman/OKner [1974] oder PECHMAN [1985], um nur einige zu nennen.

55 Nach der Definition des Statistischen Bundesamtes versteht man unter indirekten Steuern diejenigen Steuerarten, die bei Unternehmen erhoben und im Zuge der Gewinnermittlung absetzbar sind (STBA [1989], S. 79). Darunter fallen auch die Umsatzsteuern.

${ }^{56}$ Dagegen wird der Begriff häufig auch als die vom Gesetzgeber intendierten Steuerträger verstanden (vgl. u. a. STIGLITZ/SCHÖNFELDER [1989], S. 148 und BRÜMMERHOFF [1988], S. 421). 
methodischen Vorgehensweise voneinander ab. Die Unterschiede basieren zum Teil auf verschiedenen ökonomischen Annahmen wie, bspw. hinsichtlich der Überwälzbarkeit der Gewerbesteuer auf die Konsumentenpreise oder bezüglich der Behandlung von Importen, zum Teil sind sie auf unterschiedliche Ausweistechniken in den von ihnen verwendeten Statistiken zurückzuführen. Diese spezifischen Unterschiede sind in unserem Zusammenhang jedoch nicht von Interesse. Wir betrachten hier ausschließlich die ebenfalls differierende Methode der Zurechnung der Umsatzsteuer auf die Komponenten der Endnachfrage, wobei letztere im Rahmen unseres vereinfachten Modells - nur aus Konsumausgaben bestehen.

Die Grundgleichung eines Input-Modells kann aus den Markträumungsbedingungen (2) für die Gütermärkte - mit den entsprechenden Produzentenpreisen multipliziert - gewonnen werden. In Matrixschreibweise erhalten wir

$$
\begin{aligned}
\hat{q} Q & =V \hat{q} Q+\hat{q} C \\
& =(I-V)^{-1} \hat{q} C,
\end{aligned}
$$

wobei $I$ die $n$-dimensionale Einheitsmatrix, $\hat{q}$ die Diagonalmatrix der Produzentenpreise mit den Elementen $q_{i}(i=1, \ldots, n)$ auf der Hauptdiagonalen und allen anderen Elementen gleich Null und $V$ die Matrix der mit Produzentenpreisen bewerteten Input-Koeffizienten kennzeichnet; ein typisches Element der Matrix $V$ ist bspw. $v_{i j}=a_{i j} \frac{q_{i}}{q_{j}}$. $Q$ und $C$ repräsentieren die $n$-dimensionalen Spaltenvektoren für den Unternehmensoutput bzw. die Konsumgüter.

Betrachten wir zunächst das von KitTerer gewählte Vorgehen. Er definiert die Steuerkoeffizienten $t_{i}^{k}:=T_{i}^{u} /\left(q_{i} Q_{i}\right)$ als das Verhältnis zwischen der Umsatzsteuerzahllast $T_{i}^{u}$ eines Unternehmens und dessen Bruttoproduktionswert. Das gesamtwirtschaftliche Umsatzsteueraufkommen $T^{u}$ ergibt sich nach dieser Definition trivialerweise als Produkt des Zeilenvektors der Steuerkoeffizienten $t^{k^{\prime}}$ und dem Spaltenvektor der Bruttoproduktionswerte

$$
T^{u}=t^{k^{\prime}} \hat{q} Q
$$


woraus nach Einsetzen von (10) die Gleichung

$$
T^{u}=t^{k^{\prime}}(I-V)^{-1} \hat{q} C
$$

folgt. Ersetzt man nun auf der rechten Seite der obigen Gleichung den Konsumgüter-Spaltenvektor $C$ durch die entsprechende Diagonalmatrix $\hat{C}$, so gibt der daraus resultierende Zeilenvektor $\vec{T}^{\prime}{ }_{57}$ mit

$$
\vec{T}^{\prime}=t^{k^{\prime}}(I-V)^{-1} \hat{q} \hat{C}
$$

an, wie sich die Umsatzsteuerbeträge auf die einzelnen Konsumgüterausgaben verteilen. Das $i$-te Element von $\vec{T}^{\prime}$ enthält den Steuerbetrag $T_{i}^{C}$, mit dem die private Endnachfrage nach Gut $i$ effektiv belastet ist.

SKolka hingegen verfährt in drei Schritten. Zunächst setzt er die in der Input-Output-Tabelle unterhalb der Zentralmatrix ausgewiesenen nichtabzugsfähigen Umsatzsteuerbeträge $N U_{i}^{u}$ der Unternehmen ins Verhältnis zu deren Bruttoproduktionswerten. Diese Steuerkoeffizienten $t_{i}^{s}:=N U_{i}^{u} /\left(q_{i} Q_{i}\right)$ werden im Anschluß daran - analog zur Vorgehensweise von KITTERER - mit der Leontief-Inversen und den Netto-Ausgaben für Konsum multipliziert. Aus

$$
{\overrightarrow{T_{n u}^{C}}}^{\prime}=t^{s \prime}(I-V)^{-1} \hat{q} \hat{C}
$$

ist diejenige Konsumbelastung zu ersehen, die sich aufgrund der von den Unternehmen zu tragenden Umsatzsteuer ergibt. Addiert man schließlich die nach Gütern aufgelisteten Steuerbeträge, die der Haushalt direkt zahlt, zu dem Zeilenvektor ${\overrightarrow{T_{n u}^{C}}}^{\prime}$ hinzu, kann durch

$$
{\overrightarrow{T^{C}}}^{\prime}={\overrightarrow{T_{n u}^{C}}}^{\prime}+{\overrightarrow{\tau^{u}}}^{\prime} \hat{q} \hat{C}
$$

die gesamte effektive Steuerbelastung der privaten Endnachfrage ermittelt werden.

${ }^{57}$ Falls die Nomenklatur keine eindeutige Unterscheidung von Skalaren und Vektoren zuläßt, werden Vektoren durch Pfeile gekennzeichnet. 
Auf den ersten Blick erscheinen beide Verfahren recht unterschiedlich; genauer betrachtet, führen sie jedoch zum gleichen Resultat. Eine kurze Überlegung soll dies verdeutlichen. Wir gehen hierzu vom Zeilenvektor der Steuerzahllasten $\vec{T}^{\prime}$ aus. Für steuerbefreite und nicht vorsteuerabzugsberechtigte Unternehmen ist die Steuerzahllast offensichtlich gleich Null. Man könnte nun $\vec{T}^{\prime}$ in einen fiktiven Vektor ${\overrightarrow{T_{a}^{\prime}}}^{\prime}$, der die Steuerzahllast bei Abzugsberechtigung aller Unternehmen beschreibt, und den Vektor $N U^{u \prime}$, der - wie gesehen - die auf den Unternehmen lastende nichtabzugsfähige Umsatzsteuer ausweist, aufteilen. Da sich ${\overrightarrow{T_{a}^{\prime}}}^{\prime}$ von der tatsächlichen Zahllast nur dadurch unterscheidet, daß anstatt der für befreite Unternehmen relevanten Steuerschuld von Null nun negative Beträge in Höhe der gezahlten Vorsteuern stehen, ergibt die Summe von ${\overrightarrow{T_{a}^{\prime}}}^{\prime}$ und $N U^{u^{\prime}}$ die tatsächliche Steuerschuld. Es gilt:

$$
{\overrightarrow{T^{u}}}^{\prime}={\overrightarrow{T_{a}^{\prime}}}^{\prime}+N U^{u^{\prime}}
$$

Nach Division durch die Bruttoproduktionswerte folgt daraus wiederum

$$
t^{k^{\prime}}=t^{a \prime}+t^{s \prime}
$$

Die fiktiven Beträge ${\overrightarrow{T_{a}^{\prime}}}^{\prime}$ lassen sich aus der allgemeinen Berechnungsformel für die Steuerzahllasten - Gleichung (4) - ermitteln. In Matrixform erhält man

$$
{\overrightarrow{T_{a}^{\prime}}}^{\prime}={\overrightarrow{\tau^{u}}}^{\prime}(I-V) \hat{q} \hat{Q},
$$

in Form von Steuerkoeffizienten

$$
t^{a \prime}={\overrightarrow{\tau^{u}}}^{\prime}(I-V) \text {. }
$$

Ersetzen wir nun die Steuerkoeffizienten $t^{k^{\prime}}$ in Gleichung (11) durch unsere aufgeteilten Koeffizienten(12), kann die Vorgehensweise von KitTERER über

$$
\begin{aligned}
\overrightarrow{T^{C}} & =\left(t^{a \prime}+t^{s \prime}\right)(I-V)^{-1} \hat{q} \hat{C} \\
& ={\overrightarrow{\tau^{u}}}^{\prime}(I-V)(I-V)^{-1} \hat{q} \hat{C}+t^{s \prime}(I-V)^{-1} \hat{q} \hat{C} \\
& ={\overrightarrow{\tau^{u}}}^{\prime} \hat{q} \hat{C}+t^{s \prime}(I-V)^{-1} \hat{q} \hat{C}
\end{aligned}
$$


in diejenige von Skolka überführt werden.

Diese Kongruenz gilt allerdings nur in unserem vereinfachten Modell einer geschlossenen Volkswirtschaft ohne Berücksichtigung von Investitionen. Tatsächlich bildet SKoLKA die Input-Koeffizientenmatrix $V$ ausschließlich über die inländische Produktionsverflechtung, während KITTERER seinen Berechnungen eine Koeffizientenmatrix der inländischen Produktion einschließlich der Importe zugrundelegt. Demzufolge betrachtet KITTERER Importgüter als vollkommene Substitute zu vergleichbaren inländischen Produkten, SKoLKA dagegen unterstellt die Armington-Annahme, wonach inländische Güter und Importe, obwohl sie demselben Zweck dienen, aufgrund spezifischer, unter Umständen subjektiver Eigenschaften, verschiedenartige Güter darstellen ${ }^{58}$. In der Regel weichen damit auch die Importgüterpreise von den entsprechenden Preisen inländischer Güter ab, und eine steuerbedingte Preiserhöhung wird nur durch die inländische Produktionsverflechtung an den inländischen Endverbrauch übertragen. Wir werden diesen Punkt an späterer Stelle nochmals aufgreifen.

\section{Die Berechnung effektiver Steuersätze}

Aus den im letzten Abschnitt ermittelten Konsumsteuerbeträgen $\overrightarrow{T^{C}}$ ist der effektive Steueranteil an den Konsumausgaben zu ersehen. Dennoch erscheint dieses Ergebnis für unsere Zielsetzung in gewisser Hinsicht unbefriedigend, denn aus den Steuerbeträgen können nicht ohne weiteres effektive Steuersätze abgeleitet werden. Bemessungsgrundlage für eine äquivalente Konsumsteuer wären die Netto-Konsumausgaben. $\mathrm{Da}$ die äquivalente Konsumsteuer dasselbe Gleichgewicht, insbesondere dieselbe Güterallokation und dieselben Steuerbeträge wie das Umsatzsteuersystem erzeugen soll, wären nur die Konsummengen, nicht aber die Netto-Ausgaben unter beiden Steuerregimen identisch. Die Konsumsteuerbeträge müßten sich über $T_{i}^{C}=\tau_{i}^{c} \tilde{q}_{i} C_{i}$ ergeben, wovon we$\operatorname{der} \tau_{i}^{c}$ noch $\tilde{q}_{i}$ bekannt sind ${ }^{59}$. Davon abgesehen, ist der angewand-

${ }^{58}$ Vgl. hierzu Armington [1969], S. $156 \mathrm{ff}$.

${ }^{59}$ Eine Begründung, warum $q_{i}$ nicht mit $\tilde{q}_{i}$ übereinstimmen kann, wird im nächsten Teilabschnitt gegeben. 
ten Methodik allein nicht zwingend zu entnehmen, ob eine zum gleichen Steueraufkommen führende Konsumsteuer auch tatsächlich dasselbe Gleichgewicht wie die Umsatzsteuer hervorruft. Zur Berechnung der effektiven Konsumsteuersätze wird deshalb der in GotTFriEd/ WIEGARD [1990] beschriebene Weg gewählt.

\subsection{Effektive Konsumsteuersätze}

Sind alle $n$ Unternehmen der Volkswirtschaft zum Abzug der Vorsteuer berechtigt, werden die Produzentenpreise bekanntlich über die NullProfit-Bedingungen (1) bestimmt. Dividiert man diese Gleichungen durch den jeweiligen Unternehmensoutput $Q_{i}$ erhält man

$$
q_{i}=\sum_{j=1}^{n} a_{j i} q_{j}+w l_{i}+r k_{i} \quad i=1, \ldots, n,
$$

wobei $l_{i}=L_{i} / Q_{i}$ und $k_{i}=K_{i} / Q_{i}$ die Faktoreinsatzmengen pro Einheit Output kennzeichnen. Dieses lineare Gleichungssystem kann in Matrixform sehr bequem durch

$$
q=A^{\prime} q+W_{e}
$$

mit $A^{\prime}$ als der transponierten Matrix der Input-Koeffizienten und $W_{e}$ dem Spaltenvektor der Faktorkosten pro Einheit Output beschrieben werden. Aufgelöst nach $q$ ergibt sich daraus

$$
q=\left(I-A^{\prime}\right)^{-1} W_{e} .
$$

Die Produzentenpreise sind somit allein durch die Faktorkosten pro Einheit Output determiniert.

Eine andere Situation finden wir vor, falls $m$ der $n$ Unternehmen steuerbefreit und vom Abzug der Vorsteuer ausgeschlossen sind. Da, ausgehend von Gleichung (6), die anzuwendenden Umsatzsteuersätze $\tau_{h}^{u}$ (mit $h=n-m+1, \ldots, n$ ) und die Steuerzahllasten $T_{h}^{u}$ gleich Null sind, können die Null-Profit-Bedingungen der befreiten Unternehmen $h$ als

$$
q_{h} Q_{h}-\sum_{j=1}^{n} a_{j h}\left(1+\tau_{j}^{u}\right) q_{j} Q_{h}=w L_{h}+r K_{h}
$$


dargestellt werden, woraus sich nach Division durch $Q_{h}$

$$
q_{h}=\sum_{j} a_{j h}\left(1+\tau_{j}^{u}\right) q_{j}+w l_{h}+r k_{h} \quad h=n-m+1, \ldots, n
$$

ergibt. Wie bereits erwähnt, gehen nun Brutto-Ausgaben für Vorleistungen in die Bestimmungsgleichung der Produzentenpreise befreiter Unternehmen ein. Fassen wir die Gleichungen (13) für $i=1, \ldots, n-m$ und (14) zusammen und wenden die analoge Vorgehensweise wie zuvor an, so ist wegen

$$
\begin{aligned}
q & =\left(A^{\prime}-A^{\prime}{ }_{m} \hat{\tau}^{u}\right) q+W_{e} \\
& =\left[\left(I-A^{\prime}\right)-A^{\prime}{ }_{m} \hat{\tau}^{u}\right]^{-1} W_{e}
\end{aligned}
$$

unschwer zu erkennen, daß nicht nur die Einheitsfaktorkosten, sondern auch die Umsatzsteuersätze (ungleich Null) die Höhe der Produzentenpreise bestimmen. Die Matrix $A^{\prime}{ }_{m}$ besteht hierbei in den Zeilen $n-m+1, \ldots, n$ aus den für befreite Unternehmen relevanten InputKoeffizienten, die restlichen Elemente sind gleich Null.

In der bisherigen Darstellung wurde aus Gründen der Übersichtlichkeit bewußt darauf verzichtet, die Produzentenpreise und die Faktorkosten pro Einheit Output, die sich bei Vorsteuerabzugsberechtigung aller Unternehmen ergeben, gesondert zu kennzeichnen, um sie von den entsprechenden Größen, die sich unter Berücksichtigung von Steuerbefreiungen einstellen, unterscheiden zu können. Dieses Vorgehen sollte jedoch nicht darüber hinwegtäuschen, daß sowohl Produzentenpreise als auch Einheitsfaktorkosten unter den beiden Besteuerungsalternativen verschieden hohe Werte annehmen. Bisher kam es auch nur darauf an, die unmittelbaren Bestimmungsfaktoren für die Produzentenpreise generell aufzuzeigen. Die Höhe der Variablen wird erst im folgenden Teil relevant, wenn wir Gleichung (15) den Bestimmungsgleichungen für Produzentenpreise gegenüberstellen, die sich bei Erhebung einer äquivalenten Konsumsteuer ergeben. Da der Steuerzugriff in diesem Fall erst dann erfolgt, wenn die Güter in den privaten Verbrauch gelangen (und nicht wenn sie als Zwischenprodukte verwendet werden), 
sind die Konsumsteuersätze offensichtlich kein Bestimmungsfaktor der Produzentenpreise. Aus den Null-Profit-Bedingungen

$$
p_{i} Q_{i}=T_{i}^{c}+\sum_{j=1}^{n} a_{j i} \tilde{q}_{j} Q_{i}+w L_{i}+r K_{i}
$$

mit $T_{i}^{c}$ als den von den Unternehmen zu entrichtenden Konsumsteuerbeträgen, erhalten wir über

$$
\begin{gathered}
\left(1+\tau_{i}^{c}\right) \tilde{q}_{i}=\tau_{i}^{c} \tilde{q}_{i}+\sum_{j=1}^{n} a_{j i} \tilde{q}_{j}+w l_{i}+r k_{i} \\
\tilde{q}_{i}=\sum_{j=1}^{n} a_{j i} \tilde{q}_{j}+w l_{i}+r k_{i}
\end{gathered}
$$

und in Matrixdarstellung schließlich

$$
\tilde{q}=\left(I-A^{\prime}\right)^{-1} W_{e} .
$$

Nach unserer Definition äquivalenter Steuersätze müssen die Faktorkosten $W_{e}$ des durch Gleichung (15) charakterisierten UmsatzsteuerGleichgewichts genauso hoch sein wie die Faktorkosten, die für die Unternehmen im Konsumsteuer-Gleichgewicht relevant sind. Wenn unter beiden Steuersystemen die gleichen Konsum- und Vorleistungsmengen nachgefragt werden, sind aufgrund der Produktionstechnologie die Faktoreinsatzmengen und damit auch die Faktorpreise identisch.

Nebenbei bemerkt, bestätigt der Vergleich von (15) und (16) die eingangs behauptete Aussage, daß die Produzentenpreise $q_{i}$ im Umsatzsteuer-Gleichgewicht von den Produzentenpreisen $\tilde{q}_{i}$ im Konsumsteuer-Gleichgewicht abweichen. Löst man beide Gleichungen nach $W_{e}$ auf, kann über

$$
\begin{gathered}
{\left[\left(I-A^{\prime}\right)-A^{\prime}{ }_{m} \hat{\tau}^{u}\right] q=\left(I-A^{\prime}\right) \tilde{q}} \\
\left(I-A^{\prime}\right)[q-\tilde{q}]=A^{\prime}{ }_{m} \hat{\tau}^{u} q
\end{gathered}
$$

die Differenz beider Preise ermittelt werden. Wir definieren diese Differenz $t^{u}$ durch

$$
t^{u}:=q-\tilde{q}=\left(I-A^{\prime}\right)^{-1} A^{\prime}{ }_{m} \hat{\tau}^{u} q .
$$


Sie stellt einen in den Produzentenpreisen $q$ implizit enthaltenen Umsatzsteuerbetrag pro Besteuerungseinheit dar. Da die Produzentenpreise nur positive Werte annehmen und auch die Input-Koeffizienten sowie die Umsatzsteuersätze nichtnegative Werte aufweisen, bestehen alle Vektoren bzw. Matrizen der Definitionsgleichung aus nichtnegativen Elementen ${ }^{60}$. Folglich sind auch die Steuerbeträge pro Besteuerungseinheit $t_{i}^{u}$ entweder streng positiv oder gleich Null.

Aus den $t_{i}^{u}$ und den beobachtbaren Produzentenpreisen $q_{i}$ können wiederum die fiktiven Produzentenpreise $\tilde{q}_{i}$ des Konsumsteuer-Gleichgewichts ermittelt werden. Mittels der fiktiven Produzentenpreise sind - im nächsten Schritt - die Steuerbeträge pro Besteuerungseinheit als implizite ad-valorem Steuersätze $\tilde{\tau}_{i}:=t_{i}^{u} / \tilde{q}_{i}$ darstellbar; sie genügen der Gleichung $q_{i}=\left(1+\tilde{\tau}_{i}\right) \tilde{q}_{i}$.

Eine identische Konsumnachfrage wird im Konsumsteuer- und im Umsatzsteuer-Gleichgewicht dann erreicht, wenn die Konsumentenpreise $p_{i}$ in beiden Fällen gleich hoch sind. Sie sind bei der Konsumsteuer durch $p_{i}=\left(1+\tau_{i}^{c}\right) \tilde{q}_{i}$, bei der Besteuerung der Umsätze durch $p_{i}=\left(1+\tau_{i}^{u}\right) q_{i}$ gegeben. Die effektiven Konsumsteuersätze $\tau_{i}^{c}$ können folglich über

$$
\begin{aligned}
\left(1+\tau_{i}^{c}\right) \tilde{q}_{i} & =\left(1+\tau_{i}^{u}\right) q_{i} \\
& =\left(1+\tau_{i}^{u}\right)\left(1+\tilde{\tau}_{i}\right) \tilde{q} \\
\tau_{i}^{c} & =\tau_{i}^{u}+\tau_{i}^{u} \tilde{\tau}_{i}+\tilde{\tau}_{i} \quad i=1, \ldots, n
\end{aligned}
$$

bestimmt werden. Die einzelnen Komponenten, aus denen sich der effektive Konsumsteuersatz zusammensetzt, lassen sich auch recht anschaulich interpretieren, wenn wir uns die Besteuerung des Konsums in mehreren Schritten vorstellen und dabei die zugrundeliegende Umsatzsteuer im Auge behalten. Zunächst wird auf den Produzentenpreis $\tilde{q}_{i}$ der nominelle Umsatzsteuersatz angewandt; $\tilde{\tau}_{i}$ erfaßt die Produzentenpreiserhöhung, die durch den Ausschluß des Vorsteuerabzugsrechts

${ }^{60}$ Betrachtet man $\left(I-A^{\prime}\right)^{-1}$ als unendliche geometrische Reihe $A+A^{2}+A^{3}+\ldots$, ist diese Aussage trivial. 
entstehen, und $\tau_{i}^{u} \tilde{\tau}_{i}$ entspricht der Anwendung des nominellen Umsatzsteuersatzes auf die steuerbedingte Preiserhöhung.

Aus Gleichung (18) geht in Verbindung mit Definition (17) weiterhin hervor, daß die von umsatzsteuerbefreiten (und nicht vorsteuerabzugsberechtigten) Unternehmen hergestellten Konsumgüter mit einem effektiven Steuersatz $\tau_{h}^{c}$ ungleich Null zu belegen wären, obwohl der nominelle Umsatzsteuersatz $\tau_{h}^{u}$ gleich Null ist. Wird dagegen allen Unternehmen der Abzug der Vorsteuer gestattet, sind alle Elemente der Matrix $A^{\prime}{ }_{m}$ gleich Null und damit ebenfalls die $t_{i}^{u}$ bzw. die impliziten Steuersätze $\tilde{\tau}_{i}$. Wir erhalten das bekannte Resultat, daß in diesem Fall Umsatz- und Konsumsteuersätze aufeinanderfallen.

\subsection{Ein numerisches Beispiel}

Zur Illustration der Berechnung effektiver Konsumsteuersätze wollen wir ein fiktives numerisches Beispiel mit nur 3 Unternehmen heranziehen. In diesem kleinen Rahmen bleiben die Gleichungen relativ überschaubar und können bequem nachvollzogen werden. Wir nehmen an, daß die Umsätze der Unternehmen 1 und 2 einer Steuer von $10 \%$ unterliegen, Unternehmen 3 sei dagegen steuerbefreit und nicht zum Abzug der Vorsteuer berechtigt. Aus der nachfolgenden InputOutput-Tabelle sind die einzelwirtschaftlichen Güter- bzw. Wertströme zu entnehmen.

Tabelle 2: Input-Output-Tabelle für ein numerisches Beispiel

\begin{tabular}{|c|ccc|c|c|}
\hline$V_{i j}$ & 1 & 2 & 3 & $q_{i} C_{i}$ & $q_{i} Q_{i}$ \\
\hline 1 & 15 & 20 & 25 & 40 & 100 \\
2 & 20 & 15 & 30 & 35 & 100 \\
3 & 30 & 35 & 10 & 25 & 100 \\
\hline$N U_{i}$ & 0 & 0 & 5.5 & 7.5 & \multicolumn{1}{|}{} \\
\cline { 1 - 3 }$w L_{i}$ & 17 & 14 & 17.5 & & \\
$r K_{i}$ & 18 & 16 & 12 & & \\
\cline { 1 - 4 }$q_{i} Q_{i}$ & 100 & 100 & 100 & &
\end{tabular}


Der Ausweis der Umsatzsteuer erfolgt analog zur Vorgehensweise des Statistischen Bundesamtes. Alle Wertströme werden netto - d. h. ohne Umsatzsteuern - verbucht, letztere sind in einer Zeile unterhalb der Zentralmatrix und der Matrix der Endnachfrage denjenigen Unternehmen oder Endnachfragekategorien zugeordnet, die sie in dem betrachteten Zeitraum zu tragen hatten ${ }^{61}$. Danach können der Tabelle keine an das Finanzamt zu entrichtenden Steuerzahllasten $T_{i}^{u}$ entnommen werden, sondern nur diejenigen Steuerbeträge $N U_{i}$, die der jeweilige Käufer der Güter nicht geltend machen kann. In unserem Beispiel besteht $N U$ aus den nichtabsetzbaren Vorsteuern des Unternehmens 3 $\left(N U_{3}^{u}=\tau_{1}^{u} q_{1} A_{13}+\tau_{2}^{u} q_{2} A_{23} ; N U_{1}^{u}=N U_{2}^{u}=0\right)$ und der auf die Konsumausgaben anfallenden Steuer $\left(N U^{c}=\tau_{1}^{u} q_{1} C_{1}+\tau_{2}^{u} q_{2} C_{2}\right)$. Die Summe der nichtabzugsfähigen Umsatzsteuer ergibt das gesamtwirtschaftliche Steueraufkommen

$$
T^{u}=\tau_{1}^{u} q_{1} C_{1}+\tau_{2}^{u} q_{2} C_{2}+\tau_{1}^{u} q_{1} A_{13}+\tau_{2}^{u} q_{2} A_{23} .
$$

Das gleiche Resultat ließe sich - nebenbei bemerkt - auch durch Aufsummierung der einzelwirtschaftlichen Steuerzahllasten $T_{i}^{u}$ erzielen (siehe Abschnitt (1) Gleichung (7)).

Zur Trennung der in der Tabelle angegebenen Wertgrößen in Preisund Mengenkomponente unterstellen wir ferner, daß alle Produzentenund Faktorpreise gleich Eins sind. Die Input-Koeffizientenmatrix $A$ ist damit durch

$$
A=\left(\begin{array}{lll}
0.15 & 0.20 & 0.25 \\
0.20 & 0.15 & 0.30 \\
0.30 & 0.35 & 0.10
\end{array}\right)
$$

gegeben.

Setzt man anstelle der Input-Koeffizienten in allgemeiner Darstellung die tatsächlichen Werte ein, erhalten die Null-Profit-Bedingungen (13) bzw. (14) folgende Gestalt:

$$
\begin{aligned}
& q_{1}-\quad 0.15 q_{1}-\quad 0.20 q_{2}-0.30 q_{3}=w l_{1}+r k_{1} \\
& q_{2}-\quad 0.20 q_{1}-\quad 0.15 q_{2}-0.35 q_{3}=w l_{2}+r k_{2} \\
& q_{3}-0.25\left(1+\tau_{1}^{u}\right) q_{1}-0.30\left(1+\tau_{2}^{u}\right) q_{2}-0.10 q_{3}=w l_{3}+r k_{3}
\end{aligned}
$$

${ }^{61}$ Siehe bspw. ST BA [1988], S. 20 f. 
Nach einer Zusammenfassung des obigen Gleichungssystems in Matrixform und dessen Auflösung nach Produzentenpreisen, erhalten wir mit

$$
\begin{aligned}
\left(\begin{array}{l}
q_{1} \\
q_{2} \\
q_{3}
\end{array}\right)= & {\left[\left(\begin{array}{ccc}
1-0.15 & -0.20 & -0.30 \\
-0.20 & 1-0.15 & -0.35 \\
-0.25 & -0.30 & 1-0.10
\end{array}\right)-\right.} \\
& \left.-\left(\begin{array}{ccc}
0 & 0 & 0 \\
0 & 0 & 0 \\
0.25 \tau_{1}^{u} & 0.30 \tau_{2}^{u} & 0
\end{array}\right)\right]^{-1}\left(\begin{array}{l}
w l_{1}+r k_{1} \\
w l_{2}+r k_{2} \\
w l_{3}+r k_{3}
\end{array}\right)
\end{aligned}
$$

die zu (15) analoge Gleichung. Die zweite Matrix innerhalb der eckigen Klammer entspricht hierbei dem ausmultiplizierten Term $A^{\prime}{ }_{m} \hat{\tau}$, wobei $\tau_{3}^{u}=0$ bereits berücksichtigt wurde. Aus Definition (17) lassen sich schließlich die Steuerbeträge pro Besteuerungseinheit als

$$
\left(\begin{array}{c}
t_{1}^{u} \\
t_{2}^{u} \\
t_{3}^{u}
\end{array}\right)=\left(\begin{array}{ccc}
1-0.15 & -0.20 & -0.30 \\
-0.20 & 1-0.15 & -0.35 \\
-0.25 & -0.30 & 1-0.10
\end{array}\right)^{-1}\left(\begin{array}{c}
0 \\
0 \\
0.25 \tau_{1}^{u}+0.30 \tau_{2}^{u}
\end{array}\right)
$$

bestimmen. Die nachfolgenden Schritte sind offensichtlich: Aus der Differenz zwischen den Produzentenpreisen $q_{i}$ und den Steuerbeträgen $t_{i}^{u}$ können die Produzentenpreise $\tilde{q}_{i}$ des Konsumsteuer-Gleichgewichts ermittelt werden, daraus wiederum die impliziten und die effektiven Steuersätze.

In Tabelle 3 sind die nominellen Umsatzsteuersätze den berechneten effektiven Konsumsteuersätzen gegenübergestellt. Als zusätzliche Information werden daneben die beobachtbaren Produzentenpreise, die impliziten Steuersätze und die Produzentenpreise $\tilde{q}_{i}$ des KonsumsteuerGleichgewichts aufgelistet.

Man erkennt sofort, daß die effektiven Steuersätze $\tau_{i}^{c}$ durchweg höher sind als die nominellen $\tau_{i}^{u}$. Am deutlichsten fällt der Unterschied bei Unternehmen 3 aus. Obwohl die Umsätze des Unternehmens steuerbefreit sind, müßten die Konsumausgaben im äquivalenten Konsumsteuer-Gleichgewicht mit einer Steuer von nahezu $10 \%$ belegt werden (wegen $\tau_{3}^{u}=0$ sind in diesem Fall auch impliziter und effektiver Steuersatz identisch). Damit kann der implizite Steuersatz $\tilde{\tau}_{3}$ 
Tabelle 3: Effektive Steuersätze für ein numerisches Beispiel

\begin{tabular}{|c|c|c|c|c|c|}
\hline \multirow{2}{*}{ Sektoren } & \multicolumn{3}{|c|}{ Steuersätze (in \%) } & \multicolumn{3}{|c|}{ Preise } \\
& $\tau_{i}^{u}$ & $\left.\tau_{i}^{c}{ }^{\bullet}\right)$ & $\left.\tilde{\tau}^{*}\right)$ & $q_{i}$ & $\tilde{q}_{i}{ }^{\bullet}$ \\
\hline 1 & 10 & 14.82 & 4.38 & 1.0 & 0.958 \\
2 & 10 & 15.33 & 4.84 & 1.0 & 0.954 \\
3 & 0 & 9.67 & 9.67 & 1.0 & 0.912 \\
\hline
\end{tabular}

*) gerundete Werte

als Indikator der durch den Ausschluß des Vorsteuerabzugsrechts ausgelösten Nachholwirkung der Umsatzsteuer aufgefaßt werden. Diese Nachholwirkung beschränkt sich aber nicht nur auf Unternehmen 3 - wie es möglicherweise Gleichung (19) vermuten ließe -, sie erreicht vielmehr alle Unternehmen. Trotz Vorsteuerabzugsberechtigung der Unternehmen 1 und 2, sind deren Produzentenpreise im UmsatzsteuerGleichgewicht mit impliziten Steuersätzen in einer Größenordnung von ungefähr $4.5 \%$ behaftet.

Ein weiterer Punkt geht aus dem Beispiel unmittelbar hervor. Die nominellen Umsatzsteuersätze sind so gestaltet, daß der Konsum von Gut 3 steuerlich begünstigt wird, während für Gut 1 und 2 ein einheitlicher Steuersatz zur Anwendung kommt. Die Betrachtung der effektiven Konsumsteuersätze zeigt aber, daß $C_{2}$ mit einem höheren effektiven Steuersatz behaftet ist wie $C_{1}$. Diese unbeabsichtigte Diskriminierung von Gut 2 gegenüber Gut 1 ist (einzig) auf die Steuerbefreiung des dritten Unternehmens zurückzuführen und nicht vom Gesetzgeber beeinflußbar. Damit kann aber die allokative Wirkung der Steuer (wie auch die Verteilungseffekte) nur bedingt vom Fiskus gesteuert werden, falls das Umsatzsteuersystem unechte Befreiungen enthält. Dieser negative Seiteneffekt wird gegen Befreiungen unter Ausschluß des Vorsteuerabzugsrechts ins Felde geführt. Es wäre jedoch voreilig aus dem eher steuerungspolitischen Argument eine nachteilige Wirkung auf die Effizienz abzuleiten. Dieser Punkt wird in Kapitel IV behandelt. 
Zur Herleitung der allgemeinen Berechnungsformel für äquivalente Konsumsteuersätze sind nur die Null-Profit-Bedingungen der Unternehmen und die Annahme von konstanten Input-Koeffizienten notwendig. Die Konstruktion der effektiven Steuersätze stellt sicher, da $B$ in beiden Gleichgewichten identische Steueraufkommen - und damit identische Transferzahlungen -, identische Konsumentenpreise und identische Faktorkosten bzw. -einkommen erzielt werden. Damit haben sich die Rahmenbedingungen, denen sich der nutzenmaximierende Haushalt und die gewinnmaximierenden Unternehmen gegenübersehen, nicht verändert. Es besteht demnach keine Notwendigkeit spezielle Anforderungen an die Nutzenfunktion des Haushalts und die Wertschöpfungsfunktionen der Unternehmen zu stellen. An dieser Stelle sei ebenfalls darauf hingewiesen, daß eine auf der Basis der in Tabelle 2 gegebenen Daten durchgeführte Simulationsrechnung die Äquivalenz der Steuersätze auch numerisch bestätigte. Für diese Verifikation im Rahmen eines Empirischen Allgemeinen Gleichgewichtsmodells war es allerdings notwendig, konkrete funktionelle Formen zu spezifizieren. Die unterstellten Funktionstypen, wie auch die gewählten exogenen Parameterwerte können Anhang 3 entnommen werden.

Unser Beispiel verdeutlicht die Unterschiede zwischen effektiver und nomineller Steuer recht drastisch, was sicherlich auf die gewählten $\mathrm{Ba}$ sisdaten zurückzuführen ist. Beispielsweise sind die von Unternehmen 3 eingesetzten besteuerten Vorleistungen relativ hoch und die Verwendung des dritten Gutes in der Produktion der Güter 1 und 2 sehr intensiv. Legt man eine etwas realistischere Tabelle zugrunde, dürften die Diskrepanzen in den Steuersätzen nicht so dramatisch ausfallen. Das Beispiel diente - neben einer Veranschaulichung der im vorangehenden Abschnitt verwendeten relativ kompakten Darstellungsweise nicht zuletzt auch dem Zweck, die sich ergebenden Unterschiede plastisch werden zu lassen. Bevor wir uns jedoch realistischeren Zahlen zuwenden, wollen wir sukzessive Außenhandelsbeziehungen und Investitionstätigkeiten in das Modell aufnehmen. 


\subsection{Außenhandel}

Die Erweiterung des Grundmodells um außenwirtschaftliche Beziehungen beschränkt sich auf die produzierten Güter; die Primärfaktoren Arbeit und Kapital werden dagegen weiterhin als international immobil unterstellt ${ }^{62}$. Wie zuvor ist es für die Berechnung der effektiven Steuersätze nicht notwendig, spezifische Verhaltensgleichungen hinsichtlich des Importangebots und der Exportnachfrage des Auslandes zu formulieren. Wir nehmen lediglich an, daß beide Funktionen von den inländischen Preisen abhängen und darüber hinaus Importgüter vollkommene Substitute zu den entsprechenden inländischen Produkten darstellen. Die Konsequenzen dieser Annahme werden im weiteren Verlauf an den betreffenden Stellen hervorgehoben.

Unter Berücksichtigung von Außenhandel sind die Gleichgewichtsbedingungen für den Gütermarkt durch

$$
\begin{aligned}
Q_{i}+M_{i} & =\sum_{j=1}^{n} A_{i j}^{I}+\sum_{j=1}^{n} A_{i j}^{M}+C_{i}^{I}+C_{i}^{M}+X_{i} \quad i=1, \ldots, n \\
\operatorname{mit} & \sum_{j=1}^{n} A_{i j}^{I}+\sum_{j=1}^{n} A_{i j}^{M}=\sum_{j=1}^{n} a_{i j} Q_{j} \quad \text { und } C_{i}^{i}+C_{i}^{M}=C_{i}
\end{aligned}
$$

gegeben. Die linke Seite der Gleichung repräsentiert das aus inländischen Erzeugnissen $Q_{i}$ und importierten Gütern $M_{i}$ bestehende Güteraufkommen, die rechte Seite zeigt deren Verwendung. Index $I$ kennzeichnet hierbei inländische, Index $M$ importierte Güter.

Die Besteuerung des grenzüberschreitenden Güterverkehrs erfolgt nach dem in Kapitel I erläuterten Bestimmungslandprinzip. Alle Importe werden mit einem Einfuhrumsatzsteuersatz belegt, der dem auf vergleichbare heimische Netto-Umsätze angewandten Steuersatz entspricht, die Exporte sind generell von der Umsatzsteuer befreit. Durch die Einfuhrumsatzsteuer entsteht ein Aufkommen in Höhe von

$$
T_{M}^{u}=\sum_{i=1}^{n} \tau_{i}^{u} q_{i} M_{i}
$$

${ }^{62} \mathrm{Da}$ diese Annahme keinen Einfluß auf die Ermittlung effektiver Steuersätze ausübt - man könnte ebensogut von international mobilen Primärfaktoren ausgehen -, wird die einfachere Variante gewählt. 
Aus dieser Darstellung geht beispielsweise hervor, daß importierte Produkte zu denselben Preisen gehandelt werden wie inländische. Der Grund hierfür ist einfach gefunden. Wenn Importgüter absolut gleichwertig zu inländischen Gütern sind, muß der (gleichgewichtige) Bruttopreis für ein importiertes Produkt ebenso hoch sein wie der Bruttopreis des entsprechenden heimischen Erzeugnisses - bei unterschiedlichen Preisen wäre das jeweils teurere nicht konkurrenzfähig. Da auf beide ferner derselbe Steuersatz angewandt wird, sind auch die Nettopreise identisch.

Die Steuerzahllast eines steuerpflichtigen und zum Abzug der Vorsteuer berechtigten Unternehmens kann nun über

$$
T_{i}^{u}=\tau_{i}^{u} q_{i}\left(\left(1-a_{i i}\right) Q_{i}-X_{i}\right)-\sum_{j \neq i} a_{j i} \tau_{j}^{u} q_{j} Q_{i}
$$

ermittelt werden. Man bemerkt, daß nur die Exporte in Gleichung (21) explizit aufgeführt sind; sie vermindern die Basis-Umsatzsteuerschuld. Die Importe sind implizit in den insgesamt eingesetzten Vorleistungen (exklusive Eigenverbrauch) enthalten.

$\mathrm{Da}$ das Vorsteuerabzugsrecht im allgemeinen weder damit verbunden wird, ob ein inländisches Unternehmen seine Erzeugnisse exportiert oder stattdessen an inländische Abnehmer verkauft ${ }^{63}$, noch davon abhängt, in welchem Ausmaß das Unternehmen importierte Zwischenprodukte verwendet, ist es zur Ermittlung der abzugsfähigen Vorsteuer nicht notwendig zwischen inländischen und importierten Vorleistungen $\mathrm{zu}$ unterscheiden. Ausschlaggebend sind allein die insgesamt eingesetzten Vorleistungsmengen und diese werden durch die InputKoeffizienten $a_{j i}(\forall j \neq i)$ festgelegt.

Unterstellt man generelles Vorsteuer-Abzugsrecht für alle Unternehmen, erhält man durch Aufsummierung der einzelwirtschaftlichen Steu-

${ }^{63}$ Wie in Kapitel I, Abschnitt (3) erläutert wurde, ist es steuerbefreiten (und nicht abzugsberechtigten) Unternehmen unter bestimmten Umständen gestattet, die Vorsteuern, die unmittelbar auf die Produktion von Exporten in DrittLänder anfallen, geltend zu machen. Derartige Sonderregelungen werden jedoch nicht berücksichtigt. 
erzahllasten und der Einfuhrumsatzsteuer

$$
\begin{aligned}
T^{u} & =T_{M}^{u}+\sum_{i=1}^{n} T_{i}^{u} \\
& =\sum_{i=1}^{n} \tau_{i}^{u} q_{i} M_{i}+\sum_{i=1}^{n} \tau_{i}^{u} q_{i}\left(Q_{i}-X_{i}\right)-\sum_{i=1}^{n} \sum_{j=1}^{n} a_{j i} \tau_{j}^{u} q_{j} Q_{i} \\
& =\sum_{i=1}^{n} \tau_{i}^{u} q_{i}\left[\left(Q_{i}-X_{i}\right)+M_{i}-\sum_{j=1}^{n} a_{i j} Q_{j}\right]
\end{aligned}
$$

Unter Berücksichtigung der Marktgleichgewichtsbedingungen (20) folgt daraus unmittelbar

$$
T^{u}=\sum_{i=1}^{n} \tau_{i}^{u} q_{i} C_{i} .
$$

Bei Anwendung des Bestimmungslandprinzips wird demnach wiederum nur der Konsum besteuert.

Betrachten wir die Null-Profit-Bedingungen, die nun in allgemeiner Form durch

$$
\begin{gathered}
\left(1+\tau_{i}^{u}\right) q_{i}\left(\left(1-a_{i i}\right) Q_{i}-X_{i}\right)+q_{i} X_{i}=T_{i}^{u}+\sum_{j \neq i} a_{j i}\left(1+\tau_{j}^{u}\right) q_{j} Q_{i}+ \\
+w L_{i}+r K_{i},
\end{gathered}
$$

gegeben sind, ist nach Einsetzen von Gleichung (21) in (22) unschwer zu erkennen, daß sich für abzugsberechtigte Firmen dieselben Gleichungen zur Bestimmung der Produzentenpreise einstellen wie in Abschnitt 3 (Gleichungen (13)). Setzt man in Gleichung (22) $\tau_{i}^{u}$ und $T_{i}^{u}$ gleich Null, wird ebenfalls deutlich, daß auch befreite und nicht vorsteuerabzugsberechtigte Unternehmen ihre Produzentenpreise weiterhin nach Gleichung (14) kalkulieren.

Damit erhalten wir dieselben Voraussetzungen zur Berechnung der effektiven Steuersätze und - als logische Konsequenz davon - auch dieselben Berechnungsformeln wie für das Modell der geschlossenen 
Volkswirtschaft. Es wäre müßig die einzelnen Schritte zu wiederholen. $\mathrm{Zu}$ beachten ist jedoch, daß die Erhebung effektiver Konsumsteuersätze allein nicht mehr ausreicht, um eine zum Umsatzsteuer-Gleichgewicht identische Allokation zu erzielen.

Bekanntlich stellen sich im Konsumsteuer-Gleichgewicht die Produzentenpreise $\tilde{q}_{i}$ ein, während die Erhebung einer Umsatzsteuer die Produzentenpreise $q_{i}=\left(1+\tilde{\tau}_{i}\right) \tilde{q}_{i}$ hervorbringt. Vergegenwärtigen wir uns, daß die Exportnachfrage des Auslands von den Produzentenpreisen abhängt, wird klar, daß im Konsumsteuer-Gleichgewicht die Exportmengen nur dann genauso hoch sind wie im UmsatzsteuerGleichgewicht, wenn neben die Besteuerung des Konsums eine Exportsteuer tritt. Die Exportsteuersätze $\tau_{i}^{X}$ entsprechen hierbei den impliziten Steuersätzen $\tilde{\tau}_{i}$, so daß der Bruttopreis für Exportgüter $q_{i}=\left(1+\tau_{i}^{X}\right) \tilde{q}_{i}$ beträgt.

Eine analoge Argumentationslinie trifft für das Importangebot des Auslands zu - allerdings mit umgekehrten Vorzeichen. Importmengen in Höhe von $M_{i}$ sind im Konsumsteuer-Gleichgewicht nur zu den Produzentenpreisen $q_{i}$ erreichbar. Aufgrund der vollkommenen Substituierbarkeit von heimischen und importierten Produkten würde jedoch kein Wirtschaftssubjekt Importgüter zu diesem Preis kaufen, da gleichwertige inländische Produkte zum niedrigeren Nettopreis $\tilde{q}_{i}$ angeboten werden. Damit Importgüter konkurrenzfähig bleiben, gleicht der Staat diesen Preisvorteil inländischer Güter durch Subventionierung der Importe aus. Die Subventionssätze orientieren sich logischerweise ebenfalls an den impliziten Steuersätzen. Die ausländischen Exporteure erzielen somit die Erträge $\sum q_{i} M_{i}$, für inländische Abnehmer sind durch die Subventionierung jedoch nur die Nettopreise $\left(1-\tau_{i}^{M}\right) q_{i}=\tilde{q}_{i}$ mit $\tau_{i}^{M}=\tilde{\tau}_{i} /\left(1+\tilde{\tau}_{i}\right)$ maßgebend. Ein (inländisches) Unternehmen $i$ zahlt deshalb auf importierte Vorprodukte effektiv den Betrag $\sum \tilde{q}_{j} A_{j i}^{M}$, dem repräsentativen Haushalt entstehen durch den Kauf von Importgütern Ausgaben in Höhe von $\left(1+\tau_{i}^{c}\right) \tilde{q}_{i} C_{i}^{M}$.

Das zur ursprünglichen Umsatzsteuer äquivalente Steuersystem besteht demnach aus einer Besteuerung des Konsums mit den Steuersätzen $\tau_{i}^{c}$, einer Exportsteuer mit den Sätzen $\tau_{i}^{X}$ und einer Subventionierung der Importe mit den auf die Importgüter-Bruttopreise bezogenen Subventionssätzen $\tau_{i}^{M}$. Entgegen der vorherrschenden Mei- 
nung, daß sich die Umsatzsteuer bei Anwendung des Bestimmungslandprinzips auch hinsichtlich des Außenhandels neutral verhält - teilweise wird sogar die Ansicht vertreten, daß sie aus der Sicht des Inlands Exporte begünstigt und Importe benachteiligt -, zeigt die Betrachtung des äquivalenten Steuersystems eine Diskriminierung der Exporte und eine Begünstigung der Importe auf ${ }^{64}$.

Wie diese Wirkung der Umsatzsteuer zustande kommt, kann auch anhand der ökonomischen Reaktionsabläufe verdeutlicht werden, die entstehen, wenn man von einem Umsatzsteuersystem, das ausschließlich Nullsteuersätze vorsieht, auf unechte Befreiungen übergeht. Durch den Wegfall des Vorsteuer-Abzugsrechts verteuern sich zunächst die Erzeugnisse der befreiten Unternehmen $h(=n-m+1, \ldots, n)$. Dabei spielt es keine Rolle, ob deren Vorsteuer auf die Bezüge inländischer oder importierter Zwischenprodukte angefallen ist. Nachgelagerte Unternehmen und der repräsentative Haushalt werden daraufhin diese Produkte durch Importgüter substituieren. Die Nachfrage für Importgüter $h$ steigt, während die Nachfrage für gleichwertige inländische Erzeugnisse zurückgeht. Unterstellt man einen normalen Verlauf der Importangebotsfunktion $\left(\partial q_{i} / \partial M_{i}>0\right)$ steigt der Preis für Importgüter, wohingegen der Preis der heimischen Produkte sinkt. Dieser Prozeß setzt sich solange fort, bis ein einheitlicher Preis pro Gut herrscht. Da nachgelagerte Unternehmen aufgrund der Verteuerung der von ihnen benötigten Zwischenprodukte ihre Produzentenpreise ebenfalls revidieren müssen, ist der gesamte Produktionssektor einschließlich der Importe von der tendenziellen Preissteigerung betroffen. Sie führt dazu, daß mehr Importe zu einem höheren Preis nachgefragt werden als bei der Erhebung von Nullsteuersätzen und auf der anderen Seite Exporte zu diesem höheren Preis in geringeren Mengen nachgefragt werden. Die naheliegende Vermutung, daß Umsatzsteuerbefreiungen unter Ausschluß des Vorsteuerabzugsrechts aus den genannten Gründen die in-

${ }^{64}$ Um Verwechslungen vorzubeugen, sei an dieser Stelle nochmals betont, daß die Handelsneutralität des Bestimmungslandprinzips ausschließlich auf die steuerliche Gleichbehandlung von inländischen und importierten Gütern bzw. die im Rahmen der GATT-Bestimmungen erlaubte Freistellung der Exporte von indirekten Steuern abzielt. Damit ist nicht gemeint, daß eine bestimmte Struktur der Umsatzsteuersätze keinerlei Wirkungen auf den Außenhandel zeitigen würde. 
ternationale Wettbewerbsposition des besteuernden Landes verschlechtern, muß jedoch in einem Gleichgewichtsmodell geprüft werden. Denn die beschriebenen Reaktionsabläufe führen zunächst zu einer ungleichgewichtigen Leistungsbilanz, wodurch zusätzliche Anpassungsprozesse ausgelöst werden.

\subsection{Investitionen}

Im vorangehenden Abschnitt wurde gezeigt, wie effektive Steuersätze unter Berücksichtigung von Außenhandel zu berechnen sind. Um die bislang noch unberücksichtigte Güterverwendungsart - die Investitionen - in das Modell zu integrieren, werden wir die Annahme eines gesamtwirtschaftlich fixen Kapitalstocks durch einige grundlegende wachstumstheoretische Zusammenhänge ersetzen. Auf eine kurze Beschreibung dieser Modellerweiterung folgt erneut die Berechnung der effektiven Steuersätze. Dabei erweist es sich als besonders günstig, daß die zentrale Bestimmungsgleichung für effektive Steuersätze durch die Modellerweiterung um Außenhandelsbeziehungen keiner Veränderung bedurfte. Wir können deshalb auf die Modellierung von Außenhandel in diesem Abschnitt verzichten, ohne dadurch wesentliche Einschränkungen hinnehmen zu müssen.

Die Nachfrage nach Investitionen wird durch den produktionsbedingten Verschleiß von Kapital und die gewünschte Kapitalstock-VergröBerung bestimmt. Aus der neoklassischen Wachstumstheorie ist bekannt, daß die Netto-Investitionen im gleichgewichtigen Wachstumspfad ebenso hoch sind wie die exogene Wachstumsrate $g$ des Arbeitskräftepotentials ${ }^{65}$. Die (gleichgewichtigen) Netto-Investitionen $\dot{K}_{i}$ eines Unternehmens $i$ sind somit durch $\dot{K}_{i}=g K_{i}$ bestimmt. Unterstellen wir ferner, daß die Reinvestitionen einen konstanten, aber sektorspezifischen Anteil $\delta_{i}$ des eingesetzten Kapitalstocks $K_{i}$ ausmachen, ist das von Unternehmen $i$ insgesamt nachgefragte Investitionsvolumen durch $\left(g+\delta_{i}\right) K_{i}$ gekennzeichnet. Finanziert werden die Investitionen durch Ausgabe von Wertpapieren.

Keines der Unternehmen stellt jedoch direkt Kapitalgüter her, sondern alle produzierten Güterarten werden neben ihrer Verwendung als

${ }^{65}$ Siehe bspw. KROMPHARDT [1977], S. $81 \mathrm{ff}$. 
Zwischenprodukt oder Konsumgut nun auch für investive Zwecke eingesetzt. Erst eine für alle Unternehmen identische Transformationsfunktion verwandelt diese angebotenen Investitionsgüter in den homogenen Produktionsfaktor Kapital. Letzterer stellt damit eine Art composite commodity dar, das sich aus fixen Anteilen $\beta_{i}\left(\right.$ mit $\left.\sum_{i=1}^{n} \beta_{i}=1\right) \operatorname{der}$ verschiedenen Investitionsgüterarten zusammensetzt.

Mit Hilfe dieser Transformationsfunktion kann das Investitionsvolumen in eine Nachfrage für die einzelnen Investitionsgüter aufgespaltet werden. Die von Unternehmen $j$ nachgefragte Menge des Investitionsgutes $i$ ist somit durch

$$
I_{i j}=\beta_{i}\left(g+\delta_{j}\right) K_{j} \quad i, j=1, \ldots, n
$$

bestimmt. Die gesamtwirtschaftliche Nachfrage $I_{i}$ nach Investitionsgut $i$ erhält man, indem $I_{i j}$ über alle Unternehmen $j$ aufsummiert wird. Damit lassen sich die Markträumungsbedingungen durch

$$
Q_{i}=\sum_{j=1}^{n} a_{i j} Q_{j}+C_{i}+I_{i} \quad i=1, \ldots, n
$$

beschreiben.

Bei einer Umsatzsteuer vom Konsumtyp sind neben den Vorsteuern auf Zwischenprodukte auch die Steuern, die auf den Kauf von Investitionsgüter anfallen, abzugsfähig. Da mit dem Einsatz von Investitionsgütern, die aus der eigenen Produktion stammen, keine Markttransaktionen verbunden sind, berechnet ein vorsteuerabzugsberechtigtes Unternehmen $i$ seine Steuerzahllast über

$$
\begin{aligned}
T_{i}^{u} & =\tau_{i} q_{i}\left(\left(1-a_{i i}\right) Q_{i}-I_{i i}\right)-\sum_{j \neq i} \tau_{j} q_{j} a_{j i} Q_{i}-\sum_{j \neq i} \tau_{j} q_{j} I_{j i} \\
& =\tau_{i} q_{i} Q_{i}-\sum_{j=1}^{n} \tau_{j} q_{j} a_{j i} Q_{i}-\sum_{j=1}^{n} \tau_{j} q_{j} I_{j i} .
\end{aligned}
$$

Unter Verwendung der Markträumungsbedingungen (23) kann wiederum gezeigt werden, daß die Umsatzsteuer bei Vorsteuerabzugsbe- 
rechtigung aller Unternehmen nur die Konsumausgaben trifft. Aufsummierung der Steuerzahllasten ergibt

$$
\begin{aligned}
T^{u}=\sum_{i=1}^{n} T_{i}^{u} & =\sum_{i=1}^{n} \tau_{i}^{u} q_{i} Q_{i}-\sum_{i=1}^{n} \sum_{j=1}^{n} \tau_{j}^{u} q_{j} a_{i j} Q_{i}-\sum_{i=1}^{n} \sum_{j=1}^{n} \tau_{j}^{u} q_{j} I_{i j} \\
& =\sum_{i=1}^{n} \tau_{i}^{u} q_{i}\left(Q_{i}-\sum_{j=1}^{n} a_{i j} Q_{j}-I_{i}\right),
\end{aligned}
$$

was letztendlich $\mathrm{zu}$

$$
T^{u}=\sum_{i=1}^{n} \tau_{i}^{u} q_{i} C_{i}
$$

führt.

Sind dagegen $m$ der $n$ Unternehmen steuerbefreit und vom Abzug der Vorsteuer ausgeschlossen, läßt das analog ermittelte gesamtwirtschaftliche Steueraufkommen

$$
T^{u}=\sum_{i=1}^{n} \tau_{i}^{u} q_{i} C_{i}+\sum_{h=n-m+1}^{n} \sum_{i=1}^{n} a_{i h} \tau_{i}^{u} q_{i} Q_{h}+\sum_{h=n-m+1}^{n} \sum_{i=1}^{n} \tau_{i}^{u} q_{i} I_{h i}
$$

in diesem Fall erkennen, daß auch die Investitionen, die von befreiten Unternehmen nachgefragt werden, mit Umsatzsteuern behaftet sind.

Die für befreite Unternehmen maßgeblichen Null-Profit-Bedingungen lassen sich deshalb durch

$$
\dot{Z}+q_{h} Q_{h}=\sum_{j=1}^{n} a_{j h} p_{j} Q_{h}+\sum_{j=1}^{n} \beta_{j} p_{j}\left(g+\delta_{h}\right) K_{h}+r Z_{h}+w L_{h}
$$

mit $h=n-m+1, \ldots, n$ darstellen. Zusammen mit der Neuausgabe von Wertpapieren $\dot{Z}\left(=\sum_{j=1}^{n} \beta_{j} p_{j}\left(g+\delta_{h}\right) K_{h}\right)$ müssen die Unternehmenserträge $q_{h} Q_{h}$ die laufenden Produktionskosten, die Ausgaben für Investitionsgüter sowie die Dividendenzahlungen $r Z_{h}$ auf den Bestand an 
Wertpapieren $Z_{h}$ decken. Löst man die Null-Profit-Bedingungen nach den Produzentenpreisen $q_{h}$ auf, erhält man

$$
\begin{aligned}
q_{h} & =\sum_{j=1}^{n} a_{j h} p_{j}+\sum_{j=1}^{n} p_{j} \beta_{j}\left(g+\delta_{h}\right) k_{h}+r z_{h}-\dot{z}_{h}+w l_{h} \\
& =\sum_{j=1}^{n} a_{j h} p_{j}+p^{I}\left(g+\delta_{h}\right) k_{h}+r z_{h}-\dot{z}_{h}+w l_{h}
\end{aligned}
$$

wobei $z_{h}=Z_{h} / Q_{h}$ und $\dot{z}_{h}=\dot{Z}_{h} / Q_{h}$ den Wertpapierbestand und die Wertpapieremission pro Einheit Output angeben. Wie für die Vorleistungen sind auch für die einzelnen Investitionsgüter Bruttopreise zu zahlen. Wir können deshalb $p^{I}:=\sum_{j} p_{j} \beta_{j}$ als BruttoAnschaffungspreis für Kapitalgüter betrachten ${ }^{66}$.

Für abzugsberechtigte Unternehmen haben die Null-Profit-Bedingungen dagegen die Form

$$
\dot{Z}_{i}+p_{i} Q_{i}=T_{i}^{u}+\sum_{j=1}^{n} a_{j i} p_{j} Q_{i}+\sum_{j=1}^{n} \beta_{j} p_{j}\left(g+\delta_{i}\right) K_{i}+r Z_{i}+w L_{i}
$$

mit $i=1, \ldots, n-m$, woraus nach Einsetzen von Gleichung (24) und Division durch den Unternehmensoutput die Bestimmungsgleichungen der Produzentenpreise

$$
\begin{aligned}
q_{i} & =\sum_{j=1}^{n} a_{j i} q_{j}+\sum_{j=1}^{n} q_{j} \beta_{j}\left(g+\delta_{i}\right) k_{i}+r z_{i}-\dot{z}_{i}+w l_{i} \\
& =\sum_{j=1}^{n} a_{j i} q_{j}+q^{I}\left(g+\delta_{i}\right) k_{i}+r z_{i}-\dot{z}_{i}+w l_{i}
\end{aligned}
$$

${ }^{66} \mathrm{Da}$ der Bruttopreis nur für befreite und nicht vorsteuerabzugsberechtigte Unternehmen $h$ berechnet wird, sind wegen $\tau_{h}=0$ automatisch auch die selbstproduzierten Investitionsgüter $I_{h h}$ mit dem richtigen Güterpreis bewertet. 
folgen. Ganz analog wird hierbei $q^{I}:=\sum_{j} \beta_{j} q_{j}$ als Netto-Anschaffungspreis für Kapital definiert.

Betrachten wir die Gleichungen (25) und (26), liegt das Vorgehen nahe die beiden Preisindizes $p^{I}$ und $q^{I}$ über einen nominellen Investitionssteuersatz $\tau_{h}^{I}$ mit

$$
\tau_{h}^{I}:=\frac{t_{h}^{I}}{q^{I}}=\frac{\sum_{i} \beta_{i} \tau_{i}^{u} q_{i}}{\sum_{i} \beta_{i} q_{i}}
$$

zu verbinden. Über diese Definition ließe sich bspw. der zweite Term auf der rechten Seite von Gleichung (25) durch $\left(1+\tau_{h}^{I}\right) q^{I}\left(g+\delta_{h}\right) k_{h}$ ersetzen. Folglich ist bereits für das Umsatzsteuersystem an sich zu erkennen, daß zumindest auf die Investitionsnachfrage von umsatzsteuerbefreiten Unternehmen faktisch ein Investitionssteuersatz angewandt wird, der sich als gewichtetes arithmetisches Mittel der nominellen Umsatzsteuersätze darstellt.

Fassen wir die Produzentenpreis-Bestimmungsgleichungen abzugsberechtigter und befreiter Unternehmen zusammen, erhalten wir in Matrixform

$$
\begin{aligned}
q & =\left(A^{\prime}+A^{\prime}{ }_{m} \hat{\tau}\right) q+\left(B^{\prime}+B^{\prime}{ }_{m} \hat{\tau}\right) q+W_{z} \\
& =\left(I-A^{\prime}-A^{\prime}{ }_{m} \hat{\tau}-B^{\prime}-B^{\prime}{ }_{m} \hat{\tau}\right)^{-1} W_{z} .
\end{aligned}
$$

Ein typisches Element der Matrix $B^{\prime}$ wäre zum Beispiel $[B]_{i j}=$ $\beta_{j}\left(g+\delta_{i}\right) k_{i}$. In denjenigen Zeilen, die für befreite Unternehmen relevant sind, weist die Matrix $B^{\prime}{ }_{m}$ dieselben Elemente wie $B^{\prime}$ auf, alle anderen Elemente sind gleich Null. $W_{z}$ bezeichnet schließlich einen Spaltenvektor, der sich aus den Elementen $r z_{i}-\dot{z}_{i}+w l_{i}$ zusammensetzt.

Zur Feststellung der impliziten Steuerbeträge pro Besteuerungseinheit $\tilde{t}_{i}$ ziehen wir wiederum die fiktiven Produzentenpreise $\tilde{q}_{i}$ heran, die sich ohne (direkten) Einfluß der Steuersätze einstellen würden. Sie werden über

$$
\tilde{q}=\left(I-A^{\prime}-B^{\prime}\right)^{-1} W_{z}
$$

bestimmt. Aus der Differenz von $q$ und $\tilde{q}$ erhalten wir 


$$
t:=q-\tilde{q}=\left(I-A^{\prime}-B^{\prime}\right)^{-1}\left(A^{\prime}{ }_{m}+B^{\prime}{ }_{m}\right) \hat{\tau} q .
$$

Die effektiven Konsumsteuersätze folgen unmittelbar aus den in Teilabschnitt (4.1) getätigten Definitionen. Zur Wiederholung: Aus $\tilde{t}_{i}$ gewinnt man über $\tilde{t}_{i} / \tilde{q}_{i}$ die impliziten Steuersätze $\tilde{\tau}_{i}$, woraus sich die effektiven Konsumsteuersätze als $\tau_{i}^{c}=\tau_{i}^{u}+\tau_{i}^{u} \tilde{\tau}_{i}+\tilde{\tau}_{i}$ ergeben.

Da bereits für das Umsatzsteuersystem eine Art Investitionssteuer für befreite Unternehmen festgestellt wurde, ist es nicht schwer einzusehen, da $\beta$ diese Steuer auch im äquivalenten Steuersystem erhoben werden muß. Legt man anstelle der $q_{i}$ die fiktiven Produzentenpreise $\tilde{q}_{i}$ als Basis für den Nettopreis der Investitionen $\tilde{q}^{I}$ zugrunde, erhalten wir die effektiven Investitionssteuersätze $\tau_{h}^{I}$ nun durch

$$
\tau_{h}^{I}:=\frac{t^{I}}{\tilde{q}^{I}}=\frac{\sum_{j} \beta_{j}\left(\tau_{j}^{u}+\tau_{j}^{u} \tilde{\tau}_{j}+\tilde{\tau}_{j}\right) \tilde{q}_{j}}{\sum_{j} \beta_{j} \tilde{q}_{j}} \quad h=n-m+1, \ldots, n .
$$

Damit ist aber der durch $t_{i}$ bzw. $\tilde{\tau}_{i}$ charakterisierte Teil des Steueraufkommens im alternativen Steuersystem noch nicht vollständig erfaßt. Erst durch die Besteuerung der Investitionen abzugsberechtigter Unternehmen mit einem Steuersatz in Höhe von

$$
\tau_{i}^{I}=\frac{\sum_{j} \beta_{j} \tilde{\tau}_{j} \tilde{q}_{j}}{\sum_{j} \beta_{j} \tilde{q}_{j}} \quad i=1, \ldots, n-m
$$

wird das Steueraufkommen des Umsatzsteuer-Gleichgewichts erreicht. Die Steuersätze gewährleisten auch, daß die Kapitalnachfrage unter beiden Steuersystemen gleich hoch ist. Zur Bestätigung dieser Behauptung muß keine explizite Nachfragefunktion aus dem Optimierungskalkül der Unternehmen abgeleitet werden; eine einfache ArbitrageÜberlegung genügt. Eine marginale Einheit Kapital erwirtschaftet den Ertrag $\left(\partial f_{i} / \partial K_{i}\right)\left(q_{i}-\sum_{j} a_{j i} q_{j}\right)$. Nach Ablauf der Produktionsperiode hat sich jedoch der Wert dieser Einheit um $\delta_{i} q^{I}$ reduziert, und es sind Dividendenzahlungen in Höhe $r q^{I}$ auf die marginale Wertpapieremission angefallen. Im Umsatzsteuer-Gleichgewicht wird ein abzugsberechtigtes Unternehmen $i$ folglich in dem Umfang Kapital nachfragen, 
bis die Optimalitätsbedingung $\left(\partial f_{i} / \partial K_{i}\right)\left(q_{i}-\sum_{j} a_{j i} q_{j}\right)=\left(r+\delta_{i}\right) q^{I}$ erfüllt ist ${ }^{67}$. Aufgrund der Investitionssteuersätze $\tau_{i}^{I}$ sehen sich die Unternehmen wegen $q_{i}^{I}=\left(1+\tau_{i}^{I}\right) \tilde{q}_{i}^{I}$ derselben Optimalitätsbedingung im äquivalenten Steuersystem gegenübergestellt und werden folglich auch Kapital in gleicher Höhe nachfragen.

Im Modell einer wachsenden Ökonomie ist die Erhebung einer Umsatzsteuer äquivalent zu einer Besteuerung des Konsums und der Investitionen. Die effektiven Investitionssteuersätze wurden hierbei auf die von den Unternehmen neu erworbenen Kapitalgüter bezogen und nicht auf die Investitionsgüter, aus denen sich Kapital zusammensetzt. Da für alle Unternehmen eine einheitliche Transformationsfunktion unterstellt wurde, die die einzelnen Investitionsgüter in das Kapitalgut umwandelt, gibt es nur zwei verschiedene effektive Investitionssteuersätze. Auf Investitionen von Unternehmen, die im UmsatzsteuerGleichgewicht steuerbefreit sind, wird im äquivalenten Steuersystem der effektive Steuersatz $\tau_{n-m+1}^{I}=\ldots=\tau_{n}^{I}=\tau_{n a}^{I}$ erhoben, auf Investitionen der im Umsatzsteuer-Gleichgewicht vorsteuerabzugsberechtigten Unternehmen der Steuersatz $\tau_{1}^{I}=\ldots=\tau_{n-m}^{I}=\tau_{a}^{I}$. Würde man dagegen unternehmensspezifische Investitionstechnologien unterstellen, dann böten auch die effektiven Investitionssteuersätze kein derart einheitliches Bild mehr. Aber bereits aus unserer einfacheren Variante folgt, daß die Umsatzsteuer nicht nur die Investitionsentscheidung der befreiten und vom Abzug der Vorsteuer ausgeschlossenen Unternehmen direkt beeinflußt, sondern daß die Investitionsentscheidungen aller Unternehmen betroffen sind. Aufgrund der in den Produzentenpreisen implizit enthaltenen Steuerbeträge wirkt die Umsatzsteuer ,unsichtbar" auch auf die für abzugsberechtigte Unternehmen relevanten Optimalitätsbedingungen für effizienten Kapitaleinsatz. In diesem Sinne zerstören unechte Befreiungen die Investitionsneutralität der Umsatzsteuer. Da Befreiungen tendenziell zu Preissteigerungen führen, ist das Investitionsvolumen innerhalb eines solchen Umsatzsteuersystems niedriger als bei einer ausschließlichen Besteuerung des Konsums.

$67 \mathrm{Vgl}$. hierzu auch JORGENSON [1973], S. 542. Es ist jedoch zu beachten, daß das Grenzprodukt $\left(\partial f_{i} / \partial K_{i}\right)$ unter Berücksichtigung von Produktionsverflechtung nicht auf den Güterpreis $q_{i}$, sondern nur auf die Wertschöpfung $\left(q_{i}-\sum_{j} a_{j i} q_{j}\right)$ bezogen wird. 


\section{Die effektiven Steuersätze aus der Datenbasis}

Mit den in Abschnitt (4) präsentierten Berechnungsmethoden sind wir nun in der Lage, aus einer beliebigen - entsprechend aufbereiteten Input-Output-Tabelle effektive Steuersätze aus den nominellen Umsatzsteuersätzen abzuleiten. Zum Abschluß des Kapitels wollen wir die effektiven Steuersätze betrachten, die sich aus der Datenbasis des später verwendeten empirischen allgemeinen Gleichgewichtmodells ergeben. Da die Datenbasis im wesentlichen mit der Input-Output-Tabelle des Statistischen Bundesamtes für das Jahr 1984 übereinstimmt, sind die in Tabelle 4 aufgeführten effektiven Steuersätze um einiges realistischer einzustufen als diejenigen, die aus unserem kleinen Zahlenbeispiel hervorgingen.

Mit der Erstellung einer mikroökonomisch konsistenten Datenbasis werden wir uns in Kapitel III, Teil B ausführlicher befassen. Hier sei nur erwähnt, daß spezifische Charakteristika des unterstellten theoretischen Modells - wie etwa die Annahme eines ausgeglichenen Budgets des Staates ohne Berücksichtigung der Netto-Kreditaufnahme, eine ausgeglichene Leistungsbilanz usw. - Anpassungen der Ausgangsdaten erforderlich macht. Einige dieser Modifikationen sind auch zur Berechnung der effektiven Steuersätze notwendig und werden deshalb kurz problematisiert. Dabei handelt es sich um den Übergang von den statistischen Darstellungseinheiten einer Input-Output-Tabelle zu unseren Modellsektoren $1, \ldots, n$ und der Bildung von nominellen Durchschnittsteuersätzen ${ }^{68}$ für diese Sektoren. Beide sind eng miteinander verbunden.

Die Input-Output-Tabelle des Statistischen Bundesamtes umfaßt 56 homogene Produktionsbereiche. Bei der Tabellenerstellung achtet das Statistische Bundesamt darauf, daß alle Güter eines Bereichs mit einer mehr oder minder einheitlichen Technologie (Input-Struktur) produziert werden und einem ähnlichen Verwendungszweck dienen. Die

${ }^{68}$ Der Begriff nomineller Durchschnittsteuersatz ist noch zu erläutern; an dieser Stelle sei nur darauf hingewiesen, daß er eine aufgrund des Datenmaterials notwendige Hilfskonstruktion umschreibt und nicht auf bestimmte Gesetzesregelungen wie $\S 23$ UstG: Allgemeine Durchschnittsätze oder $\S 24$ UstG: Durchschnittsätze für land- und forstwirtschaftliche Betriebe abzielt. 
Umsatzsteuersätze, die auf die einzelnen Güter anzuwenden sind, bilden allerdings kein Abgrenzungskriterium. Zwar sorgt die Homogenität hinsichtlich der Güterverwendung in den meisten Fällen dafür, daß die Umsätze der verschiedenen Gütergruppen, die zu einem Produktionsbereich zusammengefaßt werden, in der Regel auch einem einheitlichen Steuersatz unterliegen, bei fast allen Produktionsbereichen ist aber auch mindestens eine Ausnahme zu dieser Regel zu finden. Zum Beispiel sind Bahnfahrten, die eine Strecke von $50 \mathrm{~km}$ nicht übersteigen, mit dem ermäßigten Steuersatz zu besteuern ( $\$ 12$ Nr.10bb UStG), während bei längeren Beförderungsstrecken der normale Steuersatz zur Anwendung kommt. Strenggenommen müßten deshalb Bahnfahrten als Leistungen zweier unterschiedlicher Sektoren behandelt werden. Da jedoch keine besonderen Eigenschaften mit der Höhe der Steuersätze verbunden sind, erscheint es durchaus legitim, für die Produktionsbereiche bzw. den daraus resultierenden Sektoren mittlere Steuersätze anzunehmen. Diese nominellen Durchschnittsteuersätze sind so zu bestimmen, daß sie, auf die Umsätze der Produktionsbereiche erhoben, zum selben Steueraufkommen führen wie die nominellen Umsatzsteuersätze angewendet auf die einzelnen Güter bzw. Gütergruppen.

Von zentraler Bedeutung ist dagegen, daß steuerbefreite Güterarten, die nicht zum Abzug der Vorsteuer berechtigen, gesondert ausgewiesen werden. In der Input-Output-Tabelle gibt es aber drei Produktionsbereiche (Produktionsbereich 53-55), die gleichermaßen befreite wie besteuerte Gütergruppen enthalten. Vor der Berechnung effektiver Steuersätze müssen die befreiten von den besteuerten Gütern in irgendeiner Form getrennt werden. Nach welchen Kriterien die Trennung erfolgt, wie die Zusammenfassung der 56 Produktionsbereiche in 15 Sektoren vorgenommen wird, und welche Maßnahmen zur Bestimmung der nominellen Durchschnittsteuersätze im einzelnen notwendig sind, kann aus Kapitel III, Teil B, Abschnitt (2) entnommen werden.

In Tabelle 4 sind nun die nominellen Durchschnittsteuersätze den effektiven Steuersätzen gegenübergestellt ${ }^{69}$. Offensichtlich fallen die

${ }^{69}$ Alle effektiven Steuersätze - einschließlich die Exportsteuer- bzw. Importsubventionssätze - gehen prinzipiell aus Gleichung (27) hervor, obwohl in dem maßgeblichen Teilabschnitt (4.4) Außenhandel nicht explizit modelliert wurde. Wir sahen jedoch im vorangehenden Teil, daß die Berücksichtigung von Außen- 
Tabelle 4: Die effektiven Steuersätze der Datenbasis (in v. H.)

\begin{tabular}{|c|c|c|c|c|c|c|}
\hline Nr. & Sektorbezeichnung & $\tau_{i}^{u}$ & $\tau_{i}^{c *)}$ & $\tau_{i}^{X *)}$ & $\tau_{i}^{M *)}$ & $\tau_{i}^{I *)}$ \\
\hline 1 & Land- und Forstwirtschaft & 7.2 & 7.47 & 0.25 & 0.25 & 0.33 \\
\hline 2 & Energie, Wasser, Bergbau & 13.1 & 13.36 & 0.23 & 0.23 & 0.33 \\
\hline 3 & Chemische Erzeugnisse & 12.3 & 12.66 & 0.31 & 0.31 & 0.33 \\
\hline 4 & $\begin{array}{l}\text { Metallerzeugnisse und } \\
\text { Maschinenbau }\end{array}$ & 14 & 14.38 & 0.33 & 0.33 & 0.33 \\
\hline 5 & Fahrzeuge & 14 & 14.34 & 0.30 & 0.30 & 0.33 \\
\hline 6 & $\begin{array}{l}\text { E-technische und feinme- } \\
\text { chanische Erzeugnisse }\end{array}$ & 13.6 & 13.93 & 0.29 & 0.29 & 0.33 \\
\hline 7 & $\begin{array}{l}\text { Holz- und Papierverarbei- } \\
\text { tung }\end{array}$ & 12.5 & 12.83 & 0.29 & 0.29 & 0.33 \\
\hline 8 & Leder und Bekleidung & 14 & 14.36 & 0.32 & 0.32 & 0.33 \\
\hline 9 & $\begin{array}{l}\text { Nahrungsmittel, Getränke, } \\
\text { Tabak }\end{array}$ & 7.5 & 7.82 & 0.30 & 0.29 & 0.33 \\
\hline 10 & $\mathrm{Bau}$ & 14 & 14.32 & 0.28 & 0.28 & 0.33 \\
\hline 11 & Handel und Transport & 12.9 & 13.45 & 0.48 & 0.48 & 0.33 \\
\hline 12 & $\begin{array}{l}\text { sonst. marktbestimmte } \\
\text { Dienstleistungen }\end{array}$ & 10.4 & 10.76 & 0.33 & 0.33 & 0.33 \\
\hline 13 & $\begin{array}{l}\text { Post und priv. Organisa- } \\
\text { tionen ohne Erwerbszweck }\end{array}$ & 0 & 2.01 & 2.01 & 1.97 & 13.99 \\
\hline 14 & $\begin{array}{l}\text { Banken, Versicherungen, } \\
\text { Wohnungsvermietung }\end{array}$ & 0 & 2.71 & 2.71 & 2.64 & 13.99 \\
\hline 15 & Staat & 0 & 4.85 & - & - & 13.99 \\
\hline
\end{tabular}

*) gerundete Werte

„tatsächlichen“ numerischen Abweichungen der nominellen Sätze von

handel keine Veränderung bei Berechnung der impliziten Steuerbeträge pro Besteuerungseinheit bewirkt. Allerdings werden im nachfolgenden Allgemeinen Gleichgewichtsmodell aus Gründen, die im theoretischen Teil A näher erläutert sind, zum einen keine Neuinvestitionen berücksichtigt, so daß die effektiven Steuersätze für einen Wert von $g=0$ berechnet wurden und zum anderen auch Produktionsteuern modelliert, wodurch in der ersten Klammer von Gleichung (27) die Diagonalmatrix der Produktionsteuersätze mit negativem Vorzeichen hinzukommt. 
den effektiven Konsumsteuersätzen relativ gering aus. In den ersten 12 Sektoren sind die Konsumsteuersätze nur rund $4 \%$ höher als die nominellen Umsatzsteuersätze. Lediglich der Konsum von Gütern bzw. Leistungen der letzten drei Sektoren ist mit recht beachtlichen Steuersätzen behaftet. Gemessen an dem zur Berechnung der effektiven Sätze notwendigen Aufwand ein vielleicht etwas enttäuschendes Ergebnis.

Auf der anderen Seite ist jedoch zu bedenken, daß sich hinter diesen Abweichungen eine Reallokation von mehr als 19 Prozent des gesamtwirtschaftlich erzielten Umsatzsteueraufkommens verbirgt. Darüber hinaus zeigt die Tabelle, daß sowohl Außenhandel wie auch Investitionstätigkeiten - entgegen der eigentlichen Konzeption der Umsatzsteuer - nicht unwesentlich beeinträchtigt sind. Vor allem die von befreiten und nicht abzugsberechtigten Sektoren vorgenommenen Investitionen werden mit nahezu $14 \%$ besteuert $^{70}$. Die Höhe des Steuersatzes erklärt sich aus dem relativ großen Anteil von hochbesteuerten Gütern in der zugrundegelegten Investitionstechnologie. Auch bei der Beurteilung der internationalen Wettbewerbsposition anhand der ausgewiesenen Export/Import-Sätze ist der kumulative Effekt, daß die Exportsteuersätze den Preis für Exportgüter tendenziell steigern und gleichzeitig die Importsubventionssätze die Importgüter verbilligen, zu beachten.

Welche Effizienzwirkungen von den effektiven Steuersätzen im einzelnen ausgehen, wird im nun folgenden Teil dieser Arbeit im Rahmen eines numerisch rechenbaren Gleichgewichtsmodells untersucht. Hierzu sind allerdings eine Reihe von Vorarbeiten notwendig, die Gegenstand des unmittelbar anschließenden Kapitels sind.

${ }^{70}$ Es sei nochmals darauf hingewiesen, daß sich die in den ersten vier Spalten aufgelisteten Steuersätze auf die von den Sektoren $1, \ldots, 15$ erzeugten Güter beziehen, während die Investitionsteuersätze jeweils für die Investitionen nachfragenden Sektoren relevant sind. So gibt beispielsweise $\tau_{10}^{I}$ an, daß die Investitionen des Baugewerbes mit einem Steuersatz von 0.33 Prozent belastet sind und nicht, daß die Bauinvestitionen eines (beliebigen) Sektors mit diesem Prozentsatz besteuert werden. 


\title{
Das Empirische Allgemeine Gleichgewichtsmodell
}

\author{
Teil A: Die Modellstruktur
}

Das Wesen der Empirischen Allgemeinen Gleichgewichtsanalyse besteht darin, Theorie und „Empirie“ miteinander zu verbinden. Theorie, wie sie in den Allgemeinen Gleichgewichtsmodellen walrasianischen Typs zum Ausdruck kommt und Empirie, wie sie sich in den großen gesamtwirtschaftlichen Statistiken - den Volkswirtschaftlichen Gesamtrechnungen oder Input-Output-Tabellen - widerspiegelt. Eines der wichtigsten mit dieser Synthese verfolgten Ziele ist es, empirisch gehaltvollere Aussagen zu ermöglichen, als sie aus rein theoretischen Untersuchungen hervorgehen. In theoretischen Analysen eines komplexeren Besteuerungsproblems tritt nicht selten der Fall ein, daß mehrere, in verschiedene Richtungen weisende, allokative Effekte herausgearbeitet werden können. Der resultierende Wohlfahrtseffekt ist hingegen nicht eindeutig bestimmbar, was in Hinblick auf wirtschaftspolitische Empfehlungen eine nicht allzu hilfreiche Erkenntnis darstellt. Deshalb liegt die Idee nahe, die theoretische Analyse mit konkreten Daten zu „unterfüttern“, um auf diese Weise eindeutigere Wirkungsaussagen zu erlauben. Inwieweit diese eindeutigeren Aussagen jedoch ihrerseits von einschränkenden - aber unausweichlichen - Modellannahmen abhängen, werden wir im Verlauf dieses und der anschließenden Kapitel sehen.

Darüber hinaus bietet die Verwendung eines Empirischen Allgemeinen Gleichgewichtsmodells - kurz EAG-Modells - gegenüber der üblichen theoretischen Marginal-Analyse den Vorteil, auch die Effekte von nicht-marginalen Steuersatzänderungen abschätzen zu können. In unserem Zusammenhang wäre beispielsweise ein Übergang von Nullsteuersätzen zu unechten Befreiungen gleichbedeutend mit der Einführung von (nicht unbedingt sehr kleinen) Steuersätzen auf den Input-Verbrauch befreiter Unternehmen. Eine Untersuchung dieser Art 
von Besteuerungsvariation bereitet in EAG-Modellen keine methodischen Schwierigkeiten.

Auf der anderen Seite bedarf die Einsicht, daß aus der Verbindung von Theorie und statistischen Daten sowie den darauf aufbauenden rechnergestützten Simulationen eine ganze Reihe von Problemen erwachsen, wohl keiner besonderen Vorstellungskraft. Die spezifischen Beschränkungen, denen jede einzelne dieser Disziplinen unterliegt, sind nun für alle Bereiche maßgebend. Bei der Formulierung des theoretischen Teils muß berücksichtigt werden, was rechentechnisch handhabbar und statistisch verfügbar ist, bei der Zusammenstellung des Datenmaterials sind wiederum die Implikationen des theoretischen Modells zu beachten. In Figur 9, welche die zur Aufstellung eines EAGModells erforderlichen Arbeitsschritte zeigt, wurden diese wechselseitigen Abhängigkeiten durch Pfeile markiert.

Figur 9: Die Erstellung eines EAG-Modells

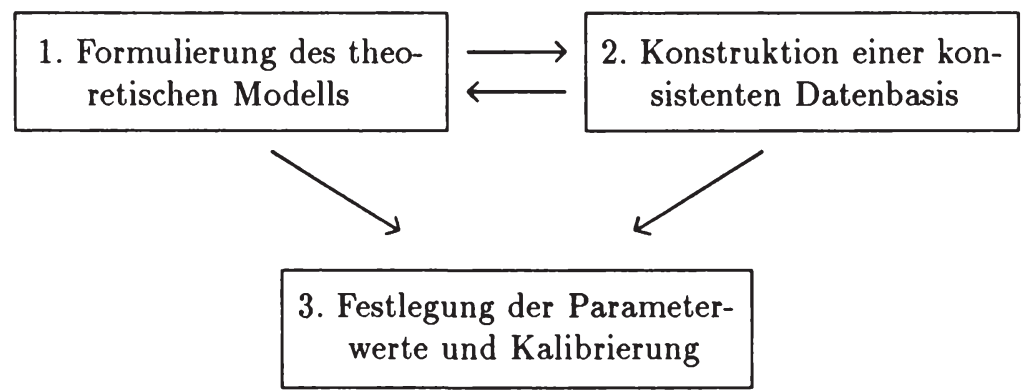

Der vorliegende Teil A des vierten Kapitels ist der Diskussion der funktionalen Modellstruktur gewidmet, die die (noch $\mathrm{zu}$ beschreibenden) Ausgangsdaten als Ergebnis des wirtschaftlichen Handelns aller beteiligten Akteure „erklärt“. Üblicherweise werden hierbei die zu erklärenden Daten als Gleichgewichtswerte aufgefaßt.

Wie in allen Modellen sind auch in dem hier präsentierten die für das jeweilige Erkenntnisobjekt zentralen Aspekte relativ detailliert abgebildet, während weniger wichtig erscheinende Sachverhalte - wenn 
überhaupt - nur in groben Zügen berücksichtigt werden. Gerade in EAG-Modellen mit ihrem Anspruch auf wirtschaftspolitische Relevanz ist die Entscheidung, welche Gegebenheiten in Zusammenhang mit dem $\mathrm{zu}$ untersuchenden Problem wichtig und welche weniger bedeutsam sind, nicht unstrittig. An den betreffenden Stellen werden wir uns mit diesem Gesichtspunkt näher befassen.

Der zweite, in Teil B dargestellte, Arbeitsschritt beinhaltet die Zusammenstellung eines zum theoretischen Modell konsistenten Datensatzes. Er soll das Ausgangsgleichgewicht oder Benchmark Equilibrium numerisch beschreiben. Der Begriff Konsistenz trägt dabei nicht nur dem Umstand Rechnung, daß alle von theoretischer Seite geforderten Gleichgewichtsbeziehungen auch von den Daten einzuhalten sind, sondern daß auch der Aussagegehalt der verwendeten Daten weitgehend mit der theoretischen Bedeutung der modellierten Beziehungen übereinstimmen sollte.

Mit der Konstruktion eines Ausgangsgleichgewichts ist jedoch die numerische Seite noch nicht abgeschlossen. Im dritten und letzten Schritt sind für die exogenen Parameter der unterstellten oder abgeleiteten Verhaltensgleichungen spezifische Werte einzusetzen. Sie werden teils aus der ökonometrischen Literatur entlehnt, teils „kalibriert“. Dieses Verfahren sowie die damit verbundene Problematik wird schließlich in Teil C erläutert.

Die für unsere Zielsetzung wesentlichen Modellelemente sind weitgehend bereits aus Kapitel II bekannt. So befassen wir uns hier hauptsächlich mit der Konkretisierung der im zweiten Kapitel allgemein gehaltenen funktionalen Beziehungen und der dabei zu beachtenden Punkte. Als einzige Modellerweiterung werden wir zwei weitere, für den Unternehmensbereich relevante Steuer arten - Produktion- und Faktorsteuern - berücksichtigen und dem von Sektor 15 produzierten Gut eine besondere Bedeutung zuweisen. 


\section{Der repräsentative Haushalt}

Bevor wir uns mit der Auswahl eines bestimmten Funktionstyps für die Nutzenfunktion des repräsentativen Haushalts auseinandersetzen, sei auf eine nicht ganz unproblematische Modelleinschränkung aufmerksam gemacht. Von allen möglichen ökonomischen Entscheidungen, die der Haushalt zu treffen hat, wird nur die Wahl der Konsumgüter und seine Freizeitnachfrage modelliert. Die Bildung von Ersparnissen bleibt dagegen unberücksichtigt. Zur Begründung dieser einschneidenden Maßnahme müssen wir etwas weiter ausholen. Üblicherweise wird die Ersparnisbildung in EAG-Modellen über die Erweiterung der Konsummöglichkeiten in zukünftigen Perioden motiviert, wobei sich die Höhe der Ersparnisbildung u. a. an den erwarteten Preisen - in der Regel unterstellt man dabei myopische oder adaptive Erwartungen ${ }^{71}$ - für zukünftige Konsumgüter orientiert. Mit der Höhe der Ersparnisse liegt über die Gleichgewichtsbedingung für den Finanzierungsmarkt auch die Höhe des Investitionsvolumens fest. Eine gesonderte Modellierung der Investitionsnachfrage der Unternehmen unterbleibt jedoch. Zwar wäre durch diese Vorgehensweise ein möglicher Einfluß der Umsatzsteuer auf die gleichgewichtigen Ersparnisse - via Preiserwartungen für zukünftige Konsumgüter - in das Modell implementierbar, eine derartige Behandlung der unternehmerischen Investitionsentscheidung wird unserer Zielsetzung jedoch nicht gerecht, zumal die Umsatzsteuersätze die Investitionsentscheidung befreiter Unternehmen direkt beeinflussen. Der Grund, warum die Ersparnisbildung nun gänzlich vernachlässigt wird, ist deshalb eher in der schwierigen Implementierung einer problemgerechten Investitionsnachfrage zu sehen. Wir werden diesen Punkt bei der Beschreibung des Unternehmerverhaltens nochmals aufgreifen.

Die Auswahl eines bestimmten funktionalen Typs für unsere Nutzenfunktion $U=U\left(C_{1}, \ldots, C_{14}, F\right)$ sollte in EAG-Modellen zwei Eigenschaften genügen. Sie sollte erstens nicht allzu schwierig zu handhaben sein, aber zweitens dennoch eine ausreichende Flexibilität bezüglich des

${ }^{71}$ Siehe bspw. Fullerton/Shoven/Whalley [1983] oder Ballard und GOULDER [1985] für eine Wirkungsanalyse verschiedener Annahmen bezüglich der Erwartungsbildung. 
Tabelle 5: Ausgewählte Elastizitäten für alternative Nutzenfunktionen

\begin{tabular}{|c|c|c|c|}
\hline Nutzenfunktion & Cobb-Dgl. & CES & Stone-Geary \\
\hline $\begin{array}{l}\text { funktionale } \\
\text { Form } C\end{array}$ & $\prod_{i} C_{i}^{\alpha_{i}}$ & {$\left[\sum_{i} \theta_{i}^{\frac{1}{\nu}} C_{i}^{\frac{\nu-1}{\nu}}\right]^{\frac{\nu}{\nu-1}}$} & $\prod_{i}\left(C_{i}-\gamma_{i}\right)^{\alpha_{i}}$ \\
\hline mit & $\begin{array}{c}\alpha_{i}>0 \\
\sum_{i} \alpha_{i}=1\end{array}$ & $\begin{array}{c}\theta_{i}>0 \\
\sum_{i} \theta_{i}^{\frac{1}{\nu}}=1\end{array}$ & $\begin{array}{c}\alpha_{i}>0 \\
\sum_{i} \alpha_{i}=1\end{array}$ \\
\hline $\begin{array}{l}\text { Nachfrage- } \\
\text { funktion } C_{i}\end{array}$ & $\frac{\alpha_{i} Y}{p_{i}}$ & $\frac{\theta_{i} Y}{p_{i}^{\nu} \sum_{j} \theta_{j} p_{j}^{1-\nu}}$ & $\gamma_{i}+\frac{\alpha_{i}(Y-\Gamma)}{p_{i}}$ \\
\hline $\begin{array}{l}\text { direkte Preis- } \\
\text { elastizität } \epsilon_{i i}\end{array}$ & -1 & $-\nu-(1-\nu) \frac{p_{i} C_{i}}{Y}$ & $-1+\frac{\left(1-\alpha_{i}\right) p_{i} \gamma_{i}}{p_{i} C_{i}}$ \\
\hline $\begin{array}{l}\text { Kreuzpreis- } \\
\text { elastizität } \epsilon_{i k}\end{array}$ & 0 & $-(1-\nu) \frac{p_{k} C_{k}}{Y}$ & $\frac{-\alpha_{i} p_{k} \gamma_{k}}{p_{i} C_{i}}$ \\
\hline $\begin{array}{l}\text { Einkommens- } \\
\text { elastizität } \epsilon_{i Y}\end{array}$ & 1 & 1 & $\frac{\alpha_{i} Y}{p_{i} C_{i}}$ \\
\hline
\end{tabular}

Quelle: GotTFRIEd/STöss/WIEgARD [1990], S. 209.

daraus abgeleiteten Verhaltens des Haushalts gestatten. Dabei denkt man sofort an Cobb-Douglas-, CES- oder LES-Funktionen. Auf der anderen Seite sind mit diesen Funktionstypen eher unattraktive empirische Implikationen verbunden ${ }^{72}$. Zum Beispiel weisen die aus einer

${ }^{72}$ Vgl. hierzu auch DEATON [1974]. 
Cobb-Douglass-Nutzenfunktion abgeleiteten Nachfragefunktionen alle eine Kreuzpreiselastizität von Null auf. Einige dieser empirischen Implikationen sind für die oben genannten Funktionstypen - jeweils am Beispiel einer über die Konsumgüter $C_{i}$ definierten Nutzenfunktion in Tabelle 5 zusammengestellt.

Etwas größere Flexibilität erreicht man durch die Konstruktion von sog. Nutzenbäumen ${ }^{73}$. Bei dieser Methode werden die Argumente der Nutzenfunktion nach verschiedenen Merkmalen hierarchisch geordnet, wobei für jede der hierarchischen Ebenen eine gesonderte SubNutzenfunktion bestimmten Typs definiert werden kann. Der aus einer Ebene resultierende Nutzenindex geht dann als Argument - das eine Art Composite-Commodity beschreibt - in die Sub-Nutzenfunktion der unmittelbar übergeordneten Ebene ein. So entsteht ausgehend von der obersten Ebene eine Baumstruktur, bei der die einzelnen „Verästelungen“ jeweils durch einen anderen Funktionstyp gekennzeichnet sein können.

Neben der recht komfortablen Möglichkeit mittels Kombination relativ einfacher funktionaler Formen eine etwas komplexere GesamtNutzenfunktion zu erhalten, besitzt dieses Verfahren ferner die angenehme Eigenschaft, daß unter bestimmten Voraussetzungen die Nutzenmaximierung des Haushalts als mehrstufiges Optimierungsproblem aufgefaßt werden kann ${ }^{74}$. In Figur 10 ist zunächst jedoch die Baumstruktur der Nutzenfunktion unseres repräsentativen Haushalts angegeben. Die Nutzenfunktion ist additiv separabel zwischen der Nutzenstiftung aus Güterkonsum und Freizeit $U(C, F)$ und der Nutzenstiftung des öffentlichen Gutes $H(G)$. Für die erste Ebene wurde eine CESFunktion gewählt, die über das Composite-Commodity Güterkonsum und die Freizeit definiert ist, für die zweite Ebene eine Cobb-DouglasFunktion, welche die Konsumgüter $C_{1}, \ldots, C_{14}$ in das CompositeCommodity $C$ zusammenfaßt ${ }^{75}$.

\footnotetext{
${ }^{73}$ Siehe hierzu bspw. STrotz [1957,1959] oder KELLER [1976].

${ }^{74}$ Beispielsweise muß hierzu die Nutzenfunktion bezüglich der einzelnen SubNutzenindices schwach separabel sein (vgl. auch BLACKORBY/LADY/NISSEN/RUSSEL [1970]).

${ }^{75}$ Die Nutzenstiftung $H(G)$ wird nicht näher spezifiziert. Da das Bereitstellungsniveau $G$ des Kollektivgutes nicht der Entscheidung des repräsentativen
} 
Figur 10: Struktur des Nutzenbaums

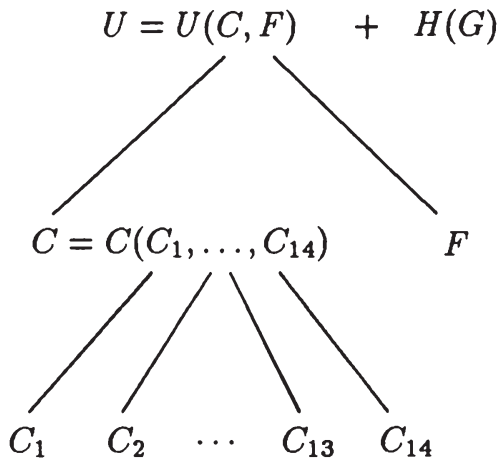

Das Nachfrageverhalten des repräsentativen Haushalts kann nun (fiktiv) aus zwei sukzessiven Entscheidungsstufen abgeleitet werden. Zunächst trifft er entsprechend des Kalküls

$$
\max _{C, F} \quad U=\left[(1-\theta)^{\frac{1}{\nu}} C^{\frac{\nu-1}{\nu}}+\theta^{\frac{1}{\nu}} F^{\frac{\nu-1}{\nu}}\right]^{\frac{\nu}{\nu-1}}
$$

unter der Nebenbedingung: $\quad Y^{m a x}=p C+w F$

seine für ihn optimale Wahl zwischen Konsum- und Freizeitnachfrage. Die Erläuterung des Preisindex $p$ wollen wir etwas zurückstellen, die Bewertung der Freizeit folgt dem Opportunitätskostenprinzip: Ein bestimmtes $\mathrm{Maß}$ an Freizeit bedeutet für den Haushalt gleichzeitig einen Verzicht auf Arbeitseinkommen zum Lohnsatz $w^{76}$. Aus der ersten Entscheidung erhalten wir die Freizeitnachfragefunktion

Haushalts unterliegt und er auch keinen direkten Preis dafür zu zahlen hat, übt die Bereitstellung von $G$ keinen Einfluß auf sein Nachfrageverhalten bezüglich der Konsumgüter und der Freizeit aus.

${ }^{76}$ Zur Erläuterung von $Y^{\max }$ siehe Kapitel II, Abschnitt 1, S. 52. 


$$
\begin{gathered}
F=\frac{\theta(w E+r \bar{K}+T R)}{\Omega w^{\nu}} \\
\text { mit } \quad \Omega=(1-\theta) p^{1-\nu}+\theta w^{1-\nu},
\end{gathered}
$$

die wegen $E-F=L$ auch das Arbeitsangebot des Haushalts determiniert. Nach dieser Entscheidung steht dem Haushalt zur optimalen Konsumgüterwahl nur noch ein Einkommen von $Y^{v e r}=Y^{\max }-w F=$ $r \bar{K}+w L+T R$ zur Verfügung, welches über das anschließende Optimierungsproblem

$$
\begin{array}{cl}
\max _{C_{i}} C=\prod_{i=1}^{14} C_{i}^{\alpha_{i}} & \text { mit } \quad \alpha_{i}>0 ; \sum_{i} \alpha_{i}=1 \\
\text { unter der Nebenbedingung: } & Y^{\text {ver }}=\sum_{i=1}^{14} p_{i} C_{i}
\end{array}
$$

auf die Ausgaben für die einzelnen Konsumgüter aufgeteilt wird. Die aus der zweiten Entscheidung resultierenden Konsumgüter-Nachfragefunktionen können direkt aus Tabelle 5 entnommen werden.

Nun sind wir auch in der Lage, den für die erste Stufe relevanten Preisindex $p$ etwas näher zu erläutern. Setzen wir die KonsumgüterNachfragefunktionen in die zugehörige (direkte) Sub-Nutzenfunktion ein, erhalten wir mit

$$
C=\prod_{i=1}^{14}\left(\frac{\alpha_{i}}{p_{i}} Y^{\text {ver }}\right)^{\alpha_{i}}
$$

die indirekte Nutzenfunktion für diese Ebene. Daraus kann wiederum die Ausgabenfunktion abgeleitet werden, die wegen $\sum_{i} \alpha_{i}=1$ die Form

$$
Y^{v e r}=C \prod_{i=1}^{14}\left(\frac{p_{i}}{\alpha_{i}}\right)^{\alpha_{i}}
$$

annimmt. Der Preisindex $p$ ist folglich durch 


$$
p:=\prod_{i=1}^{14}\left(\frac{p_{i}}{\alpha_{i}}\right)^{\alpha_{i}}
$$

definiert. Er repräsentiert gewissermaßen den Preis, der für die Nutzenstiftung $C$ aus Güterkonsum zu zahlen ist.

Damit sind die Nachfragefunktionen des repräsentativen Haushalts spezifiziert. Gleichzeitig hat die Verwendung von Nutzenbäumen gezeigt, wie auf relativ unkomplizierte Weise die Flexibilität einfacher funktionaler Typen erhöht werden kann, ohne allzu komplexe Umformungen in Kauf nehmen zu müssen. In dem mehrstufigen Entscheidungsverfahren sind auf jeder Stufe nur die zugehörigen Preise (bzw. Preisindices) und Einkommensanteile relevant. Die Preise anderer Verästelungen üben - über die Konstruktion des entsprechenden Preisindex - lediglich einen mittelbaren Einfluß aus.

Abschließend sei jedoch angemerkt, daß die restriktiven empirischen Eigenschaften der auf den einzelnen hierarchischen Ebenen angewandten Funktionstypen zwar nicht mehr für die gesamte Nutzenfuktion, aber dennoch für die jeweilige Ebene ausschlaggebend sind. Bedenkt man, in welchem Maß die Ausgestaltung eines Steuersystems von der Präferenzordnung abhängt ${ }^{77}$, wird deutlich, daß über die Wahl einer funktionalen Form für die Nutzenfunktion zum Teil auch die Modellergebnisse vorbestimmt sind. Eine Alternative, die diese restriktiven Implikationen größtenteils überwindet, bietet die Verwendung sog. flexibler funktionaler Formen - wie etwa Translog-Funktionen - zur Beschreibung der indirekten Nutzenfunktion ${ }^{78}$. Da auf der anderen Seite für diesen Ansatz ein beträchtlich umfangreicherer Datensatz benötigt wird, kam er bislang nur bei einer Arbeitsgruppe um JoRGENSEN [1983,1984] zur Anwendung.

\footnotetext{
${ }^{77} \mathrm{Vgl}$. hierzu auch DEATON [1981].

${ }^{78}$ Für eine nähere Beschreibung sowie der damit verbundenen Vor- und Nachteile siehe auch Deaton/Muellbauer [1980], S. 73 f. oder Diewert [1985].
} 


\section{Die Unternehmen}

Der Unternehmensbereich ist in 15 Sektoren unterteilt, wobei dem von Sektor 15 hergestellten Gut (Leistungen des Staates) eine Sonderstellung zukommt. Es kann entweder als Zwischenprodukt verwendet werden, oder es wird von staatlicher Seite nachgefragt und als Kollektivgut $G$ der Allgemeinheit ohne spezielles Entgelt zur Verfügung gestellt. Die Bezeichnung des Sektors 15 als Staat charakterisiert somit nur bedingt den modellierten Sachverhalt. Modelltechnisch gesehen produziert auch Sektor 15 unter konkurrenzwirtschaftlichen Bedingungen. Lediglich dem Outputanteil, der in die Endnachfrage gelangt, werden die Merkmale eines öffentlichen Gutes zugewiesen. Diese Behandlung von Sektor 15 erklärt sich hauptsächlich aus der vorgegebenen Datensituation. Auch in Input-Output-Tabellen sind bspw. die Gebietskörperschaften als Produktionsbereich dargestellt, wobei (im Jahr 1984) rund $19 \%$ des Bruttoproduktionswerts als Vorleistungen verbraucht wurden.

Die unterstellten Produktionsfunktionen sind bereits aus Kapitel II, Abschnitt (1) bekannt. Vor der Erläuterung des unternehmerischen Optimierungsproblems und der Ableitung der Faktornachfragefunktionen wollen wir jedoch nochmals auf die Frage der Behandlung von Investitionen im Rahmen von EAG-Modellen zurückkommen und - im Anschluß daran - die Vorgehensweise bei der im Unternehmensbereich nun zusätzlich berücksichtigten Steuerarten etwas näher diskutieren.

\subsection{Investitionen}

Neben der im vorangehenden Abschnitt angedeuteten Methode, die Investitionen über die Ersparnisbildung des privaten Haushalts zu integrieren, während die eigentliche Faktoreinsatzentscheidung der Unternehmen auf einem statischen Kalkül basiert, wurde in jüngerer Zeit - mit den Modellen von bspw. Auerbach/Kotlikoff [1987], Goulder/Summers [1987] oder Pereira [1988] - auch ein explizites dynamisches Unternehmerverhalten in EAG-Modelle eingeführt. In der Regel maximieren die Unternehmer dabei den Gegenwartswert ihrer Firma unter der Restriktion, daß eine Veränderung des Kapitalstocks Anpassungskosten verursacht (vgl. LuCAS [1967]). Die (Netto-) Investitionsnachfrage ergibt sich dann in jeder Periode endogen aus der 
Differenz zwischen dem tatsächlichen Kapitalstock des Unternehmens und dem abgeleiteten optimalen Kapitaleinsatz.

Die Implementation dieses dynamischen Unternehmerverhaltens in EAG-Modelle hat jedoch auch ihre Schattenseite. Bei statischer Betrachtungsweise hängen die Produzentenpreise - aufgrund der NullProfit-Bedingungen - nur von den Faktorpreisen für Arbeit und Kapital (und ggf. bestimmten Steuersätzen) ab. Für Simulationsrechnungen muß folglich nur ein gleichgewichtiger Faktorpreisvektor gefunden werden, der dann seinerseits die gleichgewichtigen Produzenten- bzw. Konsumentenpreise determiniert. Unterstellt man dagegen - wie es bei der Maximierung des Gegenwartswerts eines Unternehmens der Fall ist einen pro Periode fixen, langfristig jedoch veränderlichen Kapitalstock, sind (optimale) positive Profite in einer Periode nicht auszuschließen. Die Dimension des zu suchenden Gleichgewichts-Preisvektors kann deshalb nicht auf die Anzahl der Primärfaktoren reduziert werden, sondern sie entspricht (mindestens) der Anzahl der Güter- und Faktorpreise. Um die aufzuwendende Rechnerzeit in einigermaßen vertretbaren Grenzen zu halten, wird daher der Unternehmensbereich nur in sehr wenige - typischerweise zwei - Sektoren (und somit Güter) unterteilt. Für die Untersuchung der Wohlfahrtseffekte, die von alternativen Umsatzsteuerregelungen - insbesondere von unechten Befreiungen - ausgehen, wäre eine Beschränkung auf zwei Sektoren jedoch nicht angemessen.

Um andererseits die allokativen Effekte, welche die Umsatzsteuer auf den Kapitaleinsatz der Unternehmen ausüben kann, nicht gänzlich zu ignorieren, wollen wir eine Art Kompromißweg zwischen der kompletten Vernachlässigung von Investitionen und ihrer vollständigen Integration beschreiten: Der gesamtwirtschaftlich konstante Kapitalstock $\bar{K}$ befinde sich im Besitz des repräsentativen Haushalts, der ihn periodenweise an die Unternehmen vermietet. Im Laufe der Periode unterliegt der Kapitaleinsatz einem produktionsbedingten Verschleiß - den ökonomischen Abschreibungen. Am Ende der Periode muß der eingesetzte Kapitalstock jedoch in voller Höhe an den Haushalt zurückerstattet werden. Auf diese Weise ist es möglich, zumindest Reinvestitionen ohne größeren Aufwand in das Modell einzubeziehen, wobei in den Kapitalnutzungspreis, der die (statische) Kapitaleinsatzentscheidung der Unternehmen mit beeinflußt, auch der Preis für Investitionen einfließt. 
Die Abschreibungen $q^{I} D_{i}$ bilden dabei - wie bereits erläutert - einen sektorspezifischen, aber konstanten Anteil $\delta_{i}$ des eingesetzten Kapitals

$$
D_{i}=\delta_{i} K_{i} . \quad i=1, \ldots, 15
$$

Ebenso wird die in Kapitel II Teilabschnitt (4.4) unterstellte Investitionstechnologie zur Umwandlung der einzelnen Investitionsgüter in die (zu ersetzenden) Kapitaleinheiten

$$
D_{i}=\min \left[\frac{I_{1 i}}{\beta_{1}}, \ldots, \frac{I_{14 i}}{\beta_{14}}\right]
$$

verwendet. Ohne Berücksichtigung von Steuern muß nun Unternehmen $i$ für den Einsatz von $K_{i}$ Kapitaleinheiten mit Kosten in Höhe von $r K_{i}+q^{I} D_{i}$ kalkulieren.

\subsection{Produktion- und Faktorsteuern}

Mit der Einführung von Produktion- und Faktorsteuern wird dem Umstand Rechnung getragen, daß die allokativen Wirkungen der Umsatzsteuer nicht zuletzt auch von der Ausgestaltung anderer Steuerarten abhängen. Im Unternehmensbereich wäre hier vor allem an die indirekten Steuern oder die Körperschaftsteuer zu denken. Wir wollen diese Steuerarten jedoch nicht in allen ihren technischen Einzelheiten modellieren, sondern sie nur durch ihre jeweils wesentlichen Anknüpfungspunkte typisieren. So unterscheiden wir lediglich zwischen einer Produktionsteuer, die den (wertmäßigen) Unternehmensoutput insgesamt zur Bemessungsgrundlage hat, und einer partiellen Faktorsteuer auf Kapital - im folgenden kurz Kapitalsteuer genannt -, die auch das Faktoreinsatzverhältnis beeinflußt. In diesem Teilabschnitt wird die Zuordnung der verschiedenen Steuerarten zu den beiden Modellsteuern kurz begründet bzw. kritisch beleuchtet.

a) Produktionsteuern

Bis auf eine Ausnahme, sind unter dem Oberbegriff Produktionsteuern dieselben Steuerarten (und Subventionen) subsummiert, die auch in Input-Output-Tabellen des Statistischen Bundesamtes in der Zeile Produktionsteuern abzgl. Subventionen vertreten sind. Dazu gehören 
u. a. die spezifischen Verbrauchsteuern, wie etwa die Branntweinsteuer, die Mineralölsteuer, die Tabaksteuer, etc., aber auch die Realsteuern, die Versicherungsteuer, einige Verwaltungsgebühren und ähnliche Abgaben ${ }^{79}$. Im Gegensatz zum Vorgehen des Statistischen Bundesamtes werden die Realsteuern hier jedoch nicht zu den Produktionsteuern gezählt.

Bereits aus dieser auszugsweisen Aufzählung geht hervor, daß die stellvertretende Modellierung einer nach Sektoren differenzierenden Produktionsteuer wohl kaum jeder einzelnen der Steuerarten gerecht werden kann. Beispielsweise handelt es sich bei der Branntwein- und der Mineralölsteuer um reine Mengensteuern, wobei die Tarife nach qualitativen Merkmalen - bei der Branntweinsteuer darüber hinaus quantitativ - abgestuft sind, dagegen wird die Tabaksteuer teilweise und die Versicherungsteuer zwangsläufig als Wertsteuer erhoben. Ferner kommt bei Verbrauchsteuern das Bestimmungslandprinzip zur Anwendung, um nur einige der Verschiedenheiten zu nennen. Dessen ungeachtet wollen wir all diese Steuerarten als spezifische Wertsteuer behandeln und darüber hinaus auch für die Subventionen eine proportionale Beziehung zu dem entsprechenden Unternehmensoutput unterstellen. Letztere können somit in die Produktionsteuer aufgenommen werden, so daß der Steuerbetrag

$$
T_{i}^{q}=\tau_{i}^{q} q_{i} Q_{i}
$$

eine Art Differenzgröße zwischen der für Sektor $i$ relevanten Steuern und Subventionen darstellt. Durch die Anwendung des Produktionsteuersatzes $\tau_{i}^{q}$ auf den Bruttoproduktionswert - inklusive der Steuer ersparen wir uns die Einführung eines zusätzlichen Symbols für den Herstellungspreis (= Produzentenpreis - Steuer + Subventionen).

\section{b) Kapitalsteuern}

Als Faktorsteuer auf Kapitaleinsatz werden die Realsteuern sowie die Körperschaft- und die Kapitalertragsteuer aufgefaßt. Unter Realsteuern versteht man hierbei die den Gemeinden obliegende Grund- und Gewerbesteuer. Die Grundsteuer unterscheidet ihrerseits zwischen der

${ }^{79}$ Ein Liste aller vom Statistischen Bundesamt in Produktionsteuern zusammengefaßten Steuerarten kann aus STBA [1988], S. 38 entnommen werden. 
Besteuerung von land- und forstwirtschaftlichen Betrieben (Grundsteuer A) und den sonstigen bebauten und unbebauten Grundstücken (Grundsteuer B). Die Gewerbesteuer ist unterteilt in eine am Gewinn eines Betriebes ansetzende Gewerbeertragsteuer und eine am betrieblichen Einheitswert orientierte Gewerbekapitalsteuer.

In Tabelle 6 ist der Aufbau der Realsteuern skizziert. Sie soll die Grundzüge der jeweiligen Besteuerungsverfahren vermitteln. Die tatsächliche Ausgestaltung der einzelnen Steuern ist allerdings weitaus komplexer als hier dargestellt. Es wäre m. E. auch geeigneter z. B. bei der Gewerbesteuer nicht - wie LiTTMANN - von der Bemessungsgrundlage, sondern von einer ersten Besteuerungsgrundlage zu sprechen.

Genaugenommen geht die Gewerbeertragsteuer von dem nach EStG bzw. KStG ermittelten Gewinn eines Betriebes aus. Zu dem Gewinn werden die Hälfte der Zinsen auf langfristiges Fremdkapital (Dauerschuldzinsen), gezahlte Renten und dauernde Lasten, sofern diese nicht ihrerseits vom Empfänger zur Besteuerung des Gewerbeertrags heranzuziehen sind, die Gewinnanteile eines stillen Gesellschafters unter derselben Einschränkung, die Hälfte der Miet- und Pachtaufwendungen und schließlich die Verlustanteile an zum Betriebsvermögen zählenden Personengesellschaften hinzuaddiert ( $\$ 8 \mathrm{GewStG}$ ). Der daraus resultierende Betrag kann wiederum um 1,2 v. H. des Einheitswerts des zum Betriebsvermögens des Unternehmens gehörenden Grundbesitzes, der Gewinnanteile an Personengesellschaften, der Hälfte der Miet- und Pachterträge und der Spenden für wissenschaftliche $Z$ wecke gekürzt werden ( $\$ 9$ GewStG). Falls in den vorangegangenen fünf Erhebungszeiträumen ein Gewerbeverlust erwirtschaftet wurde, ist auch dieser, sofern er nicht über einen Gewerbeverlustvortrag berücksichtigt wurde, absetzbar. Nach der Kürzung um einen Freibetrag von maximal $36000 \mathrm{DM}$ ( $\S 11$ Abs. $1 \mathrm{GewStG}$ ), wird auf den verbleibenden auf volle Hundert abgerundeten Betrag anschließend eine Steuermeßzahl von grundsätzlich 5 Prozent angewendet. Dies ergibt den Steuermeßbetrag nach dem Gewerbeertrag.

Der Steuermeßbetrag nach dem Gewebekapital wird in ähnlicher Weise berechnet. Ausgehend vom Einheitswert des gewerblichen Betriebes sind 50 v. H. der 50000 DM übersteigenden Dauerschulden, die Rentenschulden und dauernden Lasten, die Verbindlichkeiten gegenüber stillen Gesellschaftern, die Werte bzw. Teilwerte der nicht in Grundbesitz bestehenden Wirtschaftsgüter hinzuzuad- 
Tabelle 6: Aufbau der Realsteuern

\begin{tabular}{|c|c|c|c|c|}
\hline \multirow{2}{*}{$\begin{array}{l}\text { Gesetz } \\
\begin{array}{l}\text { Teil-Ertrag- } \\
\text { steuer }\end{array}\end{array}$} & \multicolumn{2}{|c|}{ Grundsteuergesetz } & \multicolumn{2}{|c|}{ Gewerbesteuergesetz } \\
\hline & $\begin{array}{l}\text { (Grundsteuer A) } \\
\text { Land- und } \\
\text { Forstwirtschaft }\end{array}$ & $\begin{array}{l}\text { (Grundsteuer B) } \\
\text { Grundstücke }\end{array}$ & $\begin{array}{l}\text { Gewerbeertrag- } \\
\text { steuer }\end{array}$ & $\begin{array}{l}\text { Gewerbekapital- } \\
\text { steuer }\end{array}$ \\
\hline Steuertyp & Soll-Ertragsteuer & Soll-Ertragsteuer & Ist-Ertragsteuer & Soll-Ertragsteuer \\
\hline $\begin{array}{l}\text { Bemessungs- } \\
\text { grundlage }\end{array}$ & $\begin{array}{l}\text { Einheitswert } \\
\text { des Betriebes } \\
\text { (Ertragswert) }\end{array}$ & $\begin{array}{l}\text { Einheitswert } \\
\text { des Grundstücks } \\
\text { (Ertragswert, un- } \\
\text { bebaute Grund- } \\
\text { stücke: Gemei- } \\
\text { ner Wert) }\end{array}$ & Reinertrag & $\begin{array}{l}\text { Einheitswert } \\
\text { des Betriebes } \\
\text { (Gemeiner Wert } \\
\text { oder niedrigerer } \\
\text { Teilwert) }\end{array}$ \\
\hline $\begin{array}{l}\text { Bewertungs- } \\
\text { maßstab }\end{array}$ & $\begin{array}{l}\text { 18facher Reinertrag } \\
\text { des land- und } \\
\text { forstwirtschaftlichen } \\
\text { Betriebes; zuzüglich } \\
\text { Wert Wohnteil }\end{array}$ & $\begin{array}{l}\text { Mehrfaches des } \\
\text { Rohertrags der } \\
\text { Grundstücke }\end{array}$ & & ( Marktwert) \\
\hline
\end{tabular}

Quelle: LITTMANN [1980], S. 574 
dieren. Der Einheitswert der Betriebsgrundstücke, die Beteiligungen an Personengesellschaften, die Werte der nicht in Grundbesitz bestehenden Wirtschaftsgüter und die Beteiligungen an ausländischen Kapitalgesellschaften sind abzuziehen. Danach wird der resultierende Betrag auf volle Tausend abgerundet und ein Freibetrag von maximal $120000 \mathrm{DM}$ berücksichtigt. Den Steuermeßbetrag nach dem Gewerbekapital erhält man schließlich nach der Anwendung einer Steuermeßzahl von in der Regel 2 v. T. Beide Steuermeßbeträge addiert (sofern der Betrieb in einer einzigen Gemeinde angesiedelt ist), liefern den einheitlichen Steuermeßbetrag, auf welchen der Hebesatz der Gemeinde anzuwenden ist. Dies ergibt den endgültigen Gewerbesteuerbetrag.

Alle Realsteuern sind in Tabelle 6 als Ertragsteuern klassifiziert wobei die Bezeichnung Soll-Ertragsteuer zum Ausdruck bringt, daß nicht der tatsächliche Ertrag eines Betriebes, sondern dessen im allgemeinen über ein Ertragswertverfahren ermittelte Ertragsfähigkeit als Besteuerungsgrundlage dient - und setzen am Rohertrag bzw. der Nettowertschöpfung (zu Faktorkosten) eines Betriebes an. War durch die Realsteuern ursprünglich eine Besteuerung aller am Ergebnis des Wirtschaftsprozesses beteiligten Faktoren intendiert, so hat sich mit der Abschaffung der Lohnsummensteuer die Bezugsbasis auf die Wertschöpfung abzüglich der Lohnkosten reduziert.

Betrachtet man die Besteuerungsgrundlagen im einzelnen, ist mit Ausnahme der Gewerbeertragsteuer unschwer zu erkennen, daß sie auch auf die Kapitalausstattung der Betriebe ausgerichtet sind. So orientiert sich bspw. der Einheitswert - dessen Ermittlung von Steuer zu Steuer differiert - bei der Grundsteuer A auch an der Betriebsgröße, der -organisation und den Betriebsmitteln, bei der Grundsteuer B ggf. an der Art und Beschaffenheit der auf dem Grund befindlichen Gebäude $^{80}$. Diese Abhängigkeit der Einheitswerte - und damit der Bemessungsgrundlage - von der Kapitalausstattung läßt es sinnvoll erscheinen, die Soll-Ertragsteuern direkt auf das eingesetzte Kapital zu beziehen. Die Modellierung der einzigen Ist-Ertragsteuer, der Steuer auf den Gewerbeertrag, als Kapitalsteuer ist etwas unproblematischer, da der Reinertrag in unserem Modell durch die Faktorentlohnung für

${ }^{80}$ Für eine nähere Beschreibung siehe ANDREAE [1980] S. 581ff. 
Kapital repräsentiert wird ${ }^{81}$.

Im Gegensatz zur Gewerbeertragsteuer ist die Körperschaftsteuer nur für den Gewinn bestimmter Unternehmensformen, insbesondere Kapitalgesellschaften ${ }^{82}$ anzuwenden. Gerade in jüngerer Zeit wurden vor allem durch SINN [1985] massive Einwände gegen die pauschale Behandlung der Körperschaftsteuer als partielle Faktorsteuer auf Kapital erhoben. In einem dynamischen Gleichgewichtsmodell zeigte er, daß die Körperschaftsteuer unter Umständen zwar die Finanzierung des eingesetzten Kapitals bei den betroffenen Unternehmen verändert, die Marginalbedingung für den optimalen Kapitalbestand jedoch nicht beeinflußt ${ }^{83}$. Da das vorliegende Modell keine alternativen Finanzierungsarten für Investitionen berücksichtigt, erscheint es zulässig, der seit HARBERGER [1962] geläufigen Vorgehensweise zu folgen und die Körperschaftsteuer als Faktorsteuer auf Kapital zu betrachten ${ }^{84}$.

Was vielleicht noch einer Bemerkung bedarf, ist die differenzierte steuerliche Behandlung von ausgeschütteten und einbehaltenen Gewinnen. Während einbehaltene Gewinne einem Steuersatz von 56, 50 oder $8 \mathrm{v}$. H. unterworfen sind, beträgt der Steuersatz auf ausgeschüttete Gewinne 36 Prozent. Darüber hinaus ist die Steuer auf ausgeschüttete Gewinne bei der Einkommensteuer anrechenbar. Weniger problematisch ist hierbei die Existenz von unterschiedlichen Steuersätzen - diese müssen aufgrund der Sektoraggregation und der Zusammenfassung mehrerer Einzelsteuern zu einer Kapitalsteuer in jedem Fall gemittelt werden -, sondern vielmehr der Umstand, daß die Körperschaftsteuer auf ausgeschüttete Gewinne zunächst nicht die Faktoreinsatzentscheidungen im Unternehmensbereich, sondern Entscheidungen im Haushaltsbereich beeinflußt.

${ }^{81}$ Die Diskriminierung des Sachkapitals durch die Realsteuern wurde u. a. auch von GUTTING [1988] in einer sehr viel detaillierteren Formulierung der Steuer- sowie der unternehmerischen Finanzierungsseite nachgewiesen.

82 Die von der Körperschaftsteuer betroffenen Organisationsformen können bspw. aus SCHNEIDER [1980], S. 571 f. entnommen werden.

${ }^{83}$ Siehe auch Stiglitz [1973].

${ }^{84}$ Vgl. auch FUllerton/KING/ShOVEN/WhaLLEY [1981], S. 29 f. 
Derselbe Sachverhalt trifft für die Kapitalertragsteuer zu. Sie bildet prinzipiell einen Teil der Einkommensteuer mit der Besonderheit, daß sie im Quellenabzugsverfahren direkt bei den Unternehmen eingezogen wird. Dementsprechend wäre die Steuer - wie auch die Körperschaftsteuer auf ausgeschüttete Gewinne - eigentlich in der Budgetrestriktion des Haushalts etwa in der Form: $Y=w L+\sum_{i}(1-$ $\left.\tau_{i}^{e}\right) r_{i}^{*} K_{i}$ anzusetzen, wobei die $r_{i}^{*}$ die von den Unternehmen gezahlten Bruttopreise für eingesetztes Kapital und $\tau_{i}^{e}$ die gemittelten Steuersätze darstellen.

Wesentlich ist für unsere Betrachtung jedoch nur, daß vollkommene Konkurrenz auf dem Kapitalmarkt eine einheitliche Netto-Kapitalentlohnung sicherstellt. Die Brutto-Faktorpreise für Kapital werden sich im Gleichgewicht gerade so einpendeln, daß die (dem Haushalt zufließende) Netto-Faktorentlohnung für eine Einheit Kapital in allen Sektoren identisch ist. Nur wenn $\left(1-\tau_{i}^{e}\right) r_{i}^{*}=\left(1-\tau_{j}^{e}\right) r_{j}^{*}$ gilt, ist der Haushalt indifferent in seiner Entscheidung, welchem Sektor er Kapital zur Verfügung stellt. Aus diesem Grund spielt es keine Rolle, ob die Steuer auf den Bruttopreis bezogen wird, oder ob ein entsprechender Steuersatz $\tau_{i}^{k}:=\tau_{i}^{e} /\left(1-\tau_{i}^{e}\right)$ auf den für alle Sektoren identischen Nettopreis $r$ zur Anwendung kommt. Ersetzen wir den obigen Bruttopreis $r_{i}^{*}$ durch $\left(1+\tau_{i}^{k}\right) r$ und die Netto-Kapitalentlohnung durch $r \bar{K}$, so können auch die Körperschaftsteuer auf ausgeschüttete Gewinne und die Kapitalertragsteuer zusammen mit den Realsteuern im Unternehmensbereich modelliert werden.

Sicherlich gehen die in diesem Teilabschnitt besprochenen Steuerarten nur auf einem sehr hohen Abstraktionsniveau in das Modell ein. Da sie jedoch nicht im Mittelpunkt des analytischen Interesses stehen, erscheint eine adäquatere Modellierung nicht notwendig. Bei Variation des Umsatzsteuersystems werden sie jedoch die Reaktionen der Unternehmen und die des repräsentativen Haushalts mit beeinflussen. Von daher soll es genügen, die allokativen Wirkungen dieser Steuern, wenn auch nur grob, so doch einigermaßen treffend, einzubeziehen. 


\subsection{Die Faktornachfragefunktionen}

Während eine Leontief-Technologie den Einsatz von Zwischenprodukten bestimmt, wird die Wertschöpfung $f^{i}\left(K_{i}, L_{i}\right)$ des Unternehmens über eine CES-Funktion abgebildet. Damit haben die Produktionsfunktionen allgemein die Form

$$
\begin{gathered}
Q_{i}=\min \left[\frac{1}{a_{0 i}} f^{i}\left(K_{i}, L_{i}\right), \frac{A_{1 i}}{a_{1 i}}, \ldots, \frac{A_{15 i}}{a_{15 i}}\right] \\
\text { mit } \quad f^{i}\left(K_{i}, L_{i}\right)=\Phi_{i}\left\{\rho_{i} L_{i}^{\frac{\sigma_{i}-1}{\sigma_{i}}}+\left(1-\rho_{i}\right) K_{i}^{\frac{\sigma_{i}-1}{\sigma_{i}}}\right\}^{\frac{\sigma_{i}}{\sigma_{i}-1}}
\end{gathered}
$$

für $i=1, \ldots, 15$, wobei $\Phi_{i}$ und $\rho_{i}$ Effizienz- bzw. Gewichtungsparameter repräsentieren und $\sigma_{i}$ die Substitutionselastizität zwischen Arbeit und Kapital bezeichnet. Die Wertschöpfungsfunktionen sind linear homogen in den Primärfaktoren $K_{i}$ und $L_{i}$. Die Produktionsfunktionen sind ihrerseits linear homogen in allen Inputs.

Aufgrund der limitationalen Produktionsstruktur bezüglich der Vorleistungen können die Unternehmen nur den Einsatz der Primärfaktoren optimieren. Da die Wertschöpfungsfunktion wiederum linear homogen ist, minimieren umsatzsteuerpflichtige und zum Abzug der Vorsteuer berechtigte Unternehmen ihre (variablen) Kosten zu gegebenem Wertschöpfungsniveau $a_{0 i} \overline{Q_{i}}$. Aus dem Optimierungskalkül der steuerpflichtigen Unternehmen

$$
\min _{L_{i}, K_{i}} w L_{i}+\left(1+\tau_{i}^{k}\right) r K_{i}+q^{I} \delta_{i} K_{i} \quad i=1, \ldots, 12
$$$$
\text { unter der Nebenbedingung: } \quad f^{i}\left(K_{i}, L_{i}\right)=a_{0 i} \overline{Q_{i}}
$$

erhält man die Faktornachfragefunktionen 


$$
\begin{aligned}
& L_{i}=a_{0 i} \overline{Q_{i}} \frac{1}{\Phi_{i}}\left\{\rho_{i}+\left(1-\rho_{i}\right)\left[\frac{\rho_{i}\left(\left(1+\tau_{i}^{k}\right) r+\delta_{i} q^{I}\right)}{\left(1-\rho_{i}\right) w}\right]^{1-\sigma_{i}}\right\}^{\frac{\sigma_{i}}{1-\sigma_{i}}} \\
& K_{i}=a_{0 i} \overline{Q_{i}} \frac{1}{\Phi_{i}}\left\{\left(1-\rho_{i}\right)+\rho_{i}\left[\frac{\left(1-\rho_{i}\right) w}{\rho_{i}\left(\left(1+\tau_{i}^{k}\right) r+\delta_{i} q^{I}\right)}\right]^{1-\sigma_{i}}\right\}^{\frac{\sigma_{i}}{1-\sigma_{i}}} .
\end{aligned}
$$

Der für den Laufindex $i$ angegebene Wertebereich $(i=1, \ldots, 12)$ bezieht sich hierbei auf die Umsatzsteuerregelungen im Ausgangsgleichgewicht. Das entsprechende Optimierungskalkül von befreiten und nicht vorsteuerabzugsberechtigten Unternehmen - im Ausgangsgleichgewicht Sektor 13,14 und 15 - sowie die daraus resultierenden Faktornachfragefunktionen stimmen mit den obigen Ausdrücken vollkommen überein, mit der Ausnahme, daß anstelle der Nettopreise für Investitionen $q^{I}\left(=\sum_{i=1}^{14} \beta_{i} q_{i}\right)$ die Bruttopreise $p^{I}$ zu setzen sind.

Unter Berücksichtigung der Kapital- und der Produktionsteuern erhalten die Null-Profit-Bedingungen für alle Unternehmen $i=1, \ldots, 15$ nun die Form

$$
p_{i} Q_{i}=\sum_{j=1}^{15} a_{j i} p_{j} Q_{i}+w L_{i}+r K_{i}+p^{I} \delta_{i} K_{i}+T_{i}^{u}+T_{i}^{k}+T_{i}^{q},
$$

wobei $T_{i}^{k}$ und $T_{i}^{q}$ die zu entrichtenden Kapitalsteuer- bzw. Produktionsteuerbeträge beschreiben.

\section{Außenhandel}

In gewisser Hinsicht durchbricht die Modellierung des Außenhandels den bisherigen Grundsatz, alle dargestellten Zusammenhänge über rationales Verhalten der Wirtschaftsubjekte zu erklären. Wollte man die ausländische Güternachfrage bzw. das -angebot explizit auf die Handlungen ausländischer Unternehmen und Haushalte zurückführen, 
so müßte (mindestens) eine komplette zweite Volkswirtschaft formuliert werden. Da das hierzu benötigte Datenmaterial einschließlich der damit verbundenen Anpassungsprobleme um ein Vielfaches ansteigen würde, unterstellen wir stattdessen ein sog. external closing rule zur Beschreibung der grenzüberschreitenden Güterströme ${ }^{85}$.

Die unterstellten ausländischen Exportnachfrage- und Importangebotsfunktionen

$$
\begin{array}{rlrl}
X_{i} & =\left(\frac{q_{i}}{d}\right)^{\Theta} X_{i}^{0} & -\infty<\Theta \leq-1 \\
M_{i}=\left(\frac{q_{i}}{d}\right)^{\eta} M_{i}^{0} & 0 \leq \eta<\infty, i=1, \ldots, 14
\end{array}
$$

sind durch konstante Nachfrage- bzw. Angebotselastizitäten $\Theta$ und $\eta$ bezüglich der entsprechenden Weltmarktpreise $q_{i} / d$ gekennzeichnet, wobei $d$ als "Wechselkurs" oder Preis der Auslandswährung interpretiert werden $\mathrm{kann}^{86}$. Der Term $d$ ist jedoch eine rein finanzielle Größe, die keinen Einfluß auf das langfristige Gleichgewicht unseres realwirtschaftlichen Modells ausübt. Da weder inländische noch ausländische Geldangebots- und -nachfragefunktionen formuliert werden, bestimmen nur die relativen Güterpreise die Höhe des Außenhandels. Der Wechselkurs dient lediglich als Hilfskonstruktion, um die Nachfrage- und Angebotsfunktionen in Abhängigkeit von inländischen Preisen ausdrücken zu können.

Ein Außenhandels- oder Leistungsbilanz-Gleichgewicht ist gegeben, wenn

$$
\sum_{i=1}^{14} q_{i} X_{i}=\sum_{i=1}^{14} q_{i} M_{i}
$$

${ }^{85}$ Diese Vereinfachung ist keineswegs unüblich. Sie wurde bspw. von BALLARD/FUllerton/Shoven/Whalley [1985], oder HiRTE und WiEGARD [1988] benutzt. Eine ausführliche Erläuterung der mit verschiedenen Versionen von external closing rules verbundenen Implikationen befindet sich in Goulder/Shoven/Whalley [1983]. Explizite Mehr-Länder-Modelle kommen prinzipiell nur bei länderübergreifenden Besteuerungsproblematiken - bspw. Steuerharmonisierung - zur Anwendung.

${ }^{86} \mathrm{Vgl}$. auch BOADWAY/TREDDENICK [1978]. 
erfüllt ist. Setzen wir die Exportnachfragefunktionen (28) und die Importangebotsfunktionen (29) in die Gleichgewichtsbedingung ein, ergibt sich aus

$$
\sum_{i=1}^{14} q_{i}\left(\frac{q_{i}}{d}\right)^{\Theta} X_{i}^{0}=\sum_{i=1}^{14} q_{i}\left(\frac{q_{i}}{d}\right)^{\eta} M_{i}^{0}
$$

nach einigen Umformungen mit

$$
d=\left[\frac{\sum_{i} q_{i}^{\eta+1} M_{i}^{0}}{\sum_{i} q_{i}^{\Theta+1} X_{i}^{0}}\right]^{\frac{1}{\eta-\Theta}} \quad \Theta \neq \eta
$$

eine Bestimmungsgleichung für den Wechselkurs. Ersetzen wir daraufhin in (28) und (29) $d$ durch den obigen Ausdruck, erhalten wir mit

$$
\begin{aligned}
X_{i} & =\left[\frac{\sum_{j} q_{j}^{\Theta+1} X_{j}^{0}}{\sum_{j} q_{j}^{\eta+1} M_{j}^{0}}\right]^{\frac{\Theta}{\eta-\Theta}} q_{i}^{\Theta} X_{i}^{0} \\
M_{i} & =\left[\frac{\sum_{j} q_{j}^{\Theta+1} X_{j}^{0}}{\sum_{j} q_{j}^{\eta+1} M_{j}^{0}}\right]^{\frac{\eta}{\eta-\Theta}} q_{i}^{\eta} M_{i}^{0}
\end{aligned}
$$

die gleichgewichtigen Nachfrage- und Angebotsfunktionen in Abhängigkeit der inländischen Preise allein. Beide Funktionen sind homogen vom Grade Null in den Preisen $q_{i}$ für $i=1, \ldots, 14$.

Die direkten Preiselastizitäten (bezüglich der inländischen Preise) der Funktionen sind ebenfalls konstant und durch

$$
\frac{\partial X_{i}}{\partial q_{i}} \frac{q_{i}}{X_{i}}=\frac{\Theta(1+\eta)}{\eta-\Theta} \quad \text { und } \quad \frac{\partial M_{i}}{\partial q_{i}} \frac{q_{i}}{M_{i}}=-\frac{\eta(1+\Theta)}{\eta-\Theta}
$$

gegeben. Hierbei stellt der für $\Theta$ und $\eta$ angegebene Wertebereich sicher, daß die gleichgewichtige Exportnachfrage des Auslands $X_{i}$ (cet. par.) sinkt, das Importangebot $M_{i}$ hingegen steigt, wenn der Preis $q_{i}$ steigt.

Wie eingangs des Teilabschnittes erwähnt, wird mit der Einführung dieser partialökonomischen Nachfrage- und Angebotsfunktionen das 
Ausmaß und die Richtung der grenzüberschreitenden Güterströme eher beschrieben als erklärt ${ }^{87}$. Insbesondere ist der Sachverhalt, daß Güter sowohl importiert als auch exportiert werden, auf das vorgegebene $\mathrm{Da}$ tenmaterial zurückzuführen und nicht durch die theoretische Modellstruktur begründbar.

\section{Der Staat}

Die Beschreibung der staatlichen Aktivitäten kann relativ knapp gehalten werden, zumal die einzelnen Steuern bereits an anderer Stelle aufgeführt und dort erläutert wurden. Auf der Einnahmeseite des staatlichen Budgets sind die Aufkommen aus Produktionsteuern mit

$$
T^{q}=\sum_{i=1}^{15} T_{i}^{q}=\sum_{i=1}^{15} \tau_{i}^{q} q_{i} Q_{i}
$$

und aus Kapitalsteuern mit

$$
T^{k}=\sum_{i=1}^{15} T_{i}^{k}=\sum_{i=1}^{15} \tau_{i}^{k} r K_{i}
$$

zu verbuchen. Hinzu kommt eine Einfuhrumsatzsteuer in Höhe von

$$
T_{M}^{u}=\sum_{i=1}^{14} \tau_{i}^{u} q_{i} M_{i}
$$

sowie das Aufkommen aus der Umsatzsteuer vom Konsumtyp

$$
T^{u}=\sum_{i=1}^{15} T_{i}^{u},
$$

berechnet nach der ebenfalls bekannten allgemeinen Formel

${ }^{87}$ Obwohl es natürlich auch möglich ist, Rückschlüsse auf die den Funktionen unterliegenden ausländischen Nutzenfunktionen zu ziehen (vgl. JOHNSON [1953] oder GORMAN [1957]). 


$$
T_{i}^{u}=\tau_{i}^{u} q_{i}\left(Q_{i}-X_{i}\right)-\sum_{j=1}^{15} a_{j i} \tau_{j}^{u} q_{j} Q_{i}-\sum_{i=1}^{14} \tau_{j}^{u} q_{j} I_{j i}
$$

wobei im Ausgangsgleichgewicht $T_{i}^{u}=\tau_{i}^{u}=0$ für $i=13,14,15$ gilt. Die restlichen Umsatzsteuersätze, wie auch die Produktion- und Kapitalsteuersätze werden aus den Basisdaten ermittelt.

Durch die Bereitstellung des öffentlichen Gutes $G$ entstehen dem Staat Ausgaben in Höhe von $q_{15} G$. Im Benchmark-Gleichgewicht erfolgt der Ausgleich des staatlichen Budgets über die „Transfers“ $T R$, die durch

$$
T R=T^{q}+T^{k}+T_{M}^{u}+T^{u}-q_{15} G
$$

bestimmt sind. Eventuelle Einnahmeüberschüsse werden dabei lumpsum als Transferzahlung dem Haushalt übertragen, eventuelle Defizite ebenfalls lump-sum als „Einkommensteuer“ erhoben. 


\section{Marktgleichgewichte}

Zum Abschluß der Modellbeschreibung seien nochmals die ein allgemeines Gleichgewicht kennzeichnenden Markträumungsbedingungen tabellarisch aufgeführt.

Faktormarkt: $\quad \sum_{i=1}^{15} K_{i}=\bar{K} \quad$ und $\quad \sum_{i=1}^{15} L_{i}=L$

Gütermarkt: $\quad \sum_{j=1}^{15} a_{i j} Q_{j}+C_{i}+I_{i}+X_{i}-M_{i}=Q_{i}, \quad i=1, \ldots, 14$ und $\quad \sum_{i=1}^{15} a_{15 i} Q_{i}+G=Q_{15}$

Außenhandel:

$$
\sum_{i=1}^{14} q_{i} X_{i}=\sum_{i=1}^{14} q_{i} M_{i}
$$

Die linke Seite der Gleichungen repräsentiert die jeweilige Faktor- bzw. Güternachfrage, die rechte Seite das Angebot. Diese Markträumungsbedingungen müssen - zusammen mit den für den repräsentativen Haushalt und den Staat relevanten Budgetrestriktionen sowie den NullProfit-Bedingungen für die Unternehmen - auch von den im anschlieBenden Kapitel erläuterten Basisdaten erfüllt werden. 
Peter Gottfried - 978-3-631-75208-1

Downloaded from PubFactory at 01/11/2019 07:06:43AM

via free access 


\section{Teil B: Die Erstellung einer konsistenten Datenbasis}

Der vorangehende Teil legte den funktionalen Modellrahmen fest. Die nun folgenden Abschnitte befassen sich mit Problemen, die bei der Erstellung einer mikro-konsistenten Datenbasis auftreten und deren Lösungsversuchen. In gleicher Weise, wie das zugrundeliegende theoretische Modell bestimmte Zusammenhänge nur auf einem sehr hohen Abstraktionsniveau darstellt und andere wiederum sehr detailliert abbildet, muß auch die Datenbasis teils stilisierte, teils stark disaggregierte Sachverhalte numerisch beschreiben. Hierzu zwei kurze Beispiele:

Das theoretische Modell unterstellt Ausgeglichenheit der Leistungsbilanz. Man wird jedoch keine statistische Quelle finden, die diese Implikation des Modells bestätigt, ganz unabhängig davon, nach welcher Abgrenzung der Außenhandel ausgewiesen ist. Die Bundesrepublik Deutschland war im betrachteten Zeitraum (1984) realiter Netto-Exporteur von Waren und Dienstleistungen. Folglich sind die ausgewiesenen Exporte den Importen - oder vice versa - in irgendeiner Form wertmäßig anzupassen, damit das von theoretischer Seite geforderte stilisierte Gleichgewicht entsteht.

Abgesehen von derartigen Modifikationen einzelner Datenelemente einer statistischen Quelle müssen zum Teil auch verschiedene Quellen miteinander kombiniert werden. Es gibt wohl keine Einzelstatistik - zumindest ist dem Autor keine bekannt -, die beispielsweise die Produktionsverflechtung der Unternehmen aufzeigt und die von ihnen zu entrichtenden Körperschaft- oder Gewerbesteuerbeträge als gesonderte Positionen ausweist. Man benötigt daher spezifische Steuerstatistiken, anhand welcher die Positionen der Ausgangsstatistik weiter aufgeschlüsselt und in die gewünschte Darstellungsform gebracht werden können.

Die beiden Beispiele veranschaulichen nicht nur die vorzunehmenden Maßnahmen, sie deuten ebenfalls die grundsätzlichen Schwierigkeiten an, die sofort auftreten, wenn Theorie und empirische Daten oder, wie im zweiten Fall, Daten aus verschiedenen statistischen Quellen miteinander zu verbinden sind. Erstens ist bei den zugrundegelegten Statistiken darauf zu achten, in welcher Darstellungskonzeption die ausgewiesenen Daten vorliegen und ob diese mit der vom theoretischen Modell 
implizit geforderten vereinbar sind. So ist es keineswegs gleichgültig - wie es im ersten Beispiel möglicherweise den Anschein hatte - nach welchem Darstellungskonzept der Außenhandel ausgewiesen wird. Das theoretische Modell beschreibt die Produktion und den Verbrauch von Gütern im Inland. Ausländische Warensendungen auf ein Zollfreilager u. ä. oder inländische Lieferungen von einem Zollfreilager ins Ausland werden dagegen nicht problematisiert. Folglich würden Statistiken, die den Außenhandel nach dem Generalhandelskonzept ausweisen, nicht den theoretisch dargestellten Sachverhalt treffen.

Zweitens ist bei der Verwendung mehrerer Einzelstatistiken zu berücksichtigen, daß die ausgewiesenen Daten in derselben Abgrenzung vorliegen. Beispielsweise sind die Angaben von Volkswirtschaftlichen Gesamtrechnungen traditionellen Stils nicht direkt mit denen von Input-Output-Tabellen vereinbar, obwohl die Darstellungseinheiten in beiden Rechenwerken mitunter den gleichen Namen tragen.

Bevor wir uns jedoch mit der sachspezifischen Problematik näher auseinandersetzen, sei auf einen mehr didaktischen Aspekt hingewiesen, der gerade bei der Erläuterung von Datenmanipulationen sehr deutlich hervortritt: die Frage nach der Ausführlichkeit der Erläuterungen. Einerseits soll der Leser die Notwendigkeit der vorgenommenen Anpassungen erkennen und diese nachvollziehen können. Hält man die Ausführungen auf einem zu allgemeinen Niveau und verzichtet gänzlich auf Einzelheiten, so geht meist die Nachvollziehbarkeit verloren. Es treten gerade aufgrund einer übertriebenen Kürze der Angaben mehr Fragen auf, als tatsächlich beantwortet werden. Andererseits führt die protokollhafte Darlegung aller Zwischenschritte nicht nur zu einer immensen Fülle von mit Zahlen angefülltem Papier, sondern ebenfalls zur raschen Ermüdung und Langeweile beim Lesen dieses für einen theoretisch orientierten Personenkreis vielleicht weniger interessanten Teilbereichs.

Um dem Trade-off zwischen Verständnislosigkeit und Langeweile zu begegnen, wird hier auf ein allzu detailliertes „Entlanghangeln an den Daten“, ebenso wie auf die Angabe von Zwischen ergebnissen verzichtet. Die auftretenden Probleme werden jedoch ausführlich angesprochen und die notwendigen Maßnahmen in einer Form dargestellt, die es dem interessierten Leser ermöglichen sollte, gegebenenfalls selbst die 
einzelnen Zwischenergebnisse zu reproduzieren. Ein Leser, der hingegen nicht an derartigen Problematiken interessiert ist, kann diesen Teil auch gänzlich überspringen - und die Datenbasis als gegeben betrachten -, ohne hierdurch Einbußen an theoretischen Erkenntnissen zu erleiden.

Der nachfolgende Abschnitt erläutert zunächst die wohl wichtigste Datenquelle - die Input-Output-Tabelle -, aus dem Blickwinkel des verwendeten Allgemeinen Gleichgewichtsmodells betrachtet. Im Anschluß daran wird die Angleichung der Basisdaten an die vom theoretischen Modell implizierten Eigenschaften sukzessive, nach Tabellenquadranten geordnet, erklärt.

\section{Die Input-Output-Tabelle des statistischen Bundes- amtes}

Die Mehrzahl der das Ausgangsgleichgewicht beschreibenden Daten stammt direkt oder indirekt aus der vom statistischen Bundesamt für das Jahr 1984 veröffentlichten Input-Output-Tabelle. Aufgrund dieser zentralen Bedeutung der Tabelle, aber auch zum besseren Verständnis der anschließend erläuterten Modifikationen, erscheint es angebracht, einige Bemerkungen über Wesen und Ziel des Rechenwerks den durchgeführten Modifikationen voranzustellen. Dies soll jedoch nicht zu einer umfassenden Diskussion über den Tabellenaufbau sowie der damit verbundenen statistischen Probleme führen - der Leser sei hierzu auf die umfangreiche Literatur z. B. ZWER [1981], HoLUB-SCHNABL [1985] oder STAHMER $[1983,1984]$ zur gegenwärtigen Erstellungspraxis verwiesen -, sondern nur diejenigen konzeptionellen Charakteristika, die einen unmittelbaren Zusammenhang zu dem Gleichgewichtsmodell aufweisen, werden angesprochen. Damit wird versucht, die wechselseitigen Beziehungen zwischen der Vorgehensweise des statistischen Bundesamtes auf der einen und den modelltheoretischen Implikationen auf der anderen Seite offenzulegen.

Die vom statistischen Bundesamt verwendete Darstellungskonzeption kommt den modelltheoretischen Erfordernissen prinzipiell sehr entgegen. Auf der Basis von fachlichen Unternehmensteilen werden 58 homogene Produktionsbereiche gebildet, welche sich durch eine relativ einheitliche Produktionstechnologie, d. h. Inputstruktur und durch eine 
einheitliche Verwendung der produzierten Güter auszeichnen. Für diese fiktiven Darstellungseinheiten sind in der Tabelle Produktions- und Verbrauchswerte ausgewiesen, die insbesondere auch unternehmensinterne Vorleistungsströme beinhalten. So zeigt bspw. die $j$-te Spalte der Zentralmatrix, was an Leistungen aus den Bereichen $1, \ldots, n$ wertmäßig verbraucht wurde, um das Produktionsvolumen von Gut $j$ zu erhalten.

Alle inländischen Güterströme sind einheitlich mit Ab-Werk-Preisen - Anschaffungspreise (ohne Umsatzsteuer) vermindert um Handelsund Transportspanne - bewertet, für die Importe werden entsprechend Ab-Zoll-Preise - Anschaffungspreise (ohne Einfuhrumsatzsteuer) vermindert um Handels- und Transportspanne ab Zollgrenze - herangezogen $^{88}$. Zweck der Verwendung dieses Preiskonzepts ist ebenfalls die Betonung der technologischen Verflechtung, die primär durch die Mengenkomponente der ausgewiesenen Wertgrößen zum Ausdruck kommt. Verbrauchen bspw. zwei durch unterschiedliche Standorte gekennzeichnete Unternehmen die gleiche Menge eines nicht selbst produzierten Gutes, würde eine Bewertung der Mengen zu Anschaffungspreisen, bedingt durch verschieden hohe Transportkosten, zu unterschiedlichen Wertangaben führen. Ähnliches gilt, falls einem der beiden Unternehmen wegen seiner Marktposition besondere Konditionen, z. B. ein „Mengenrabatt“, eingeräumt werden. Hier wäre die Proportionalität zwischen Mengen- und Wertgröße aufgrund unterschiedlicher Handelsspannen zerstört.

Aus theoretischer Sicht bedürfen die Vorzüge dieses funktional orientierten Darstellungskonzepts kaum einer näheren Erläuterung. Bei der Aufbereitung des Datenmaterials berücksichtigt das Statistische Bundesamt offensichtlich genau die Vorstellungen, die auch der theoretischen Beschreibung der Produktionsverflechtung unterliegen. Ferner harmoniert die Bewertung aller Güter zu Ab-Werk-Preisen mit der theoretischen Implikation, daß bei vollkommener Konkurrenz nur ein einziger Gleichgewichtspreis pro Gut oder Faktor existiert.

War bislang von der Input-Output-Tabelle (mit 58 Produktionsbe-

${ }^{88}$ Als Anschaffungspreis wird hierbei derjenige Preis bezeichnet, den der Abnehmer der Ware zu entrichten hat ( $\approx$ Marktpreis). Nähere Erläuterungen siehe STBA [1988], S. 18f. 
reichen) die Rede, mag das etwas irritiert haben, denn das Statistische Bundesamt publiziert zwei Tabellen dieser Aggregationstiefe. Sie unterscheiden sich in der Behandlung der Importe. In der ersten Version sind Importe zusammen mit den entsprechenden inländischen Erzeugnissen in den Felderwerten der Zentral- und der Endnachfragematrix ausgewiesen, so daß eine Unterscheidung zwischen inländischem und importiertem Gut nicht möglich ist. Diє Zeilensumme über beide Quadranten hinweg ergibt damit das gesamtwirtschaftliche Aufkommen an inländisch erzeugten und importierten Gütern, während spaltenweise vornehmlich die Verbrauchswerte der inländischen Produktion zum Ausdruck kommen. Der Ausgleich von Zeilen- und Spaltensummen erfolgt innerhalb des Wertschöpfungsquadranten über die nach Güterarten geordnete Korrekturzeile gleichartige Importe.

In der zweiten Version beziehen sich die Angaben der Zentral- und der Endnachfragematrix nur auf inländisch produzierte Güter. Dementsprechend gibt die Zeilensumme hier nur das inländisch erzeugte Gütervolumen an. Die Importe sind unabhängig von der Güterart unterhalb der Zentral- und der Endnachfragematrix in einer Zeile Vorleistung der Produktionsbereiche bzw. letzte Verwendung von Gütern aus Einfuhr nach den Produktions- bzw. Verwendungsbereichen zusammengefaßt, in denen sie tatsächlich verbraucht wurden.

Die Beschreibung der zwei Ausweisarten ist sicherlich nicht umfassend $^{89}$. Sie genügt jedoch, um auf die jeweils spezifischen Nachteile der Darstellungen aufmerksam zu machen: Während nach der ersten Verbuchungsart die Verwendung der Importe nicht erkennbar ist, kann aus der zweiten nicht das Importvolumen pro Güterart entnommen werden. Erinnern wir uns an die theoretische Annahme, daß die Importe vollkommene Substitute zu den entsprechenden heimischen Gütern darstellen. Von der Verwendungsseite betrachtet, ist es somit irrelevant, ob die Güter importiert oder im Inland produziert werden. Nur die insgesamt je Güterart nachgefragten Mengen sind von Bedeutung. Für die numerische Beschreibung des Ausgangsgleichgewichts ist folglich die zuerst skizzierte Ausweistechnik relevant.

Damit sind die der Tabelle unterliegenden wichtigsten Basiskonzepte

${ }^{89}$ Eine detaillierte schematische Darstellung aller Ausweisarten befindet sich bspw. in HolUB-SCHNABL [1985], S. $53 \mathrm{ff}$. 
zumindest etwas verdeutlicht worden. Ebenso dürfte nun auch erkennbar sein, warum die Tabelle des Statistischen Bundesamtes als Datengrundlage besser geeignet erscheint, als bspw. die vom DIW erstellte, die eher auf die Darstellung von Markttransaktionen abzielt und nicht auf produktionstechnische Zusammenhänge. Nachfolgend werden nun die drei Tabellenquadranten - Matrix der Produktionsverflechtung, Matrix der Primärinputs und Endnachfragematrix - im einzelnen betrachtet, wobei auf den Aussagegehalt ihrer einzelnen Positionen nur dann eingegangen wird, wenn er von den modellmäßig erforderlichen abweicht.

Aus den oben genannten Gründen können die Angaben der Produktionsverflechtungsmatrix - in geeigneter Zusammenfassung - nahezu direkt übernommen werden. Der nächste Abschnitt konzentriert sich deshalb auf die hinsichtlich der Umsatzsteuerregelungen günstigste Aggregation der 58 Produktionsbereiche und die Ermittlung von (nominalen) durchschnittlichen Umsatzsteuersätzen.

\section{Zentralmatrix und nominelle Umsatzsteuersätze}

Seit 1978 erfolgt der Ausweis der Input-Output-Tabellen nach dem sog. Nettosystem, d. h. die in Produktionsverflechtungs- und Endnachfragematrix ausgewiesenen Wertströme sind exklusive Umsatzsteuer verbucht. Diese wird in einer gesonderten Zeile nichtabzugsfähige Umsatzsteuer unterhalb der beiden Quadranten denjenigen Verwendungskategorien zugeordnet, die sie tatsächlich tragen. Nichtabzugsfähige Umsatzsteuer ist dann auszuweisen, wenn der Empfänger einer Leistung den auf ihren Bezug anfallenden Umsatzsteuerbetrag nicht als Vorsteuer geltend machen kann. Hauptsächlich sind hiervon die Konsumgüterspalte, zum Teil aber auch der Vorleistungsverbrauch und die Investitionen betroffen.

Ein Übergang von Produktionsbereichen auf modellgerechte Sektoren bereitet deshalb Schwierigkeiten, da jeder Sektor annahmegemäß nur ein homogenes Gut herstellt, auf dessen Umsatz konsequenterweise nur ein Steuersatz angewendet werden $k_{a n n}{ }^{90}$. Zwar sind die in einem Produktionsbereich ausgewiesenen Gütergruppen homogen hinsichtlich

${ }^{90}$ Um Verwechslungen zwischen statistischen Darstellungseinheiten und modell- 
der Erzeugung und Verwendung, die auf die einzelnen Güter eines Produktionsbereichs anzuwendenden Umsatzsteuersätze können jedoch differieren. Zum Beispiel ist für Produktionsbereich 1: Erzeugung von Produkten der Landwirtschaft aufgrund seiner Güterstruktur gleich eine ganze Palette unterschiedlicher Steuersätze - nämlich 5,7,8 und 13 Prozent; geregelt in $\S 12$ UStG, der Anlage zu $\S 12$ Abs. 2 Nr. 1 UStG und $\S 24$ UStG - relevant. Würde man nun Produktionsbereich 1 als Sektor 1 abbilden, stellt sich unmittelbar die Frage, mit welchem Steuersatz „das Gut" dieses Sektors zu besteuern wäre. Die hier gewählte Antwort auf diese Frage wurde bereits in Kapitel II, Abschnitt (5) angedeutet: Man repräsentiert die verschiedenen tatsächlichen durch einen gemittelten Steuersatz. Wie dieser nominale Durchschnittsteuersatz bestimmt werden kann, ist noch zu erläutern. Sein genauer Wert hängt offensichtlich davon ab, welche Produktionsbereiche man in einem Sektor zusammenfaßt.

Die Bildung derartiger Durchschnittsteuersätze kann für jeden beliebigen Sektor vorgenommen werden, mit Ausnahme derjenigen, die ein steuerbefreites Gut herstellen. Für sie muß der Umsatzsteuersatz gleich Null sein, was zu einem weiteren Problem führt. Die Tabelle enthält 3 Produktionsbereiche, die sowohl steuerbefreite als auch besteuerte Gütergruppen in signifikanten Größenordnungen enthalten. Im einzelnen sind das Produktionsbereich 53: Leistungen der Wissenschaft und Kultur und der Verlage, er enthält steuerbefreite wissenschaftliche und kulturelle Dienstleistungen ( $\$ 4$ UStG) und zum ermäßigten Steuersatz (7 v. H.) pflichtige Verlagserzeugnisse (Anlage zu $\S 12$ Abs. 2 Nr. 1 UStG); Produktionsbereich 54: marktbestimmte Dienstleistungen des Gesundheits- und Veterinärwesens, der steuerbefreite medizinische (§4 UStG), zum ermäßigten Satz besteuerte zahntechnische (Anlage zu $§ 12$ Abs. 2 Nr. 1 UStG) und dem allgemeinen Steuersatz (14 v. H.) unterliegende tierärztliche Leistungen umfaßt und schließlich Produktionsbereich 55: sonstige marktbestimmte Dienstleistungen, der beispielsweise die steuerbefreiten Dienstleistungen von Versicherungsmaklern und Rechtsanwälten ( $\$ 4$ UStG), aber auch die zum normalen Satz

theoretischen Wirtschaftseinheiten vorzubeugen, sei an dieser Stelle eine kurze Begriffsabgrenzung eingefügt. Die der Input-Output-Tabelle unterliegende statistische Darstellungseinheit wird durchgehend als Produktionsbereich, das idealtypische Unternehmen als Produktionssektor oder kurz Sektor bezeichnet. 
besteuerten Leistungen der Friseure enthält - eine detailliertere Auflistung aller Dienstleistungen und deren Besteuerung erscheint relativ müßig. Da aufgrund der gesetzlichen Koppelung von Besteuerung mit Abzugsberechtigung der Vorsteuer bzw. von Steuerbefreiung mit Ausschluß des Vorsteuerabzugsrechts nur die zwei Möglichkeiten bestehen, entweder für den Sektor einen Durchschnittsteuersatz anzunehmen, wobei die von ihm geleistete Vorsteuer gänzlich seine BasisUmsatzsteuerschuld vermindert, oder den Sektor von der Umsatzsteuer zu befreien und nur dann nichtabzugsfähige Umsatzsteuer auf die von ihm eingesetzten Zwischenprodukte anfallen $\mathrm{kann}^{91}$, müssen die oben genannten Produktionsbereiche zunächst disaggregiert werden, um besteuerte und steuerbefreite Gütergruppen zu trennen.

Diese beiden Eigenschaften, daß einerseits die Höhe der durchschnittlichen Umsatzsteuersätze von der Sektorbildung abhängt, und daß andererseits jeder Sektor entweder ein besteuertes oder ein steuerbefreites homogenes Gut herstellen kann, bestimmen die weitere Vorgehensweise. In einem ersten Schritt werden zunächst näherungsweise 15 Produktionssektoren gebildet, wobei insbesondere "Sektor“ $12^{*}$ nicht die geforderte Homogenität hinsichtlich der Besteuerung aufweist, da er die Produktionsbereiche 53-55 umfaßt. Auf der Basis dieser Sektoreinteilung werden im zweiten Schritt die durchschnittlichen Umsatzsteuersätze ermittelt. Mit Hilfe dieser zusätzlichen Information kann anschließend der inhomogene Sektor $12^{*}$ in zwei homogene Teilsektoren aufgeschlüsselt werden. Der besteuerte Teil bildet nun für sich genommen den endgültigen Sektor 12, der steuerbefreite Teil wird dem

91 Streng genommen ist diese Darstellung nicht ganz vollständig, da auch eine "versteckte" nichtabzugsfähige Umsatzsteuer oder Subvention auftreten kann. Bedingt durch die gesetzlich geregelte Anwendung von Durchschnittsätzen auf die Umsätze und die Vorleistungen von landwirtschaftlichen Betrieben ( $\$ 24$ UStG ggf. in Verbindung mit Kürzungen der Bemessungsgrundlage § 24a UStG) sowie sonstiger Unternehmen ohne besondere Aufzeichnungspflicht ( $\$ 23$ UStG) ist nicht gewährleistet, daß diesen Unternehmen keine steuerlichen Vor- oder Nachteile entstehen. Zwar sind die Durchschnittsätze so festgelegt, daß sie „... zu einer Steuer führen, die nicht wesentlich von dem Betrage abweicht, der sich nach diesem Gesetz ohne Anwendung der Durchschnittsätze ergeben würde" (§ 23 Abs. 2 UStG), dennoch ist im Einzelfall eine überhöhte oder zu niedrige Steuerzahllast nicht auszuschließen. Wir wollen jedoch davon ausgehen, daß die gesetzlich angestrebte Kongruenz erfüllt ist. 
bislang ebenfalls angenäherten Sektor $14^{*}$ zugeordnet. Als letzter Arbeitsgang ist schließlich noch der Durchschnittsteuersatz für Sektor 12* der endgültigen Sektoreinteilung anzupassen, die restlichen Steuersätze bleiben davon unberührt.

Die Schritte im einzelnen: Die Aggregation der 58 Produktionsbereiche auf 15 Sektoren erfolgt in Anlehnung an die "kleine“, in 12 Produktionsbereiche zusammengefaßte, Input-Output-Tabelle des Statistischen Bundesamtes, wobei die Sektoren $1-12^{*}$ annahmegemäß besteuerte, die Sektoren $13,14^{*}$ und 15 steuerbefreite Güter herstellen. Aus welchen Produktionsbereichen sie sich im einzelnen zusammensetzen, kann - mit Ausnahme der Sektoren $12^{*}$ und $14^{*}$ - aus den ersten beiden Spalten der Tabelle 7 entnommen werden. Die Tabelle zeigt bereits die endgültige Struktur der Produktionssektoren an, in der Ausgangssituation besteht Sektor $14^{*}$ aus den Produktionsbereichen 49-51 und Sektor $12^{*}$, wie erwähnt, aus 53-55.

Für die Ermittlung der Durchschnittsteuersätze kann folgende Bedingung genutzt werden. Ordnet man jedem Sektor einen durchschnittlichen Umsatzsteuersatz zu und berechnet die Vorsteuerbeträge auf den Inputverbrauch eines steuerbefreiten Sektors, ergibt sich mit der Summe dieser Beträge die für den Sektor relevante nichtabzugsfähige Umsatzsteuer. Sie kann in entsprechender Aggregation unmittelbar der Zentralmatrix entnommen werden. Da die Zusammenfassung der Produktionsbereiche so vorgenommen wurde, daß die letzten drei Sektoren einheitlich von der Umsatzsteuer befreit sind, müssen im Unternehmensbereich die drei Gleichungen

$$
N U_{i}^{u}=\sum_{j=1}^{15} \tau_{j}^{u} q_{j} A_{j i}, \quad i=13,14^{*}, 15
$$

erfüllt sein. Ebenso muß die Summe der Umsatzsteuerbeträge auf die Netto-Ausgaben für Konsumgüter $q_{i} C_{i}$ der in der Endnachfragematrix angegebenen nichtabzugsfähigen Umsatzsteuer $N U^{c}$ mit

$$
N U^{c}=\sum_{j=1}^{15} \tau_{j}^{u} q_{j} C_{j}
$$

entsprechen. Die unterhalb der Investitionsgüterspalte ausgewiesene 
Umsatzsteuer kann nicht als weitere Bedingung herangezogen werden, da der Spalte lediglich die Verwendung, nicht aber der Verwendungsort bzw. der Investitionsgüter verbrauchende Sektor zu entnehmen ist. Hierzu wäre eine komplette Investitionsverflechtungsmatrix erforderlich.

Zur Ermittlung der nominellen Durchschnittsteuersätze stehen somit insgesamt vier Gleichungen zur Verfügung, mit denen vier Steuersätze endogen berechenbar sind. Aufgrund der Steuerbefreiung der letzten drei Sektoren ist $\tau_{13}^{u}, \ldots, \tau_{15}^{u}=0$ unmittelbar vorgegeben. Die verbleibenden acht Sätze sind so vorzugeben, daß die endogen berechneten „realistische“ Werte annehmen; mit anderen Worten, sie müssen in einem Wertebereich zwischen 0.07 und 0.14 liegen.

Diese Wahl wird durch den Umstand erleichtert, daß die Umsatzsteuerregelungen hauptsächlich gütermäßig orientiert sind und nicht institutionell. Anhaltspunkte für die vorzugebenden Steuersätze, aber auch zur Kontrolle der endogen berechneten, liefert in erster Linie das Umsatzsteuergesetz - hier vor allem $\S 4$ Steuerbefreiungen bei Lieferungen, sonstigen Leistungen und Eigenverbrauch; 112 Steuersätze in Verbindung mit der Anlage zu $\S 12$ Liste der dem ermäBigten Steuersatz unterliegenden Gegenstände; $§ 24$ Durchschnittsätze für land- und forstwirtschaftliche Betriebe zusammen mit $\S 24 a$ Kürzungsansprüche für land- und forstwirtschaftliche Umsätze - und ein Vergleich dieser gesetzlichen Regelungen mit der Güterstruktur der Produktionsbereiche (STBA [1988b], S. 184 ff.), bzw. der ihr unterliegenden Systematik der Wirtschaftszweige (STBA [1982]). Weitere Anhaltspunkte können der Umsatzsteuerstatistik durch Vergleich von steuerbarem Umsatz und der Umsatzsteuer vor Abzug der Vorsteuerbeträge ${ }^{92}$ in relativ starker Disaggregation nach Wirtschaftszweigen entnommen werden.

In beiden Fällen handelt es sich jedoch nur um Anhaltspunkte, was bereits an Produktionsbereich 1 - nun enthalten in Sektor 1 - erkennbar ist. Man weiß zwar, daß für die Umsätze dieses Produktionsbereichs eine ganze Reihe unterschiedlicher Steuersätze zur Anwendung kommen, in welchen Relationen die Umsätze zueinander stehen, ist jedoch schwer abzuschätzen. Ebenso geht beispielsweise aus der Systematik der Wirtschaftszweige hervor, daß zu Produktionsbereich 9 Herstel-

${ }^{92} \mathrm{Zu}$ finden in STBA [1986a], S. $39 \mathrm{ff}$. 
lung von chemischen Erzeugnissen, Spalt- und Brutstoffen u. a. auch Fette bzw. Öle (WZ 20010), Aromastoffe (WZ 20024), Pektinstoffe (WZ 20027 ) usw. zählen, welche dem ermäßigten Steuersatz unterliegen (Anlage zu $\S 12$ Ziffer 21,40,19), aber wiederum ist das Verhältnis der zum allgemeinen Steuersatz und der zum ermäßigten Satz besteuerten Umsätze nicht abzusehen. Eine ähnliche Situation ist in nahezu allen Produktionsbereichen vorzufinden.

Auch die zweite Datenquelle - die Umsatzsteuerstatistik - erlaubt keine unmittelbare Berechnung der Durchschnittsteuersätze. Zwar sind Umsätze und Umsatzsteuerbeträge direkt ablesbar, doch erfolgt die Zuordnung der statistischen Einheiten hier nach dem Schwerpunktprinzip und nicht, wie in der Input-Output-Tabelle, funktional orientiert, so daß beiden Statistiken unterschiedliche Aggregate zugrundeliegen. Anhand der beiden Orientierungshilfen kann somit bestenfalls festgestellt werden, daß der für einen Produktionssektor relevante Durchschnittsteuersatz innerhalb einer bestimmten Bandbreite liegen muß. Es besteht nur die Möglichkeit, für acht (beliebig ausgewählte) Sektoren Durchschnittsätze innerhalb dieser Bandbreiten anzunehmen und anhand der daraus resultierenden endogen berechneten - im konkreten Fall wurde das Gleichungssystem nach den Steuersätzen der Sektoren 1,3,9 und 10 aufgelöst - Korrekturen vorzunehmen. Die angenommenen Steuersätze müssen dann so lange nach oben oder nach unten korrigiert werden, bis auch die errechneten Sätze zulässige Werte annehmen. Entscheidungshilfe bei der Auswahl und der Veränderungsrichtung des zu korrigierenden Steuersatzes bietet neben den oben genannten Orientierungspunkten die Inputstruktur der steuerbefreiten Sektoren. In der letzten Spalte von Tabelle 7 sind die auf diese Weise ermittelten Durchschnittsteuersätze aufgelistet.

Diese Vorgehensweise mag etwas pragmatisch erscheinen, andererseits reagieren die endogenen Werte sehr sensitiv auf nur geringfügige Änderungen der Vorgaben und sind schnell außerhalb der zulässigen Spanne. Reduziert man beispielsweise den für Sektor 5 maßgeblichen Steuersatz von 14 auf 13\%, ergäbe sich für Sektor 1 ein endogener Satz von $-6.4 \%$, für Sektor 10 ein Satz von $16 \%$. Trotz dieser Sensitivität ist dennoch nicht auszuschließen, daß neben der ermittelten eine Reihe 
Tabelle 7: Sektoreinteilung und nominale Durchschnittsteuersätze

\begin{tabular}{|c|c|c|c|}
\hline Nr. & Sektorbezeichnung & $\begin{array}{l}\text { Produktionsbereiche } \\
\text { nach STBA. }\end{array}$ & $\begin{array}{c}\text { nominaler } \\
\text { Durchschnitt- } \\
\text { steuersatz (in \%) }\end{array}$ \\
\hline 1 & Land- und Forstwirtschaft & $1-2$ & 7.2 \\
\hline 2 & $\begin{array}{l}\text { Energie, Wasser und } \\
\text { Bergbau }\end{array}$ & $3-8$ & 13.1 \\
\hline 3 & Chemische Erzeugnisse & $9-12$ & 12.3 \\
\hline 4 & $\begin{array}{l}\text { Metallerzeugnisse, Maschi- } \\
\text { nenbau }\end{array}$ & $13-22$ & 14 \\
\hline 5 & Fahrzeuge & $23-25$ & 14 \\
\hline 6 & $\begin{array}{l}\text { E-technische und feinme- } \\
\text { chanische Erzeugnisse }\end{array}$ & $26-29$ & 13.6 \\
\hline 7 & $\begin{array}{l}\text { Holz- und Papierverar- } \\
\text { beitung }\end{array}$ & $30-34$ & 12.5 \\
\hline 8 & Leder und Bekleidung & $35-37$ & 14 \\
\hline 9 & $\begin{array}{l}\text { Nahrungsmittel, Getränke } \\
\text { und Tabak }\end{array}$ & $38-40$ & 7.5 \\
\hline 10 & $\mathrm{Bau}$ & $41-42$ & 14 \\
\hline 11 & Handel und Transport & $43-46,48,52$ & 12.9 \\
\hline 12 & $\begin{array}{l}\text { sonst. marktbestimmte } \\
\text { Dienstleistungen }\end{array}$ & T.v. 53-55 & 10.4 \\
\hline 13 & $\begin{array}{l}\text { Post und priv. Organisa- } \\
\text { tionen ohne Erwerbszweck }\end{array}$ & 47,58 & 0 \\
\hline 14 & $\begin{array}{l}\text { Banken, Versicherungen, } \\
\text { Wohnungsvermietung usw. }\end{array}$ & $\begin{array}{l}49-51 \\
\text { T.v. } 53-55\end{array}$ & 0 \\
\hline 15 & Staat & $56-57$ & 0 \\
\hline
\end{tabular}

weiterer Steuersatzstrukturen existieren ${ }^{93}$, die ebenfalls die an sie gestellten Anforderungen erfüllen. Ziel der Arbeitsstufe ist auch nicht die Berechnung von den „tatsächlichen“ Durchschnittsteuersätzen per se, sondern das Auffinden einer modellkonformen (und einigermaßen

${ }^{93} \mathrm{Zu}$ denken ist hier in erster Linie an den Genauigkeitsgrad der gemittelten Sätze im Nachkommastellenbereich. 
realistischen) Darstellungsweise, welche die tatsächlich ausgewiesenen Umsatzsteuerbeträge erzeugt.

Anhand der nominalen Durchschnittsteuersätze ist es nun möglich Sektor $12^{*}$ in zwei homogene Teile aufzuspalten. Wurde zuvor für die Steuersatzberechnung (stillschweigend) angenommen, daß der Sektor ein einziges steuerpflichtiges Gut produziert und damit zum Abzug der Vorsteuer berechtigt ist, unterstellt man nun, daß der Sektor steuerbefreit wäre und errechnet die auf seinen Vorleistungsverbrauch anfallende nichtabzugsfähige Umsatzsteuer. Dieser Betrag ist wesentlich höher als der in der Input-Output-Tabelle tatsächlich ausgewiesene Wert, da sich letzterer sowohl auf das befreite als auch auf das besteuerte Gut des Sektors $12^{*}$ bezieht. Nimmt man an, beide Güter unterscheiden sich nicht oder nur geringfügig hinsichtlich ihrer Produktionsstruktur, dann gibt die Relation von ausgewiesener zu berechneter nichtabzugsfähiger Umsatzsteuer an, wieviel Prozent eines jeden Inputs zur Erzeugung des steuerbefreiten Gutes benötigt wurden. Man multipliziert folglich alle Inputwerte (einschließlich der Wertschöpfung) des Sektors mit dieser Verhältniszahl und erhält den zur Produktion des steuerbefreiten Gutes relevanten Inputeinsatz. Die Differenz zwischen der ursprünglichen und der neuen Spalte ergibt konsequenterweise die Verbrauchswerte des besteuerten Gutes.

Die gleiche Methode wurde (in Ermangelung besser geeigneter Alternativen) auch für die Aufspaltung der Verwendungsrichtung gewählt. Figur 11 zeigt schematisch die Umbuchung der betreffenden Teilspalte bzw. -zeile auf. Während das einem nominalen Durchschnittsteuersatz unterliegende Gut für sich genommen nun Sektor 12 bildet, ergibt der steuerbefreite Teil zusammen mit Sektor $14^{*}$ das von Sektor 14 letztendlich produzierte homogene Gut.

Als letzter Schritt ist noch der Durchschnittsteuersatz für Sektor 12 neu zu berechnen. Da die Aufspaltung des Sektors sowohl in Verbrauchs- als auch in Verwendungsrichtung anhand eines einheitlichen Faktors vollzogen wurde, genügt es, eine beliebige aus den vier zur Verfügung stehenden Gleichungen heranzuziehen und nach Sektor 12 aufzulösen. Die Durchschnittsteuersätze $\tau_{1}^{u}, \ldots, \tau_{11}^{u}$ und $\tau_{13}^{u}, \tau_{14}^{u}$ und $\tau_{15}^{u}$ bleiben unverändert. Insbesondere produziert Sektor 14 - wie zuvor auch der angenäherte Sektor $14^{*}$ - ein steuerbefreites Gut. 
Figur 11: Skizzierte Umbuchung von Sektor $12^{*}$

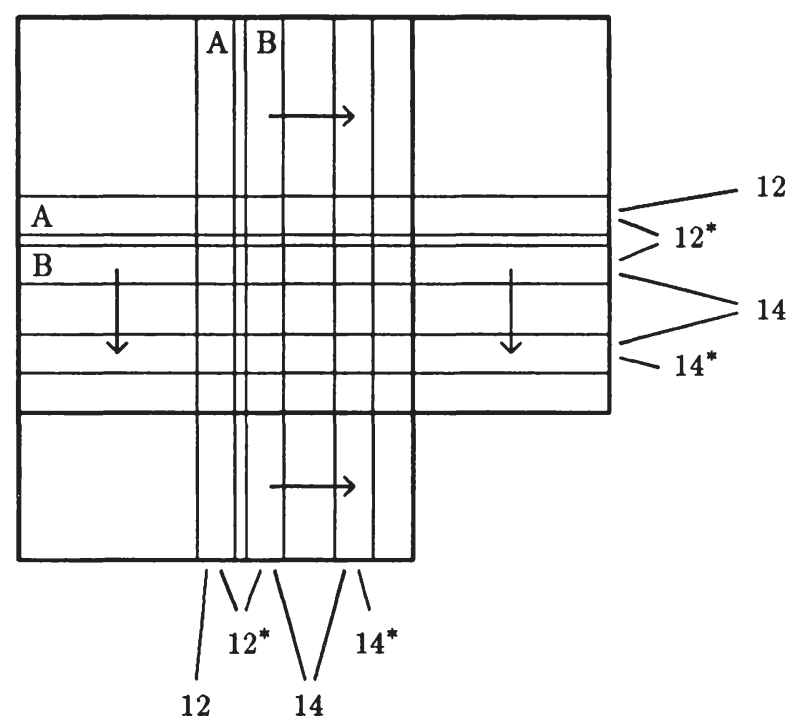

A: Steuerpflichtiges Gut von $12^{*}$

B: Steuerbefreites Gut von $12^{*}$

Mit dieser Sektoreinteilung stehen die die Produktionsverflechtung beschreibenden Daten größtenteils fest. Sie entstammen - entsprechend aggregiert - unmittelbar der Zentralmatrix der Input-Output-Tabelle und können aus Tabelle 8 entnommen werden.

Eine Ausnahme bildet jedoch die nun zu erläuternde modifizierte Behandlung des Produktionsbereichs 49 Leistungen der Kreditinstitute. Auf statistischer Ebene unterscheidet man zwei Arten von Erlösen aus Dienstleistungen des Produktionsbereichs 49:

(1) Erlöse aus Dienstleistungen gegen Zahlung eines Marktpreises, wie zum Beispiel die Vermietung von BankschlieBfächern oder Maklergebühren, und 
(2) Erlöse aus unterstellter Produktion von Bankdienstleistungen, die im wesentlichen aus der Differenz zwischen Soll- und Haben-Zinsen entstehen.

Während der Verbrauch von (1) in der Input-Output-Tabelle direkt den Produktionsbereichen zurechenbar ist, kann (2) nur sehr schwer auf die verbrauchenden Bereiche verteilt werden. Aus diesem Grund wird die unterstellte Produktion von Bankdienstleistungen von statistischer Seite als Eigenverbrauch verbucht. Sie vermindert damit - abgesehen von dem Anteil, der tatsächlich intern verbraucht wird - die Nettowertschöpfung (zu Faktorkosten) des Produktionsbereichs 49 in vollem Umfang. Gleichzeitig erhöht sich auch die Wertschöpfung der restlichen Produktionsbereiche um genau den Betrag, um den sie die unterstellte Produktion von Bankdienstleistungen in Anspruch nehmen ${ }^{94}$. Gerade diese Konsequenzen sind unserer Zielsetzung jedoch eher gegenläufig, zumal Banken und Kreditinstitute zu den Unternehmen zählen, die von der Umsatzsteuer befreit und zum Abzug der Vorsteuer nicht berechtigt sind. Deshalb wurden die unterstellten Dienstleistungen in Höhe von 84070 Mill. $\mathrm{DM}^{95}$ aus dem Eigenverbrauch herausgenommen und zeilenweise proportional zu den direkten Dienstleistungen verteilt.

Damit ist der Datenteil, der den intermediären Inputeinsatz im Ausgangsgleichgewicht quantifiziert, erzeugt. Die zum Ausgleich von Zeilen- und Spaltensummen notwendige Gegenbuchung der unterstellten Bankdienstleistungen erfolgt im Wertschöpfungsquadranten und wird entsprechend dort erläutert.

94 Nähere Erläuterungen siehe ZWER [1981], S. $127 \mathrm{ff}$.

${ }^{95}$ Aus STBA [1988a], S. 268. 
Tabelle 8: Zentralmatrix (in 100000000 DM)

\begin{tabular}{|c|rrrrrrrr}
\hline$A_{j i}$ & \multicolumn{1}{c}{1} & \multicolumn{1}{c}{2} & \multicolumn{1}{c}{3} & \multicolumn{1}{c}{4} & \multicolumn{1}{c}{5} & 6 & 7 & 8 \\
\hline 1 & 91.46 & 0.46 & 7.29 & 0.89 & 0.28 & 0.62 & 28.23 & 21.53 \\
2 & 21.53 & 508.28 & 547.25 & 249.91 & 21.04 & 19.30 & 30.07 & 12.40 \\
3 & 96.63 & 42.51 & 1041.75 & 142.48 & 122.29 & 130.50 & 86.48 & 57.60 \\
4 & 19.63 & 63.98 & 46.29 & 1544.78 & 238.49 & 253.10 & 20.14 & 4.66 \\
5 & 5.07 & 2.60 & 1.92 & 11.67 & 325.97 & 2.90 & 4.70 & 1.90 \\
6 & 4.40 & 19.77 & 32.22 & 155.37 & 112.01 & 207.35 & 29.20 & 7.03 \\
7 & 6.10 & 3.04 & 36.33 & 34.62 & 12.28 & 31.23 & 286.66 & 5.51 \\
8 & 0.68 & 0.75 & 7.33 & 1.88 & 14.17 & 3.15 & 12.79 & 208.38 \\
9 & 99.84 & 0.62 & 8.99 & 1.00 & 1.19 & 1.14 & 0.33 & 0.21 \\
10 & 6.23 & 32.09 & 4.09 & 11.11 & 4.71 & 2.60 & 3.06 & 1.53 \\
11 & 48.84 & 32.87 & 172.09 & 255.20 & 127.68 & 115.72 & 89.64 & 46.40 \\
12 & 14.82 & 31.59 & 113.67 & 93.10 & 77.31 & 83.50 & 41.49 & 29.78 \\
13 & 1.38 & 3.80 & 16.88 & 19.69 & 6.34 & 10.50 & 6.70 & 5.09 \\
14 & 11.67 & 25.86 & 57.15 & 99.14 & 38.18 & 51.29 & 25.86 & 19.28 \\
15 & 3.22 & 7.03 & 20.81 & 22.02 & 9.86 & 7.27 & 3.93 & 2.91 \\
\hline$\sum$ & 431.50 & 775.25 & 2114.06 & 2512.86 & 1111.80 & 920.17 & 669.28 & 424.21 \\
\hline$N U_{i}^{u}$ & 0.00 & 0.00 & 0.00 & 0.00 & 0.00 & 0.00 & 0.00 & 0.00 \\
\hline
\end{tabular}




\begin{tabular}{rrrrrrr|r|}
\hline \multicolumn{1}{r}{9} & \multicolumn{1}{c}{10} & \multicolumn{1}{c}{11} & \multicolumn{1}{c}{12} & \multicolumn{1}{c}{13} & \multicolumn{1}{c}{14} & \multicolumn{1}{c}{15} & $\sum$ \\
\hline 594.39 & 0.22 & 40.82 & 12.64 & 5.25 & 26.30 & 15.01 & 845.39 \\
32.08 & 2.38 & 91.86 & 13.76 & 9.75 & 49.32 & 62.47 & 1671.40 \\
53.28 & 57.18 & 183.23 & 79.16 & 21.63 & 38.02 & 170.00 & 2322.74 \\
24.30 & 201.94 & 40.45 & 13.60 & 3.04 & 8.63 & 27.39 & 2510.42 \\
6.15 & 1.45 & 43.95 & 4.59 & 2.77 & 3.36 & 113.77 & 532.77 \\
24.39 & 38.16 & 25.06 & 57.85 & 6.12 & 36.88 & 93.57 & 849.38 \\
36.37 & 37.48 & 86.89 & 132.06 & 5.25 & 67.93 & 35.32 & 817.43 \\
1.04 & 1.64 & 8.67 & 9.59 & 3.61 & 5.22 & 11.77 & 290.67 \\
434.07 & 0.20 & 166.03 & 25.92 & 27.87 & 11.12 & 30.95 & 809.48 \\
3.07 & 16.06 & 20.18 & 9.40 & 4.15 & 150.78 & 63.38 & 332.44 \\
153.99 & 68.23 & 457.83 & 74.95 & 23.24 & 59.65 & 156.70 & 1753.03 \\
57.22 & 51.38 & 220.98 & 218.58 & 15.17 & 214.66 & 257.56 & 1520.81 \\
5.58 & 6.92 & 59.00 & 27.56 & 22.74 & 38.70 & 305.74 & 536.63 \\
31.60 & 32.63 & 407.21 & 162.31 & 35.13 & 377.62 & 370.03 & 1744.96 \\
11.56 & 4.75 & 23.25 & 14.26 & 2.98 & 92.34 & 224.05 & 450.24 \\
\hline 1469.09 & 520.98 & 1875.41 & 856.23 & 188.70 & 1180.53 & 1937.71 & 16987.78 \\
\hline 0.00 & 0.00 & 0.00 & 0.00 & 14.37 & 80.91 & 126.92 & 222.20 \\
\hline
\end{tabular}




\section{Die Matrix der Primärinputs}

Neben Daten, welche die Produktionsverflechtung der Sektoren im Gleichgewicht beschreiben, werden Angaben für den Einsatz von Arbeit und Kapital, für die Abschreibungen sowie für die Produktion- und Kapitalsteuern benötigt. Die Primärinputmatrix der Input-OutputTabelle liefert (in der im letzten Abschnitt entwickelten sektoralen Gliederung) Angaben zu folgenden Positionen:

- Abschreibungen

- Produktionssteuern abzüglich Subventionen

- Einkommen aus unselbständiger Arbeit

- Einkommen aus Unternehmertätigkeit und Vermögen

Steuern, die auf den Einsatz von Kapital gerichtet sind, werden nicht gesondert ausgewiesen. Aber auch die Wertangaben der aufgeführten Positionen sind, wiederum bedingt durch spezifische Eigenschaften des theoretischen Modells, nicht direkt verwendbar. In erster Linie sind die ausgewiesenen Abschreibungen im Vergleich zu den unterstellten Kapitaleinsatzmengen der einzelnen Sektoren zu hoch angesiedelt, und die Darstellung des Staates seitens der amtlichen Statistik muß in einem Punkt der theoretischen Betrachtung angeglichen werden. Im Gegensatz zu den im letzten Abschnitt vorgenommenen Anpassungen können die hier auftretenden Diskrepanzen jedoch sukzessive angeglichen werden.

Die erste Modifikation ist noch als Konsequenz der Verteilung der Erträge aus unterstellter Produktion von Bankdienstleistungen in der Zentralmatrix zu betrachten. Denn durch die Umbuchung dieser Beträge auf die einzelnen Sektoren sind nun die Spaltensummen der Tabelle größer als die entsprechenden Zeilensummen. Es bietet sich an, die Differenz - in Anlehnung an die Vorgehensweise der amtlichen Statistik $^{96}$ - beim Nettobetriebsüberschuß ( $\approx$ Einkommen aus Unternehmertätigkeit und Vermögen) auszugleichen.

${ }^{96}$ Der Nettobetriebsüberschuß ergibt sich in der Volkswirtschaftlichen Gesamtrechnung als Saldo des Produktionskontos. Siehe hierzu bspw. STBA [1988b], S. 59 in Verbindung mit ZWER [1981], S. 33 f. zur Behandlung des Nettobetriebsüberschusses in der Volkswirtschaftlichen Gesamtrechnung. 
Der nächste Schritt befaßt sich mit der Erzeugung der „Kapitalsteuerzeile". Wie im theoretischen Teil erläutert wurde, setzt sich die Kapitalsteuer aus der Grundsteuer (A und B), der Gewerbesteuer, der Körperschaftsteuer und der Kapitalertragsteuer zusammen. Während Gewerbesteuern und Grundsteuern in der Position Produktionssteuern abzüglich Subventionen enthalten sind, müssen die übrigen Steuerarten dem Nettobetriebsüberschuß entnommen werden. Hierbei trifft man auf das gleiche Problem, das auch bei der Ermittlung der durchschnittlichen Umsatzsteuersätze auftrat: Die Inkompatibilität zwischen der funktionell ausgerichteten Input-Output-Tabelle und dem in der traditionellen Volkswirtschaftlichen Gesamtrechnung (VGR) verwendeten institutionellen Ausweis nach dem Schwerpunktprinzip machen eine direkte Umbuchung, unter Bezugnahme auf nach Wirtschaftsbereichen disaggregierten Steuerstatistiken unmöglich.

Als Alternative böte es sich an, die (direkte) Umbuchung in der nach Gütergruppen und institutionellen Wirtschaftsbereichen abgegrenzten Basistabelle (Make-Matrix) vorzunehmen und anschließend daraus analog zur Erstellungspraxis des Statistischen Bundesamtes ${ }^{97}$ - einen Wertschöpfungsquadranten in der gewünschten Aggregationstiefe abzuleiten. Allerdings ist dieser Prozeß sehr arbeitsintensiv und seinerseits mit größeren Unzulänglichkeiten verbunden, so daß die rein mechanische Tabellenerstellung durch manuelle Eingriffe, deren Kenntnis sich dem Verfasser entzieht, korrigiert werden muß. Darüber hinaus ist es generell fraglich, ob eine derartige an der Güterstruktur orientierte Überleitung den eher an institutionelle Kriterien - denkt man an die Gewerbesteuer auch an regionale - gebundenen Steuern gerecht wird.

Aus diesen Gründen wird eine zwar ebenso unzulängliche, aber weitaus unkompliziertere Arbeitshypothese unterstellt. Da die tatsächliche sektorale Verteilung der Steuern unbekannt ist, werden höhere Steuerbeträge dort vermutet, wo auch der entsprechende Felderwert der Input-Output-Tabelle einen hohen Betrag ausweist. Folglich werden die aus den jeweiligen Einzelstatistiken entnehmbaren gesamtwirtschaftlichen Steuerbeträge in der Regel proportional zu den einzelnen Felderwerten derjenigen Zeile subtrahiert, in der sie enthalten sind, und in einer Kapitalsteuerzeile zusammengefaßt.

${ }^{97}$ Siehe hierzu Stahmer [1984], S. 42 ff. oder STBA [1988b], S. 42. 
Die Grundsteuer A in Höhe von 450 Mill. $\mathrm{DM}^{98}$ wurde den Produktionssteuern des Sektors 1 (Land- und Forstwirtschaft), die Grundsteuer B in Höhe von insgesamt 6640 Mill. DM unter Berücksichtigung der $\mathrm{m}$. E. wichtigsten Steuerbefreiungen (geregelt in $\S 3 \mathrm{ff}$. GrStG) - öffentlicher Dienst, Bundesbahn, Sozialversicherungen, Organisationen ohne Erwerbszweck usw. den restlichen Produktionssteuerwerten entnommen.

Die Gewerbesteuer in Höhe von 28323 Mill. DM wurde ebenfalls proportional ohne Berücksichtigung der Land- und Forstwirtschaft, des Staates, der Krankenkassen, der Bundesbahn und Bundespost, der Organisationen o. E. , der Wissenschaft und des Gesundheitswesens ( $\$ 3 \mathrm{GewStG}$ ) aus der Produktionssteuerzeile entzogen und der neu entstandenen Zeile Kapitalsteuern hinzuaddiert.

Die Körperschaftsteuer mit einem gesamtwirtschaftlichen Aufkommen von rund 30300 Mill. $\mathrm{DM}^{99}$ vermindert in gleicher Weise den Nettobetriebsüberschuß, unter Berücksichtigung der in $\S 5 \mathrm{f}$. KStG geregelten bedeutsamsten Befreiungen, wie bspw. Bundespost, Bundesbahn, Krankenkassen.

Mit im wesentlichen den gleichen berücksichtigten Befreiungen (geregelt in $\S 44 \mathrm{ff}$. EStG) wie bei der Körperschaftsteuer wird auch die Kapitalertragsteuer in Höhe von 5591 Mill. DM ${ }^{100}$ proportional vom Nettobetriebsüberschuß subtrahiert und der Kapitalsteuerzeile beigefügt.

Die dritte Korrektur befaßt sich mit der Relation zwischen den Einkommen aus Unternehmertätigkeit und Vermögen und den Abschreibungen. Zum besseren Verständnis der vorzunehmenden Abstimmung muß etwas weiter ausgeholt werden. Theoretisch betrachtet, führen die Gleichgewichtspreise zur Räumung von Güter- und Faktormärkten. Man benötigt demnach zur Beschreibung eines Benchmark Equilibriums gesonderte Angaben über Mengen und Preise. Aus der InputOutput-Tabelle stehen jedoch nur Wertgrößen als Produkt von Menge mal Preis zur Verfügung. Zur Trennung beider Komponenten werden üblicherweise - so auch hier - die Mengeneinheiten einfach derart um-

\footnotetext{
${ }^{98}$ Die Daten für die Grundsteuern und der Gewerbesteuer sind aus STBA [1986b], S. 12 entnommen.

99 Aus STBA [1987b] S. 65.

100 Aus StBA [1987a], S. 424.
} 
definiert, daß der Preis einer Einheit gerade eine DM beträgt. Damit sind alle Produzentenpreise $q_{i}$ und die Netto-Faktorpreise $w$ und $r$ im Ausgangsgleichgewicht auf 1 normiert.

Als Konsequenz der Normierung repräsentiert die Zeile Einkommen aus Unternehmertätigkeit und Vermögen, welche im Modell die Entlohnung des Faktors Kapital $\left(r K_{i}, i=1, \ldots, 15\right)$ angibt, im Benchmark Equilibrium gleichzeitig auch den Kapitaleinsatz $\left(K_{i}\right)$ der Sektoren. Die Abschreibungen $\left(q^{I} D_{i}\right)$ werden ihrerseits durch den technologisch bedingten Verschleiß des eingesetzten Kapitals bestimmt, wobei der Verschleiß eine sektorspezifische, aber konstante Größe $\left(D_{i} / K_{i}\right)$ darstellt. Setzt man nun die in der Tabelle ausgewiesenen Abschreibungen $q^{I} D_{i}^{a}\left(\right.$ mit $\left.q^{I}=1\right)$ ins Verhältnis zu den Einkommen aus Unternehmertätigkeit und Vermögen $r K_{i}^{a}(\text { mit } r=1)^{101}$, erhält man teilweise Verschleißkoeffizienten von $150 \%$ und mehr. Eine Anpassung der Größen ist unumgänglich.

Andererseits soll jedoch die Relation zwischen ausgewiesenen Lohnund Kapitaleinkommen innerhalb eines Sektors - also $w L_{i}^{a} / r K_{i}^{a}-$ ebenso wie die relative Verteilung der Einkommensarten auf die einzelnen Sektoren $-w L_{i}^{a} / w L_{1}^{a}$ und $r K_{i}^{a} / r K_{1}^{a}$ (für $\left.i=1, \ldots, 15\right)$ - gewahrt bleiben. Aus diesem Grund wurden zunächst diese formal beschriebenen Verteilungskoeffizienten für die intersektorale Struktur der Lohnund Kapitaleinkommen gebildet und anschließend die Zeilensummen von Einkommen aus unselbständiger Arbeit $w L^{a}$ und Einkommen aus Unternehmertätigkeit und Vermögen $r K^{a}$ ins Verhältnis gesetzt. Es gilt

$$
V_{L}^{a}=\frac{w L^{a}}{r K^{a}}=\frac{L^{a}}{K^{a}} \quad w=r=1 .
$$

Analog bildet man die Relation zwischen gesamtwirtschaftlicher Abschreibung $D^{a}$ und dem gesamtwirtschaftlichen Anlagevermögen $\tilde{K}$ in Höhe von 5033200 Mill. DM ${ }^{102}$

$$
V_{A}^{a}=\frac{A^{a}}{\tilde{K}},
$$

da aus demselben Grund wie zuvor, nämlich den unterschiedlichen

101 Der Suffix $a$ kennzeichnet hierbei die ausgewiesenen Tabellengrößen.

102 Aus STBA [1985a], S. 320. 
Ausweisprinzipien, wiederum nur auf gesamtwirtschaftliche Größen zurückgegriffen werden kann.

Die Reskalierung der Größen erfolgt nun über die Definitionsgleichung für die Bruttowertschöpfung zu Faktorkosten, die - nach Ausklammern von $K-$ mit

$$
W_{f}=K\left(1+V_{A}+V_{L}\right)
$$

in eine Form gebracht werden, die eine Ausnutzung der oben ermittelten Verhältniszahlen erlaubt. Über die Zielgrößen $W_{f}^{a}, V_{L}^{a}$ und $V_{A}^{a}$ können zunächst der gesamtwirtschaftliche Kapitalstock und daran anschließend die gesamtwirtschaftlichen Abschreibungen und Lohneinkünfte reskaliert werden. Die eingangs ermittelten intersektoralen Verteilungskoeffizienten erlauben schließlich die Aufschlüsselung der neuen Aggregatsgrößen auf die einzelnen Sektoren, ohne dabei die vorgegebenen inter- und intrasektoralen Einkommensrelationen zu zerstören.

Als letzte Maßnahme ist das vom staatlichen Sektor 15 eingesetzte Kapital bzw. die Kapitalentlohnung einzufügen. Da für die Leistungen der Sozialversicherungen und der Gebietskörperschaften, die der Allgemeinheit ohne spezielles Entgelt zur Verfügung stehen $(\approx$ Staatsverbrauch), keine Marktpreise existieren, werden sie über die Aufwandseite bewertet. Dies hat zur Folge, daß weder in der traditionellen VGR noch in der Input-Output-Rechnung für den Staat ein Einkommen aus Unternehmertätigkeit und Vermögen ausgewiesen wird ${ }^{103}$. Das theoretische Modell unterstellt jedoch eine Art staatliches Unternehmen, welches das öffentliche Gut unter Einsatz von Zwischenprodukten, Arbeit und Kapital ,konkurrenzwirtschaftlich“ produziert. Das bedeutet, daß auch die vom Staat eingesetzten Primärfaktoren - insbesondere Kapital - zu den gleichgewichtigen Faktorpreisen entlohnt werden müssen.

Da die unterstellte Kapitalentlohnung im Ausgangsgleichgewicht gerade mit dem staatlichen Kapitalstock übereinstimmen muß, genügt es, das Anlagevermögen des Staates in Höhe von 417150 Mill. DM ${ }^{104}$ auf die neu gewonnenen staatlichen Abschreibungen nach dem obigen

${ }^{103}$ Siehe bspw. KopsCH [1983], S. 751.

${ }^{104}$ Aus STBA [1985a], S. 320. 
Tabelle 9: Wertschöpfung (in 100000000 DM)

\begin{tabular}{|c|rrrrr|r|}
\hline Sektor & \multicolumn{1}{c}{$w L_{i}$} & \multicolumn{1}{c}{$r K_{i}$} & $q^{I} D_{i}$ & \multicolumn{1}{c|}{$T_{i}^{k}$} & \multicolumn{1}{c|}{$T_{i}^{q}$} & $\sum$ \\
\hline 1 & 84.88 & 186.10 & 10.06 & 4.50 & -20.88 & 264.66 \\
2 & 290.34 & 215.61 & 14.54 & 36.06 & 15.46 & 572.01 \\
3 & 507.48 & 146.60 & 9.82 & 72.47 & 98.27 & 834.64 \\
4 & 859.91 & 124.92 & 7.89 & 10.82 & 3.65 & 1007.19 \\
5 & 498.45 & 73.97 & 7.32 & 12.21 & 5.17 & 597.12 \\
6 & 541.99 & 162.95 & 5.97 & 19.47 & 5.25 & 735.63 \\
7 & 277.96 & 72.89 & 4.48 & 9.81 & 3.26 & 368.40 \\
8 & 183.71 & 33.28 & 2.41 & 4.81 & 1.76 & 225.97 \\
9 & 249.62 & 142.48 & 5.84 & 60.90 & 113.82 & 572.66 \\
10 & 368.98 & 126.30 & 5.13 & 11.02 & 7.29 & 518.72 \\
11 & 922.54 & 836.60 & 27.19 & 70.91 & -59.39 & 1797.85 \\
12 & 420.94 & 908.06 & 24.66 & 102.54 & 24.38 & 1480.58 \\
13 & 520.95 & 96.86 & 7.05 & 0.07 & -0.02 & 624.91 \\
14 & 606.18 & 1596.85 & 63.94 & 187.91 & 60.65 & 2515.53 \\
15 & 914.90 & 391.06 & 11.78 & 0.00 & 2.60 & 1320.34 \\
\hline$\sum$ & 7248.83 & 5114.53 & 208.08 & 603.50 & 261.27 & 13436.21 \\
\hline
\end{tabular}

Symbole:

$\begin{array}{clcc}w & \text { Preis für Arbeit } & D_{i} & \text { Verschleiß in Sektor } i \\ r & \text { Preis für Kapital } & T_{i}^{k} & \text { Kapitalsteuer in } \\ L_{i} & \text { Arbeitseinsatz in } & & \text { Sektor } i \\ & \text { Sektor } i & T_{i}^{q} & \text { Produktionssteuer in } \\ K_{i} & \text { Kapitaleinsatz in } & & \text { Sektor } i \\ & \text { Sektor } i & & \end{array}$

Prinzip zu reskalieren. Mit diesem letzten Eingriff ist der Wertschöpfungsquadrant unseres Benchmark Equilibriums entwickelt. Die so gewonnenen Daten können Tabelle 9 entnommen werden. 


\section{Endnachfrage und Budgetrestriktionen}

Auch in der Endnachfragematrix sind relativ starke Veränderungen erforderlich. Denn neben den üblichen Angleichungen des Aussagegehalts einzelner Tabellenpositionen - bspw. der Anlageinvestitionen an die modellierten Sachverhalte müssen nun zusätzlich die von den Wirtschaftssubjekten einzuhaltenden Budgetrestriktionen eingebracht werden.

Die Ausgangstabelle unterscheidet spaltenweise in die Verwendungskategorien:

- Privater Verbrauch im Inland

- Staatsverbrauch

- Anlageinvestitionen

- Vorratsveränderungen

- Ausfuhr von Waren und Dienstleistungen.

Hinzu kommt die aufgrund des Ausweises der Importe nach Ursprungsbereichen entstehende - ursprünglich unterhalb der Matrix der Primärinputs aufgeführte - Korrekturzeile Einfuhr gleichartiger Güter zu AbZoll-Preisen, jetzt als Spalte mit negativem Vorzeichen. Zeilen- und Spaltensummen über die gesamte Tabelle hinweg geben somit die Produktionswerte je Sektor - d. h. das inländische Produktionsvolumen und nicht mehr, wie bisher, das gesamte inländische Güteraufkommen an ${ }^{105}$.

War zuvor in der Wertschöpfung eine gesonderte Zeile für die Kapitalsteuern zu erzeugen, so sind hier Vorratsveränderungen und Nettoinvestitionen Verwendungsarten, von denen im theoretischen Modell abstrahiert wird. Die Markträumungsbedingungen für Güter- und Faktormärkte wie auch die Budgetrestriktionen der Unternehmen (NullProfit Bedingungen) müssen folglich im Ausgangsgleichgewicht ohne Berücksichtigung dieser Verwendungskategorien erfüllt sein. Darüber hinaus ist das gesamtwirtschaftliche Exportvolumen der Summe der mit inländischen Preisen bewerteten Importe anzupassen und die Budgetrestriktion des Haushalts sowie die des Staates müssen eingehalten werden.

105 Diese Form der Darstellung wird bspw. von HoLUB/SCHNABL ([1985], S. 56) als Version A1 bezeichnet und ist dort näher erläutert. 
Bevor wir uns diesen Bedingungen zuwenden, werden zunächst die zur Reinvestition erforderlichen Gütermengen bestimmt. Hier wurde die Annahme getroffen, daß sich das Investitionsgut aus fixen Anteilen $\beta_{j}$ der für investive $Z$ wecke verwendeten Güterarten $j(j=1, \ldots, 14)$ zusammensetzt. Dieses Verhältnis $\beta_{j}=I_{j} / \sum_{j=1}^{14} I_{j}$ mit $I_{j}$ als der gesamtwirtschaftlichen Nachfrage nach Investitionsgut $j$ ist direkt aus der Input-Output-Tabelle zu ersehen. Für alle Sektoren gilt ferner die Beziehung, daß die Abschreibungen eines Sektors den Ausgaben für Reinvestitionen entsprechen. Da die Sektoren 1 bis 12 die auf Investitionsgüterkäufe anfallende Vorsteuer von ihrer Basis-Umsatzsteuerschuld absetzen können, bewerten sie ihre Abschreibungen zu Nettopreisen, das heißt, für sie gilt die Beziehung

$$
q^{I} D_{i}=\sum_{j=1}^{14} \beta_{j} q_{j} D_{i}=\sum_{j=1}^{14} q_{j} I_{j i} \quad i=1, \ldots, 12 .
$$

Aufgrund der Preisnormierung im Ausgangsgleichgewicht $\left(q_{j}=q^{I}=\right.$ $1 \forall i$ ) ergibt sich die von Sektor $i$ (mit $i=1, \ldots, 12)$ nachgefragte Menge $I_{j i}$ von Investitionsgut $j$ (mit $j=1, \ldots, 14$ ) - über obige Bedingung - unmittelbar aus den im vorangehenden Abschnitt entwickelten Abschreibungen.

Etwas aufwendiger ist die Behandlung der umsatzsteuerbefreiten Sektoren 13, 14 und 15. Da auch sie ihre Abschreibungen zu Wiederbeschaffungspreisen bewerten, muß aus den im Wertschöpfungsquadranten ausgewiesenen Größen $p^{I} D_{i}$ mit

$$
p^{I} D_{i}=\sum_{j=1}^{14} \beta_{j}\left(1+\tau_{j}^{u}\right) q_{j} D_{i} \quad i=13,14,15
$$

zunächst das reale Reinvestitionsvolumen $D_{i}$ bestimmt werden. Wie die obige Gleichung zeigt, führt eine Abdiskontierung von $p^{I} D_{i}$ mit $\sum_{j=1}^{14} \beta_{j}\left(1+\tau_{j}^{u}\right) q_{j}$ zu dem gewünschten Ergebnis. Die im Ausgangsgleichgewicht von Sektor 13,14 und 15 nachgefragten Reinvestitionsgütermengen sind wiederum über die Bedingung $I_{j i}=\beta_{j} D_{i}$ bestimmbar.

Faßt man die Reinvestitionsgüternachfrage der Sektoren nach Güterarten zusammen, so erhält man die Investitionsgüterspalte für das 
Ausgangsgleichgewicht. Die Differenz zwischen dieser Spalte und den tatsächlich ausgewiesenen Anlageinvestitionen wird in einem Zwischenschritt mit den Vorratsveränderungen zu einer Übergangsspalte zusammengefaßt. Sie repräsentiert somit die Abweichungen von Zeilen- und Spaltensummen im momentanen Entwicklungstadium unserer Datenbasis.

Die beiden Summen können auf zwei verschiedene Arten interpretiert werden: Erstens geben die Spalten die zur Produktion einer bestimmten Outputmenge notwendigen Inputkosten an, während die entsprechenden Zeilensummen die Netto-Verkaufserlöse quantifizieren. Die Tabelle kann deshalb als numerische Beschreibung der Null-ProfitBedingungen der Unternehmen aufgefaßt werden. Zweitens spezifizieren die Spaltensummen aufgrund der Preisnormierung die insgesamt produzierten (resp. angebotenen) Gütermengen, die Zeilensummen die insgesamt verbrauchten (resp. nachgefragten). Eine konsistente Tabelle beschreibt damit ebenfalls die Gütermarkt-Gleichgewichte. Unsere Übergangsspalte enthält also die Differenzbeträge, die für das Erreichen von ausgeglichenen Unternehmensbudgets und geräumten Gütermärkten noch umzubuchen sind.

Aus mehreren Gründen ist es nicht möglich, Ausgleichs-Algorithmen wie RAS oder MODOP heranzuziehen ${ }^{106}$. Beide dienen zwar prinzipiell eher zur "Schätzung“ von statistisch nicht fundierbaren Felderwerten, werden aber auch häufig zur Angleichung bestimmter Tabellenteile an theoretisch geforderte Restriktionen herangezogen ${ }^{107}$. In unserem Modell können jedoch nicht alle Bedingungen simultan einbezogen werden. Dies gilt in erster Linie für die endogen erzeugte Umsatzsteuerzeile $N U$ - jeder Eingriff in die Vorleistungsstruktur der letzten drei Sektoren, die Konsum- und die Investitionsgüterspalte würde die nichtabzugsfähige Umsatzsteuer implizit beeinflussen -, aber auch für diejenigen Budgetgleichungen, die übergreifend aus Elementen des II. und III. Quadranten bestehen.

Vor allem sind neben den Gütermarkt-Räumungsbedingungen und den Null-Profit-Bedingungen der Unternehmen auch die Budgets des

${ }^{106}$ Eine Beschreibung der Anwendungs- und Funktionsweise ist u. a. in HELMSTÄDTER [1983], S. $16 \mathrm{ff}$. zu finden.

107 Siehe u. a. KÜHN [1988] oder ST. Hilaire/Whalley [1983,1987]. 
Haushalts und des Staates in der Ausgangstabelle nicht erfüllt. Bedenkt man, daß weder Ersparnisse noch Einkommensteuern im modellierten Budget des repräsentativen Haushalts vorkommen - er verwendet sein gesamtes verfügbares Einkommen ausschließlich für Konsumausgaben -, kann dies kaum überraschen. Die Einkommensteuern, ebenso wie die modellmäßig unberücksichtigte Netto-Kreditaufnahme, bilden ihrerseits eine nicht unwesentliche Position auf der Ertragseite des tatsächlichen staatlichen Budgets. Beide Posten zusammengenommen machten im Jahr 1984 ungefähr 39\% der staatlichen Einnahmen aus ${ }^{108}$. Des weiteren erfordert das unterstellte AußenhandelsGleichgewicht die Nivellierung eines in der Input-Output-Tabelle 1984 auf rund 39 Milliarden DM bezifferten Netto-Exportüberschusses der Leistungsbilanz. Diese von der theoretischen Seite geforderten Restriktionen können nicht sinnvoll über ein mechanisches Ausgleichsverfahren erfüllt werden.

Ferner sind die Budgetgleichungen aller Wirtschaftssubjekte interdependent - d. h. die Erträge des einen sind gleichzeitig Teil der Aufwendungen eines oder mehrerer anderer -, so daß keine sukzessive Anpassung möglich ist. Vielmehr sind bei jeder Veränderung der Daten, bspw. in Richtung Ausgleich des Budgets des Haushalts, die dadurch entstehenden Veränderungen der Null-Profit-Bedingungen der Unternehmen und der Budgetgleichung des Staates zu berücksichtigen.

Aus diesen Überlegungen ist erkennbar, daß die Übergangsspalte nicht mit einer einzigen Verwendungskategorie - etwa dem Konsum - verrechnet werden kann. Jede Umbuchung hat vielmehr so zu erfolgen, daß sich die Salden der Budgetrestriktionen und der Leistungsbilanz verringern, wobei die Zeilen- und Spaltensummen der Tabelle nicht auseinanderfallen dürfen. An dieser Stelle sei nebenbei bemerkt, $\mathrm{da} ß$ alle ausgewiesenen Verwendungsarten staatlicher Leistungen (Sektor 15) in den Staatsverbrauch umgebucht wurden, da das öffentliche Gut weder international gehandelt wird, noch vom privaten Haushalt direkt erwerbbar ist.

Die manuelle Umbuchung der Übergangsspalte erfolgte deshalb im wesentlichen auf den Konsum, die Exporte oder die Importe, jeweils in Abhängigkeit von den entsprechenden ursprünglichen Größen-

${ }^{108}$ Aus ST BA [1987b], S. 20 und 65, ohne Berücksichtigung der Sozialbeiträge. 
Tabelle 10: Endnachfrage (in 100000000 DM)

\begin{tabular}{|c|rrrrr|r|}
\hline Sektor & \multicolumn{1}{c}{$q_{i} C_{i}$} & \multicolumn{1}{c}{$q_{15} G$} & \multicolumn{1}{c}{$q_{i} I_{i}$} & \multicolumn{1}{c}{$q_{i} X_{i}$} & \multicolumn{1}{c}{$q_{i} M_{i}$} & \multicolumn{1}{c|}{$\sum$} \\
\hline 1 & 211.86 & 0.00 & 0.10 & 40.72 & -401.91 & -149.23 \\
2 & 345.35 & 0.00 & 0.00 & 27.04 & -696.53 & -324.14 \\
3 & 640.53 & 0.00 & 0.59 & 864.85 & -880.01 & 625.96 \\
4 & 365.87 & 0.00 & 42.84 & 1356.03 & -755.11 & 1009.63 \\
5 & 657.85 & 0.00 & 12.03 & 772.34 & -266.07 & 1176.15 \\
6 & 635.93 & 0.00 & 23.70 & 641.41 & -494.62 & 806.42 \\
7 & 295.36 & 0.00 & 3.40 & 126.75 & -205.26 & 220.25 \\
8 & 556.97 & 0.00 & 0.24 & 196.24 & -393.94 & 395.51 \\
9 & 1398.30 & 0.00 & 0.00 & 180.68 & -346.71 & 1232.27 \\
10 & 286.79 & 0.00 & 97.45 & 330.97 & -7.95 & 707.26 \\
11 & 1703.46 & 0.00 & 10.24 & 387.73 & -181.20 & 1920.23 \\
12 & 452.38 & 0.00 & 5.54 & 458.88 & -100.80 & 816.00 \\
13 & 523.02 & 0.00 & 0.00 & 43.09 & -274.75 & 291.36 \\
14 & 2451.85 & 0.00 & 2.03 & 6.77 & -428.64 & 2032.01 \\
15 & 0.00 & 2934.73 & 0.00 & 0.00 & 0.00 & 2934.73 \\
\hline$\sum$ & 10525.52 & 2934.73 & 198.16 & 5433.50 & -5433.50 & 13658.41 \\
\hline $\mathrm{NU}$ & 895.80 & 0.00 & 9.92 & 0.00 & 0.00 & 1127.92 \\
\hline
\end{tabular}

Symbole:

$q_{i} \quad$ Produzentenpreis für Gut $i$

$C_{i} \quad$ Konsum von Gut $i$

$G \quad$ Bereitgestellte Menge des öffentlichen Gutes
$I_{i}$ Investition der Güterart $i$

$X_{i}$ Export von Gut $i$

$M_{i}$ Import von Gut $i$

$N U$ Nichtabzugsfähige

Umsatzsteuer

ordnungen. In wenigen Ausnahmefällen - hauptsächlich bei typischen Investitionsgüterarten (Sektor 4 und 10) - war eine Reskalierung der gesamten Tabellenspalte bzw. -zeile unumgänglich. Wurde die Umbuchung in den privaten Verbrauch vorgenommen, so waren die darauf anfallenden Umsatzsteuerbeträge zu berücksichtigen. Insgesamt wurde das ursprüngliche Verhältnis von nichtabzugsfähiger Umsatzsteuer im Produktionsbereich zum gesamtwirtschaftlichen Umsatzsteuer aufkommen beibehalten. Die relative Bedeutung der auf Investitionen lasten- 
den Umsatzsteuer konnte im Datensatz allerdings nicht gewahrt werden. Erfolgte eine Umbuchung zu den Exporten bzw. Importen, wurde darauf geachtet, daß die Netto-Handelsposition dieses Sektors nicht umschlug.

Die resultierende Endnachfragematrix ist in Tabelle 10 dargestellt. Damit sind alle notwendigen Basisdaten zur Beschreibung des Ausgangsgleichgewichts entwickelt. Der Budgetausgleich des Staates und des repräsentativen Haushalts wird über eine negative Transferzahlung in Höhe von 94204 Mill. DM erzielt. Dieser Wert ist nicht empirisch untermauert, sondern ergibt sich vielmehr aus der weiter oben beschriebenen gegenläufigen und extrem hohen Diskrepanz der jeweiligen Budgetseiten in der Ausgangssituation.

\section{Abschließende Bemerkungen}

Ziel dieser teilweise etwas eintönigen Schilderung der wesentlichen, zur Konstruktion einer mikro-konsistenten Datenbasis erforderlichen Eingriffe in das statistische Quellmaterial, sind vor allem Transparenzerwägungen. In zahlreichen Veröffentlichungen von EAG-Analysen wird der Leser mit einer durch Knappheit geprägten Darstellung der numerischen Seite konfrontiert, die jegliche Versuche eines Nachvollziehens von vornherein ausschließen. Zum Teil wird die Datenbasis einfach konstatiert oder aus Platzgründen gar nicht erst angegeben. Auch die vorliegende Darstellung ist - wie eingangs erwähnt - keine protokollartige Schilderung der durchgeführten Modifikationen; dennoch erlaubt sie m. E. durch das Aufzeigen der wesentlichen Problempunkte und deren Lösungsansatz ein gewisses $M a ß$ an Nachvollziehbarkeit und liefert gleichzeitig Orientierungshilfen für weitere Arbeiten in dieser Richtung.

Ferner kann dadurch die Aussagekraft der ansonsten als gegeben präsentierten Daten besser eingeschätzt werden. Ein Punkt, der auch im Hinblick auf die später ermittelten Wohlfahrtseffekte eine gewisse Rolle spielt, da Effizienzberechnungen nicht nur von mehr oder weniger stringenten theoretischen Verhaltensannahmen, sondern ebenso vom numerischen Ausgangsgleichgewicht abhängen.

Wie aus den vorangehenden Erläuterungen hervorging, mußten mitunter heroische Umbuchungen vorgenommen werden, um die Daten mit 
den theoretischen Implikationen in Einklang zu bringen. Schon allein diese Entfernung von den tatsächlich ausgewiesenen Daten verbietet es, allzusehr an der numerischen Ausprägung der Effizienzberechnungen festzuhalten. Dies soll nun nicht bedeuten, daß die Ergebnisse im Grunde völlig willkürlich sind, sondern daß auch den errechneten numerischen Werten eine mehr qualitative Bedeutung zukommt. 


\section{Teil C: Kalibrierung und Wahl der exogenen Parameter}

Das Empirische Allgemeine Gleichgewichtsmodell ist komplett, wenn auch für die exogenen Parameter der im theoretischen Teil abgeleiteten Verhaltensfunktionen numerische Werte bestimmt sind. Hier liegt das Vorgehen nahe, die Parameter mit Hilfe geeigneter Verfahren ökonometrisch zu schätzen. In den wenigsten Modellen wird jedoch tatsächlich ein ökonometrischer Ansatz verwendet ${ }^{109}$. Das Standardverfahren zur Festlegung der exogenen Parameter besteht vielmehr aus der teilweisen Übernahme zentral erscheinender Parameterwerte aus der ökonometrischen Literatur und einer mit „Kalibrierung“ umschriebenen Methode. Bei letzterer werden die Parameter gerade so festgelegt, daß die entsprechenden Verhaltensfunktionen den (im vorangehenden Teil B bestimmten) vorgegebenen Gleichgewichtswert annehmen.

Als einfaches Beispiel dazu könnte man die Berechnung der InputKoeffizienten über die Beziehung $a_{i j}=A_{i j} / Q_{j}$ nennen, wobei $A_{i j}$ und $Q_{j}$ aus der Datenbasis bekannt sind. Mit etwas gutem Willen wäre die Kalibrierung folglich auch als Ein-Punkt-Schätzverfahren zu bezeichnen. Dennoch ist es sicherlich nicht überraschend, daß dieses Vorgehen einen wesentlichen Kritikpunkt an Empirischen Allgemeinen Gleichgewichtsmodellen eröffnet. Wir wollen allerdings nicht zu sehr auf die Diskussion über Stärken und Schwächen der Kalibrierung gegenüber ökonometrischen Verfahren eingehen ${ }^{110}$. Aufgrund der zentralen Bedeutung dieser Kritik seien dennoch die wichtigsten Argumente kurz erläutert.

109 Eine Ausnahme hierzu bildet bspw. JoRGENSON [1984,1985].

110 Siehe hierzu u. a. PIggott/Whalley [1985]. 


\section{Kalibrierung oder ökonometrischer Ansatz}

Der Hauptkritikpunkt liegt auf der Hand. Statistisch gesehen impliziert der Kalibrierungsansatz, daß nur diejenigen Einflußfaktoren die endogenen Variablen bestimmen, die im Modell explizit vorkommen. Die in ökonometrischen Ansätzen durch die stochastischen Störterme repräsentierten unsystematischen Einflußgrößen werden von vornherein ausgeschlossen. Abgesehen von dieser sehr deterministischen Betrachtungsweise, die - bedenkt man das Abstraktionsniveau der Modelle für sich selbst spricht, sind damit auch keinerlei statistische Signifikanztests u. ä. anwendbar, mit denen die empirische Relevanz der so bestimmten Parameterwerte (und auch der Erklärungswert der unterstellten funktionalen Formen) abgeschätzt werden kann. Es gibt folglich keine Anhaltspunkte für die Verlässlichkeit der kalibrierten Parameterwerte.

Allerdings stößt auch eine ökonometrische Schätzung von Gleichgewichtsmodellen walrasianischer Bauart auf Schwierigkeiten. Bedingt durch ihre Modellstruktur ist es meist nicht möglich, alle Parameter im ökonometrischen Sinne zu identifizieren. Da die beobachtbaren Größen Gleichgewichtswerte darstellen, kann aus der betreffenden Zeitreihe der Verlauf der Angebots- und Nachfragekurven nicht eindeutig bestimmt werden. Abbildung 1 dient zur Illustration des Problems. Die Punktewolke repräsentiert hierbei die beobachteten Preis-Mengen Realisationen für einen beliebigen Markt. Die verschiedenen Beobachtungen könnten sowohl aus einer Veränderung der Angebots-, als auch der Nachfragekurve für diesen Markt zustande gekommen sein. Ob nun die durchgezogenen Angebots- und Nachfragekurven S bzw. D oder die gestrichelten Linien $\mathrm{S}^{\prime}$ und $\mathrm{D}^{\prime}$ den tatsächlichen Funktionsverlauf besser charakterisieren kann auch statistisch nicht ohne weiteres festgestellt werden.

Erst durch die Erweiterung des Modells um eine zusätzliche exogene Variable, die nur das Niveau einer der beiden Funktionen beeinflußt, kristallisiert sich - wie in Abbildung 2 angedeutet - der Verlauf der jeweils anderen Funktion heraus. Durch die Hinzunahme von exogenen Variablen würde aber das Modell vor allem in Hinblick auf die damit durchgeführten Besteuerungssimulationen wesentlich an seiner Attraktivität verlieren, die ja gerade von der Geschlossenheit des Systems 
Abbildung 1

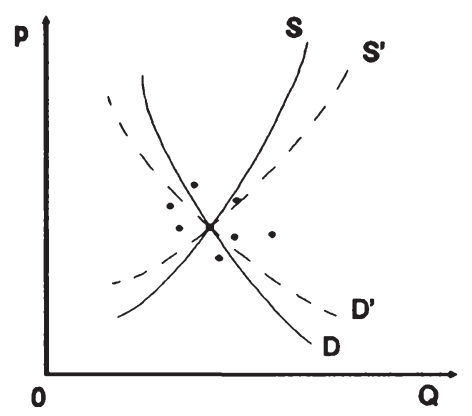

Abbildung 2

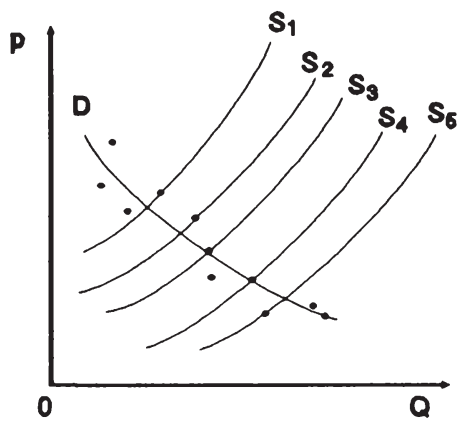

ausgeht.

Schätzt man auf der anderen Seite nicht das gesamte Modell simultan, sondern in mehrere Blöcke unterteilt, so kann durch die Vernachlässigung systematischer Einflußfaktoren ein simultaneous equation bias entstehen ${ }^{111}$. In diesem Fall erfaßt der Störterm nicht nur zufällige Schwankungen, sondern auch einen systematischen Einfluß, was sich in den geschätzten Parametern niederschlägt. Beispielsweise mag die in Abbildung 3 dargestellte Punktewolke der beobachteten Werte zur (isolierten) Schätzung einer Nachfragekurve einladen. Da die Beobachtungen im Grunde jedoch Gleichgewichtswerte repräsentieren (oder zumindest zum Gleichgewicht hin tendieren), trifft die Schätzgerade (gestrichelte Linie) nicht den tatsächlichen Funktionsverlauf (durchgezogene Linie).

Mit diesen relativ globalen Anmerkungen sind natürlich nur die grundsätzlichen Probleme angesprochen, die bei der ökonometrischen Schätzung eines walrasianischen Gleichgewichtsystems auftauchen ${ }^{112}$.

${ }^{111}$ Für nähere Erläuterungen siehe bspw. KOUTSOYANNIS [1981], S. $331 \mathrm{ff}$.

112 Spezifischere Argumente sind u. a. aus MANSUR/WHALLEY [1984] zu ersehen, wobei jedoch erwähnt werden sollte, daß manche, der dort angeführten ökonometrischen Probleme - wie bspw. das Auftreten von wechselseitigen Abhängigkeiten in den stochastischen Termen - nur bei Verwendung ungeeigneter Schätzverfahren auftreten (vgl. LAU [1984]). 


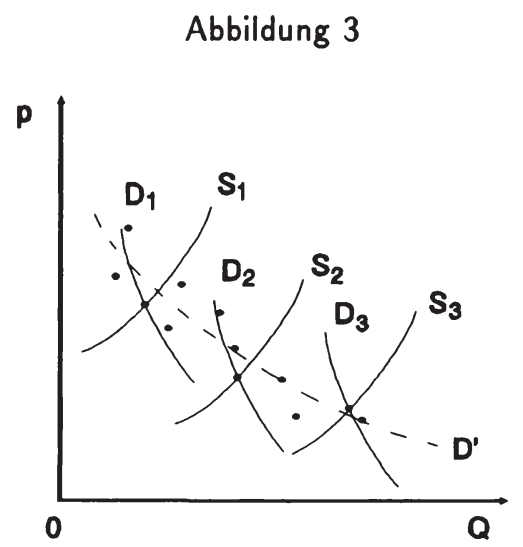

Außerdem kann mit dem Hinweis auf derlei Probleme nicht die Verwendung des Kalibrierungsansatzes begründet werden. Auch letzterer ist nicht imstande, das Identifikationsproblem zu "lösen“, sondern er schiebt es nur beiseite.

Darüber hinaus können nicht alle Parameter bestimmter Funktionstypen auf eine einzige Beobachtung hin kalibriert werden. Deshalb wird beispielsweise bei den unterstellten CES-Wertschöpfungsfunktionen der Wert der Substitutionselastizität $\sigma_{i}$ aus entsprechenden ökonometrischen Arbeiten übernommen und der Gewichtungsparameter $\rho_{i}$ kalibriert. Da den herangezogenen ökonometrischen Quellen in der Regel ein auf einzelne Gleichungen angewandtes Schätzverfahren zugrundeliegt, ist auch das mit Abbildung 3 beschriebene Problem einer verzerrten Schätzung der Kalibrierung immanent.

Der eigentliche Grund, weshalb in EAG-Modellen fast ausnahmslos der Kalibrierungsansatz zur Anwendung kommt, ist vielmehr in der beträchtlich geringeren Menge an Daten zu sehen, die zur Bestimmung der Parameter benötigt werden. Nicht selten bildet die Zusammenstellung eines einzigen Ausgangsgleichgewichts als Datenbasis des Modells den arbeitsintensivsten Teil der gesamten EAG-Analyse. Für eine ökonometrische Schätzung müßten ganze Zeitreihen erstellt werden, wodurch zusätzliche Probleme - wie etwa die (zeitabhängige) Normierung aller Gleichgewichtspreise - entstehen. „The major advantages 
of the calibration approach consists of minimal data requirements - one observation will do - and minimal computation requirements relative to the econometric approach. Thus, it is ideally suited to situations in which data are scarce and a quick answer is required", um mit den Worten von LAU ([1984], S. 136) zu schließen.

In den folgenden beiden Abschnitten wird nun die Kalibrierung der Parameter für die unterstellten Nachfrage- bzw. Angebotsfunktionen der Unternehmen und des Haushalts näher erläutert. Den Abschluß bildet eine Aufstellung aller aus der Literatur entlehnten und kalibrierten Parameterwerte.

\section{Unternehmensbereich}

Auf der Unternehmenseite sind folgende Größen zu bestimmen: die Produktion- und Kapitalsteuersätze $\tau_{i}^{q}$ bzw. $\tau_{i}^{k}$, die Verschleißkoeffizienten $\delta_{i}$ sowie die Koeffizienten $\beta_{i}$ der Umwandlungstechnologie für Investitionsgüter in Kapital, die Input-Koeffizienten $a_{j i}$ der Produktionsfunktionen und schließlich die Parameter der CES-Wertschöpfungsfunktionen $\sigma_{i}, \rho_{i}$ und $\Phi_{i}$.

Die Steuersätze können unmittelbar aus der Datenbasis gewonnen werden. Da die Produktion- und die Kapitalsteuer als einfache ad valorem Steuern - ohne Berücksichtigung von Freibeträgen und dgl. - modelliert sind, ergeben sich die Steuersätze aus der Relation zwischen den jeweiligen Steuerbeträgen und der entsprechenden Bemessungsgrundlage mit

$$
\tau_{i}^{k}=\frac{T_{i}^{k}}{r K_{i}} \quad \text { bzw. } \quad \tau_{i}^{q}=\frac{T_{i}^{q}}{q_{i} Q_{i}} \quad i=1, \ldots, 15
$$

Ebenso direkt lassen sich die Input-Koeffizienten und die Parameter der Investitions-Technologie bestimmen. Aus der Datenbasis sind die Einsatzmengen an Zwischenprodukten $A_{j i}$ und die Höhe der Wertschöpfung $f^{i}\left(K_{i}, L_{i}\right)$ für jedes Unternehmen $i$ bekannt. Setzt man diese Werte ins Verhältnis zum Gesamtoutput $Q_{i}$, erhält man mit $a_{j i}=A_{j i} / Q_{i}(i, j=1, \ldots, 15)$ und $a_{0 i}=f^{i}\left(K_{i}, L_{i}\right) / Q_{i}$ die benötigten 
Input-Koeffizienten. Analog werden die Parameter $\beta_{j}$ der InvestitionsTechnologie über die Relation zwischen (gesamtwirtschaftlicher) Nachfrage $I_{j}$ nach Investitionsgut $j$ und der Spaltensumme $\sum_{j=1}^{14} I_{j}$ ermittelt.

Bei der Berechnung der produktionstechnisch bedingten Verschleißkoeffizienten ist wiederum zu beachten, daß die Sektoren 13,14,15 im Ausgangsgleichgewicht umsatzsteuerbefreit sind. Die ausgewiesenen Abschreibungen $p^{I} D_{i}$ (mit $\left.i=13,14,15\right)$ müssen deshalb zunächst auf die Mengenkomponente reduziert werden. Da alle Produzentenpreise im Ausgangsgleichgewicht auf Eins normiert sind, erhalten wir den Verschleiß $D_{i}$ über die Division der Abschreibungen durch $p^{I}=\sum_{j=1}^{14} \beta_{j}\left(1+\tau_{j}^{u}\right) q_{j}$, wobei die Umsatzsteuersätze ebenfalls aus dem Datensatz bekannt sind. Für die Sektoren 1-12 ist wegen $q^{I}=1$ keine derartige Preisbereinigung notwendig. Liegen die $D_{i}$ vor, dann können die Verschleißkoeffizienten $\delta_{i}$ für alle Sektoren über $\delta_{i}=D_{i} / K_{i}$ ermittelt werden.

Die Parameter $\sigma_{i}, \rho_{i}$ und $\Phi_{i}$ der CES-Wertschöpfungsfunktion sind hingegen nicht alle aus der Datenbasis allein bestimmbar. Wir werden deshalb die Substitutionselastizitäten $\sigma_{i}$ zwischen Arbeit und Kapital aus den Ergebnissen einer von HEubes [1969] für die Bundesrepublik durchgeführten ökonometrischen Schätzung übernehmen. Mit gegebenen $\sigma_{i}$ kann über die aus dem Kostenminimierungsproblem vorsteuerabzugsberechtigter Unternehmen abgeleiteten Bedingung für den optimalen Faktoreinsatz

$$
\frac{r\left(1+\tau_{i}^{k}\right)+\delta_{i} q^{I}}{w}=\frac{1-\rho_{i}}{\rho_{i}}\left(\frac{L_{i}}{K_{i}}\right)^{\frac{1}{\sigma_{i}}} \quad i=1, \ldots, 12
$$

der Gewichtungsparameter $\rho_{i}$ berechnet werden. Löst man die obige Gleichung nach $\rho_{i}$ auf, ergibt sich

$$
\begin{gathered}
\rho_{i}=\frac{\Gamma_{i}}{1+\Gamma_{i}} \\
\operatorname{mit} \quad \Gamma_{i}=\frac{w}{\left(1+\tau_{i}^{k}\right) r+\delta_{i} q^{I}}\left(\frac{L_{i}}{K_{i}}\right)^{\frac{1}{\sigma_{i}}} .
\end{gathered}
$$


Die Bestimmungsgleichungen der für die umsatzsteuerbefreiten Sektoren $13,14,15$ relevanten Gewichtungsparameter sind hierzu identisch, nur daß anstelle von $q^{I}$ der Bruttopreis $p^{I}$ zu setzen ist.

Als letzter Schritt werden die Effizienzparameter $\boldsymbol{\Phi}_{i}$ so determiniert, daß die CES-Wertschöpfungsfunktionen bei Vorgabe der nun bekannten Parameter den in der Datenbasis ausgewiesenen Wert $a_{0 i} Q_{i}$ annehmen. Sie dienen, technisch betrachtet, als Skalierungsfaktoren, die die beobachtete Wertschöpfung mit dem Funktionswert in Einklang bringen.

\section{Haushaltsbereich}

Ähnlich zur Unternehmensseite sind auch für den repräsentativen Haushalt die Parameter einer CES-Funktion - hier definiert über das Composite-Commodity Konsum $C$ und die Freizeit $F$ - zu bestimmen. Hinzu kommen die aus dem Cobb-Douglas Nutzenast stammenden fixen Ausgaben anteile $\alpha_{i}$ der Konsumgüter-Nachfragefunktionen und die nicht beobachtbare gesamte Ausstattung an Zeit $E$.

Die Ausgabenanteile können direkt aus der Datenbasis ermittelt werden. Mit den dort ausgewiesenen Größen $w L=\sum_{i=1}^{15} w L_{i}$ und $r \bar{K}=\sum_{i=1}^{15} r K_{i}$ sowie den (negativen) Transfers $T R$ steht auch das verfügbare Einkommen $Y^{\text {ver }}$ des Haushalts fest. Die Brutto-Konsumausgaben $\left(1+\tau_{i}^{u}\right) q_{i} C_{i}$ folgen ebenfalls unmittelbar aus den Umsatzsteuersätzen und den ausgewiesenen Netto-Ausgaben $q_{i} C_{i}$. Damit kann durch einfache Umformung der Konsumgüter-Nachfragefunktionen der jeweilige Ausgabenanteil über

$$
\alpha_{i}=\frac{p_{i} C_{i}}{Y^{\text {ver }}} \quad i=1, \ldots, 14
$$

determiniert werden.

Etwas aufwendiger gestaltet sich die Berechnung der Substitutionselastizität $\nu$ zwischen Freizeit und globalem Konsum $C$, zumal letzterer eine rein fiktive Größe darstellt, für die keine ökonometrischen Schätzungen auffindbar sind. Was jedoch aus der Literatur vorliegt, sind Schätzungen der unkompensierten Arbeitsangebotselastizität $e$ 
bezüglich des Lohnsatzes. Wir werden deshalb zunächst eine Beziehung zwischen der Substitutionselastizität $\nu$ und der unkompensierten Freizeitnachfrageelastizität $\hat{e}$ bezüglich des Lohnsatzes aufstellen und anschließend $\hat{e}$ mit $e$ miteinander verbinden.

Die unkompensierte Freizeitnachfrageelastizität ist durch

$$
\hat{e}:=\frac{\partial F}{\partial w} \frac{w}{F}
$$

definiert. Leitet man die Freizeitnachfragefunktion

$$
F=\frac{\theta Y^{m a x}}{\Omega w^{\nu}}
$$

mit $\Omega=(1-\theta) p^{1-\nu}+\theta w^{1-\nu}$

demgemäß nach $w$ ab, ergibt sich

$$
\frac{\partial F}{\partial w}=\frac{\theta E}{\Omega w^{\nu}}-\frac{(1-\nu) \theta^{2} Y^{\max }}{\Omega^{2} w^{\nu}}-\frac{\nu \theta w^{-\nu-1} Y^{\max }}{\Omega} .
$$

Dieser Ausdruck kann in die Definitionsgleichung für die Freizeitnachfrageelastizität eingesetzt werden. Man erhält:

$$
\hat{e}=\frac{w E}{Y^{\max }}-\frac{(1-\nu) \theta w^{1-\nu}}{\Omega}-\nu .
$$

In einem Zwischenschritt definieren wir $s_{e}:=(w E) / Y^{\max }$ als Wertanteil der gesamten Zeitausstattung am maximalen Einkommen und entsprechend $s_{f}$ als Wertanteil der Freizeit, wobei $s_{f}$ durch Einsetzen der Freizeit-Nachfragefunktion auch über

$$
s_{f}:=\frac{w F}{Y^{\max }}=\frac{\theta w^{1-\nu}}{\Omega}
$$

beschrieben werden kann.

Verwendet man diese Definitionen in der Bestimmungsgleichung für $\hat{e}$, folgt

$$
\hat{e}=s_{e}-(1-\nu) s_{f}-\nu,
$$


woraus durch Auflösen nach $\nu$ mit

$$
\nu=\frac{-\hat{e}+s_{e}-s_{f}}{1-s_{f}}
$$

eine handliche Beziehung zwischen der Substitutionselastizität und der unkompensierten Freizeitnachfrageelastizität herstellbar ist.

Den letzten Schritt bildet das Ersetzen von $F$ durch $E-L$ in der Definitionsgleichung der Freizeitnachfrageelastizität $\hat{e}$. Auf diese Weise kann über

$$
\begin{aligned}
\hat{e} & =\frac{\partial(E-L)}{\partial w} \frac{w}{E-L} \\
& =-\frac{\partial L}{\partial w} \frac{w}{E-L}
\end{aligned}
$$

wegen $\partial E / \partial w=0$, die Verbindung zur Arbeitsangebotselastizität $e$ verdeutlicht werden. Definieren wir $\xi:=E / L$, läßt sich $\hat{e}$ durch

$$
\hat{e}=-e \frac{1}{\xi-1}
$$

bestimmen. Da aus der Datenbasis nur das gleichgewichtige Arbeitsangebot, der gesamtwirtschaftliche Kapitalstock sowie die Faktorpreise bekannt sind, müssen $e$ und $\xi$ bzw. $E$ vorgegeben werden, um die Substitutionselastizität $\nu$ berechnen zu können.

Der Gewichtungsparameter $\theta$ folgt aus der Division der FreizeitNachfragefunktion $L$ durch die Konsum-Nachfragefunktion $C$, wobei letztere durch

$$
C=\frac{(1-\theta) Y^{\max }}{\Omega p^{\nu}}
$$

gegeben ist. Man erhält aus

$$
\frac{F}{C}=\frac{\theta}{1-\theta} \frac{p^{\nu}}{w^{\nu}}
$$

nach einigen Umformungen den Ausdruck 


$$
\theta=\left[\frac{w^{\nu} F}{p^{\nu} C}\right] /\left[1+\frac{w^{\nu} F}{p^{\nu} C}\right] .
$$

Mit der Bestimmung von $\theta$ sind alle Parameter der im Modell verwendeten Funktionen determiniert. Sie werden im folgenden Abschnitt zusammen mit den exogen gesetzten Parameterwerten tabellarisch aufgeführt.

\section{Die Parameterwerte im einzelnen}

Als exogene Parameter sind die Substitutionselastizitäten $\sigma_{i}$, die Arbeitsangebotselastizität $e$ sowie die gesamte Zeitausstattung $E$ des repräsentativen Konsumenten und schließlich die Außenhandelselastizitäten $\Theta$ und $\eta$ vorzugeben.

Tabelle 11 zeigt die exogen spezifizierten Substitutionselastizitäten $\sigma_{i}$ zwischen Arbeit und Kapital sowie die für die Wertschöpfungsfunktionen abgeleiteten Parameter. Für die vorzugebenden Substitutionselastizitäten konnte auf die ökonometrischen Studien von HEUBES [1969] und Roskamp [1977] für die Bundesrepublik Deutschland zurückgegriffen werden, wobei die Schätzungen von RosKAMP tendenziell etwas höhere Elastizitätswerte ergaben. Wie im vorangehenden Abschnitt bereits erwähnt, wurde den Schätzungen von Heubes der Vorzug gegeben, da sie aus einem längeren Beobachtungszeitraum resultieren. Allerdings untersuchten beide nur Industriezweige. Der gesamte Dienstleistungsbereich ebenso wie die land- und forstwirtschaftlichen Betriebe blieben außer Betracht. Für letztere konnte zumindest aus einer australischen Untersuchung von CADDY [1976 ${ }^{113}$ der Wert von 0.8 entlehnt werden. Wohl aufgrund des spärlichen statistischen Zahlenmaterials wurde jedoch auch in dieser Arbeit der Dienstleistungsbereich nicht miteinbezogen.

Als Anhaltspunkt für die Spezifizierung der Substitutionselastizitäten im Dienstleistungsbereich diente der von HEuBEs festgestellte $\mathrm{Zu}$ sammenhang, daß die Substitutionselastizität tendenziell in den Wirtschaftszweigen hoch ist, die eine vergleichsweise hohe Kapitalintensität

${ }^{113}$ Entnommen aus PIggotT/Whalley [1985], S. $160 \mathrm{f}$. 
Tabelle 11: Parameter der Wertschöpfungsfunktion

\begin{tabular}{|c|cccc|}
\hline Sektor & $\sigma_{i}$ & $\rho_{i}$ & $\Phi_{i}$ & $a_{0 i}$ \\
\hline 1 & 0.4 & 0.1153 & 1.7680 & 0.3802 \\
2 & 0.5 & 0.5949 & 2.2467 & 0.4246 \\
3 & 0.8 & 0.7515 & 2.3255 & 0.2830 \\
4 & 0.4 & 0.9908 & 1.2904 & 0.2861 \\
5 & 0.7 & 0.9235 & 1.4860 & 0.3494 \\
6 & 0.4 & 0.9458 & 1.5955 & 0.4443 \\
7 & 0.9 & 0.7872 & 1.7925 & 0.3550 \\
8 & 0.4 & 0.9833 & 1.3891 & 0.3475 \\
9 & 0.7 & 0.6027 & 2.9139 & 0.2805 \\
10 & 0.5 & 0.8833 & 1.7211 & 0.4989 \\
11 & 0.9 & 0.4994 & 2.0468 & 0.4894 \\
12 & 1.2 & 0.3161 & 2.0574 & 0.6336 \\
13 & 0.4 & 0.9842 & 1.3708 & 0.7721 \\
14 & 1.3 & 0.2908 & 2.1089 & 0.6874 \\
15 & 0.5 & 0.8416 & 1.9175 & 0.4275 \\
\hline
\end{tabular}

Symbole:
$\sigma^{i} \quad$ Substitutionselastizität
$\Phi^{i} \quad$ Effizienzparameter von Arbeit zu Kapital
$\rho_{i} \quad$ Gewichtungsparameter
$a_{0 i} \quad$ Anteil der Wertschöpfung an der Produktionsfunktion

$K_{i} / L_{i}$ aufweisen. Dieses Vorgehen ist sicherlich nicht befriedigend. Wir werden deshalb im nächsten Kapitel untersuchen, wie sensitiv die Wohlfahrtseffekte auf Veränderungen der Substitutionselastizität reagieren.

Im ersten Teil von Tabelle 12 sind die Verschleißkoeffizienten der Sektoren und die Koeffizienten der Investitions-Technologie angegeben. Der zweite Teil zeigt die Kapital- und Produktionssteuersätze des Ausgangsgleichgewichts. Die teilweise negativen Produktionssteuersätze sind darauf zurückzuführen, daß die entsprechenden Sektoren netto subventioniert werden ${ }^{114}$. Da alle ausgewiesenen Größen unmit-

114 Siehe hierzu Teil A Teilabschnitt (2.2) dieses Kapitels. 
Tabelle 12: Parameter der Investitionstechnologie und Steuersätze

\begin{tabular}{|c|cc|cc|}
\hline Sektor & $\delta_{i}$ & $\beta_{i}$ & $\tau_{i}^{k}$ & $\tau_{i}^{q}$ \\
\hline 1 & 0.0541 & 0.0005 & 0.0242 & -0.0300 \\
2 & 0.0674 & 0.0000 & 0.1672 & 0.0115 \\
3 & 0.0670 & 0.0030 & 0.4943 & 0.0333 \\
4 & 0.0632 & 0.2162 & 0.0866 & 0.0010 \\
5 & 0.0990 & 0.0607 & 0.1651 & 0.0030 \\
6 & 0.0366 & 0.1196 & 0.1195 & 0.0032 \\
7 & 0.0615 & 0.0172 & 0.1346 & 0.0031 \\
8 & 0.0724 & 0.0012 & 0.1445 & 0.0027 \\
9 & 0.0410 & 0.0000 & 0.4274 & 0.0557 \\
10 & 0.0406 & 0.4918 & 0.0872 & 0.0070 \\
11 & 0.0325 & 0.0517 & 0.0847 & -0.0162 \\
12 & 0.0272 & 0.0280 & 0.1129 & 0.0104 \\
13 & 0.0641 & 0.0000 & 0.0007 & -0.0000 \\
14 & 0.0352 & 0.0102 & 0.1177 & 0.0160 \\
15 & 0.0265 & 0.0000 & 0.0000 & 0.0008 \\
\hline
\end{tabular}

Symbole:

$\delta_{i} \quad$ Verschleißkoeffizient für Unternehmen $i$

$\beta_{i} \quad$ Investitionskoeffizient für Investitionsgut $i$

$\tau_{i}^{k} \quad$ Kapitalsteuersatz

$\tau_{i}^{q} \quad$ Produktionsteuersatz

telbar aus der Datenbasis hervorgehen, bedürfen sie wohl keiner weiteren Erläuterung.

Auf Haushaltsebene wird - in Ermangelung spezifischer Angaben für die Bundesrepublik - bei der Festlegung des Verhältnisses $E / L$ und der Wahl der unkompensierten Arbeitsangebotselastizität $e$ bezüglich des Lohnsatzes dem Vorgehen von Ballard/Fullerton/Shoven/ Whalley ([1985], S. 135 ff.) gefolgt. Nimmt man an, daß die durchschnittliche Arbeitszeit 40 Stunden pro Woche beträgt, wobei der Haushalt maximal 70 Stunden pro Woche arbeiten könnte, so ist $\xi$ auf 1.75 fixiert. Durch die Wahl eines Mittelwertes von $e=0.15$ wird ferner unterstellt, daß der Haushalt auf Lohnsatzerhöhungen mit einer Stei- 
gerung des Arbeitsangebotes reagiert. Die aus dieser Spezifizierung kalibrierten Parameter sind in Tabelle 13 aufgeführt.

Tabelle 13: Kalibrierte Parameter im Haushaltsbereich

\begin{tabular}{|c|c|c|c|c|}
\hline CES-Nutzenast & \multicolumn{4}{|c|}{ Cobb-Douglas-Nutzenast } \\
& Gut & $\alpha_{i}$ & Gut & $\alpha_{i}$ \\
\hline$\nu=0.9299$ & 1 & 0.0199 & 8 & 0.0556 \\
$\theta=0.3614$ & 2 & 0.0342 & 9 & 0.1316 \\
& 3 & 0.0630 & 10 & 0.0286 \\
& 4 & 0.0365 & 11 & 0.1684 \\
& 5 & 0.0657 & 12 & 0.0437 \\
& 6 & 0.0633 & 13 & 0.0458 \\
& 7 & 0.0291 & 14 & 0.2146 \\
\hline
\end{tabular}

Symbole:

$\nu$ Substitutionselatizität zwischen Freizeit und Konsum

$\theta$ Gewichtungsparameter

$\alpha_{i} \quad$ Ausgabenanteil für Konsumgut $i$

Für die Außenhandelselastizitäten wurden schließlich - in Anlehnung an HiRTE/WiEgaRd [1988] - die Werte $\vartheta=-10.0$ und $\eta=0.12$ exogen vorgegeben. Diese Parameterkombination steht in Einklang mit der in Stern, Francis und Schumacher ([1976], S. 16) als 'best guess' aufgeführten (gleichgewichtigen) Exportnachfrageelastizität von -1.11 und impliziert, daß die Exporte relativ stark auf Eigenpreisveränderungen reagieren. Die für die Außenhandelsfunktionen vorzugebenden fixen Export- bzw. Import-Gütermengen $X_{i}^{0}$ und $M_{i}^{0}$ können direkt aus der Datenbasis übernommen werden.

Mit der numerischen Konkretisierung der Parameter ist nun auch der letzte Arbeitsgang in der Entwicklung eines Empirischen Allgemeinen Gleichgewichtsmodells abgeschlossen. Sicherlich ist die Gewinnung der Parameterwerte sehr deterministisch und bietet berechtigten Anlaß zur Skepsis. Darüber hinaus zeigen verschiedene ökonometrische Schätzungen eines bestimmten Parameters kein so einheitliches Bild, 
wie es wünschenswert wäre ${ }^{115}$. Folglich ist auch die Wahl eines konkreten Parameterwertes aus der ökonometrischen Literatur mit einiger Willkür behaftet. Wir werden deshalb die im nächsten Kapitel vorgenommenen Steuerreform-Simulationen mit unterschiedlichen $\mathrm{Pa}$ rametervorgaben berechnen, um beurteilen zu können, inwiefern die Modellergebnisse durch die Wahl der Parameter beeinflußt werden. Die oben aufgelisteten Parameterwerte bilden jedoch die sog. zentrale oder Standard-Parameterwahl.

${ }^{115} \mathrm{Da}$ ökonometrische Schätzergebnisse u. a. von der Aufbereitung des statistischen Datenmaterials und dem verwendeten Schätzverfahren abhängen, kann dies nicht überraschen. Einen Eindruck für die Verschiedenheit der numerischen Resultate liefert bspw. ein Überlick von KILLINGSWORTH [1983] über mehrere Schätzungen der unkompensierten Arbeitsangebotselastizität bezüglich des Lohnsatzes. 


\section{Kapitel IV}

\section{Steuersimulationen}

Die zuerst durchgeführte Simulation kann noch zu den Vorbereitungen im weiteren Sinne gezählt werden. Nachdem das im vorangehenden Kapitel beschriebene Modell auf einem Rechner implementiert ist, wird mit dem Replication Check die Konsistenz der Programmierung und des Modells überprüft. Bei Vorgabe der aus der Datenbasis bekannten bzw. ermittelten Steuersätze muß sich über den Lösungsalgorithmus das Benchmark Equilibrium exakt replizieren lassen. Wird dieser Probelauf erfolgreich beendet, ist das Modell für weitere Simulationsläufe bereit. Das heißt, man verändert eine oder mehrere staatliche Variablen - im allgemeinen Besteuerungsregelungen - und berechnet hierzu ein neues Gleichgewicht. Ein Vergleich dieses neuen oder Counterfactual Equilibrium mit dem Ausgangsgleichgewicht als Referenz ermöglicht dann die Einschätzung der simulierten Steuerreform.

Bevor wir jedoch durch Veränderung der staatlichen Variablen alternative Gleichgewichte berechnen, erscheinen einige technische Bemerkungen über EAG-Analysen sinnvoll, da sie zum besseren Verständnis der nachfolgenden Simulationsläufe beitragen. Sie betreffen den Modellablauf - genauer gesagt, die Abfolge in der die ökonomischen Größen numerisch determiniert werden -, die Ausgestaltung der in diesem Rahmen betrachtbaren Steuerreformen und schließlich die ökonomische Beurteilung der simulierten Steuerreform.

\section{Vorbemerkungen}

\subsection{Der Modellablauf}

Läßt man staatliche Aktivitäten zunächst außer Betracht, kann der Lösungsweg zur Determinierung der Modellvariablen folgendermaßen dargestellt werden: Ausgangspunkt sind die Faktorpreise $r$ und $w$. Ein konkretes Faktorpreisverhältnis legt die Faktoreinsatzverhältnisse von Kapital und Arbeit im Unternehmensbereich fest. Aufgrund der Homogenität der Wertschöpfungsfunktionen ändern sich letztere nur bei 
variierenden Faktorpreisen, aber nicht bei Veränderung des jeweiligen Unternehmensoutputs. Somit kann die Faktornachfrage $k_{i}$ und $l_{i}$ pro Einheit Output der Unternehmen sowie deren Kapitalverschleiß pro Einheit Output bestimmt werden. Erstere determinieren ihrerseits über die entsprechenden Null-Profit-Bedingungen - die Güterpreise ${ }^{116}$. Da die Güterpreise in die Kapitalnutzungskosten der Unternehmen mit einfließen, müssen Einheitsfaktornachfrage und Güterpreise genau genommen simultan bestimmt werden.

Für gegebene Güterpreise und gegebenen Lohnsatz kann anschlieBend auf der Haushaltsseite das Arbeitsangebot und die Konsumgüternachfrage ermittelt werden. Darüber hinaus legen die Güterpreise das Importangebot und die Exportnachfrage des Auslands fest. Zusammen mit den Produktionsfunktionen - d. h. der daraus hervorgehenden Nachfrage nach Vorleistungen - bestimmen diese Endnachfragekomponenten den Unternehmensoutput insgesamt, wobei Unternehmensoutput und die Nachfrage der Unternehmen nach Investitionsgütern wiederum simultan zu berechnen sind. Mit dem Output steht schließlich die absolute Faktornachfrage jedes einzelnen Unternehmens und damit auch die gesamtwirtschaftliche Nachfrage nach Kapital und Arbeit fest.

Die gesamtwirtschaftliche Faktornachfrage wird nun mit dem Faktorangebot des Haushalts verglichen und führt, entsprechend der sich einstellenden Überschußnachfragen, zu einer Veränderung der Faktorpreise. Mit den geänderten Faktorpreisen wird das System dann erneut durchlaufen, bis letztendlich die Uberschußnachfragen den Wert Null annehmen.

Diese kurze Beschreibung des Modellablaufs zeigt, daß nicht alle Gleichgewichtspreise simultan bestimmt werden müssen, sondern nur die beiden Faktorpreise alle maßgeblichen Größen sukzessive determinieren. Bei Hinzunahme staatlicher Aktivitäten erhöht sich das zu lösende simultane Gleichungssystem um eine Dimension. Eine staatliche Variable wird endogen über den Lösungsalgorithmus bestimmt, wobei die staatliche Budgetgleichung als weitere Überschußnachfragefunktion dient. Demzufolge ist bei jeder untersuchten Besteuerungs-

${ }^{116}$ Durch die Ausklammerung von staatlichen Aktivitäten erübrigt sich (momentan) die Unterscheidung zwischen Produzenten- und Konsumentenpreisen. 
variation die endogene Anpassung mindestens einer staatlichen Budgetposition erforderlich. Diese Anpassung, die letztendlich zu einem gesamtwirtschaftlichen Gleichgewicht - insbesondere zu einem ausgeglichenen staatlichen Budget - führt, muß jedoch nicht aus der endogenen Bestimmung eines einzelnen Steuersatzes oder einer bestimmten Ausgabenposition bestehen, sondern sie kann auch eine allgemeine Anhebung bestimmter Steuersätze (in additiver oder multiplikativer Form) darstellen.

\subsection{Budget- und Differentialinzidenz}

Im Zusammenhang mit den geplanten Steuersimulationen erhebt sich somit automatisch die Frage, welche staatliche Größe endogen über den Lösungsalgorithmus bestimmt werden soll, damit die resultierenden allokativen Effekte analytisch zugeordnet werden können. Prinzipiell gibt es hierbei zwei Möglichkeiten: Man berechnet eine Budgetinzidenz ${ }^{117}$, bei der die Veränderung bestimmter Steuersätze durch eine Anpassung der Ausgabenseite des staatlichen Budgets aufgefangen wird, oder man berechnet eine Differentialinzidenz, bei der die Ausgabenseite konstant gehalten wird und der Budgetausgleich ausschließlich durch Anpassung der Einnahmeseite erfolgt.

Der eingangs des Kapitels erwähnte Replication Check stellt beispielsweise eine Budgetinzidenz dar. Werden alle aus der Datenbasis bekannten Steuersätze direkt vorgegeben, dann muß der Lösungsalgorithmus bei Konstanz der bereitgestellten Menge des Kollektivgutes - neben den gleichgewichtigen Faktorpreisen - die gleichgewichtigen staatlichen Transferleistungen bestimmen. Da die von der Umsatzsteuer - insbesondere die von unechten Umsatzsteuerbefreiungen - ausgehenden Zusatzlasten im Mittelpunkt unseres Interesses stehen, werden jedoch in den nachfolgenden Abschnitten hauptsächlich Differentialinzidenzen durchgeführt. Denn bei gleichzeitiger Variation von Einnahme- und Ausgabenseite, wie es bei Budgetinzidenzen der Fall ist, kann die resultierende Wohlfahrtsänderung nicht ausschließlich der veränderten Steuersatzstruktur zugeordnet werden, sondern beinhaltet

${ }^{117}$ Der von MUSGRAVE geprägte Begriff einer Balanced Budget Incidence ist hierzu gleichbedeutend. Die Betonung des ausgeglichenen Budgets erübrigt sich jedoch im Rahmen unserer Allgemeinen Gleichgewichtsanalyse. 
ebenfalls Wohlfahrtseffekte aufgrund eines erhöhten oder verminderten staatlichen Ausgabenniveaus.

Die Forderung einer konstanten Ausgabenseite bzw. die Erfüllung dieser Forderung ist jedoch eine nicht ganz unproblematische Angelegenheit ${ }^{118}$. Durch die Änderung bestimmter Steuern werden üblicherweise Reaktionen der Wirtschaftssubjekte ausgelöst, die zu veränderten relativen Preisen führen. Ein nominell konstanter Anteil der Ausgaben für das öffentliche Gut könnte nun möglicherweise nicht mehr dazu ausreichen, dieselbe Bereitstellungsmenge wie im Benchmark Equilibrium zu gewährleisten. Bedenkt man, daß die Nutzenstiftung von Gütern ausgeht, haben auch nominell konstante staatliche Transferzahlungen im neuen Gleichgewicht einen anderen Stellenwert als im Ausgangszustand, da mit ihnen je nachdem nun mehr bzw. weniger Güter erworben werden können.

Aus diesem Grund halten wir die reale Ausgabenseite konstant. Die Bereitstellungsmenge des Kollektivgutes wird auf das Niveau des Ausgangsgleichgewichts fixiert und die neuen Transferzahlungen über einen Laspeyres-Preisindex ihrer Kaufkraft im Benchmark Equilibrium angeglichen. Es sei jedoch angemerkt, daß der Laspeyres-Preisindex $P_{L}$, mit

$$
P_{L}=\frac{\sum_{i} p_{i}^{1} C_{i}^{0}}{\sum_{i} p_{i}^{0} C_{i}^{0}},
$$

wobei die Indizes 0,1 die alten bzw. neuen Größen repräsentieren, die Preisänderung tendenziell überschätzt. Da das Mengengerüst des Ausgangsgleichgewichts konstant bleibt, werden die durch Veränderung der relativen Konsumgüterpreise ausgelösten Substitutionseffekte des Haushalts nicht berücksichtigt, sodaß der Haushalt nicht in vollem Umfang von der im Index zum Ausdruck kommenden Kaufkraftänderung getroffen wird. Auf der anderen Seite bereitet die Implementierung eines alternativen Preisindex - bspw. ein Index nach Paasche -, der diese Substitutionseffekte miteinbezieht, Probleme im Ablauf des Modells ${ }^{119}$.

${ }^{118}$ Siehe hierzu auch KITTERER [1985], S. $78 \mathrm{ff}$.

${ }^{119} \mathrm{Zu}$ dem Zeitpunkt, zu dem die Transfers über den Preisindex anzupassen sind, kann die veränderte Konsumgüternachfrage noch nicht bestimmt werden. Nähere Erläuterungen siehe bspw. SHOVEN/WHALLEY [1977]. 


\subsection{Lösungsalgorithmus und Eindeutigkeit des Gleichge- wichts}

Der Ursprung der computerunterstützten Allgemeinen Gleichgewichtsanalyse ist in den Arbeiten von SCARF $[1967,1973]$ über die Berechnung ökonomischer Gleichgewichte und den darauf folgenden steuerpolitischen Anwendungen von Shoven und Whalley [1972,1973] zu sehen. Während mit den Fixpunktsätzen von BrouWER oder KaKUTANI die Existenz eines Allgemeinen Gleichgewichts feststellbar ist, ermöglicht der von ScARF entwickelte Algorithmus das Auffinden dieses Gleichgewichts. Er kann somit als konstruktiver Existenzbeweis für ein Allgemeines Gleichgewicht betrachtet werden (vgl. TaKayama [1986], S. 260). In der vorliegenden Arbeit wurde die von MERRILL [1972] verbesserte Version des Algorithmus verwendet, deren Vorteil hauptsächlich in einer vergleichsweise kürzeren Rechenzeit zur Auffindung des Fixpunktes besteht ${ }^{120}$.

Noch kürzere Rechenzeiten sind unter Umständen mit sog. QuasiNewton-Methoden - wie sie häufig in Programmbibliotheken wie IMSL oder NAG angeboten werden - oder mit Black-Box-Algorithmen - wie etwa PLALGO von M. Todd oder OVTASOLVE von M. Broadie - erreichbar. Allerdings haben diese Verfahren den Nachteil, daß die Konvergenz des Startpunktes zum Gleichgewicht von mehreren Faktoren - darunter auch der Wahl des Startpunktes selbst - abhängt und nicht allgemein garantiert ist.

Prinzipiell stellt die numerische Berechnung eines Gleichgewichtspunktes mittlerweile kein größeres Problem mehr dar. Allerdings ist die auf einem Vergleich von Referenzzustand und neuem Gleichgewicht basierende komparative Statik wertlos, falls kein eindeutiges Gleichgewicht existiert, sondern mehrere Gleichgewichte nebeneinander bestehen. Hier wurden zwar von KеноE [1980,1985a] Index-Theoreme entwickelt, die die notwendige und hinreichende mathematische Bedingung für Eindeutigkeit angeben, welche Beschränkungen für die Parameter des ökonomischen Modells diese Bedingungen implizieren, ist jedoch

${ }^{120}$ Für eine Beschreibung des in diesen Algorithmen angewandten Lösungsverfahrens siehe u. a. CORNWALL [1984], mehr anwendungsorientierte Erläuterungen sind bspw. in WIEGARD [1985] zu finden. 
bislang ungeklärt. Hinzu kommt, daß auch der Lösungsalgorithmus nur jeweils einen gleichgewichtigen Preisvektor pro Simulationslauf berechnet, selbst wenn mehrere Gleichgewichte nebeneinander existieren sollten.

Eine Möglichkeit zur Feststellung multipler Gleichgewichte besteht darin, den Algorithmus mit unterschiedlichen Startpunkten beginnen zu lassen. Konvergiert er immer zum selben Gleichgewicht hin, so läßt sich Eindeutigkeit vermuten. Praktische Erfahrungen ${ }^{121}$ haben jedoch gezeigt, daß der Algorithmus nur dann zu einem zweiten Gleichgewicht konvergiert, wenn der Startpunkt bereits sehr nahe an diesem Gleichgewicht liegt. Ist dies nicht der Fall, wird auch mit unterschiedlichen Startpunkten immer dasselbe Gleichgewicht gefunden.

Eine zweite Möglichkeit bietet das von КЕноE/Whalley [1985] gewählte Verfahren. Man reduziert das simultane Lösungsproblem von drei auf zwei Dimensionen, wobei sichergestellt wird, daß die eliminierte Überschußnachfrage eine eindeutige Lösung besitzt. Die verbleibenden beiden Überschußnachfragen werden anschließend für unterschiedliche Preisvorgaben berechnet und in ein Diagramm abgetragen. Falls diese geplotteten Funktionen nur einen einzigen Schnittpunkt mit der NullKoordinate aufweisen, ist die Eindeutigkeit des Gleichgewichts sichergestellt. Wir werden diese Vorgehensweise für die zentrale Parameterspezifizierung unseres Modells anhand der oben beschriebenen Budgetinzidenz - d. h. alle Steuersätze sind numerisch vorgegeben und die Transferzahlungen werden über den Algorithmus bestimmt - nachvollziehen.

Allgemein können die drei ursprünglichen Überschuß-Nachfragefunktionen durch folgendes Gleichungssystem beschrieben werden:

$$
\pi_{L}=\sum_{i=1}^{15} L_{i}(w, r, T R)-L(w, r, T R)
$$

121 Siehe bspw. KEHOE [1985b]. 


$$
\begin{aligned}
& \pi_{K}=\sum_{i=1}^{15} K_{i}(w, r, T R)-\bar{K} \\
& \pi_{S t}=q_{15}(w, r) \bar{G}+T R-T(w, r, T R) .
\end{aligned}
$$

Aus den Erläuterungen zum Ablauf des Modells ging hervor, daß die Faktorpreise das Faktoreinsatzverhältnis und damit die Produzentenpreise bestimmen, während die Transfers über die einkommensabhängige Konsumgüternachfrage des Haushalts nur die (absolute) Höhe der Faktornachfrage der Unternehmen beeinflussen. Erhöht man nun die Transferzahlungen, steigt cet. par. die Überschußnachfrage des Staates $\pi_{S t}$. Da alle Steuersätze zwischen Null und Eins liegen, kann das gesamtwirtschaftliche Steueraufkommen $T(w, r, T R)$ nicht um denselben Betrag steigen wie die Transferzahlungen. Folglich steigt $\pi_{S t}$ monoton in $T R$, was bedeutet, daß zu jeder Faktorpreis-Kombination ein eindeutiger Wert für $T R(w, r)$ zu einem ausgeglichenen staatlichen Budget führt.

Setzt man diesen Wert in die Überschuß-Nachfragefunktionen für Arbeit und Kapital ein, so reduziert sich das Lösungsproblem

$$
\begin{array}{ll}
\pi_{L}=\sum_{i=1}^{15} L_{i}(w, r, T R(w, r))-L(w, r, T R(w, r)) & =0 \\
\pi_{K}=\sum_{i=1}^{15} K_{i}(w, r, T R(w, r))-\bar{K} & =0
\end{array}
$$

auf zwei Dimensionen. Des weiteren können die Faktorpreise in diesem reduzierten System aufgrund der Nullhomogenität der Angebots- und Nachfragefunktionen bezüglich der Faktorpreise auf $w+r=1$ normiert werden. In Abbildung 4 sind die Überschuß-Nachfragefunktionen $\pi_{K}$ und $\pi_{L}$ in Abhängigkeit von $w$ (womit auch $r=1-w$ determiniert ist) gezeichnet. Beide Funktionen weisen im relevanten Preisintervall nur einen gemeinsamen Schnittpunkt auf. Damit ist zumindest für die zentrale Parametersetzung Eindeutigkeit des Gleichgewichts sichergestellt. 
Abbildung 4: Die ÜberschuB-Nachfragefunktionen

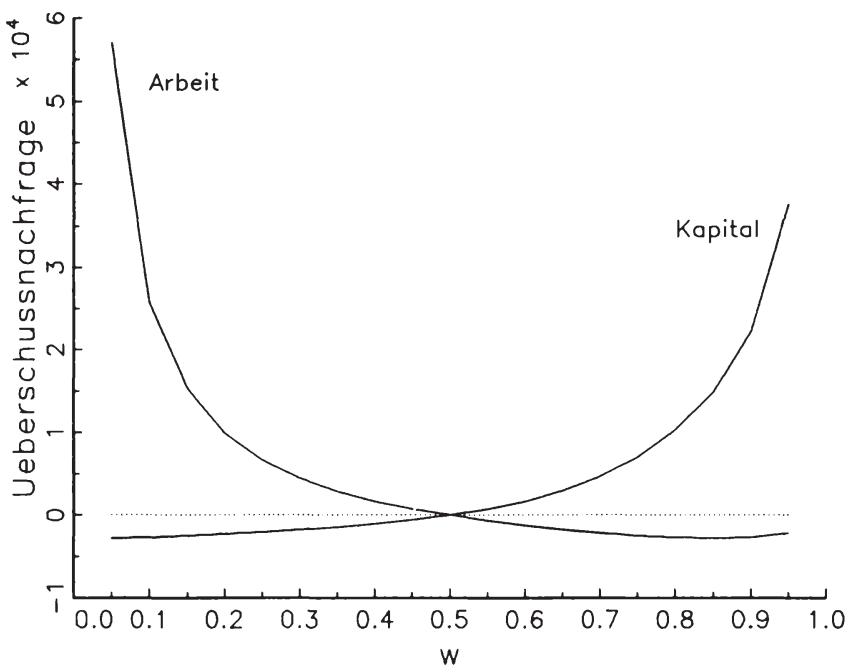

Die Tatsache, daß die Überschuß-Nachfragefunktionen die Null-Koordinate im Punkt $w=r=0.5$ schneiden, während für den eigentlichen Replication-Check ein Gleichgewichtspunkt von $w=r=1$ ermittelt wurde, ist dadurch zu erklären, daß bei letzterem alle Gleichgewichtspreise (einschließlich der Transferzahlungen) auf den Lohnsatz normiert sind. Diese Normierung auf den Lohnsatz wird, nebenbei bemerkt, bei allen weiteren Simulationsläufen - auch den Differentialinzidenzen beibehalten.

\subsection{Die ökonomische Beurteilung der Steuerreform}

Als letzte Vorbemerkung ist noch zu klären, anhand welcher Maßzahl ein durch bestimmte Steuerreformen entstandenes alternatives Gleichgewicht mit dem Benchmark Equilibrium verglichen werden soll. Prinzipiell wäre es möglich, alle Änderungen der Faktor- und Gütermengen zu ermitteln. In der Regel beschränkt man sich bei der Einschätzung 
der Effizienz- und Verteilungswirkung jedoch auf die Berechnung einiger gesamtwirtschaftlicher Kennziffern. Da wir darüber hinaus nur die Effizienz der Besteuerung betrachten - Verteilungseffekte sind schon durch die Unterstellung eines repräsentativen Konsumenten ausgeschlossen -, begnügen wir uns mit der Berechnung eines einzelnen monetären Indikators: der Äquivalenten Variation von HicKs.

Die Äquivalente Variation $E V$ ist definiert als

$$
\begin{aligned}
E V & =\varrho\left(\mathbf{p}_{\mathbf{0}} ; \mathbf{p}_{\mathbf{1}}, Y_{1}\right)-\varrho\left(\mathbf{p}_{\mathbf{0}} ; \mathbf{p}_{\mathbf{0}}, Y_{0}\right) \\
& =\varrho\left(\mathbf{p}_{\mathbf{0}} ; \mathbf{p}_{\mathbf{1}}, Y_{1}\right)-Y_{0}
\end{aligned}
$$

mit $\mathbf{p}_{\mathbf{0}}, \mathbf{p}_{\mathbf{1}}$ als den Preisvektoren des Ausgangs- bzw. neuen Gleichgewichts und $Y_{0}, Y_{1}$ als den entsprechenden Einkommensgrößen ${ }^{122}$. Die Kompensationsfunktion $\varrho\left(\mathbf{p}_{\mathbf{0}} ; \mathbf{p}_{\mathbf{1}}, Y_{1}\right)$ gibt dabei an, wieviel Einkommen der repräsentative Haushalt bei den Preisen po $_{0}$ benötigen würde, um dieselbe Nutzenstiftung wie bei den Preisen $\mathbf{p}_{1}$ und dem Einkommen $Y_{1}$ zu erzielen. Dementsprechend mißt die Äquivalente Variation den bei den Preisen $\mathbf{p}_{0}$ benötigten, das ursprüngliche Einkommen $Y_{0}$ „übersteigenden“ Einkommensbetrag, der das Erreichen des im Counterfactual Equilibrium realisierten Nutzenniveaus ermöglicht. Eine positive $E V$ bedeutet somit eine Wohlfahrtsverbesserung, eine negative $E V$ entsprechend eine Verschlechterung ${ }^{123}$.

In Anhang 4 wird gezeigt, daß sich die oben beschriebene allgemeine Definition der Äquivalenten Variation für die von uns gewählte funktionale Form der Nutzenfunktion auf die handliche Formel

$$
E V=\frac{U_{1}-U_{0}}{U_{0}} Y_{0}^{\max }
$$

122 Siehe bspw. VARIAN [1985], S. 271.

${ }^{123}$ Demgegenüber kommt in typischen EAG-Analysen häufig auch die Kompensierte Variation zur Anwendung. Da sie jedoch für die Berechnung der Einkommensäquivalente im Gegensatz zur EV den jeweils neuen Preisvektor p1 zugrundelegt, erscheint sie vor allem bei der Beurteilung mehrerer alternativer Steuerreformen weniger geeignet, weil die gemeinsame Vergleichsbasis fehlt (vgl. auch AHLHEIM/ROSE [1984] oder EBERT [1988]). 
bringen läßt, wobei die realisierten Nutzenindizes $U_{0}$ bzw. $U_{1}$ jeweils nur den ersten Summanden der Nutzenfunktion $U(C, F)+H(G)$ repräsentieren. Wir wollen bei allen Simulationen die Bereitstellungsmenge des Kollektivgutes $G$ konstant halten, sodaß dem repräsentativen Haushalt keine Nutzenänderung aufgrund einer veränderten Menge des Kollektivgutes widerfährt.

\section{Der Übergang zu Nullsteuersätzen}

Nach all diesen Vorbemerkungen können wir endlich mit der Simulation der für unsere Fragestellung - der Effizienz von Umsatzsteuerbefreiungen - zentralen Steuerreform ${ }^{124}$ beginnen. Wird den im Benchmark-Gleichgewicht von der Umsatzsteuer befreiten und nicht vorsteuerabzugsberechtigten Unternehmen 13,14 und 15 nun ebenfalls ein Vorsteuerabzugsrecht eingeräumt, so reduziert sich das Umsatzsteueraufkommen um rund $21.0 \%$, falls die ursprünglichen Umsatzsteuersätze beibehalten werden. Dieses Ergebnis ist insofern interessant, weil der Anteil der nicht auf Konsum lastenden nichtabzugsfähigen Umsatzsteuer im Ausgangsgleichgewicht lediglich $19.9 \%$ betrug. Es zeigt sich somit, daß der Ausfall an Steueraufkommen größer ist, als zunächst vermutet wird.

Für die Beurteilung der Effizienz verschiedener Steuersatzstrukturen sind derartige Aufkommenseffekte jedoch zu vermeiden, da sie auf das Einkommensniveau des Haushalts rückwirken. Deshalb werden in der nun folgenden Simulation - einer Differentialinzidenz die ursprünglichen Umsatzsteuersätze um einen skalaren Faktor $d$ mit $\tau_{i, \text { neu }}^{u}=\tau_{i, \text { alt }}^{u} \cdot d \forall i$ angehoben, um das gesamtwirtschaftliche Steueraufkommen auf einem real konstanten Niveau zu halten. Die multiplikative Erhöhung der alten Steuersätze stellt dabei sicher, daß zumindest die im Ausgangsgleichgewicht bestehende Größenrelation der Steuersätze untereinander erhalten bleibt.

Die Steuerreform führt zu einer positiven Äquivalenten Variation in Höhe von 155 Mill. DM. Damit können Befürworter von Nullsteuersätzen - wie bspw. CNOSSEN [1981] - zunächst bestätigt werden. Al-

124 Siehe hierzu auch GotTfRIED/WIEGARD [1990], S. 15 ff. 
lerdings ist der in Einkommensäquivalenten gemessene Wohlfahrtsgewinn relativ moderat. Er beträgt nur rund $1.5 \mathrm{v}$. T. des insgesamt erzielten Umsatzsteueraufkommens. Die ökonomische Erklärung dieses Effizienzgewinns ist allerdings einigermaßen komplex und kann nicht ohne weiteres mit dem bloßen Hinweis auf die Reduktion der allokativen Verzerrungen abgetan werden, die zuvor durch die unechten Umsatzsteuerbefreiungen ausgelöst wurden.

Zur Ableitung einer besseren ökonomischen Interpretation wollen wir deshalb vier weitere Differentialinzidenzen durchführen, mit denen der aufgetretene Wohlfahrtseffekt näher aufschlüsselbar ist. Als besonders hilfreich erweist sich dabei die Anwendung des in Kapitel II entwickelten Konzepts der effektiven Steuersätze. Dort wurde herausgestellt, daß zur Beurteilung der allokativen Effekte nicht die nominellen Umsatzsteuersätze, sondern die effektiven Steuersätze herangezogen werden sollten, falls einige der Unternehmen umsatzsteuerbefreit und nicht vorsteuerabzugsberechtigt sind. In der im letzten Abschnitt von $\mathrm{Ka}$ pitel II angegebenen Tabelle 4 wurden die das Ausgangsgleichgewicht charakterisierenden effektiven Steuersätze aufgelistet.

Bezüglich der effektiven Steuersätze hat der allgemeine Übergang von unechten Befreiungen zu Nullsteuersätzen zur Folge, daß sich im neuen Gleichgewicht die Struktur der effektiven Konsumsteuersätze verändert - sie entspricht nun den nominellen Umsatzsteuersätzen - und die von den Befreiungen ausgelöste indirekte Besteuerung der Investitionen und des Außenhandels gänzlich entfällt. An diese Überlegung sind die nachfolgenden vier Simulationen angelehnt. Der oben errechnete Gesamteffekt wird gewissermaßen in einzelne Komponenten zerlegt. Tabelle 14 zeigt die Idee dieser Zerlegung.

In einem geeignet modifizierten Modell, das eine Veränderung der einzelnen Steuerarten erlaubt, wird die eigentlich simultan stattfindende Änderung der effektiven Besteuerung nun schrittweise simuliert. Die erste Simulation (in Tabelle 14 als Nr. 1 bezeichnet) verifiziert zunächst, daß die ursprünglichen effektiven Steuersätze auch tatsächlich das Ausgangsgleichgewicht erzeugen. In der zweiten Differentialinzidenz werden nun die ursprünglichen effektiven Außenhandelund Investitionsteuersätze (absolut) vorgegeben und nur die Struktur der Konsumsteuersätze - d. h. das Verhältnis der Konsumsteuersätze 
Tabelle 14: Der sukzessive Übergang von Befreiungen zu Nullsteuersätzen

\begin{tabular}{|c|c|c|c|r|r|}
\hline & \multicolumn{3}{|c|}{ Effektive Steuersätze auf } & & \\
Nr. & Konsum & Außenhandel & Investitionen & $E V^{*}$ & $\Delta E V^{*}$ \\
\hline 1 & alt & alt & alt & 0 & 0 \\
2 & neu & alt & alt & -1121 & -1121 \\
3 & neu & 0 & alt & 208 & 1329 \\
4 & neu & 0 & 0 & 155 & -53 \\
\hline
\end{tabular}

* in Millionen DM

zueinander - der veränderten Situation angepaßt. Danach folgt sukzessive die Abschaffung der Steuer auf Außenhandel (Differentialinzidenz Nr. 3) und Investitionen (Differentialinzidenz Nr. 4). Aus der letzten Simulation geht schließlich wieder das mit der zentralen Umsatzsteuerreform erzeugte und durch die Nullsteuersätze charakterisierte Counterfactual Equilibrium hervor.

Die in den letzten beiden Spalten von Tabelle 14 aufgelisteten Werte der Äquivalenten Variation geben die erzielten Wohlfahrtseffekte an, wobei $E V$ die Wohlfahrtsänderung im Vergleich zum Benchmark Equilibrium und $\Delta E V$ die Änderung in Bezug auf das mit der jeweils vorangehenden Simulation errechnete Gleichgewicht darstellt. Es zeigt sich, daß die größten Effizienzwirkungen von der Änderung der Konsumund der Außenhandelsteuer ausgehen. In den folgenden Teilabschnitten werden die aufgetretenen Effizienzgewinne bzw. -verluste im einzelnen näher interpretiert.

\subsection{Die Änderung der Struktur der Konsumsteuersätze}

Auf den ersten Blick mag es überraschen, daß sich die mit der Einräumung eines allgemeinen Vorsteuerabzugsrechts - mit anderen Worten der konsequenten Ausgestaltung einer Umsatzsteuer vom Konsumtyp verbundene modifizierte Konsumbesteuerung vergleichsweise stark negativ auswirkt. Betrachtet man jedoch die in Tabelle 15 ausgewie- 
Tabelle 15: Die Veränderung der effektiven Konsumsteuersätze

\begin{tabular}{|c|c|c|}
\hline & \multicolumn{2}{|c|}{ Steuersätze (in \%) } \\
Gut & Simulation 1 & Simulation 2 \\
\hline 1 & 7.47 & 8.11 \\
2 & 13.36 & 14.75 \\
3 & 12.65 & 13.85 \\
4 & 14.38 & 15.76 \\
5 & 14.34 & 15.76 \\
6 & 13.93 & 15.31 \\
7 & 12.83 & 14.08 \\
8 & 14.36 & 15.76 \\
9 & 7.82 & 8.45 \\
10 & 14.32 & 15.76 \\
11 & 13.45 & 14.53 \\
12 & 10.76 & 11.71 \\
13 & 2.01 & 0.0 \\
14 & 2.71 & 0.0 \\
15 & 4.85 & 0.0 \\
\hline
\end{tabular}

senen, den Konsum im jeweiligen Gleichgewicht (mit-) bestimmenden Steuersätze, erscheint das Ergebnis weniger verblüffend.

Die in Tabelle 15 unter Simulation 1 aufgelisteten Steuersätze beziehen sich hierbei auf die im Ausgangsgleichgewicht bestehende effektive Besteuerung des Konsums. Die neuen Steuersätze gehen aus der zweiten Differentialinzidenz hervor. Ein Vergleich beider Steuersatzprofile zeigt zum einen, daß die neuen Konsumsteuersätze für die letzen drei Güterarten nun tatsächlich gleich Null sind, und zum anderen, daß die übrigen Steuersätze aufgrund des aus der Nullbesteuerung resultierenden Ausfalls an Steueraufkommen erhöht werden mußten. Dies erinnert an ein von HatTa [1986] entwickeltes Theorem. Er wies nach, daß sich die Wohlfahrt in einer Ein-Personen-Ökonomie unter bestimmten Umständen auch bei variablem Arbeitsangebot dann verbessert, wenn der niedrigste Konsumsteuersatz erhöht und zum Ausgleich der höchste Steuersatz gesenkt wird. Dieser Effekt scheint sich hier in umgekehr- 
ter Richtung zu realisieren. Während die niedrigsten ursprünglichen Steuersätze auf Null reduziert wurden, erhöhten sich gleichzeitig alle anderen.

Allerdings ist das Theorem nicht vorbehaltlos anwendbar. Insbesondere geht Hatta von konstanten Produzentenpreisen und einer geschlossenen Volkswirtschaft aus. Darüber hinaus werden von ihm keine weiteren verzerrenden Steuern berücksichtigt. In unserem Modell dürften hier vor allem die bestehenden Produktionssteuersätze Auswirkungen auf die allokativen Effekte einer Konsumsteuer ausüben. Zur Einschätzung der von beiden Steuerarten insgesamt ausgelösten Effekte ist es jedoch nicht möglich, die Produktionssteuersätze einfach zu den Konsumsteuersätzen hinzuzuaddieren, wie es simple Äquivalenzbeziehungen nahelegen. Diese Äquivalenz wird durch die Berücksichtigung von Vorleistungsverflechtungen sowie durch die Betrachtung einer offenen Volkswirtschaft zerstört.

Mit der Unterteilung der ursprünglichen Steuerreform in drei Stufen ist es nun zwar möglich, die simultane Veränderung der Besteuerung der verschiedenen Komponenten der Endnachfrage schrittweise durchzuführen, dennoch sind alle Steuern auch für die allokativen Effekte jeder einzelnen Maßnahme entscheidend. So hat beispielsweise die Veränderung der Konsumsteuer auch Auswirkung auf die internationale Wettbewerbsfähigkeit der Volkswirtschaft, ebenso wie die Produktionssteuersätze für die Effizienzwirkung einer bestimmten Struktur der Konsumsteuersätze verantwortlich sind. Wir werden auf diesen Punkt nochmals zurückkommen.

\subsection{Die Änderung der Besteuerung des Außenhandels}

Mit der dritten Differentialinzidenz wurden nun auch die von Umsatzsteuerbefreiungen ausgehenden indirekten Besteuerungseffekte auf den Außenhandel neutralisiert. Zur Erinnerung sei wiederholt, daß die Umsatzsteuer dabei einer Steuer auf Exporte und einer Subvention der Importe gleichkam. Da diese Steuer- bzw. Subventionssätze in der vorangehenden Simulation 2 nicht verändert wurden, können sie wieder aus Tabelle 4 in Kapitel II, Abschnitt (4) entnommen werden. Wie bereits erwähnt, sind die effektiven Sätze im neuen - dem mit Differentialinzidenz 3 errechneten - Gleichgewicht gleich Null. Die beiden mitt- 
leren Spalten von Tabelle 16 geben die von Differentialinzidenz 2 (= alt) zu Differentialinzidenz $3(=$ neu) eingetretenen Veränderungen der Netto-Exportmengen $\left(X_{i}-M_{i}\right)$ an. In der letzten Spalte sind die mit dem Wegfall der Außenhandelsbesteuerung verbundenen geänderten Konsumsteuersätze aufgelistet.

Tabelle 16: Veränderung der Netto-Exportmengen

\begin{tabular}{|c|c|c|c|}
\hline & \multicolumn{2}{|c|}{ Netto-Exporte } & Konsumsteuersätze \\
Gut & alt & \multicolumn{1}{c|}{ neu } & (in \%) \\
\hline 1 & -361.53 & -362.09 & 8.01 \\
2 & -669.59 & -670.09 & 14.57 \\
3 & -16.01 & -20.55 & 13.68 \\
4 & 605.66 & 601.62 & 15.57 \\
5 & 508.88 & 503.84 & 15.57 \\
6 & 147.93 & 142.97 & 15.12 \\
7 & -78.36 & -79.29 & 13.90 \\
8 & -197.27 & -198.23 & 15.57 \\
9 & -166.95 & -168.31 & 8.34 \\
10 & 323.60 & 320.68 & 15.57 \\
11 & 204.53 & 208.93 & 14.34 \\
12 & 353.35 & 351.38 & 11.56 \\
13 & -231.38 & -223.18 & 0.0 \\
14 & -422.01 & -419.10 & 0.0 \\
\hline
\end{tabular}

Bildet man Mengenindizes über die Entwicklung der gesamtwirtschaftlichen Netto-Exporte bzw. Netto-Importe, so ist zu beobachten, $\mathrm{da} B$ im neuen Gleichgewicht sowohl Exportmengen als auch Importmengen gesunken sind, wobei die Exportmengen vergleichsweise stärker absinken. Insgesamt geht damit eine leichte Verbesserung der Termsof-Trade von 0.998 in der alten Situation auf 1.002 im neuen Gleichgewicht einher ${ }^{125}$. Wir wollen versuchen, diese Entwicklung des Außen-

${ }^{125}$ Die Berechnung der Terms-of-Trade erfolgte durch das Verhältnis eines Laspeyres-Mengenindex für Importe zu einem Mengenindex gleichen Typs für Exporte. 
handels anhand einer Grafik zu veranschaulichen. Bedingt durch die Beschränktheit der graphischen Darstellung auf zwei Dimensionen ist es jedoch unmöglich die Entwicklung von 14 Güterströmen exakt (und simultan, wie es eigentlich notwendig wäre) nachzuvollziehen. Man kann die Effekte nur anhand zweier typisierter Güter plausibilisieren.

Abbildung 5: Wohlfahrtseffekte durch Abschaffung der impliziten Besteuerung des AuBenhandels

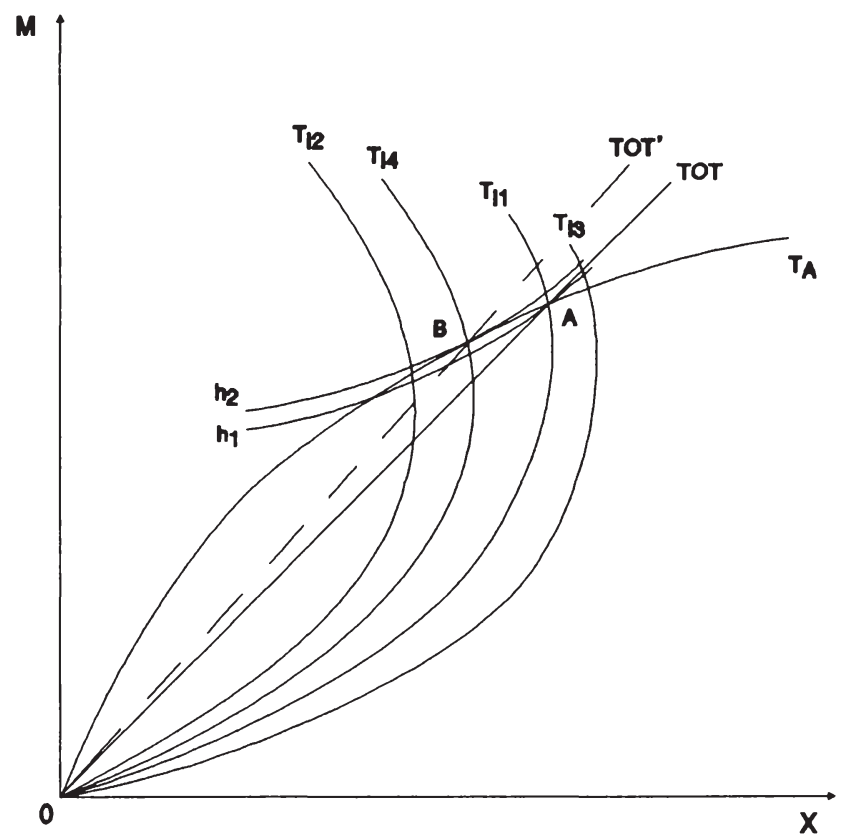

In Abbildung 5 ist das ursprüngliche Außenhandelsgleichgewicht durch den Schnittpunkt A der inländischen Tauschkurve $\mathrm{T}_{\mathrm{I} 1}$ mit der ausländischen Tauschkurve $\mathrm{T}_{\mathrm{A}}$ gekennzeichnet. Die durchgezogene Linie 0A symbolisiert hierbei die im Ausgangszustand geltenden Terms- 
of-Trade ${ }^{126}$. Aus der realen Theorie der Außenwirtschaft ist bekannt, daß eine Besteuerung der Exporte die inländische Tauschkurve nach links, eine Subvention der Importe die Tauschkurve hingegen nach rechts dreht ${ }^{127}$. Auf unsere Situation angewandt, werden sich folglich Drehungen in umgekehrter Richtung einstellen. Die Abschaffung der Importsubvention führt zu einer Drehung der ursprünglichen Tauschkurve nach links - beispielsweise nach $\mathrm{T}_{\mathrm{I} 2}$-, die Reduktion der Exportsteuersätze führt wiederum zu einer Drehung der inländischen Tauschkurve von $\mathrm{T}_{\mathrm{I} 2}$ nach rechts - bspw. auf $\mathrm{T}_{\mathrm{I} 3}$. Der daraus resultierende Gesamteffekt ist jedoch ambivalent, so daß $\mathrm{T}_{\mathrm{I} 3}$ auch links von $\mathrm{T}_{\mathrm{I} 1}$ liegen könnte.

Schließlich sind noch zwei weitere Effekte zu beachten, die aus der endogenen Angleichung der Konsumsteuersätze hervorgehen. Wie die letzte Spalte von Tabelle 16 zeigt, sind die Konsumsteuersätze im neuen Gleichgewicht niedriger als in der Ausgangssituation ${ }^{128}$. Offensichtlich wird durch die Abschaffung der Außenhandelsbesteuerung - insbesondere der Importsubvention - ein Steuermehraufkommen erzielt, das zu dieser allgemeinen Konsumsteuersenkung führt. Die dadurch hervorgerufene Steigerung der Konsumnachfrage bewirkt dabei eine tendenzielle Steigerung der inländischen Importnachfrage bei gleichzeitiger Senkung des Exportangebots. Allerdings sind die letzten beiden Güter 13 und 14 von dieser Tendenz nicht bzw. in nur sehr geringem Ausmaß betroffen, da die relevanten Konsumsteuersätze bereits im Ausgangsgleichgewicht gleich Null sind ${ }^{129}$.

${ }^{126}$ Explizit berechnen sich die Terms-of-Trade als Tangens des von der Geraden $O A$ und der $X$-Achse eingeschlossenen Winkels.

${ }^{127}$ Bei einem gegebenen (ungestörten) realen Tauschverhältnis bewirkt die Einführung einer Exportsteuer, daß die Inländer nun weniger Exporte als in der Freihandelssituation für eine bestimmte Importmenge anbieten, da ein Teil des ursprünglichen Exportvolumens als Steuer abzuführen ist. Eine Importsubvention läßt sich dahingehend beschreiben, daß zu einem bestimmten Tauschverhältnis das Inland nun bereit ist, die gleiche Exportmenge für weniger Importe als in der Freihandelssituation anzubieten, da der Staat die entstandene Lücke durch Subventionen ausgleicht (vgl. hierzu auch BHAGWATI/ SRINIVASAN [1983], S. 108 ff.).

${ }^{128}$ Die entsprechenden Steuersätze der Ausgangssituation können der letzten Spalte von Tabelle 15 entnommen werden.

129 Dies gilt insbesondere für unsere unterstellten Cobb-Douglas-Nachfragefunk- 
Diese abermalige Drehung der inländischen Tauschkurve nach links wird verstärkt durch die mit der Senkung der Konsumsteuersätze einhergehende Änderung der relativen Konsumentenpreise. In der dargestellten Zwei-Güter-Ökonomie würde die mit der Einführung einer partiellen Konsumsteuer auf das Netto-Exportgut verbundene Erhöhung des entsprechenden Konsumentenpreises allgemein eine Drehung der Tauschkurve nach rechts verursachen (siehe auch GEORgAKopouLOS [1974]). Berücksichtigt man nun, daß die von der Konsumsteuersenkung nicht betroffenen Güter 13 und 14 Netto-Importgüter sind, wird eine Senkung der Konsumsteuersätze Netto-Exportgüter vergleichsweise stärker treffen als Netto-Importgüter, woraus eine weitere Drehung der Tauschkurve nach links - z. B. nach $\mathrm{T}_{I 4}$ - resultiert.

Im Schnittpunkt B der aus dieser sukzessiven Betrachtung der einzelnen Effekte letztendlich hervorgehenden neuen inländischen Tauschkurve $\mathrm{T}_{\text {I4 }}$ mit ihrem ausländischen Gegenstück $\mathrm{T}_{\mathrm{A}}$ ist schließlich das neue Außenhandelsgleichgewicht erreicht. Die durch die gestrichelte Linie 0B gekennzeichneten Terms-of-Trade TOT ${ }^{\prime}$ haben sich verbessert. Ebenso realisiert das Inland in B einen Punkt auf einer höheren Handels-Indifferenzkurve $h_{2}$ und damit eine höhere Wohlfahrt.

Die mit der graphischen Darstellung anhand von zwei Gütern typisierte Entwicklung der Warenströme kommt grundsätzlich auch in Tabelle 16 zum Ausdruck. Letztere muß jedoch etwas differenzierter betrachtet werden. $\mathrm{Da}$ auch die Konsumsteuersätze für NettoImportgüter - bspw. Gut 1-3 - sinken, ist eine Erhöhung der entsprechenden Importmengen feststellbar. Diese Entwicklung steht nicht im Gegensatz zu unserem Erklärungsmuster, sie trägt vielmehr dazu bei, daß die Exportmengen, gesamtwirtschaftlich betrachtet, stärker sinken als die Importmengen. In der Zunahme der Exportmenge des Gutes 11 von 204.53 auf 208.93 Einheiten kommt dagegen zum Ausdruck, daß die Abschaffung des höchsten Exportsteuersatzes alle nachgelagerten Effekte überwiegt.

tionen, die eine Kreuzpreis-Elastizität von Null aufweisen. Eine verhältnismäBig geringe Steigerung der Güternachfrage entsteht dennoch durch die Senkung des Preisindex für Konsumgüter $p$ in Relation zum Lohn, womit ein Substitutionseffekt zugunsten der gesamten Konsumgüternachfrage ausgelöst wird und auch die Güter 13 und 14 mit einschließt. 


\subsection{Die Änderung der Investitionsteuersätze}

Mit der Aufhebung der bisher unverändert gebliebenen effektiven Investitionsteuersätze - Differentialinzidenz 4 - ist der Übergang von Umsatzsteuerbefreiung zu allgemeinen Nullsteuersätzen schließlich vollzogen. Auch hier ergibt sich ein auf den ersten Blick überraschendes Resultat. Die Abschaffung der Investitionsteuer und somit der davon ausgehenden allokativen Verzerrung bewirkt eine Wohlfahrtssenkung in Bezug auf das mit der vorangehenden Simulation 3 erreichte Gleichgewicht.

Der Wert der Äquivalenten Variation ist mit -50 Mill. DM zwar vergleichsweise gering. Auf der anderen Seite muß jedoch berücksichtigt werden, daß von der zum Ausgleich des staatlichen Budgets vorgenommenen allgemeinen Senkung der Konsumsteuer wohl ein positiver Wohlfahrtseffekt ausgeht, der die negative Wirkung der Investitionsteuersenkung nicht kompensieren kann. Wie ist dieses Ergebnis zu interpretieren?

Die Besteuerung der Investitionen verursacht eine Änderung des Kapitaleinsatzes im Unternehmensbereich. Entscheidend für die Kapitalnachfrage (pro Einheit Output) der Unternehmen sind jedoch die gesamten Kapitalnutzungskosten $\delta_{i}\left(1+\tau_{i}^{I}\right) q^{I}+\left(1+\tau_{i}^{k}\right) r$ im Vergleich zum Preis für Arbeit. Je einheitlicher die Kapitalnutzungskosten sind, desto geringer sind auch die Verzerrungen der Faktorallokation. Neben den Investitionsteuern $\tau_{i}^{I}$ sind deshalb u. a. auch die Produzentenpreise und vor allem die Kapitalsteuersätze maßgebend für einen effizienten Faktoreinsatz. Tabelle 17 zeigt die von Differentialinzidenz 3 (= alt) zu Differentialinzidenz 4 (= neu) eingetretene Änderung dieser Kapitalnutzungskosten.

Erinnern wir uns, daß im Benchmark Equilibrium nur zwei unterschiedliche effektive Investitionsteuersätze existieren: ein Steuersatz von $0.33 \mathrm{v}$. H. für vorsteuerabzugsberechtigte Unternehmen und ein Steuersatz von 13.99 v. H. für nichtabzugsberechtigte. Vor diesem Hintergrund zeigt Tabelle 17, daß die Kapitalnutzungskosten für die Sektoren $1, \ldots, 12$ tendenziell ansteigen, während die für die Sektoren 13,14 und 15 relevanten tendenziell sinken ${ }^{130}$. Zieht man die Varianz als In-

${ }^{130}$ Der Anstieg der Kapitalnutzungskosten in den ersten zwölf Unternehmen 
Tabelle 17: Die Veränderung der Kapitalnutzungskosten

\begin{tabular}{|c|c|c|}
\hline & \multicolumn{2}{|c|}{ Kapitalnutzungskosten } \\
Sektor & alt & neu \\
\hline 1 & 1.0829 & 1.0842 \\
2 & 1.2400 & 1.2415 \\
3 & 1.5681 & 1.5701 \\
4 & 1.1547 & 1.1561 \\
5 & 1.2694 & 1.2708 \\
6 & 1.1612 & 1.1627 \\
7 & 1.2012 & 1.2027 \\
8 & 1.2222 & 1.2236 \\
9 & 1.4749 & 1.4768 \\
10 & 1.1328 & 1.1343 \\
11 & 1.1222 & 1.1236 \\
12 & 1.1451 & 1.1466 \\
13 & 1.0781 & 1.0706 \\
14 & 1.1628 & 1.1595 \\
15 & 1.0346 & 1.0324 \\
\hline
\end{tabular}

dikator für die Streuung der Kapitalnutzungskosten heran, ergibt sich für die Ausgangssituation ein Wert von 0.0208, im neuen Gleichgewicht ein Wert von 0.0212 . Von den Investitionsteuersätzen ging somit eine verzerrungshemmende Wirkung auf die Faktorallokation aus, die im neuen Gleichgewicht entfällt.

Diese Erläuterung der Effizienzwirkung unserer effektiven Investitionsteuer ist, streng genommen, nicht ganz vollständig. Analog zu den Kapitalsteuern verursacht eine Besteuerung der Investitionen nicht nur die beschriebenen Faktorsubstitutionseffekte, sondern aufgrund der steuerbedingten Erhöhung der Produzentenpreise auch Output-

erklärt sich dadurch, daß die Aufhebung der Investitionsteuer zu einer generellen Steigerung der Kapitalnachfrage und damit zu einer Erhöhung des Mietpreises $r$ für Kapital führt. Diese Preissteigerung kann mit dem Wegfallen der relativ geringen Investitionsteuersätze $\tau_{i}^{I}(i=1, \ldots, 12)$ nicht kompensiert werden. 
effekte $^{131}$. Letztere werden darüber hinaus auch von der bestehenden Struktur der Produktionssteuersätze ausgelöst. Man müßte folglich die von allen im Unternehmensbereich erhobenen Steuern verursachten Outputeffekte mit denjenigen vergleichen, die ohne die Investitionsteuern entstehen würden. Da sich diese Effekte über die Produktionsverflechtung durch das gesamte ökonomische System ziehen, und darüber hinaus via Investitionsgüterpreise wiederum auf die Wertschöpfung rückwirken, war es nicht möglich, die allokative Wirkung der Outputeffekte zu beschreiben. So begnügen wir uns mit der Feststellung, daß die mit dem Wegfallen der Investitionsteuersätze verbundenen Outputeffekte vergleichsweise gering sind, zumal bereits die Kapitalnutzungskosten nur einen relativ kleinen Teil der Produzentenpreise ausmachen.

Damit sind die wesentlichen Effizienzwirkungen, die durch den aufkommensneutralen Übergang von Umsatzsteuerbefreiungen zu Nullsteuersätzen auftreten, erklärt. Zusammenfassend können wir feststellen, daß aus den überaus vielschichtigen allokativen Effekten, insgesamt gesehen, ein relativ kleiner Wohlfahrtsgewinn resultiert, der hauptsächlich auf einer Verbesserung des Weltmarkttauschverhältnisses zugunsten des Inlands basiert.

Dagegen führt die mit der Einräumung eines allgemeinen Vorsteuerabzugsrechts einhergehende Änderung der effektiven Konsumbesteuerung zu einem Wohlfahrtsverlust. Er ist hauptsächlich durch die notwendige Anhebung aller Steuersätze ungleich Null bedingt, da der Nachholeffekt von unechten Befreiungen im neuen Umsatzsteuersystem entfällt. Auf diese Konsequenz wurde bereits im letzten Abschnitt von Kapitel I hingewiesen und durch die Simulationsrechnung nun bestätigt. Angesichts dieser Wirkung denkt man unmittelbar an die nahezu ausnahmslos als beste Version einer Umsatzsteuer betrachtete allgemeine Umsatzsteuer mit einem für alle Güter bzw. Sektoren einheitlichen Steuersatz.

In der Tat wäre auch hinsichtlich der unterstellten funktionalen Form der Nutzenfunktion - die CES/Cobb-Douglas Nutzenfunktion ist homothetisch und streng separabel zwischen Güterkonsum und Freizeit -

${ }^{131}$ Siehe hierzu bspw. HARBERgER [1962] oder MCLURE [1975]. 
ein einheitlicher Umsatzsteuersatz optimal ${ }^{132}$, wenn keine weiteren allokativen Verzerrungen bestünden. Im vorliegenden komplexeren Umfeld müssen jedoch auch die Produktions- und die Kapitalsteuer in derartige Effizienzerwägungen miteinbezogen werden. Aus diesem Grund bestimmen wir im nächsten Abschnitt zuerst die von der Produktionssteuer ausgehende effektive Besteuerung der einzelnen Komponenten der Endnachfrage und wenden uns im Anschluß daran einer zweiten Steuerreform, nämlich der Einführung einer allgemeinen Umsatzsteuer, zu.

\section{Die Erweiterung der effektiven Steuersätze}

Das Konzept der effektiven Steuersätze wurde bisher dazu benutzt, die tatsächliche Steuerwirkung eines Umsatzsteuersystems zu ermitteln. Wir gingen dabei von den jeweiligen Budgetrestriktionen aus und berechneten die efffektiven Steuersätze gerade so, daß sich bei einer äquivalenten Besteuerung von Konsum, Investitionen und Außenhandel dieselben Rahmenbedingungen für die Wirtschaftssubjekte ergaben, wie bei der ursprünglichen Besteuerung der Umsätze. Diese Vorgehensweise wird nun auch auf die Produktionssteuern angewendet. Wir bestimmen die zu einem gegebenen System von Umsatz- und Produktionssteuern äquivalente Besteuerung der drei Endnachfragekategorien - wiederum unter der Bedingung, daß beide Steuersysteme die gleiche Güter- und Faktorallokation und somit dasselbe Gleichgewicht hervorbringen. Da die Berechnung der effektiven Steuersätze ganz analog zu der in Kapitel II präsentierten Methode abläuft, können wir uns hier mit einer kurzen Skizzierung der Vorgehensweise begnügen.

Ausgangspunkt sind bekanntlich die Null-Profit-Bedingungen der Unternehmen. Bei Erhebung der Umsatz- und der Produktionssteuern können sie durch

$$
q_{i} Q_{i}=\sum_{j=1}^{n} a_{j i} q_{j} Q_{i}+\tau_{i}^{q} q_{i} Q_{i}+q^{I} \delta_{i} K_{i}+\left(1+\tau_{i}^{k}\right) r K_{i}+w L_{i}
$$

${ }^{132}$ Siehe u. a. Deaton $[1977,1979]$ oder AtKinson/Stiglitz [1980]. 


$$
\begin{array}{r}
q_{h} Q_{h}=\sum_{j=1}^{n} a_{j h}\left(1+\tau_{j}^{u}\right) q_{j} Q_{h}+\tau_{h}^{q} q_{h} Q_{h}+p^{I} \delta_{h} K_{h}+ \\
+\left(1+\tau_{h}^{k}\right) r K_{h}+w L_{h}
\end{array}
$$

beschrieben werden, wobei die obere Gleichung für die im Benchmark Gleichgewicht steuerpflichtigen Unternehmen $i=1, \ldots, 12$, die untere für die zunächst umsatzsteuerbefreiten Unternehmen $h=13,14,15$ relevant ist. Nach Division durch $Q_{i}$ bzw. $Q_{h}$ erhalten wir daraus in Matrixschreibweise

$$
q=\left(A^{\prime}+A^{\prime}{ }_{m} \hat{\tau}^{u}+\hat{\tau}^{q}+B^{\prime}+B^{\prime}{ }_{m} \hat{\tau}^{u}\right) q+W_{e}
$$

bzw.

$$
\left(I-A^{\prime}-A^{\prime}{ }_{m} \hat{\tau}^{u}-\hat{\tau}^{q}-B^{\prime}-B^{\prime}{ }_{m} \hat{\tau}^{u}\right) q=W_{e},
$$

mit $A^{\prime}$ als der transponierten Input-Koeffizientenmatrix, $B^{\prime}$ der transponierten Matrix der Reinvestitionsgüter-Anteile $\left([B]_{i j}=\beta_{j} \delta_{i} k_{i}\right)$ und $\hat{\tau}^{q}, \hat{\tau}^{u}$ als jeweilige Diagonalmatrix der Produktion- bzw. Umsatzsteuersätze. Die Matrizen $A^{\prime}{ }_{m}$ und $B^{\prime}{ }_{m}$ weisen wiederum in den für umsatzsteuerbefreite Unternehmen relevanten Zeilen 13,14 und 15 dieselben Elemente auf wie $A^{\prime}$ bzw. $B^{\prime}$, alle anderen Elemente sind gleich Null. Eine typische Zeile - bspw. Zeile $i$ - des Spaltenvektors $W_{e}$ besteht schließlich aus $\left(1+\tau_{i}^{k}\right) r k_{i}+w l_{i}$.

Stellt man dieses Gleichungssystem zur Bestimmung der Produzentenpreise bei Erhebung einer Umsatz- und einer Produktionssteuer dem hierzu entsprechenden Preisbildungssystem bei einer äquivalenten Besteuerung der Endnachfragekomponenten

$$
\left(I-A^{\prime}-B^{\prime}\right) \tilde{q}=W_{e}
$$

gegenüber, kann durch die Differenz $t:=q-\tilde{q}$ der jeweils geltenden Produzentenpreise zunächst der implizite Steuerbetrag pro Besteuerungseinheit

$$
t=\left(I-A^{\prime}-B^{\prime}\right)^{-1}\left[\left(A_{m}^{\prime}+B_{m}^{\prime}\right) \hat{\tau}^{u}+\hat{\tau}^{q}\right] q
$$


berechnet werden. Danach sind in gewohnter Vorgehensweise die effektiven Steuersätze über

$$
\begin{array}{rlrl}
\tilde{\tau}_{i} & =\frac{t_{i}}{\tilde{q}_{i}} & i=1, \ldots, 15 \\
\tau_{i}^{c}=\tau_{i}^{u}+\tau_{i}^{u} \tilde{\tau}_{i}+\tilde{\tau}_{i} & i=1, \ldots, 15 \\
\tau_{i}^{X}=\tilde{\tau}_{i} & i=1, \ldots, 14 \\
\tau_{i}^{M}=\frac{\tilde{\tau}_{i}}{1+\tilde{\tau}_{i}} & i=1, \ldots, 14 \\
\tau_{i}^{I}=\frac{\sum_{j} \beta_{j} q_{j}}{\sum_{j} \beta_{j} \tilde{q}_{j}} & i=1, \ldots, 12 \\
\tau_{i}^{I}=\frac{\sum_{j} \beta_{j} \tau_{j}^{u} q_{j}}{\sum_{j} \beta_{j} \tilde{q}_{j}} & i=13,14,15
\end{array}
$$

bestimmbar.

Noch bessere Beurteilungsmöglichkeiten der insgesamt erhobenen Steuern ergäben sich, wenn auch die Kapitalsteuern in die effektiven Steuersätze aufnehmbar wären. Es zeigt sich jedoch, daß dieses Vorgehen nicht auf die Kapitalsteuern erweitert werden kann. Folgende Plausibilitätsüberlegung soll dies verdeutlichen. Grundsätzlich beeinflußt eine nach Sektoren differenzierte Kapitalsteuer die relativen BruttoFaktorpreise und damit das Faktoreinsatzverhältnis sowie die relativen Produzentenpreise und damit die Güternachfrage bzw. -produktion. Würde man nun anstelle der Kapitalsteuersätze einen impliziten Steuersatz auf den Produzentenpreis erheben, so wird der Faktoreinsatz nicht direkt von der Steuer verzerrt. Der implizite Steuersatz führt somit nicht zu einem in beiden Situationen identischen Faktoreinsatzverhältnis. Aus diesem Grund können spezifische Faktorsteuern im Rahmen unseres Modells nicht durch eine äquivalente Besteuerung der Endnachfragekategorien ersetzt werden. 
Immerhin erlauben die erweiterten effektiven Steuersätze nun die Beurteilung der tatsächlichen Besteuerungswirkungen, die von Umsatzund Produktionssteuern gemeinsam ausgehen. Wir wollen mit dieser neuen Erkenntnis die ursprüngliche Steuerreform - die Einführung von Nullsteuersätzen - nochmals kurz beleuchten. In Tabelle 18 sind die erweiterten effektiven Steuersätze des Benchmark Equilibriums den bei allgemeinem Vorsteuerabzugsrecht geltenden gagenübergestellt. Auf den Ausweis der Importsubventionssätze wurde hierbei verzichtet, da sie implizit mit den Exportsteuersätzen gegeben sind.

Tabelle 18: Die Veränderung der erweiterten effektiven Steuersätze

\begin{tabular}{|c|rrr|rrr|}
\hline & \multicolumn{3}{|c|}{ Benchmark Equilibrium } & \multicolumn{3}{c|}{ Counterfactual Equilibrium } \\
Gektor & \multicolumn{1}{|c}{$\tau_{i}^{c}$} & \multicolumn{1}{c|}{$\tau_{i}^{X}$} & \multicolumn{1}{c|}{$\tau_{i}^{I}$} & $\tau_{i}^{c}$ & $\tau_{i}^{X}$ & $\tau_{i}^{I}$ \\
\hline 1 & 6.32 & -0.82 & 1.72 & 06.92 & -1.07 & 1.39 \\
2 & 16.24 & 2.77 & 1.72 & 17.61 & 2.54 & 1.39 \\
3 & 20.00 & 6.86 & 1.72 & 21.22 & 6.52 & 1.39 \\
4 & 15.85 & 1.62 & 1.72 & 17.19 & 1.28 & 1.39 \\
5 & 16.01 & 1.76 & 1.72 & 17.40 & 1.45 & 1.39 \\
6 & 15.49 & 1.67 & 1.72 & 16.84 & 1.37 & 1.39 \\
7 & 14.48 & 1.76 & 1.72 & 15.69 & 1.46 & 1.39 \\
8 & 16.04 & 1.79 & 1.72 & 17.41 & 1.47 & 1.39 \\
9 & 16.03 & 7.93 & 1.72 & 16.67 & 7.61 & 1.39 \\
10 & 16.11 & 1.85 & 1.72 & 17.53 & 1.57 & 1.39 \\
11 & 12.81 & -0.08 & 1.72 & 13.83 & -0.56 & 1.39 \\
12 & 12.78 & 2.15 & 1.72 & 13.70 & 1.82 & 1.39 \\
13 & 2.62 & 2.62 & 15.58 & 00.60 & 0.60 & 1.39 \\
14 & 4.99 & 4.99 & 15.58 & 02.22 & 2.22 & 1.39 \\
15 & 6.07 & & 15.58 & 01.16 & & 1.39 \\
\hline
\end{tabular}

Betrachten wir zunächst die effektiven Steuersätze im Ausgangsgleichgewicht. Durch die Einbeziehung der Produktionssteuersätze zeigen sich doch relativ große Abweichungen in der tatsächlichen Besteue- 
rung des Konsums gegenüber den nominellen Umsatzsteuersätzen. Beispielsweise kommt mit dem für Gut 3 relevanten Steuersatz von 20 v. H. zum Ausdruck, daß der Konsum chemischer Erzeugnisse am höchsten steuerlich belastet ist. Ebenso zeigt der effektive Steuersatz für Nahrungsmittel und Getränke (Sektor 9), daß die tatsächliche Besteuerung mehr als doppelt so hoch ist, wie es der nominale Steuersatz angibt. Diese Wirkung ist offensichtlich zum Teil auf eine Reihe spezieller Verbrauchsteuern zurückzuführen, die in dem Produktionssteuersatz zusammengefaßt sind. Nebenbei bemerkt, erhält unter diesem Gesichtspunkt auch das Argument für eine Staffelung der Umsatzsteuersätze aufgrund der regressiven Wirkung der Steuer ein anderes Gewicht.

Selbst die nominell befreiten Leistungen der Versicherungen und des Wohnungsvermietungssektors (Sektor 14) werden mit einer Steuer von immerhin rund 5 Prozent belastet. Dagegen führt die Subvention der Land- und Forstwirtschaft (Sektor 1) und des Transportwesens (Sektor 11) zu einer etwas geringeren tatsächlichen Belastung, als es die nominellen Steuersätze angeben. Bemerkenswert ist noch, daß die effektiven Investitionsteuersätze für umsatzsteuerbefreite Unternehmen rund 15.6 v. H. betragen, falls man die Wirkung der Produktionssteuern in die Betrachtung miteinbezieht.

Vergleichen wir die effektiven Steuersätze des Ausgangsgleichgewichts nun mit denjenigen des Counterfactual Equilibrium so ist zwar festzustellen, daß Außenhandel- und Investitionsteuersätze nicht gänzlich entfallen, die in den vorangehenden Abschnitten geschilderte tendenzielle Entwicklung bleibt jedoch erhalten. Die effektiven Konsumsteuersätze fallen weiter auseinander, während die Außenhandel- und Investitionsteuersätze absinken bzw. vereinheitlicht werden.

Lediglich die Exportsteuersätze für das erste und das elfte Gut zeigen einen anderen Verlauf. Durch die Berücksichtigung der Produktionssteuer wird deutlich, daß der Export beider Güter bereits im Ausgangsgleichgewicht effektiv subventioniert wird (und die Importe besteuert werden). Der Übergang zu Nullsteuersätzen bewirkt nun eine Zunahme dieser Subvention im neuen Gleichgewicht. Da eine Erhöhung der Subvention dieselbe Wirkung auf die inländische Tauschkurve zeitigt wie eine Senkung der Steuer, ändert sich nichts an der ökonomischen Erklärung der aufgetretenen Wohlfahrtseffekte. 


\section{Einheitliche und optimale Umsatzsteuersätze}

Aus zahlreichen, sehr verschiedenen Gründen wird von den meisten Befürwortern einer Umsatzsteuer die Anwendung eines einheitlichen Steuersatzes auf alle Güter als beste Erhebungsform für diese Steuer angesehen, wobei auch Effizienzüberlegungen ins Felde geführt werden ${ }^{133}$. Mit den nachfolgenden Steuerreformmaßnahmen wollen wir deshalb die Effizienzwirkungen einer allgemeinen Umsatzsteuer im Rahmen unseres Systems untersuchen.

Nach den im vorangehenden Abschnitt dargestellten Ergebnissen ist jedoch auch ohne Simulationsrechnung zu vermuten, daß eine allgemeine Umsatzsteuer unter allokativen Gesichtspunkten nicht unbedingt die beste Variante der Steuer sein muß. Aus diesem Grund stellt sich vornehmlich die Frage, ob bzw. inwieweit eine Verbesserung der Wohlfahrt durch Angleichung der Umsatzsteuersätze erreicht werden kann.

Zunächst gehen wir davon aus, daß aus den in Kapitel II, Abschnitt (4) genannten erhebungstechnischen Gründen nur die für Unternehmen $1, \ldots, 12$ maßgeblichen Umsatzsteuersätze aufkommensneutral vereinheitlicht werden können - der allgemeine Steuersatz beträgt in diesem Fall 11.94 v. H. -, während die Unternehmen 13,14 und 15 weiterhin steuerbefreit und nicht vorsteuer abzugsberechtigt sind. Diese Maßnahme erbringt eine Wohlfahrtssenkung von -142 Mill. DM gegenüber dem Ausgangsgleichgewicht. Würde man dagegen das Vorsteuer abzugsverbot für die umsatzsteuerbefreiten Unternehmen aufheben - der allgemeine Steuersatz steigt dann auf 13.38 Prozent an -, so ist mit $E V=37$ Mill. DM eine leichte Verbesserung der Wohlfahrt festzustellen.

Offensichtlich ist mit beiden partiellen Angleichungen der Umsatzsteuersätze keine deutlich spürbare Verbesserung der Wohlfahrtsposition der Volkswirtschaft zu erreichen. Unter Beibehaltung der Umsatzsteuerbefreiungen treten sogar leichte Wohlfahrtsverluste auf, die mit der Aufhebung des Vorsteuerabzugsverbots aus den in Abschnitt (2) geschilderten Gründen kompensiert werden können.

Betrachten wir die in Tabelle 19 für die jeweiligen Gleichgewichte

${ }^{133}$ Als repräsentative Beispiele hierfür sei auf MCLURE/TURE ([1972], S. 2) oder TAIT ([1988], S. 42 ff.) verwiesen. 
ausgewiesenen effektiven Steuersätze ${ }^{134}$, ist zu erkennen, daß die Vereinheitlichung der Umsatzsteuersätze nicht zu einer wesentlichen Angleichung der effektiven Konsumsteuersätze führt. Im Vergleich zum Benchmark Equilibrium - Tabelle 18 - ergeben sich mit der ersten Steuerreformmaßnahme nun zwei effektive Konsumsteuersätze von rund 20 v. H., während die für die letzten drei Sektoren maßgeblichen Niedrigsteuersätze nahezu unverändert bleiben. Auch die effektiven Exportund Investitionsteuersätze zeigen keine starke Veränderung gegenüber dem Ausgangsgleichgewicht. Dementsprechend ist die Wohlfahrtssenkung im wesentlichen auf die negative Entwicklung der effektiven Konsumsteuersätze zurückzuführen.

Eine sukzessive Betrachtung, im Stil der in Abschnitt (2) durchgeführten aufeinanderfolgenden Veränderung der effektiven Steuersätze, bestätigt diese Vermutung. Die Änderung der effektiven Konsumsteuersätze - unter Beibehaltung der im Ausgangsgleichgewicht bestehenden Export- und Investitionsteuersätze - erbringt bereits einen in Einkommensäquivalenten gemessenen Nutzenverlust von -121 Mill. DM. Dieser Nutzenverlust wird schließlich durch die anschließend geänderte effektive Besteuerung des Außenhandels $(E V=-140)$ und der Investitionen $(E V=-142)$ schwach verstärkt ${ }^{135}$. Dagegen erklärt sich die nahezu unveränderte Wohlfahrtsposition des mit der zweiten Steuerreform erreichten Gleichgewichts gegenüber dem Benchmark Equilibrium dadurch, daß die negative Effizienzwirkung der Konsumbesteuerung durch eine Verbesserung der Terms-of-Trade ausgeglichen wird (vgl. Teilabschnitt (2.3)). Damit kommt auch - nebenbei bemerkt - in diesen beiden Simulationen eine hinsichtlich der Güter- und Faktorallokation tendenziell negative Wirkung des Vorsteuerabzugsverbots zum Ausdruck.

Wäre es hingegen möglich, alle Umsatzsteuersätze zu vereinheitlichen, kann die Wohlfahrtsposition der Volkswirtschaft um einiges gesteigert werden, was die mit dieser Maßnahme erzielte positive Äqui-

134 Auch auf die Gefahr hin, Verwechslungen Vorschub zu leisten, werden im weiteren Verlauf dieses Abschnitts äquivalente Steuersätze, die aus der Zusammenfassung von Umsatz- und Produktionssteuern hervorgehen, als effektive Steuersätze bezeichnet.

${ }^{135}$ Für EV-Werte bildet das Benchmark Gleichgewicht den Referenzmaßstab. 
Tabelle 19: Effektive Steuersätze bei partieller Vereinheitlichung der Umsatzsteuer

\begin{tabular}{|c|rrr|rrr|}
\hline \multirow{2}{*}{ Gut/ } & \multicolumn{3}{|c|}{ Steuerreform 1} & \multicolumn{3}{c|}{ Steuerreform } \\
Sektor & \multicolumn{1}{|c|}{$\tau_{i}^{c}$} & \multicolumn{1}{c|}{$\tau_{i}^{X}$} & $\tau_{i}^{I}$ & \multicolumn{1}{c|}{$\tau_{i}^{c}$} & \multicolumn{1}{c|}{$\tau_{i}^{X}$} & $\tau_{i}^{I}$ \\
\hline 1 & 11.02 & -0.82 & 1.72 & 12.17 & -1.07 & 1.39 \\
2 & 15.05 & 2.77 & 1.72 & 16.26 & 2.54 & 1.39 \\
3 & 19.62 & 6.86 & 1.72 & 20.77 & 6.52 & 1.39 \\
4 & 13.76 & 1.62 & 1.72 & 14.83 & 1.28 & 1.39 \\
5 & 13.91 & 1.76 & 1.72 & 15.03 & 1.46 & 1.39 \\
6 & 13.81 & 1.67 & 1.72 & 14.94 & 1.37 & 1.39 \\
7 & 13.91 & 1.76 & 1.72 & 15.04 & 1.46 & 1.39 \\
8 & 13.95 & 1.79 & 1.72 & 15.04 & 1.47 & 1.39 \\
9 & 20.82 & 7.93 & 1.72 & 22.01 & 7.61 & 1.39 \\
10 & 14.02 & 1.85 & 1.72 & 15.16 & 1.57 & 1.39 \\
11 & 11.85 & -0.08 & 1.72 & 12.74 & -0.56 & 1.39 \\
12 & 14.35 & 2.15 & 1.72 & 15.44 & 1.82 & 1.39 \\
13 & 2.73 & 2.73 & 13.75 & 00.60 & 0.60 & 1.39 \\
14 & 4.97 & 4.97 & 13.75 & 02.22 & 2.22 & 1.39 \\
15 & 5.98 & & 13.75 & 01.16 & & 1.39 \\
\hline
\end{tabular}

Steuerreform 1: Einheitlicher Umsatzsteuersatz für Unternehmen 1-12, Unternehmen 13,14,15 umsatzsteuerbefreit und nicht vorsteuerabzugsberechtigt

Steuerreform 2: Einheitlicher Umsatzsteuersatz für Unternehmen 1-12, Unternehmen $13,14,15$ umsatzsteuerbefreit und vorsteuerabzugsberechtigt

valente Variation von 2693 Mill. DM deutlich macht ${ }^{136}$. Insbeson-

136 Tatsächlich werden nur die Steuersätze der Sektoren $1, \ldots, 14$ angeglichen. Da Sektor 15 nur intermediäre Güter sowie die konstant gehaltene Menge des Kollektivgutes produziert, zeitigt die umsatzsteuerliche Behandlung dieses Sektors keinerlei allokative Wirkungen, falls alle Unternehmen vorsteuerabzugsberechtigt sind. Eine Steuer (in beliebiger Höhe) auf den Umsatz von Sektor 15 erhoben, kann folglich von nachgelagerten Unternehmen als Vorsteuer geltend gemacht werden, während der Staat die auf seine Nachfrage anfallende Steuer gewissermaßen an sich selbst zahlt. 
dere erweisen sich bei einem schrittweisen Übergang zu der im neuen Gleichgewicht entstandenen Struktur der effektiven Steuersätze die Effizienzwirkungen, die durch die Änderung der effektiven Konsumsteuer $(E V=1380)$ verursacht wurden und die Effizienzwirkungen, die aus der darauf folgenden Veränderung der Außenhandelsbesteuerung ( $E V=2651$ ) hervorgingen, als nahezu gleich stark. Die Modifizierung der Investitionssteuer trägt dagegen nur relativ wenig zur insgesamt erzielten Verbesserung bei.

Wie aus der ersten Spalte von Tabelle $20 \mathrm{zu}$ entnehmen ist, zeigen die effektiven Konsumsteuersätze nun ein wesentlich einheitlicheres Bild als zuvor. Dadurch verringern sich nicht nur die Verzerrungen im Konsumbereich, sondern es verbessert sich auch das Weltmarkttauschverhältnis zugunsten des Inlands. Im Ausgangsgleichgewicht waren tendenziell diejenigen Güterarten mit einem relativ hohen effektiven Konsumsteuersatz behaftet, die gleichzeitig (netto) exportiert wurden. Die Veränderung der effektiven Konsumsteuersätze - unter Beibehaltung der im Ausgangsgleichgewicht gegebenen effektiven Außenhandelund Investitionsteuersätze - bewirkt deshalb eine Erhöhung der Termsof-Trade auf 1.004. Daß mit dem Wegfallen, der von den urspünglichen Umsatzsteuerbefreiungen hervorgerufenen impliziten Besteuerung des Außenhandels eine weitere Verbesserung der Terms-of-Trade verbunden ist, wurde bereits mehrfach erwähnt. Die im Vergleich zu den vorangehenden Simulationen deutlichere Erhöhung - die Terms-of-Trade steigen auf 1.007 - ist auf die bessere Ausgangssituation (bedingt durch die Vereinheitlichung der effektiven Konsumsteuersätze) zurückzuführen.

Der relativ große Einfluß, den die Entwicklung des Außenhandels auf die Wohlfahrt ausübt, kommt auch in der folgenden Simulation Steuerreform 4 - zum Vorschein: Würde man einen allgemeinen Umsatzsteuersatz in Höhe von 7.3 v. H. gepaart mit einen erhöhten Steuersatz von 14.7 v. H. für die typischen Netto-Importgüterindustrien 1,2,13 und 14 erheben, so erzielt man einen Wohlfahrtszuwachs von 2695 Mill. DM. Er ist damit nahezu mit dem durch Erhebung eines für alle Sektoren einheitlichen Umsatzsteuersatzes erreichten Wohlfahrtszuwachs identisch, obwohl die Struktur der effektiven Konsumsteuersätze nun stärkere Diskrepanzen aufweist ${ }^{137}$. Aus der schrittweisen Reform an-

${ }^{137}$ Auf eine explizite Angabe der effektiven Steuersätze wird verzichtet. 
Tabelle 20: Effektive Steuersätze bei vereinheitlichter Umsatzsteuer

\begin{tabular}{|c|rrc|rrc|r|}
\hline \multirow{2}{*}{ Gut/ } & \multicolumn{3}{|c|}{ Steuerreform 3} & \multicolumn{3}{|c|}{ Steuerreform 5} & \\
Sektor & \multicolumn{1}{|c|}{$\tau_{i}^{c}$} & \multicolumn{1}{c|}{$\tau_{i}^{X}$} & $\tau_{i}^{I}$ & \multicolumn{1}{|c|}{$\tau_{i}^{u}$} & \multicolumn{1}{c|}{$\tau_{i}^{X}$} & \multicolumn{1}{c|}{$\tau_{i}^{I}$} & \multicolumn{1}{c|}{$\tau_{i}^{u *}$} \\
\hline 1 & 8.40 & -1.06 & 1.39 & 13.23 & -1.06 & 1.39 & 14.47 \\
2 & 12.35 & 2.54 & 1.39 & 9.37 & 2.54 & 1.39 & 11.92 \\
3 & 16.71 & 6.52 & 1.39 & 5.06 & 6.52 & 1.39 & 4.51 \\
4 & 10.97 & 1.28 & 1.39 & 10.58 & 1.28 & 1.39 & 9.43 \\
5 & 11.16 & 1.46 & 1.39 & 10.25 & 1.46 & 1.39 & 9.14 \\
6 & 11.07 & 1.37 & 1.39 & 10.69 & 1.37 & 1.39 & 9.53 \\
7 & 11.17 & 1.46 & 1.39 & 10.58 & 1.46 & 1.39 & 9.43 \\
8 & 11.18 & 1.47 & 1.39 & 10.58 & 1.47 & 1.39 & 10.33 \\
9 & 17.91 & 7.62 & 1.39 & 4.07 & 7.62 & 1.39 & 3.45 \\
10 & 11.29 & 1.57 & 1.39 & 10.18 & 1.57 & 1.39 & 9.08 \\
11 & 8.95 & -0.56 & 1.39 & 12.72 & -0.56 & 1.39 & 11.34 \\
12 & 11.56 & 1.82 & 1.39 & 10.18 & 1.82 & 1.39 & 9.07 \\
13 & 10.23 & 0.60 & 1.39 & 11.30 & 0.60 & 1.39 & 13.64 \\
14 & 12.00 & 2.22 & 1.39 & 9.67 & 2.22 & 1.39 & 11.83 \\
15 & 1.16 & & 1.39 & 0.00 & & 1.39 & 0.0 \\
\hline
\end{tabular}

Steuerreform 3: Einheitlicher Umsatzsteuersatz für alle Unternehmen Steuerreform 5: Einheitlicher effektiver Konsumsteuersatz

hand der effektiven Steuersätze ist darüber hinaus zu ersehen, daß der Übergang zu der neuen Struktur der Konsumsteuersätze eine Äquivalente Variation in Höhe von 1358 Mill. DM erbringt, wobei sich die Terms-of-Trade auf 1.006 erhöhen. Die anschließende Reform der effektiven Außenhandelssteuersätze führt zu einer weiteren Verbesserung der Terms-of-Trade auf 1.009, verbunden mit einem Wohlfahrtsgewinn von 2632 Mill. DM im Vergleich zum Benchmark Equilibrium.

Auf der anderen Seite ergibt auch die Angleichung der effektiven Konsumsteuersätze einen relativ großen Wohlfahrtsgewinn. Mit der in Tabelle 20 als Steuerreform 5 bezeichneten Differentialinzidenz wurden die Umsatzsteuersätze gerade so gewählt, daß der effektive Konsumsteuersatz für alle Güter einheitlich rund 12 Prozent beträgt. Die hierzu 
notwendige Struktur der Umsatzsteuersätze kann der vierten Spalte von Tabelle 20 entnommen werden. Diese Umsatzsteuerreform erbringt mit $E V=3359$ Mill. DM die bislang höchste Wohlfahrtsverbesserung. In sukzessiver Betrachtung zeigt sich auch, daß durch die Angleichung der Konsumsteuersätze bereits eine $E V$ von 2050 realisiert wird - die Terms-of-Trade steigen dabei auf 1.004 -, die sich mit der nachfolgenden Änderung der Außenhandelsbesteuerung (TOT $=1.007)$ nochmals auf 3312 erhöht.

Aus diesen letzten beiden Steuerreformmaßnahmen - insbesondere aus der Zerlegung der Reformen in eine schrittweise Änderung der effektiven Steuersätze - ist die allokative Wirkung der Steuer sehr deutlich zu erkennen. Durch eine bestimmte Wahl der Umsatzsteuersätze ist es möglich, die hauptsächlich von der Produktionssteuer hervorgerufenen allokativen Verzerrungen im Konsumbereich zu kompensieren. Die Vereinheitlichung der Konsumsteuersätze führt gleichzeitig auch zu einer Verbesserung des Weltmarkttauschverhältnisses. Dabei erinnern wir uns, daß mit Steuerreform 4 noch bessere Terms-of-Trade bedingt durch einen höheren Umsatzsteuersatz auf Netto-Importgüter - erzielt werden können als mit Steuerreform 5, allerdings vergrößern sich damit wiederum die Verzerrungen im Konsumbereich. Die unter Effizienzgesichtspunkten beste Struktur der Umsatzsteuersätze ist folglich dann gefunden, wenn ausgehend von den Umsatzsteuersätzen, die einen einheitlichen effektiven Konsumsteuersatz erzeugen, die für Netto-Importgüter relevanten Umsatzsteuersätze solange erhöht werden, bis die Nutzenverbesserung aufgrund der steigenden Terms-ofTrade durch die Nutzeneinbußen aufgrund der steuerbedingten Verzerrungen im Konsumbereich kompensiert werden. In der letzten Spalte von Tabelle 20 sind die aus diesem Vorgehen resultierenden Umsatzsteuersätze $\tau_{i}^{u *}$ angegeben. Sie erbringen eine Wohlfahrtsverbesserung von 3415 Mill. DM. Ohne Veränderung der Kapital- oder der Produktionssteuer ist eine weitere Wohlfahrtssteigerung nicht möglich. 


\section{Sensitivitätsanalyse}

Bereits bei der Kalibrierung der Modellparameter wurde darauf hingewiesen, daß auch mit der spezifischen Wahl der aus der Literatur entnommenen, ökonometrisch geschätzten Parameter eine gewisse Subjektivität verbunden ist. In diesem Abschnitt wollen wir deshalb prüfen, wie sensitiv die Modellergebnisse auf eine Änderung der exogen vorgegebenen Parameterwerte reagieren. Wir werden dabei die zentrale Steuerreformmaßnahme - die Einführung von Nullsteuersätzen - einer systematischen Parametervariation unterziehen.

\subsection{Die Außenhandelselastizitäten}

Bedenkt man, welchen ausgeprägten Einfluß die Terms-of-Trade auf die Wohlfahrtsposition ausüben, liegt es nahe, anstelle des modellierten „großen Landes“ nun ein kleines Land anzunehmen. Für letzteres sind die Terms-of-Trade konstant, d. h. es kann das Weltmarkttauschverhältnis durch sein eigenes Angebot- und Nachfrageverhalten nicht beeinflussen. Dieser Effekt wird durch eine Erhöhung der ausländischen Importangebots- bzw. Exportnachfrageelastizitäten angenähert. Setzen wir $\Theta=-25$ und $\eta=10$, so steigt die Exportnachfrageelastizität von -1.11 auf -7.86 an.

Wie nicht anders zu erwarten war, schwächt sich die positive Wirkung des Terms-of-Trade Effekts durch diese neue Parametersetzung erheblich ab. Zur Verdeutlichung stellen wir in Tabelle 21 die mit der zentralen Parameterwahl bei schrittweisem Übergang zu Nullsteuersätzen erzielten $E V$-Werte denjenigen gegenüber, die sich bei Annahme eines kleinen Landes ergeben.

Man erkennt, daß die Änderung der effektiven Außenhandelsbesteuerung zwar immer noch eine positive Effizienzwirkung ausübt, sie kann den negativen Einfluß der neuen effektiven Konsumsteuersätze aber nicht mehr kompensieren. Mit anderen Worten verursacht die Einräumung eines generellen Vorsteuerabzugsrechts in einem kleinen Land Wohlfahrtsverluste. Dieses Resultat ist einerseits beruhigend, da es die ökonomische Interpretation der mit den einzelnen Simulationen erzielten Ergebnisse nochmals bestätigt, andererseits beunruhigt die Erkenntnis, daß Effizienzwirkung von Nullsteuersätzen sehr stark von der ,richtigen“ Wahl der Außenhandelselastizitäten abhängt. 
Tabelle 21: Effizienzwirkungen des Übergangs zu Nullsteuersätzen für ein großes bzw. kleines Land

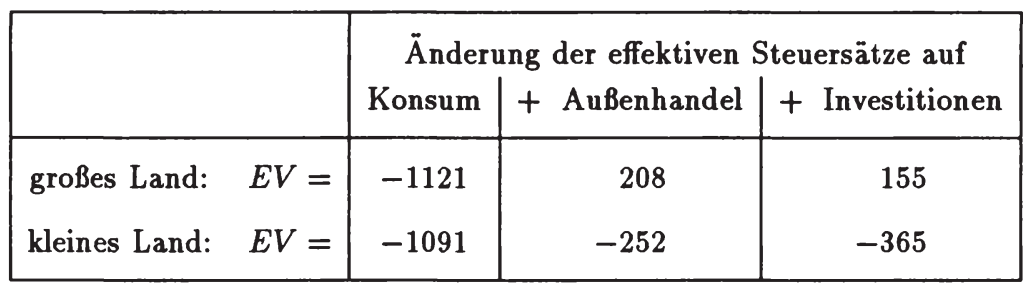

\subsection{Die Arbeitsangebots- und Substitutionselastizitäten}

Die folgenden Parametervariationen befassen sich mit der Vorgabe unterschiedlicher Substitutionselastizitäten $\sigma_{i}$ zwischen Arbeit und Kapital sowie der Änderung der unkompensierten Arbeitsangebotselastizität bezüglich des Lohnsatzes. Um den systematischen Einfluß der Substitutionselastizität auf die Effizienz des Steuersystems feststellen zu können, wird zunächst für alle Unternehmen ein Wert von $\sigma_{i}=\sigma=0.3$ angenommen und anschließend ein Wert von $\sigma=1.8^{138}$.

Wie an anderer Stelle bereits bemerkt wurde, kommen ökonometrische Arbeiten zu recht unterschiedlichen Schätzergebnissen für die unkompensierte Arbeitsangebotselastizität $e$ bezüglich des Lohnsatzes ${ }^{139}$. Im wesentlichen wird dabei ein nahezu unelastisches bis schwach negatives Angebotsverhalten für den (männlichen) Hauptverdiener eines Haushalts festgestellt, während (weibliche) Nebenverdiener ein stark positives Angebotsverhalten zeigen.

Bei der Berechnung von Differentialinzidenzen sind jedoch weniger die unkompensierten Arbeitsangebotselastizitäten ausschlaggebend für die allokative Wirkung einer Steuer, sondern vielmehr die einkommenskompensierten Arbeitsangebotselastizitäten, da die Rückerstattung der erhobenen Steuer in Form von Lump-sum Transfers den Einkommens-

${ }^{138}$ Diese Werte orientieren sich an den niedrigsten bzw. höchsten der in RosKAMP ([1977], S. 369 f.) angegebenen Schätzungen.

139 Ein Überblick über verschiedene Schätzresultate liefert bspw. die Tabelle 6.12 in Ballard/Fullerton/Shoven/Whalley [1985], S. $136 \mathrm{f}$. 
effekt nahezu ausgleicht ${ }^{140}$. Ferner ist bei der Vorgabe von negativen Werten für die unkompensierte Arbeitsangebotselastizität darauf zu achten, daß hierdurch nicht auch die kompensierte einen negativen Wert annimmt und somit einer der fundamentalen Annahmen - einem positiven Slutsky-Term - hinsichtlich des Verhaltens des privaten Haushalts widerspricht ${ }^{141}$.

Aus diesen beiden Erwägungen heraus wählen wir als Beispiel für eine negative unkompensierte Arbeitsangebotselastizität die Parameterkombination $e=-0.04$ und $\xi=1.75$ (die kompensierte Arbeitsangebotselastizität beträgt in diesem Fall 0.28) und im Anschluß daran ein Wertepaar von $e=0$ und $\xi=1.001$, welches eine vollkommen unelastische kompensierte Arbeitsangebotselastizität approximiert. Der Zusammenhang zwischen kompensierter und unkompensierter Arbeitsangebotselastizität sowie dem Verhältnis $\xi$ von gesamter Zeitausstattung zu Freizeit wird in Anhang 5 beschrieben.

Tabelle 22: $\quad E V$-Werte für unterschiedliche Vorgaben von exogenen Parameterwerten

\begin{tabular}{|c|c|}
\hline Geänderte Parameter & $E V$ \\
\hline$\sigma=0.3$ & 81 \\
$\sigma=1.8$ & 194 \\
\hline$e=-0.04 \quad \xi=1.75$ & 187 \\
$e=0.0 \quad \xi=1.001$ & 242 \\
\hline
\end{tabular}

In Tabelle 22 sind die aus den unterschiedlichen Parametervorgaben resultierenden Werte der Äquivalenten Variation zusammenfassend dargestellt. Offensichtlich geht sowohl von den Substitutionselastizitäten als auch von der Arbeitsangebotselastizität eine sehr viel

${ }^{140}$ Der Einkommenseffekt wird nicht gänzlich ausgeglichen, da eine Änderung der Steuersätze in unserem Allgemeinen Gleichgewichtsmodell auch eine Veränderung der Faktorpreise und damit des maximalen Einkommens bewirkt.

${ }^{141}$ Siehe bspw. AtKinson/Stiglitz [1980], S. 34. 
geringere Wirkung auf die Effizienz der betrachteten Steuerreformmaßnahme aus, als von den zuvor betrachteten Außenhandelselastizitäten.

Da die Unternehmen bei einer hohen Substitutionselastizität zwischen Arbeit und Kapital mit einer relativ stärkeren Umschichtung der Faktoreinsatzmengen auf eine Änderung der Faktorpreise reagieren, erscheint es plausibel, daß die Allokationseffekte bei großen $\sigma$-Werten vergleichsweise stärker ausfallen als bei kleineren. Zur Einschätzung der Effizienzwirkung von unterschiedlichen Kombinationen von $e$ und $\xi$ ist die theoretische Erkenntnis hilfreich, daß höhere absolute Werte der unkompensierten Arbeitsangebotselastizität zu größeren steuerbedingten Wohlfahrtsverlusten führen ${ }^{142}$. Dementsprechend zeigt unsere Zerlegung des Übergangs zu Nullsteuersätzen in eine schrittweise Änderung der effektiven Steuersätze, daß die Reform der effektiven Konsumsteuersätze bei der ersten in Tabelle 22 angegebenen Parameterkombination zu einer Äquivalenten Variation von -1037, bei der zweiten Kombination zu einer $E V$ von -981 Mill. DM führt. Insgesamt betrachtet, haben jedoch weder die Substitutionselastizitäten noch die Arbeitsangebotselastizitäten einen allzu großen Einfluß auf die erzielten Ergebnisse.

\subsection{Das LES-Nachfragesystem}

Eine andere Qualität von Sensitivitätsanalyse kommt in der nun folgenden Modifikation des Modells zum Ausdruck. Bei der Beschreibung des theoretischen Modellteils wurde darauf hingewiesen, daß bereits die Wahl der funktionalen Form für die Nutzenfunktion bestimmte Restriktionen hinsichtlich des Konsumentenverhaltens impliziert. Wir wollen deshalb - als Alternative zur bisherigen Struktur des Nutzenbaumes - den Cobb-Douglas Nutzenast durch eine LES- bzw. Stone/GearyNutzenfunktion ersetzen. Die hierzu notwendigen Änderungen des Modells, einschließlich der im Haushaltsbereich neu zu kalibrierenden $\mathrm{Pa}$ rameter, werden in Anhang 6 skizziert.

Die Konsumgüter-Nachfragefunktionen des Linearen Ausgabensystems sind durch

${ }^{142}$ Siehe bspw. HAUSMANN [1985]. 


$$
C_{i}=\gamma_{i}+\frac{\alpha_{i}\left(Y^{v e r}-\Gamma\right)}{p_{i}}
$$

gegeben, wobei $\gamma_{i}$ die konsumierte Mindestmenge an Güterart $i$ und $\Gamma=\sum_{i=1}^{14} p_{i} \gamma_{i}$ die damit insgesamt verbundenen Mindestausgaben bzw. das Subsistenzminimum kennzeichnen. Entsprechend kommt auch den $\alpha_{i}$ eine leicht veränderte Bedeutung zu. Sie bestimmen den Anteil der diskretionären - d. h. den Mindestkonsum übersteigenden - Ausgaben $p_{i} C_{i}-p_{i} \gamma_{i}$ für Gut $i$ am diskretionären Einkommen $Y^{\text {ver }}-\Gamma$.

Unterstellt man dieses Nachfrageverhalten, so resultiert die Einräumung eines allgemeinen Vorsteuerabzugsrechts in einer Verbesserung der Wohlfahrt von 341 Mill. DM. Dieser im Vergleich zum CES/ Cobb-Douglas System (bei zentraler Parameterwahl für beide Versionen) höhere Wohlfahrtsgewinn erklärt sich im wesentlichen aus der geringeren Flexibilität der Konsumgüternachfrage. Da im LES-System nur noch ein Teil der nachgefragten Konsumgütermengen von den (Konsumenten-) Preisen abhängt, verursacht eine steuerbedingte Preisänderung vergleichsweise geringere Verzerrungen der Güterallokation. Wie aus der ersten Zeile von Tabelle $23 \mathrm{zu}$ ersehen ist, führt die Änderung der effektiven Konsumsteuersätze deshalb zu relativ geringeren Wohlfahrtseinbußen wie bei einem Cobb-Douglas Nutzenast. Um auch eher skeptische Leser von dieser Interpretation zu überzeugen, wurden die Mindestmengen kurzerhand verdoppelt und anschließend dieselben Simulatonsläufe nochmals durchgeführt. Die sich ergebenden Wohlfahrtseffekte sind in der zweiten Zeile von Tabelle 23 ausgewiesen.

Tabelle 23: Effizienzwirkungen des Übergangs zu Nullsteuersätzen für das LES-Nachfragesystem

\begin{tabular}{|l|c|c|c|}
\hline & \multicolumn{3}{|c|}{ Änderung der effektiven Steuersätze auf } \\
Mindestmengen & Konsum & + Außenhandel & + Investitionen \\
\hline Standard: $E V=$ & -1024 & 426 & 341 \\
Erhöht: $\quad E V=$ & -523 & 1378 & 1254 \\
\hline
\end{tabular}


Aus der Theorie der optimalen Besteuerung ist aber auch bekannt, daß hinsichtlich der LES-Nutzenfunktion ein einheitlicher effektiver Konsumsteuersatz nicht unbedingt die effizienteste Form der Besteuerung sein muß. Die optimalen Steuersätze sind in etwa umgekehrt proportional zur entsprechenden Einkommenselastizität der Nachfrage zu wählen ${ }^{143}$. Somit kann anhand der Struktur der effektiven Konsumsteuersätze allein nicht mehr beurteilt werden, ob eine Änderung der Steuersätze positive oder negative Wirkungen im Konsumbereich verursacht. Eine Vereinheitlichung der Umsatzsteuersätze könnte folglich im LES-System (qualitativ) andere Effizienzeffekte hervorrufen.

Es zeigt sich jedoch, daß die Ergebnisse nicht wesentlich von den in Abschnitt (4) erzielten abweichen. Die partielle Vereinheitlichung der Umsatzsteuersätze führt unter Beibehaltung des Vorsteuerabzugsverbots zu einer Äquivalenten Variation in Höhe von -29 Mill. DM, während bei zusätzlicher Einräumung des Vorsteuerabzugsrechts ein Wohlfahrtszuwachs von 355 Mill. DM erzielt wird. Überraschenderweise ist dieser Wohlfahrtsgewinn nahezu mit demjenigen identisch, der durch den Übergang zu Nullsteuersätzen bei gleichzeitiger aufkommensneutraler Anhebung der ursprünglichen Steuersätze erreicht werden kann. Ein allgemeiner Umsatzsteuersatz in Höhe von $9.6 \mathrm{v}$. H. erbringt schließlich etwas höhere Effizienzgewinne $(E V=2854)$ als im CES/Cobb-Douglas System.

Man könnte nun wiederum nach der optimalen Struktur der Umsatzsatzsteuersätze fragen, wobei ein Verfahren analog zu dem im vorletzten Abschnitt beschriebenen anzuwenden wäre. Man bestimmt diejenigen Umsatzsteuersätze, die eine zu den entsprechenden Einkommenselastizitäten umgekehrt proportionale Struktur der effektiven Konsumsteuersätze erzeugen und versucht anschließend durch Erhöhung der Umsatzsteuersätze auf Netto-Importgüter die Terms-of-Trade zu verbessern. Wir werden jedoch auf diese „Übung“ verzichten. Die wichtigsten Erkenntnisse sind bereits zusammengetragen und werden im folgenden Abschnitt nochmals zusammenfassend dargestellt.

143 Siehe hierzu auch ATKINSON/Stiglitz [1972] oder HARris/MACKINNON [1979]. 


\section{Abschließende Bemerkungen}

Nahezu alle Simulationen mit unterschiedlichen Parametervorgaben oder einer alternativen Struktur der Nutzenfunktion ergaben, daß mit der Einführung eines allgemeinen Vorsteuer abzugsrechts bei gleichzeitiger Anhebung der Umsatzsteuersätze ein zwar nur moderater, aber doch signifikanter Effizienzgewinn erzielt wird. Mit der StandardParameterwahl sowie der Annahme einer CES/Cobb-Douglas Nutzenfunktion beträgt der in Einkommensäquivalenten gemessene Wohlfahrtszuwachs rund $7.2 \mathrm{v}$. T. des mit dem Nachholeffekt erzielten Umsatzsteueraufkommens und immerhin rund $15.3 \mathrm{v}$. T. bei Unterstellung einer CES/LES Nutzenfunktion.

Dagegen führt die Vereinheitlichung der Umsatzsteuersätze (in der zentralen Modellspezifikation) nur dann zu einer Wohlfahrtsverbesserung, wenn es möglich ist, tatsächlich alle Sektoren zu einem allgemeinen Satz zu besteuern. Werden die bislang geltenden Umsatzsteuerbefreiungen hingegen beibehalten; so resultiert aus der Angleichung der Steuersätze ein leichter Wohlfahrtsverlust in Höhe von -142 Mill. DM. Letzterer könnte beispielsweise dadurch kompensiert werden, wenn man diese Steuerreformmaßnahme mit der Einführung einer allgemeinen Vorsteuerabzugsberechtigung verbindet. Erst der allgemeine Umsatzsteuersatz, angewendet auf alle Sektoren der Volkswirtschaft, erbringt mit $E V=2693$ einen wesentlichen Effizienzgewinn.

Diese zentralen Ergebnisse der numerischen Berechnungen ändern sich nicht signifikant, falls die exogen gesetzten Substitutionselastizitäten zwischen Arbeit und Kapital variiert werden. Dabei zeigte sich, daß eine höhere Reagibilität des Faktoreinsatzverhältnisses in Bezug auf Preisänderungen tendenziell zu größeren Allokationseffekten führt. Ebenso konnten größere Effizienzwirkungen - in einer etwas ausgeprägteren Form - bei einer Senkung der einkommenskompensierten Arbeitsangebotselastizität bezüglich des Lohnsatzes festgestellt werden. Erhöht man jedoch die (ausländische) Exportnachfrage- bzw. die Importangebotselastizität, ändert sich nicht nur der absolute Wert der Äquivalenten Variation, sondern auch das Vorzeichen und somit das qualitative Ergebnis für unsere zentrale Umsatzsteuerreform.

Wurde mit der Standard-Parameterwahl bei einem Übergang zu Nullsteuersätzen ein Wohlfahrtsgewinn berechnet, so ist nun ein mehr 
als doppelt so großer Wohlfahrtsverlust zu verzeichnen. Der Grund hierfür ist in einer weniger günstigen Entwicklung der Export- bzw. Importmengen zu sehen. Damit kommen wir unmittelbar zu einer Zusammenfassung der Ursachen, die letztendlich die errechneten Allokationseffekte bewirken.

Ohne die in einem Gleichgewichtssytem über den Marktmechanismus zwangsläufig durch die nominellen Umsatzsteuersätze hervorgerufenen Preisänderungen einzubeziehen, verursacht die von Umsatzsteuerbefreiungen ausgehende versteckte Besteuerung eine direkte Verzerrung aller Produzentenpreise. Ähnlich gelagerte Verzerrungen gehen auch von den Produktions- und den Kapitalsteuern aus ${ }^{144}$. Diese Verzerrungen zusammengenommen beeinflussen den Außenhandel, die Investitionsnachfrage - damit verbunden das Faktoreinsatzverhältnis - und gemeinsam mit den nominalen Umsatzsteuersätzen die Konsumgüternachfrage. Sie kommen im wesentlichen in den (erweiterten) effektiven Steuersätzen zum Ausdruck.

Die Effizienz eines bestimmten Umsatzsteuersystems kann deshalb anhand der effektiven Steuersätze - unter Zuhilfenahme einfacher theoretischer Erkenntnisse - beurteilt werden. Insbesondere decken die effektiven Steuersätze auf, daß Umsatzsteuerbefreiungen eine nutzenmäßige Kompensation der von den übrigen Steuern ausgehenden Verzerrungen im Konsumbereich bewirken und ebenso die im Unternehmensbereich bestehende Fehlallokation zwischen den Produktionsfaktoren abschwächen. Gleichzeitig beeinflussen Umsatzsteuerbefreiungen durch relativ starke implizite Subventionen der Netto-Importgüter, aber auch das Weltmarkttauschverhältnis zuungunsten des Inlands, was schließlich den Ausschlag für die insgesamt negative Wirkung von Befreiungen gibt.

Selbst unter Effizienzgesichtspunkten allein wäre es jedoch etwas voreilig, aus diesem Resultat ein vehementes Plädoyer für die Abschaffung des Vorsteuerabzugsverbots abzuleiten. Denn gerade der starke Einfluß, den die Änderung der effektiven Außenhandelsbesteuerung ausübt, ist nicht zuletzt darauf zurückzuführen, daß die theoretische

144 Wobei die Kapitalsteuern darüber hinaus das Faktorpreisverhältnis stören. 
Modellstruktur eine ausgeglichene Leistungsbilanz impliziert ${ }^{145}$. Zieht man beispielsweise internationale Kapitalmärkte in Betracht, so ist zu vermuten, daß die Terms-of-Trade nicht in dem Maße verändert werden, wie sie die Simulationsrechnung ergibt. Ein Ungleichgewicht der Leistungsbilanz könnte dann auch durch Kapitalbewegungen ausgeglichen werden kann. Letztere üben ihrerseits einen Einfluß auf die relativen Faktorpreise aus, der im Modell nicht berücksichtigt werden konnte.

Während der Terms-of-Trade Effekt demnach tendenziell überbewertet ist, wird der Verzerrung des Faktoreinsatzverhältnisses ein zu niedriger Stellenwert eingeräumt. Die von der indirekten Besteuerung der Investitionen verursachten Effizienzwirkungen manifestieren sich lediglich in der Fehlalloktion der Ressourcen in einer bestimmten Periode. Die zusätzlichen Wohlfahrtseffekte, die mit einem veränderten gleichgewichtigen Wachstumspfad sowie der auf dem Weg zum neuen Steady State entstehenden Transitionskosten verbunden sind, werden nicht erfaßt. Ferner ist zu bedenken, daß auch die Nachfrage nach Zwischenprodukten in unserer eher langfristigen Betrachtung nicht die modellierte Konstanz aufweist.

Angesichts dieser Liste von einschränkenden Annahmen entsteht unmittelbar die Frage, wie sinnvoll eine derartige Analyse überhaupt ist. Die Liste könnte durchaus noch um Einiges erweitert werden, wie beispielsweise der Hinweis auf die einfache Form der Nutzenfunktion oder die Vernachlässigung von Finanzmärkten, um nur zwei weitere Punkte zu nennen. Dem ist jedoch entgegenzuhalten, daß erst durch die Anwendung der Empirischen Allgemeinen Gleichgewichtsanalyse und der daraus im konkreten Fall gewonnenen Erkenntnisse die relative Bedeutung der einzelnen Vereinfachungen ermessen werden kann. So stehen einige der oben genannten Einschränkungen auf der Agenda für zukünftige Forschung.

Darüber hinaus besteht das Ziel der EAG-Analyse auch nicht darin, ein perfektes Abbild der Realität zu schaffen, um anschließend bestimmte Steuerreformmaßnahmen auf Heller und Pfennig exakt zu berechnen, sondern man versucht, auf einer soliden theoretischen Grundlage, Erkenntnisse aufzudecken, die mit anderen analytischen Instrumenten nicht ohne Weiteres erkennbar wären. Sie tragen damit zu ei-

${ }^{145}$ Siehe auch Goulder/Shoven/WhaLLEY [1983]. 
nem besseren Verständnis der ökonomischen Wirkungszusammenhänge bei und liefern gleichzeitig neue Anreize für eine fundiertere wirtschaftspolitische Diskussion über die betrachtete Steuerreform. 


\section{Anhang}

Anhang 1: Liste der steuerfreien Umsätze ${ }^{146}$

1. Ausfuhrlieferung und Lohnveredelung an Gegenständen der Ausfuhr;

2. Umsätze der Seeschiffahrt und Luftfahrt;

3. Grenzüberschreitende Beförderungen im internationalen Eisenbahnfrachtverkehr;

4. Lieferungen von Gold an die Zentralbanken;

5.a Vermittlung der unter 1-4 fallenden Umsätze;

b Grenzüberschreitende Beförderung von Personen mit Luftfahrzeugen oder Seeschiffen;

c Umsätze, die ausschließlich im Außengebiet bewirkt werden;

6.a Leistungen der Deutschen Bundesbahn;

b Leistungen an Vertragsparteien der NATO;

c Durchfuhr von Gegenständen;

7. Entfallen

8.a Gewährung, Vermittlung und Verwaltung von Krediten;

b Umsätze und die Vermittlung der Umsätze von gesetzlichen Zahlungsmitteln;

c Umsätze von Geldforderungen, Optionsgeschäfte mit Geldforderungen und Vermittlung solcher Umsätze;

d Typische Bankumsätze;

e Umsätze von Wertpapieren und Optionsgeschäfte mit Wertpapieren, sowie die in diesen Bereich fallende Vermittlungs-, Verwahrungs- und Verwaltungsleistungen;

f Umsätze von Anteilen an Gesellschaften und anderen Vereinigungen sowie deren Vermittlung;

g Übernahme von Verbindlichkeiten, Bürgschaften und ähnlichen Sicherheiten sowie die Vermittlung solcher Umsätze;

h Die Verwaltung von Kapitalanlagegesellschafts-Sondervermögen;

${ }^{146}$ Die Liste der steuerfreien Umsätze umfaßt die in $§ 4$ UStG aufgeführten Umsatzarten, wobei jedoch nicht der genaue Wortlaut, sondern lediglich eine Kurzbeschreibung wiedergegeben ist. 
i Umsätze von im Erhebungsgebiet gültigen amtlichen Wertzeichen zum aufgedruckten Wert;

j Die Beteiligung als stiller Gesellschafter;

9.a Umsätze, die unter das Grunderwerbsteuergesetz fallen;

b Umsätze, die unter das Rennwett- und Lotteriegesetz fallen;

10.a Leistungen auf Grund eines Versicherungsverhältnisses;

b Leistungen, die darin bestehen, daß anderen Personen Versicherungsschutz verschafft wird;

11. Umsätze aus der Tätigkeit als Bausparkassenvertreter, Versicherungsvertreter und Versicherungsmakler;

12.a Die Vermietung und Verpachtung von Grundstücken;

b Die Überlassung von Grundstücken;

c Die Übertragung des Nutzungsrechts an Grundstücken;

13. Instandhaltungs- und Instandsetzungsleistungen, die die Gemeinschaften von Wohnungseigentümer an die Wohnungseigentümer erbringen;

14. Umsätze aus der Tätigkeit als Arzt, Zahnarzt, Heilpraktiker, usw.;

15. Umsätze der gesetzlichen Träger der Sozialversicherung;

16. Umsätze, die mit dem Betrieb von Krankenhäusern, Diagnosekliniken u. ä. verbunden sind;

17.a Die Lieferung von menschlichen Organen, menschlichem Blut und Frauenmilch;

b Die Beförderung von Kranken und verletzten Personen;

18. Die Leistungen von Verbänden der Wohlfahrtspflege;

19.a Umsätze der Blinden mit nicht mehr als zwei Arbeitnehmern;

b Leistungen von Blindenwerkstätten;

20.a Umsätze von kulturellen Einrichtungen des Bundes (Theater, Orchester, usw.);

b Veranstaltungen der unter 20.a fallenden Einrichtungen;

21. Leistungen von anerkannten Einrichtungen, die dem Schul- und Bildungszweck dienen;

22.a Wissenschaftliche Veranstaltungen von juristischen Personen des öffentlichen Rechts;

b Kulturelle und sportliche Veranstaltungen der unter a) genannten Einrichtungen;

23. Beherbergungs- und Beköstigungsleistungen im Rahmen der Fortbildung Jugendlicher; 
24. Die Leistungen des Deutschen Jugendherbergswerkes;

25. Leistungen der förderungswürdigen Träger der freien und öffentlichen Jugendhilfe;

26. Ehrenamtliche Tätigkeiten;

27. Gestellung von Mitgliedern geistlicher Genossenschaften und Angehörigen von Mutterhäusern für gemeinnützige, mildtätige kirchliche oder schulische Zwecke;

28.a Die Lieferung von Gegenständen, die ausschließlich für eine nach Nummer 7 bis 27 aufgelistete Tätigkeit verwendet werden;

b Verwendung von Gegenständen außerhalb des Unternehmens für eine nach Nummer 7 bis 27 verwendete Tätigkeit und vom Unternehmer als Eigenverbrauch versteuert. 
Anhang 2: Länder, die eine Netto-Allphasenumsatzsteuer einführten unter Angabe der Verbrauchsteuer, welche die Umsatzsteuer ersetzte

\begin{tabular}{|c|c|c|c|}
\hline & $\begin{array}{l}\text { Date VAT } \\
\text { Introduced }\end{array}$ & $\begin{array}{l}\text { Sales Taxes } \\
\text { Mainly Replaced }\end{array}$ & $\begin{array}{l}\text { Designed Eff } \\
\text { On Revenue }\end{array}$ \\
\hline Argentinia & Jan. 1975 & $\begin{array}{l}\text { Wholesale sales and provin- } \\
\text { cial cascade turnover tax }\end{array}$ & Equal yield \\
\hline Austria & Jan. 1973 & Cascade wholesale & Equal yield \\
\hline Belgium & Jan. 1971 & Cascade wholesale & Equal yield \\
\hline Bolivia & Nov. 1973 & Multistage ring system & $\begin{array}{l}\text { Equal yield } \\
\text { or increase }\end{array}$ \\
\hline Brazil *) & Jan. 1967 & $\begin{array}{l}\text { Cascade tax on sales and } \\
\text { consignments }\end{array}$ & Equal yield \\
\hline Chile & Mar. 1975 & $\begin{array}{l}\text { Cascade turnover, manufac- } \\
\text { turers tax, and special } \\
\text { luxury tax }\end{array}$ & Equal yield \\
\hline Columbia & Jan. 1965 & No previous sales tax & Increase \\
\hline Costa Rica & Jan. 1975 & Multistage ring system & Increase \\
\hline Côte d'Ivoire & Jan. 1960 & Manufacturers VAT & Equal yield \\
\hline Denmark & July 1967 & Wholesale & Increase \\
\hline Dominican Rep. & Nov. 1983 & No previous sales tax & Increase \\
\hline Ecuador & Aug. 1970 & Turnover taxes on mining & Increase \\
\hline France & Jan. 1968 & $\begin{array}{l}\text { An earlier and less sophisti- } \\
\text { cated VAT }\end{array}$ & Equal yield \\
\hline $\begin{array}{l}\text { Germany, Fed. } \\
\text { Rep. of }\end{array}$ & Jan. 1968 & Cascade retail & Equal yield \\
\hline Grenada & Apr. 1986 & $\begin{array}{l}\text { Stamp duties and taxes on } \\
\text { services }\end{array}$ & Equal yield \\
\hline Greece & Jan. 1987 & $\begin{array}{l}\text { Turnover tax, stamp duties, } \\
\text { and special import levy }\end{array}$ & Equal yield \\
\hline Guatemala & Aug. 1983 & $\begin{array}{l}\text { Stamp duty on sales, } \\
\text { services and imports }\end{array}$ & $\ldots$ \\
\hline Haiti & Nov. 1982 & Seventy-nine excises & Equal yield \\
\hline Honduras & Jan. 1976 & Single-stage ring system & Increase \\
\hline Hungary & Jan. 1988 & $\begin{array}{l}\text { Production and turnover } \\
\text { taxes }\end{array}$ & Increase \\
\hline Indo & Apr. 1985 & $\begin{array}{l}\text { Manufacturers ring system } \\
\text { with eight rates }\end{array}$ & vield \\
\hline
\end{tabular}




\begin{tabular}{|c|c|c|c|}
\hline & $\begin{array}{l}\text { Date VAT } \\
\text { Introduced }\end{array}$ & $\begin{array}{l}\text { Sales Taxes } \\
\text { Mainly Replaced }\end{array}$ & $\begin{array}{l}\text { Designed Effect } \\
\text { On Revenue }\end{array}$ \\
\hline Ireland & Nov. 1972 & Wholesale and retail sales & Equal yield \\
\hline Israel & July 1976 & Various sales & Increase \\
\hline Italy & Jan. 1973 & $\begin{array}{l}\text { General and local govern- } \\
\text { ment sales }\end{array}$ & Equal yield \\
\hline Japan & Apr. 1989 & No sales tax & Increase \\
\hline Korea & July 1977 & $\begin{array}{l}\text { Eight sales taxes represen- } \\
\text { ting } 40 \text { percent of revenue }\end{array}$ & Equal yield \\
\hline Luxembourg & Jan. 1970 & Cascade wholesale & Equal yield \\
\hline Madagascar & Jan. 1969 & Cascade production & Increase \\
\hline Mexico & Jan. 1980 & $\begin{array}{l}\text { Cascade production and } \\
\text { revoked } 18 \text { selective } \\
\text { sales taxes }\end{array}$ & $\begin{array}{l}\text { Equal yield } \\
\text { or increase }\end{array}$ \\
\hline Morocco & Jan. 1962 & Cascade production & Equal yield \\
\hline Netherlands & Jan. 1969 & Cascade wholesale & Equal yield \\
\hline New Zealand & May 1986 & Wholesale tax & $\begin{array}{l}\text { Yield extra } \\
\text { revenue }\end{array}$ \\
\hline Nicaragua & Aug. 1978 & Multistage ring system & Equal yield \\
\hline Niger & Jan. 1986 & Cascade manufacturers & $\begin{array}{l}\text { Yield extra } \\
\text { revenue }\end{array}$ \\
\hline Norway & Jan. 1970 & $\begin{array}{l}\text { Sales taxes on } 65 \text { percent } \\
\text { of consumption }\end{array}$ & Loss \\
\hline Panama & Jan. 1976 & No sales tax & Increase \\
\hline Peru & Jan. 1973 & $\begin{array}{l}\text { Cascade production and } \\
\text { stamp tax }\end{array}$ & Increase \\
\hline Philippines & Jan. 1988 & Eight sales and stamp taxes & Increase \\
\hline Portugal & Jan. 1986 & Single-stage wholesale & Equal yield \\
\hline Senegal & Mar. 1961 & Manufacturers VAT 6 & Equal yield \\
\hline Spain & Jan. 1986 & $\begin{array}{l}\text { Cascade production tax } \\
\text { and } 20 \text { other sales taxes }\end{array}$ & $\ldots$ \\
\hline Sweden & Jan. 1969 & $\begin{array}{l}\text { Retail sales tax and capital } \\
\text { goods tax }\end{array}$ & Equal yield \\
\hline $\begin{array}{l}\text { Taiwan, Province } \\
\text { of China }\end{array}$ & Apr. 1986 & $\begin{array}{l}\text { Cascade retail tax and } \\
\text { stamp duty }\end{array}$ & Equal yield \\
\hline Turkey & Jan. 1985 & $\begin{array}{l}\text { Eight production taxes and } \\
\text { other duties on goods and } \\
\text { services }\end{array}$ & Equal yield \\
\hline
\end{tabular}




\begin{tabular}{llll}
\hline & $\begin{array}{l}\text { Date VAT } \\
\text { Introduced }\end{array}$ & $\begin{array}{l}\text { Sales Taxes } \\
\text { Mainly Replaced }\end{array}$ & $\begin{array}{l}\text { Designed Effect } \\
\text { On Revenue }\end{array}$ \\
\hline $\begin{array}{l}\text { United Kingdom } \\
\text { Uruguay }\end{array}$ & $\begin{array}{l}\text { Apr. 1973 } \\
\text { Jan. 1968 }\end{array}$ & $\begin{array}{l}\text { Multirate wholesale } \\
\text { Manufacturers single-stage } \\
\text { tax and a cascade turnover } \\
\text { tax }\end{array}$ & $\begin{array}{l}\text { Loss } \\
\end{array}$ \\
& & \\
\hline
\end{tabular}

*) Note Brazil introduced a federal VAT on interstate transactions and a state VAT on intrastate sales (ICM); however, the Federal Government determines the tax base and rates.

Quelle: TAIT [1988], S. $10 \mathrm{ff}$.

Die Tabelle wurde weitgehend im Original belassen, mit Ausnahme der Länder, in denen die Einführung einer Netto-Allphasen-Umsatzsteuer vorgeschlagen, jedoch bislang noch nicht realisiert wurde.

Die Bezeichnungen cascade production tax, cascade wholesale tax und cascade retail sales tax stehen respektive für eine mehrphasige Bruttoumsatzsteuer auf Herstellungsebene, eine Einzelhandel-Vor-Umsatzsteuer und eine Bruttoallphasen-Umsatzsteuer. Production tax, wholesale tax und retail sales tax bezeichnen hingegen einphasige Produktion-,Grossisten- und Einzelhandelsteuern. Der Ausdruck ring system weist darauf hin, daß ein in Teilabschnitt (2.1) beschriebenes Registrierungsverfahren angewandt wurde. 
Anhang 3: Funktionelle Formen und Parameterwerte für das numerische Beispiel aus Kapitel II, Teilabschnitt (4.2)

Haushalt

Konsumgüternachfrage: $\quad C_{i}=\frac{\alpha_{i}}{p_{i}}(w \bar{L}+r \bar{K}+T R)$

Unternehmen

Wertschöpfungsfunktion:

$$
f(\cdot)=\phi_{i} L_{i}^{\sigma_{i}} K_{i}^{1-\sigma_{i}}
$$

Faktornachfrage:

$$
\begin{aligned}
K_{i} & =\frac{1}{\phi_{i}}\left[\frac{1-\sigma_{i}}{\sigma_{i}} \frac{w}{r}\right]^{\sigma_{i}} a_{0 i} Q_{i} \\
L_{i} & =\frac{1}{\phi_{i}}\left[\frac{\sigma_{i}}{1-\sigma_{i}} \frac{r}{w}\right]^{1-\sigma_{i}} a_{0 i} Q_{i}
\end{aligned}
$$

\section{Gleichgewichtsbedingungen}

Faktormärkte:

$$
\begin{gathered}
\bar{L}=\sum_{i=1}^{3} L_{i} \quad \bar{K}=\sum_{i=1}^{3} K_{i} \\
Q_{i}=C_{i} \sum_{j=1}^{3} a_{i j} Q_{j}
\end{gathered}
$$

Gütermärkte: 
Staatliche Budgetbeschränkung im Umsatzsteuer-Gleichgewicht Konsumsteuer-Gleichgewicht

$$
\begin{aligned}
& T R=\sum_{i=1}^{3} \tau_{i}^{u} q_{i} C_{i}+ \\
& \tau_{1}^{u} q_{1} a_{13} Q_{3}+\tau_{2}^{u} a_{23} Q_{3}
\end{aligned}
$$$$
T R=\sum_{i=1}^{3} \tau_{i}^{c} \tilde{q}_{i} C_{i}
$$

mit $\quad p_{i}=\left(1+\tau_{i}^{u}\right) q_{i}$

$$
p_{i}=\left(1+\tau_{i}^{c}\right) \tilde{q}_{i}
$$

Gleichgewichts-Preisvektor

Faktorpreise:

Güterpreise:

Haushalt

$\alpha_{1}=0.409$

$\alpha_{2}=0.358$

$\alpha_{3}=0.233$

$$
w=1.0 \quad r=1.0
$$

$p_{1}=1.1$

$$
p_{2}=1.1
$$$$
p_{3}=1.0
$$

\section{Parameterwerte}

$\begin{array}{ccc}\text { Haushalt } & \text { Unternehmen } \\ \alpha_{1}=0.409 & \sigma_{1}=0.486 & \phi_{1}=1.999 \\ \alpha_{2}=0.358 & \sigma_{2}=0.467 & \phi_{2}=1.996 \\ \alpha_{3}=0.233 & \sigma_{1}=0.593 & \phi_{1}=1.965\end{array}$




\section{Anhang 4: Die Berechnung der Äquivalenten Variation für eine CES-Nutzenfunktion.}

Die zur Berechnung der Äquivalenten Variation

$$
E V=\varrho\left(\mathbf{p}_{0} ; \mathbf{p}_{1}, Y_{1}\right)-Y_{0}
$$

benötigte Kompensationsfunktion $\varrho(\cdot)$ kann aus der Ausgabenfunktion bestimmt werden. Es gilt

$$
\varrho\left(\mathbf{p}_{\mathbf{0}} ; \mathbf{p}_{\mathbf{1}}, Y_{1}\right) \equiv E\left(\mathbf{p}_{\mathbf{0}}, V\left(\mathbf{p}_{\mathbf{1}}, Y_{1}\right)\right)
$$

mit $E(\cdot)$ als der Ausgabenfunktion und $V(\cdot)$ als der indirekten Nutzenfunktion.

Letztere wird durch Einsetzen der Nachfragefunktionen nach Freizeit $F$ und Konsum $C$ mit

$$
\begin{aligned}
F & =\frac{\theta}{\Omega w^{\nu}} Y^{\max } \\
C & =\frac{1-\theta}{\Omega p^{\nu}} Y^{\max } \\
\text { und } \quad \Omega & =\left[(1-\theta) p^{1-\nu}+\theta w^{1-\nu}\right]
\end{aligned}
$$

in die direkte Nutzenfunktion

$$
U(F, C)=\left[(1-\theta)^{\frac{1}{\nu}} C^{\frac{\nu-1}{\nu}}+\theta^{\frac{1}{\nu}} F^{\frac{\nu-1}{\nu}}\right]^{\frac{\nu}{\nu-1}}
$$

ermittelt. Nach einigen Umformungen erhalten wir für die indirekte Nutzenfunktion

$$
\begin{aligned}
V(p, w, Y) & =\left[(1-\theta) p^{1-\nu}+\theta w^{1-\nu}\right]^{\frac{\nu}{\nu-1}} \frac{Y^{\max }}{\Omega} \\
& =\Omega^{\frac{1}{\nu-1}} Y^{\max }
\end{aligned}
$$


wobei sich der allgemeine Preisvektor $\mathbf{p}$ in diesem Fall aus dem Preisindex $p$ für das Composite Commodity Konsum und dem Lohnsatz $w$ zusammensetzt.

Verwendet man diese Beziehung in der benötigten Ausgabenfunktion bzw. der Berechnungsformel der Aquivalenten Variation, ergibt sich

$$
\begin{aligned}
& E V=\Omega_{0}^{\frac{1}{1-\nu}}\left(\Omega_{1}^{\frac{1}{\nu-1}} Y_{1}^{\max }\right)-Y_{0}^{\max } \\
& =\left[\Omega_{1}^{\frac{1}{\nu-1}} Y_{1}^{\max }-\Omega_{0}^{\frac{1}{\nu-1}} Y_{0}^{\max }\right] \Omega_{0}^{\frac{1}{1-\nu}} .
\end{aligned}
$$

Subscript 0 repräsentiert hierbei analog zur allgemeinen Definition die Größen im Ausgangsgleichgewicht, Subscript 1 die Größen im neuen Gleichgewicht. Aus der letzten Gleichung läßt sich unmittelbar nach Ausklammern von $1 / v_{0}$ und unter Berücksichtigung der Identität $V_{i}\left(p_{i}, w_{i}, Y_{i}^{\text {max }}\right) \equiv U_{i}\left(C_{i}, F_{i}\right)$ mit $i=0,1$ die in Kapitel IV, Teilabschnitt (1.4) angegebene Formel

$$
E V=\frac{U_{1}-U_{0}}{U_{0}} Y_{0}^{\max }
$$

gewinnen. 


\section{Anhang 5: Die Beziehung zwischen kompensierter und un- kompensierter Arbeitsangebotselastizität bezüg- lich des Lohnsatzes}

Zerlegt man die marginale Änderung des Arbeitsangebots bezüglich des Lohnsatzes $\partial L / \partial w$ in die Slutzky-Terme, so erhält man

$$
\frac{\partial L}{\partial w}=\left.\frac{\partial L}{\partial w}\right|_{u}+L \frac{\partial L}{\partial Y}
$$

bzw. in Form von Elastizitäten ausgedrückt

$$
e=e_{u}+\frac{w L}{Y} e_{y}
$$

mit

$$
e:=\frac{\partial L}{\partial w} \frac{w}{L} ; \quad e_{u}:=\left.\frac{\partial L}{\partial w} \frac{w}{L}\right|_{u} \quad \text { und } \quad e_{y}:=\frac{\partial L}{\partial Y} \frac{Y}{L}
$$

Dabei kann wiederum $e_{y}$ über

$$
\begin{aligned}
e_{y}=\frac{\partial(E-F)}{\partial Y} \frac{Y}{E-F} & =-\frac{\partial F}{\partial Y} \frac{Y}{E-F} \\
& =-\frac{\partial F}{\partial Y} \frac{Y}{F} \frac{F}{E-F} \\
& =-\frac{\partial F}{\partial Y} \frac{Y}{F}(\xi-1)
\end{aligned}
$$

als eine Beziehung zwischen der Einkommenselastizität bezüglich der Freizeitnachfrage und dem Verhältnis $\xi$ von gesamter Zeitausstattung zu Freizeit beschrieben werden. Da die Einkommenselastizität der Freizeitnachfrage bei CES-Nutzenfunktionen den konstanten Wert 1 annimmt, erhalten wir aus der Slutzky-Gleichung in Elastizitätsform nach Ersetzen von $e_{y}$ schließlich mit 


$$
e_{u}=e+\frac{w L}{Y}(\xi-1)
$$

den gesuchten Zusammenhang. Aus Gründen der Übersicht wurde in dieser Darstellung durchweg das Symbol $Y$ anstelle des im Text (und auch sonst) für das maximale Einkommen verwendeten Symbols $Y^{\max }$ $(=w E+r K+T R)$ eingesetzt. 


\section{Anhang 6: Die Implementation eines LES-Nutzenastes}

\section{Die Nachfragefunktionen des Haushalts}

Nach wie vor können die Entscheidungen des privaten Haushalts in zwei Stufen unterteilt werden. Aus dem Optimierungskalkül auf der zweiten Entscheidungsstufe

$$
\begin{array}{cl}
\max _{C_{i}} \quad C=\prod_{i=1}^{14}\left(C_{i}-\gamma_{i}\right)^{\alpha_{i}} & \text { mit } \quad \alpha_{i}>0 ; \sum_{i} \alpha_{i}=1 \\
\text { unter der Nebenbedingung: } & Y^{\text {ver }}=\sum_{i=1}^{14} p_{i} C_{i}
\end{array}
$$

resultieren die Nachfragefunktionen

$$
C_{i}=\gamma_{i}+\frac{\alpha_{i}\left(Y^{v e r}-\Gamma\right)}{p_{i}} \quad \text { mit } \quad \Gamma=\sum_{i} p_{i} \gamma_{i}
$$

Setzen wir die Nachfragefunktionen $C_{i}$ in die direkte Nutzenfunktion ein, so erhalten wir mit

$$
V=\left(Y^{v e r}-\Gamma\right) \prod_{i=1}^{14}\left(\frac{\alpha_{i}}{p_{i}}\right)^{\alpha_{i}}
$$

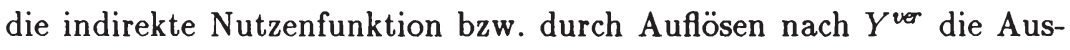
gabenfunktion

$$
Y^{\text {ver }}=V \prod_{i=1}^{14}\left(\frac{p_{i}}{\alpha_{i}}\right)^{\alpha_{i}}+\Gamma .
$$

Diese Gleichung zeigt, daß es keinen idealen Preisindex für die übergeordnete Entscheidungsstufe gibt, der multipliziert mit dem Wert des Nutzenindex $V$ das verfügbare Einkommen ergibt. 
Subtrahiert man jedoch $\Gamma$ vom verfügbaren Einkommen, so erhält man eine homothetische Beziehung zwischen dem diskretionären verfügbaren Einkommen $Y_{d}^{\text {ver }}:=Y^{\text {ver }}-\Gamma$ und dem Nutzenindex $V$ aus diskretionärem Konsum. Aus diesem Grund wird der ursprüngliche CobbDouglas Preisindex $p$ beibehalten.

\section{Die Berechnung der Äquivalenten Variation}

Für die Berechnung der Äquivalenten Variation muß jedoch berücksichtigt werden, daß ein verändertes Preissystem auch die Mindestausgaben $\Gamma$ beeinflußt. Aus einer analog zu der in Anhang 4 geschilderten Vorgehensweise erhalten wir für das LES-System die Berechnungsformel:

$$
E V=\frac{V_{1}-V_{0}}{V_{0}}\left(Y_{0}^{\max }-\Gamma_{0}\right),
$$

wobei die Indizes 0,1 wiederum die Referenz- bzw. die neue Situation kennzeichnen.

\section{Kalibrierung}

Im Gegensatz zu Cobb-Douglas-Nachfragefunktionen können die Parameter des LES-Systems nicht aus einer einzigen Beobachtung modellkonform kalibriert werden. Sind jedoch die Mindestmengen $\gamma_{i}$ bekannt, so sind die diskretionären Ausgabenanteile $\alpha_{i}$ über

$$
\alpha_{i}=\frac{p_{i}\left(C_{i}-\gamma_{i}\right)}{Y^{v}-\Gamma}
$$

aus der Datenbasis ableitbar.

Allerdings ist es schwierig auch nur einigermaßen verläßliche Schätzungen für die Mindestmengen in unserer Abgrenzung der Gütergruppen zu finden ${ }^{147}$. Die meisten Schätzungen von LES-Funktionen - so auch KAISER/SPAHN [1989] - verwenden (sinnvollerweise) Daten aus der

${ }^{147}$ Dies ist auch der Grund, warum das LES-System nicht für das Standardmodell verwendet wurde. 
Einkommens- und Verbrauchsstichprobe, die nach völlig anderen Gesichtspunkten abgegrenzt werden als die Daten der hier verwendeten Input-Output-Tabelle. Eine zusammen mit den Input-Output-Tabellen publizierte Überleitungsmatrix bietet jedoch die Möglichkeit, beide Statistiken vergleichbarer zu machen ${ }^{148}$. In Tabelle 24 sind die Mindestmengen sowie die hinzukalibrierten diskretionären Ausgabenanteile aufgelistet.

Tabelle 24: Mindestkonsummengen und Ausgabenanteile für das LES-System

\begin{tabular}{|c|c|c|}
\hline Gut & $\gamma_{\boldsymbol{i}}$ & $\alpha_{\boldsymbol{i}}$ \\
\hline 1 & 85 & 0.0166 \\
2 & 75 & 0.0373 \\
3 & 150 & 0.0672 \\
4 & 125 & 0.0335 \\
5 & 200 & 0.0636 \\
6 & 210 & 0.0590 \\
7 & 50 & 0.0337 \\
8 & 175 & 0.0531 \\
9 & 450 & 0.1243 \\
10 & 70 & 0.0301 \\
11 & 420 & 0.1766 \\
12 & 110 & 0.0461 \\
13 & 100 & 0.0516 \\
14 & 750 & 0.2075 \\
\hline
\end{tabular}

Die hier unterstellten Mindestmengen orientieren sich an den in KaISER/Wiegard/ZimmermanN ([1990], S. 84) ausgewiesenen Verhältniszahlen und einer entsprechend unserer Güterabgrenzung zusammengefaßten Überleitungsmatrix.

148 Siehe STBA [1988b], S. 201. 


\section{Literaturverzeichnis}

Ahlheim, M. und M. Rose [1984]: Alte und neue Maße individueller Steuerlasten, in: Finanzarchiv N. F. Bd. 42, S. 274-349.

Albers, W. ; K. E. Born; E. Dürr; H. Hesse; A. Kraft; H. Lampert; K. Rose; H.-H. Rupp; H. Scherf; K. Schmidt und W. WitTManN [1980]: Handwörterbuch der Wirtschaftswissenschaft (HdWW) Bd. 8; Stuttgart und New York, Tübingen, Göttingen und Zürich.

Andreae, C.-A. [1980]: Grundsteuern, in: F. Neumark; N. Andel und H. Haller (Hrsg.): HdF Bd. II, 3. Auflage; Tübingen, S. 576608 .

Armington, P. S. [1969]: A Theory of Demand for Products Distinguished by Place of Production, IMF Staff Papers (XVI), Washington, D. C., S. 156-167.

Atkinson, A. B. und J. E. Stiglitz [1972]: The Structure of Indirect Taxation and Economic Efficiency, in: Journal of Public Economics Vol. 1, S. 97-120.

Atkinson, A. B. und J. E. Stiglitz [1980]: Lectures on Public Economics; London u. a. O.

Auerbach, A. J. und M. Feldstein [1985]: Handbook of Public Economics Vol. 1; Amsterdam u. a. O.

Auerbach, A. und L. Kotlikoff [1987]: Dynamic Fiscal Policy, Cambridge University Press; Cambridge u. a. O.

Balassa, B. [1965]: Tariff Protection in Industrial Countries: An Evaluation, in: Journal of Political Economy Bd. 73, S. 573-594; wiederveröffentlicht in: R. E. CAves und H. G. Johnson (Hrsg.) [1968]: Readings in International Economics; Homewood, Illinois.

Ballard, C. und L. Goulder [1985]: Consumption Taxes, Foresight, and Welfare: A Computable General Equilibrium Analysis, 
in: J. Piggott und J. Whalley (Hrsg.): New Developments in Applied General Equilibrium Analysis, Cambridge University Press; Cambridge u. a. O.

Ballard, C. und J. B. Shoven [1987]: The Value Added Tax: The Efficiency Cost of Achieving Progressivity by Using Exemptions, in: M. J. Boskin (ed.): Modern Developments in Public Finance; New York, S. 109-129.

Ballard, C. ; J. B. Shoven und J. Whalley [1982]: The Welfare Costs of Distortions in the United States Tax System: A General Equilibrium Approach, in: NBER Working Paper Nr. 1043; Cambridge, Massachusetts.

Ballard, C. ; J. K. Scholz and J. B. Shoven [1987]: The Value Added Tax: A General Equilibrium Look at its Efficiency and Incidence, in: M. Feldstein (Hrsg.): The Effects of Taxation and Capital Accumulation; Chicago und London, S. 445-474.

Ballard, C. L. ; D. Fullerton; J. B. Shoven und J. Whalley [1985]: A General Equilibrium Model for Tax Policy Evaluation, National Bureau of Economic Research Monograph, Chicago und London.

Bardazzi, R. ; M. Grassini und E. Langobardi [1989]: ValueAdded Taxes and Indirect Taxes in an EEC Country Model: The Italian Case, unveröffentlichtes Manuskript; Florenz.

Barham, V. ; S. N. Poddar and J. Whalley [1987]: The Tax Treatment of Insurance Under a Consumption Type, Destination Basis VAT, in: National Tax Journal Vol. 40, Columbus, Ohio, S. 171-182.

BEA, F. X. [1980]: Umsatzsteuern. Ziele und Ausgestaltung, in: W. Albers; K. E. Born u. a.: HdWW Bd. 8; Stuttgart u. a. O., S. $27-40$.

Bedau, K.-D. und G. Göseke [1972]: Wirken indirekte Steuern regressiv? Die Belastung der privaten Haushalte mit indirekten Steuern, in: DIW-Wochenbericht 39/21, S. 187-194.

Bhatia, K. B. [1982]: Value-Added Tax and the Theory of Tax Inci- 
dence, in: Journal of Public Economics Bd. 19, S. 203-223.

Bhatia, K. B. [1986]: Taxes, Intermediate Goods, and Relative Prices, in: Journal of Public Economics Bd. 31; London, Kanada, S. 197-213.

Blackorby, C. ; G. Lady; D. Nissen und R. R. Russel [1970]: Homothetic Separability and Consumer Budgeting, Econometrica Vol. 38, S. 468-472.

Boadway, R. und J. Treddenick [1978]: A General Equilibrium Computation of the Effects of the Canadian Tariff Structure, in: The Canadian Journal of Economics Vol. 11, S. 424-446.

Bös, D. ; M. Rose und C. SEIDL [1988]: Welfare and Efficiency in Public Economics; Berlin und Heidelberg.

Bohley, P. und G. Tolkemitt [1979]: Wirtschaftswissenschaft als Grundlage staatlichen Handelns. Heinz Haller zum 65. Geburtstag; Tübingen.

Boskin, M. J. [1987]: Modern Developments in Public Finance, New York.

Bronfenbrenner, M. [1950]: The Japanese Value-Added Sales Tax, in: National Tax Journal Vol. 3; New York, S. 298-313.

BRÜMMERHoFF, D. [1988]: Finanzwissenschaft, 3., völlig überarbeitete und erweiterte Auflage; München und Wien.

Brunner, K. J. und H.-G. Petersen [1990]: Simulation Models in Tax and Transfer Policy. Proceedings of an International Symposium; Frankfurt und New York.

CADDY, V. [1976]: Empirical Estimation of the Elasticity of Substitution: A Review, Mimeo - Industries Assistance Commission; Melbourne.

Cnossen, S. [1977]: Excise Systems. A Global Study of the Selective Taxation of Goods and Services, The John Hopkins University Press; Baltimore and London.

Cnossen, S. [1981]: The Netherlands, in: H. J. Aaron (Hrsg.): The Value Added Tax: Lessons from Europe, The Brookings Institu- 
tion; Washington, D. C., S. 43-59.

Corden, W. M. [1966]: The Structure of a Tariff System and the Effective Protective Rate, Journal of Political Economy Bd. 74, S. 221-237.

Cornwall, R. R. [1984]: Introduction to the Use of General Equilibrium Analysis; Amsterdam u. a. O.

Costede, J. (Hrsg. für) Vereinigung zur wissenschaftlichen Pflege des Umsatzsteuerrechts e. V. [1985]: Umsatzsteuerkongreß-Bericht 1985; Köln.

Davies, D. G. [1986]: United States Taxes and Tax Policy, Cambridge University Press; Cambridge u. a. O.

Deaton, A. [1974]: A Reconsideration of the Empirical Implications of Additiv Preferences, in: Journal of Public Economics Vol. 8, S. 338-348.

Deaton, A. [1977]: Equity, Efficiency and the Structure of Indirect Taxation, in: Journal of Public Economics Vol. 8, S. 299-312.

Deaton, A. [1979]: Optimally Uniform Commodity Taxes, in: Economics Letters Vol 2, S. 367-371.

Deaton, A. [1981]: Optimal Taxes and the Structure of Preferences, in: Econometrica Vol. 49, S. 1245-1260.

Deaton, A. und J. Muellbauer [1980]: Economics and Consumer Behavior, Cambridge University Press; Cambridge u. a. O.

Diewert, E. W. [1985]: The Measurement of Waste and Welfare in Applied Equilibrium Models, in: J. PiggotT und J. WhalLEY (Hrsg.): New Developments in Applied Equilibrium Analysis, Cambridge University Press; Cambridge u. a. O.

Dorow, F. [1972]: Zur Behandlung der unterstellten Bankgebühr in den Volkswirtschaftlichen Gesamtrechnungen. In: Wirtschaft und Statistik Bd. 7/72; Stuttgart und Mainz, S. 377-382.

Due, J. F. [1988]: Indirect Taxation in Developing Economies, Revised Edition, The John Hopkins University Press; Baltimore and London. 
Due, J. F. und J. L. Mikesell [1984]: Sales Taxation. State and Local Structure and Administration, second printing, The John Hopkins University Press; Baltimore and London.

Dennerlein, R. [1982]: Die Belastungswirkung der indirekten Steuern in der Bundesrepublik; Berlin.

Dziadkowski, D. [1986]: Umsatzsteuer, 2. Auflage; München, Wien.

Ebel, R. D. [1972]: The Michigan Business Activities Tax. ValueAdded Taxation in the Subnational Economy, MSU Business Studies; East Lansing, Michigan.

Ebert, U. [1988]: On the Evaluation of Tax Systems, in: D. Bös; M. Rose und C. Seidl (Hrsg.): Welfare and Effiency in Public Economics; Berlin u. a. O., S. 263-280.

European Parliament [1989]: Economic Problems of Value-Added Tax Harmonization and Completition of the International Market, Research and Documentation Papers, Economic Series No. 15; Luxembourg.

Fehr, H. ; C. Rosenberg und W. Wiegard [1990]: Value-Added Tax Harmonisation in the EEC: Some (Preliminary) Applied General Equilibrium Calculations, International Institute of Public Finance, 46th Congress; Brüssel.

Feldstein, M. [1983]: Behavioral Simulation Methods in Tax Policy Analysis, Chicago University Press; Chicago.

Feldstein, M. [1987]: The Effects of Taxation and Capital Accumulation, Chicago and London.

Fellner, W. J. [1967]: Ten Economic Studies in the Tradition of Irving Fisher; New York.

Fischer, L. [1983]: Unternehmung und Steuer. Festschrift zur Vollendung des 80. Lebensjahres von Peter Scherpf; Wiesbaden.

Fullerton, D. ; J. B. Shoven und J. Whalley [1983]: Replacing the U. S. Income Tax with a Progressive Consumption Tax: A Sequenced General Equilibrium Approach, in: Journal of Public Economics Vol. 20, S. 3-23. 
Fullerton, D. ; A. T. King; J. B. Shoven und J. Whalley [1981]: Tax Integration in the U. S.: A General Equilibrium Approach, in: American Economic Review Vol. 71, S. 677-691.

Garber, H. D. und D. G. Raboy [1990]: Value-Added Taxation of Financial Services, in: M. L. Weidenbaum, D. G. Raboy und E. S. Christian, JR. (Hrsg.): The Value-Added Tax: Orthodoxy and New Thinking; Boston, Dordrecht, London, S.163-187.

Gerloff, W. und F. Meisel [1927]: Handbuch der Finanzwissenschaft ( $\mathrm{HdF}) \mathrm{Bd}$. II, 1. Auflage; Tübingen.

Gillis, M. [1990]: The VAT and Financial Services, in: M. Gillis, C. S. Shoup und G. P. Sicat (Hrsg.): Value Added Taxation in Developing Countries; Washington, D. C., S. 83-94.

Gillis, M. , C. S. Shoup und G. P. Sicat [1990]: Value Added Taxation in Developing Countries, A World Bank Symposium, The World Bank; Washington, D. C.

Goergen, R. [1985]: Kritische Bestandsaufnahme des Entwicklungsstandes der Umsatzsteuerharmonisierung in der EWG, in: J. Costede (Hrsg. für) Vereinigung zur wissenschaftlichen Pflege des Umsatzsteuerrechts e. V.: Umsatzsteuerkongreß-Bericht 1985; Köln, S. 15-32.

Gorman, W. M. [1957]: Tariffs, Retaliation, and the Elasticity of Demand for Imports, in: Review of Economic Studies No. 25, S. 133-162.

Gottfried, P. und W. Wiegard [1990]: Exemption versus Zero Rating: A Hidden Problem of VAT, Regensburger Diskussionsbeiträge Nr. 219.

Gottfried, P. ; E. Stöss und W. Wiegard [1990]: Applied General Equilibrium Tax Models: Prospects, Examples, Limits, in: K. J. Brunner und H.-G. Petersen (Hrsg.): Simulation Models in Tax and Transfer Policy. Proceedings of an International Symposium; Frankfurt und New York, S. 205-244.

Goulder, L. und L. Summers [1987]: Tax Policy, Asset Prices, and Growth: A General Equilibrium Analysis, NBER Working Paper 
No. 2128, National Bureau of Economic Research; Cambridge.

Goulder, L. ; J. B. Shoven und J. Whalley [1983]: Domestic Tax Policy and the Foreign Sector: The Importance of Alternative Foreign Sector Formulations to Results from a General Equilibrium Tax Analysis Model, in: M. Feldstein (Hrsg.): Behavioral Simulation Methods in Tax Policy Analysis, Chicago University Press; Chicago, S. 333-364.

Grabower, R.; D. Herting und G. Schwarz [1962]: Die Umsatzsteuer. Ihre Geschichte und gegenwärtige Ausgestaltung in In- und Ausland; Köln u. a. O.

Gutting, B. [1987]: Der Einfluß der Gemeindesteuern auf die Finanzierungs- und Investitionsentscheidung von Unternehmen, in: Finanzarchiv N. F. Bd. 45, S. 25-44.

Harberger, A. C. [1962]: The Incidence of the Corporate Income Tax, in: Journal of Public Economics. Wiederveröffentlicht In: Harberger, A. C. (Hrsg.) [1974]: Taxation and Welfare; Boston, S. $135-162$.

Harris, R. G. und J. G. MacKinnon [1979]: Computing Optimal Tax Equilibria, in: Journal of Public Economics Vol. 11, S. 197212.

Hatta, T. [1986]: Welfare Effects of Changing Commodity Tax Rates Toward Uniformity, in: Journal of Public Economics Vol. 29, S. 99112.

Hausmann, J. A. [1985]: Taxes and Labor Supply, in: A. J. AuerBACH und M. FELdSTEIN (Hrsg.): Handbook of Public Economics Vol. 1; Amsterdam u. a. O., S. 213-263.

Helmstädter, E.; B. Meyer; E. Kleine und J. Richtering [1983]: Die Input-Output-Analyse als Instrument der Strukturforschung; Tübingen.

Hemming, R. and J. A. KaY [1981]: The United Kingdom, in: H. J. Aaron (Hrsg.): The Value Added Tax: Lessons from Europe, The Brookings Institution, Washington, D. C. 
Henderson, J. M. und R. E. QUandt [1983]: Mikroökonomische Theorie. Eine mathematische Darstellung, 5., überarbeitete Auflage; München.

Heubes, J. [1969]: Sektorale Produktionsfunktionen der Deutschen Industrie; Bonn.

Hirte, G. und W. Wiegard [1988]: An Introduction to Applied General Equilibrium Tax Modelling, in: D. Bös; M. Rose und C. SEIDL (Hrsg.): Welfare and Efficiency in Public Economics; Berlin und Heidelberg, S. 167-203.

Holub, H.-W. und H. Schnabl [1985]: Input-Output-Rechnung: Input-Output-Tabellen. 2. Auflage; München und Wien.

ITo, H. [1955]: Theorie und Technik der Nettoumsatzsteuer in Japan, in: Finanzarchiv N. F. Bd 15; Tübingen, S. 447-478.

Johnson, H. G. [1953]: Optimum Tariffs and Retaliation, in: Review of Economic Studies No. 21, S. 142-153.

Johnson, H. G. [1965]: The Theory of Tariff Structure with Special Reference to World Trade and Development, in: H. G. JoHNSON and P. B. Kenen (Hrsg.): Trade and Development, Institut Universitaire des Hautes Etudes Internationales; Genf.

Jorgenson, D. W. [1973]: Technology and Decision Rules in the Theory of Investment Behavior, in: Quarterly Journal of Economics Bd. 87, S.523-543.

Jorgensen, D. W. [1984]: Econometric Methods for Applied General Equilibrium Analysis, in: H. E. SCARF und J. B. SHoven (Hrsg.): Applied General Equilibrium Analysis, Cambridge University Press; Cambridge u. a. $O$.

Jorgenson, D. W. und D. Slesnick [1985]: General Equilibrium Analysis of Economic Policy, in: J. Piggott und J. Whalley (Hrsg.): New Developments in Applied General Equilibrium Analysis, Cambridge University Press; Cambridge u. a. O., S. 293-370.

KaISER, H. [1989]: Die Mehrwertsteuerbelastung privater Haushalte in der Bundesrepublik Deutschland. Ein Vergleich empirischer 
Inzidenz analysen auf Basis der Einkommens- und Verbrauchsstichproben 1969,1973 und 1978, in: DIW Vierteljahreshefte zur Wirtschaftsforschung, Jahrgang 1989, S. 24-44.

Kaiser, H. und P. B. Spahn [1989]: On the Efficiency and Distributive Justice of Consumption Taxes: A Study on VAT in West Germany, in: Journal of Economics Vol. 49, S. 199-218.

Kaiser, H. ; W. Wiegard und H. G. Zimmermann [1990]: Testing the Reliability of Optimal Tax Calculations, in: Finanzarchiv N. F. Bd. 48, S. 77-96.

KaY, J. A. and N. A. WARren [1980]: Effective Rates of Value Added Tax, Working Paper No. 16, Institute for Fiscal Studies; London.

Kenoe, T. J. [1980]: An Index Theorem for General Equilibrium Models with Production, in: Econometrica Vol. 78, S. 1211-1232

Kenoe, T. J. [1984]: Computing all of the Equilibria of Economies with Two Factors of Production, in: Journal of Mathematical Economics Vol. 13, S. 207-223.

Kenoe, T. J. [1985a]: The Comparative Statics Properties of Tax Models, in: Canadian Journal of Economics Vol. 18, S. 314-334.

KеноE, T. J. [1985b]: A Numerical Investigation of Multiplicity of Equilibria, in: A. S. ManNE (Hrsg.): Economic Equilibrium: Model Formulation and Solution. Mathematical Programming Study 23; Amsterdam u. a. O., S. 240-258.

Kehoe, T. ; P. J. Noyola; A. Manresa; C. Polo und F. Sancho [1988]: A General Equilibrium Analysis of the 1986 Tax Reform in Spain, in: European Economic Review Bd. 32, S.334-342.

Kehoe, T. J. und J. Whalley [1985]: Uniqueness of Equilibrium in Large-Scale Numerical General Equilibrium Models, in: Journal of Public Economics Vol. 28, S. 247-255.

Keller, W. J. [1976]: A Nested CES-Type Utility Function and its Demand and Price-Index Functions, in: European Economic Review Vol. 7, S. 175-186. 
Killingsworth, M. R. [1983]: Labour Supply, Cambridge University Press; Cambridge u. a. O.

KitTerer, W. [1978]: Die Belastung der privaten Haushalte mit indirekten Steuern, in: RWI-Mitteilungen, 29. Jahrgang; Berlin.

KitTerer, W. [1986]: Effizienz und Verteilungswirkungen des Steuersystems; Frankfurt am Main, Bern, New York.

Kitterer, W. und J. Fronia [1981]: Belastungswirkungen der Umsatzsteuer. Gutachten im Auftrag des Bundesministers für Wirtschaft, Forschungsberichte Serie A, Nr. 33, Institut für Angewandte Wirtschaftsforschung; Tübingen.

Kopsch, G. [1983]: Der Staat in den Volkswirtschaftlichen Gesamtrechnungen 1960 bis 1982, in: Wirtschaft und Statistik Bd. 8/83; Stuttgart und Mainz, S. 749-769.

Koutsoyannis, A. [1981]: Theory of Econometrics, 2. Auflage; London.

KromphardT, J. [1977]: Wachstum und Konjunktur. Grundlage ihrer theoretischen Analyse und wirtschaftspolitischen Steuerung, 2., neubearbeitete Auflage; Göttingen.

KüHN, B. [1988]: Die geplante Einkommensteuerreform 1990. Simulationsergebnisse eines Empirischen Allgemeinen Gleichgewichtsmodells; Frankfurt a. Main u.a. O.

LaU, L. J. [1984]: Comments, in: H. E. Scarf und J. B. Shoven (Hrsg.): Applied General Equilibrium Analysis, Cambridge University Press; Cambridge u. a. O., S. 127-137.

LisT, H. [1983]: Der Systemwandel im Umsatzsteuerrecht, in: Vereinigung zur wissenschaftlichen Pflege des Umsatzsteuerrechts e. V. (hrsg. durch) C. LoHSE und W. SchöLl: UmsatzsteuerkongreßBericht 1982/83; Köln, S. 7-23.

Lindholm, R. W. [1970]: Value Added Tax and other Tax Reforms, Chicago.

Lindholm, R. W. [1980]: The Economics of VAT; Lexington, Massachusetts, Toronto. 
LitTmann, K. [1980]: Überblick über die Ertragsteuern, in: F. NEUMark; N. ANDel und H. Haller (Hrsg.): HdF Bd. II, 3. Auflage; Tübingen, S. 565-575.

LitTman, K. [1980]: Gewerbesteuern, in: F. Neumark; N. ANDEL und H. Haller (Hrsg.): HdF Bd. II, 3. Auflage; Tübingen, S. 609630 .

LohsE, C. und W. SchöLl (Hrsg. für) Vereinigung zur wissenschaftlichen Pflege des Umsatzsteuerrechts e. V. [1983]: Umsatzsteuerkongreß-Bericht 1982/83; Köln.

LuCAS, R. [1967]: Adjustment Costs and the Theory of Supply, Journal of Political Economy Vol. 75, S. 321-334.

Manne, A. S. [1985]: Economic Equilibrium: Model Formulation and Solution. Mathematical Programming Study 23; Amsterdam u. a. O.

Mansur, A. und J. Whalley [1984]: Numerical Specification of Applied General Equilibrium Models: Estimation, Calibration, and Data, in: H. E. Scarf und J. B. Shoven (Hrsg.): Applied General Equilibrium Analysis, Cambridge University Press; Cambridge u. a. O., S. 69-127.

McLure, C. E. JR. [1968]: Merit Wants. A Normativ Empty Box, in: Finanzarchiv N. F. Bd. 27; Tübingen, S. 474-483.

McLure, C. E. JR. [1987]: The Value-Added Tax. Key to Deficit Reduction?, American Enterprise Institute for Public Policy Research; Washington, D. C.

McLure, C. E. JR. [1987a]: VAT, Income Distribution, and Tax Incidence, World Bank Working Paper.

McLure, C. E. Jr. und N. B. Ture [1972]: Value-Added Tax: Two Views, American Enterprise Institute for Policy Research; Washington, D. C.

McLure, C. E. JR. und R. T. WAyne [1975]: A Simplified Exposition of the Harberger Modell, I: Tax Incidence, in: National Tax Journal Vol. 28, S. 1-28. 
Merrill, O. H. [1972]: Applications and Extensions of an Algorithm that Computes Fixed Points of Certain Upper Semi-Continuous Point to Set Mappings, Ph.D. Dis., University of Michigan.

Metze, I. [1980]: Kapitalertragsteuer, in: F. Neumark; N. ANDEL und H. Haller (Hrsg.): HdF Bd. II, 3. Auflage; Tübingen, S. 633643 .

Musgrave, R. A. und P. B. Musgrave [1973]: Public Finance in Theory and Practice; New York u. a. O.

Musgrave, R. A. ; K. E. Case und H. Leonard [1974]: The Distribution of Fiscal Burdens and Benefits, in: Public Finance Quarterly $2 / 3$, S.259-311.

Neue Wirtschafts-Briefe [1984]: Wichtige Steuergesetze mit Durchführungsverordnung, 31. geänderte Auflage; Herne und Berlin.

NEUMARK, F. [1970]: Grundsätze gerechter und ökonomisch rationaler Steuerpolitik; Tübingen.

Neumark, F. ; N. Andel und H. Haller [1980]: Handbuch der Finanzwissenschaft (HdF) Bd. II, 3., gänzlich neubearbeitete Auflage; Tübingen.

Organisation for Economic Co-Operation and Development (OECD) [1988]: Taxing Consumption, Paris.

Pechman, J. A. and B. A. Okner [1974]: Who Bears the Tax Burden?, The Brookings Institution; Washington, D. C.

Pechman, J. A. [1985]: Who Paid the Taxes 1966-1985? The Brookings Institution; Washington, D. C.

Pereira, A. M. [1988]: DAGEM - A Dynamic Applied General Equilibrium Model for Tax Policy Evaluation, San Diego Working Paper No. 88-17, University of California; San Diego.

PhiLIPowski, R. [1985]: Umsatzsteuer: Verbrauch- oder Verkehrsteuer?, in: Vereinigung zur wissenschaftlichen Pflege des Umsatzsteuerrechts e. V. (hrsg. durch J. CosTEDE: UmsatzsteuerkongreB-Bericht 1985; Köln, S. 183-207. 
Piggote, J. und J. Whalley [1985]: UK Tax Policy and Applied General Equilibrium Analysis, Cambridge University Press; Cambridge u. a. O.

Pigott, J. und J. Whalley [1985]: New Developments in Applied General Equilibrium Analysis, Cambridge University Press; Cambridge u. a. O.

Pohmer, D. [1979]: Zum Grenzausgleich bei der Umsatzsteuer, in: P. Bohley und G. Tolkemitt (Hrsg.): Wirtschaftswissenschaft als Grundlage staatlichen Handelns; Tübingen, S.249-280.

Pohmer, D. [1980]: Allgemeine Umsatzsteuern, in: F. Neumark; N. Andel und H. Haller (Hrsg.): HdF Bd. II, 3. Auflage; Tübingen, S. 647-707.

Pohmer, D. [1983]: Zur Systematik und Praxis der Mehrwertsteuern unter besonderer Berücksichtigung der Entwicklung in Europa, in: L. Fischer (Hrsg.): Unternehmung und Steuer. Festschrift zur Vollendung des 80. Lebensjahres von Peter Scherpf; Wiesbaden, S. 375-395.

Popitz, J. [1927]: Allgemeine Verbrauchsteuer, in: W. Gerloff und F. Meisel (Hrsg.): HdF Bd. II; Tübingen, S. 180-197.

RABoY, D. G. [1990]: Preferential Treatment: The Implications for Horizontal Equity among Companies, in: M. L. WeIdenbaum, D. G. Raboy und E. S. Christian, Jr. (Hrsg.): The ValueAdded Tax: Orthodoxy and New Thinking; Boston, Dordrecht, London, S. 87-102.

Reich, U. P. ; C. Stahmer u. a. [1984]: Darstellungskonzepte der Input-Output-Rechnung; Stuttgart und Mainz.

Rose, G. [1987]: Betrieb und Steuer, 2. Buch: Die Verkehrsteuern, 8. Auflage; Wiesbaden.

Rose, K. [1981]: Theorie der Außenwirtschaft, 8., überarbeitete Auflage; München.

Roskamp, K. W. [1976]: Labor Productivity and the Elasticity of Factor Substitution in West German Industries 1950-1960, in: The 
Review of Economics and Statistics, S. 366-371.

Scarf, H. [1967]: On the Computation of Equilibrium Prices, in W.

J. Fellner (Hrsg.): Ten Economic Studies in the Tradition of Irving Fisher; New York, S. 207-230.

Scarf, H. [1973]: The Computation of Economic Equilibria; New Haven und London.

Scarf, H. E. und J. B. Shoven [1984]: Applied General Equilibrium Analysis, Cambridge University Press; Cambridge u. a. O.

Schneider, D. [1980]: Körperschaftsteuer, in: F. Neumark; N. ANDel und H. Haller (Hrsg.): HdF Bd. II, 3. Auflage; Tübingen, S. 509-564.

Shoven, J. B. und J. Whalley [1977]: Equal Yield Tax Alternatives. General Equilibrium Computational Techniques, in: Journal of Public Economics Vol. 8, S. 211-224.

Siemens, W. von [1919]: Veredelte Umsatzsteuer; Siemensstadt. Nach dem Tode des Verfassers wurde von C. F. von Siemens 1921 eine zweite, erweiterte Auflage herausgegeben.

SinN, H.-W. [1985]: Kapitaleinkommensbesteuerung. Eine Analyse der intertemporalen, internationalen und intersektoralen Allokationswirkungen; Tübingen.

Skolka, J. [1987]: Zurechenbarer Steuergehalt der Endnachfrage. Eine Input-Output-Untersuchung für Österreich, in: WiFo-Monatsberichte, 60. Jahrgang; Wien, S.632-649.

St-Hilaire, F. und J. Whalley [1983]: A Microconsistent Equilibrium Data Set for Canada for Use in Tax Policy Analysis, in: Review of Income and Wealth Vol. 29, S. 1007-1051.

St-Hillaire, F. und J. Whalley [1987]: A Microconsistent Data Set for Canada for Use in Regional General Equilibrium Policy Analysis, in: Review of Income and Wealth Vol 33, S. 327-346.

Stahmer, C. [1983]: Input-Output-Rechnung des statistischen Bundesamtes, in: Wirtschaft und Statistik Bd. 8/83, Stuttgart und Mainz, S. 601-609. 
STAHMER, C. [1984]: Überlegungen zu einem System von Input-Output-Tabellen für die Bundesrepublik Deutschland, in: U. P. Reich, C. Stahmer u. a. (Hrsg.): Darstellungskonzepte der Input-Output-Rechnung; Stuttgart und Mainz, S. 41-72.

Statistisches Bundesamt (StBA) [1982]: Systematische Verzeichnisse: Systematik der Wirtschaftszweige mit Betriebs- und ähnlichen Benennungen, Ausgabe 1979; Stuttgart und Mainz.

Statistisches Bundesamt (StBA) [1985a]: Fachserie 18: Volkswirtschaftliche Gesamtrechnungen, Reihe 1: Konten und Standardtabellen 1984; Stuttgart und Mainz.

Statistisches Bundesamt (StBA) [1985b]: Fachserie 18: Volkswirtschaftliche Gesamtrechnungen, Reihe S.8: Revidierte Ergebnisse 1960 bis 1984; Stuttgart und Mainz.

Statistisches Bundesamt (StBA) [1986a]: Fachserie 14: Finanzen und Steuern, Reihe 8: Umsatzsteuer 1984; Stuttgart und Mainz.

Statistisches Bundesamt (StBA) [1986b]: Fachserie 14: Finanzen und Steuern, Reihe 10.1: Realsteuervergleich 1985; Stuttgart und Mainz.

Statistisches Bundesamt (StBA) [1987a]: Statistisches Jahrbuch 1986; Stuttgart und Mainz.

Statistisches Bundesamt (StBA) [1987b]: Fachserie 18: Volkswirtschaftliche Gesamtrechnungen, Reihe S. 10: Der Staat in der Volkswirtschaftlichen Gesamtrechnung 1950-1986; Stuttgart und Mainz.

Statistisches Bundesamt (STBA) [1988a]: Fachserie 18: Volkswirtschaftliche Gesamtrechnungen, Reihe 1: Konten und Standardtabellen 1987 Hauptbericht; Stuttgart und Mainz.

Statistisches Bundesamt (StBA) [1988b]: Fachserie 18: Volkswirtschaftliche Gesamtrechnungen, Reihe 2: Input-Output-Tabellen 1984; Stuttgart und Mainz.

Statistisches Bundesamt (StBA) [1989]: Fachserie 18: Volkswirtschaftliche Gesamtrechnungen, Reihe 1.3: Konten und Standardta- 
bellen 1988, Hauptbericht; Wiesbaden.

STEPPERT, H [1990]: Die Vorschläge der EG-Kommission zur UStHarmonisierung - ein Weg zur Verwirklichung des Binnenmarktes? in: Umsatzsteuer-Rundschau Bd. 9, S. 269-271.

Stern, R. M. ; J. Francis und B. Schumacher [1976]: Price Elasticities in International Trade. An Annotated Bibliography; London.

Stiglitz, J. E. [1973]: Taxation, Corporate Financial Policy, and the Cost of Capital, in: Journal of Public Economics Vol. 2, S. 1-34.

Stiglitz, J. E. und B. Schönfelder [1989]: Finanzwissenschaft, 2. Auflage; München und Wien.

Strotz, R. H. [1957]: The Empirical Implications of a Utility Tree, in: Econometrica Vol. 25, S. 269-280.

Strotz, R. H. [1959]: The Utility Tree - A Correction and Further Appraisal, in: Econometrica Vol. 27, S. 482-488.

Sullivan, C. K. [1965]: The Tax On Value Added, Columbia University Press; New York and London.

TAIT, A. A. [1988]: Value-Added Tax. International Practice and Problems, International Monetary Fund; Washington, D. C.

Takayama, A. [1986]: Mathematical Economics, 2. Auflage, Cambridge University Press; Camdridge o. a. O.

VARIAN, H. R. [1985]: Mikroökonomie, 2., völlig überarbeitete und stark erweiterte Auflage; München und Wien.

Weidenbaum, M. L. ; D. G. Raboy und E. S. Christian, Jr. [1990]: The Value-Added Tax: Orthodoxy and New Thinking, 2nd ed., Center for the Study of American Business; Boston, Dordrecht, London.

Wetzler, J. W. [1979]: The Role of a Value Added Tax in Financing Social Security, in: National Tax Journal, Vol. 32; Cambridge, Massachusetts, S. 334-345.

Wiegard, W. [1985]: Die Algorithmen von Scarf und Merrill zur 
numerischen Berechnung Allgemeiner Gleichgewichte. Eine Einführung mit Beispielen aus der Steuerpolitik, in: Zeitschrift für Wirtschafts- und Sozialwissenschaften Bd. 105, S. 709-741.

Wissenschaftlicher Beirat beim Bundesministerium der FiNANZEN [1982]: Gutachten zur Reform der Gemeindesteuern in der Bundesrepublik Deutschland, Schriftenreihe des Bundesministeriums der Finanzen, Heft 31; Bonn.

ZWER, R. [1981]: Internationale Wirtschafts- und Sozialstatistik; München und Wien. 


\section{FINANZWISSENSCHAFTLICHE SCHRIFTEN}

Band 1 Werner Steden: Finanzpolitik und Einkommensverteilung. Ein Wachstums- und Konjunkturmodell der Bundesrepublik Deutschland. 1979.

Band 2 Rainer Hagemann: Kommunale Finanzplanung im föderativen Staat. 1976.

Band 3 Klaus Scherer: Maßstăbe zur Beurteilung von konjunkturellen Wirkungen des öffentlichen Haushalts. 1977.

Band 4 Brita Steinbach: "Formula Flexibility" - Kritische Analyse und Vergleich mit diskretionärer Konjunkturpolitik. 1977.

Band 5 Hans-Georg Petersen: Personelle Einkommensbesteuenung und Inflation. Eine theoretisch-empirische Analyse der Lohn- und veranlagten Einkommenstever in der Bundesrepublik Deutschland. 1977.

Band 6 Friedemann Tetsch: Raumwirkungen des Finanzsystems der Bundesrepublik Deutschland. Eine Untersuchung der Auswirkungen der Finanzreform von 1969 auf die Einnahmenposition der untergeordneten Gebietskörperschaften und ihrer regionalpolitischen Zieladåquanz. 1978.

Band 7 Wilhelm Pfăhler: Normative Theorie der fiskalischen Besteuerung. Ein methodologischer und theoretischer Beitrag zur Integration der normativen Besteuerungstheorie in der Wohlfahrtstheorie. 1978.

Band 8 Wolfgang Wiegard: Optimale Schattenpreise und Produktionsprogramme für öffentliche Unternehmen. Second-Best Modelle im finanzwirtschaftlichen Staatsbereich. 1978.

Band 9 Hans P. Fischer: Die Finanzierung des Umweltschutzes im Rahmen einer rationalen Umweltpolitik. 1978.

Band 10 Rainer Paulenz: Der Einsatz finanzpolitischer Instrumente in der Forschungs- und Entwicklungspolitik. 1978.

Band 11 Hans-Joachim Hauser: Verteilungswirkungen der Staatsverschuldung. Eine kreislauftheoretische Inzidenzbetrachtung. 1979.

Band 12 Gunnar Schwarting: Kommunale Investitionen. Theoretische und empirische Untersuchungen der Bestimmungsgründe kommunaler Investitionstătigkeit in NordrheinWestfalen 1965-1972. 1979.

Band 13 Hans-Joachim Conrad: Stadt-Umland-Wanderung und Finanzwirtschaft der Kernstädte. Amerikanische Erfahrungen, grundsătzliche Zusammenhănge und eine Fallstudie für das Ballungsgebiet Frankfurt am Main. 1980.

Band 14 Cay Folkers: Vermögensverteilung und staatliche Aktivităt. Zur Theorie distributiver Prozesse im Interventionsstaat. 1981.

Band 15 Helmut Fischer: US-amerikanische Exportförderung durch die DISC-Gesetzgebung. 1981.

Band 16 Günter Ott: Einkommensumverteilungen in der gesetzlichen Krankenversicherung. Eine quantitative Analyse. 1981.

Band 17 Johann Hermann von Oehsen: Optimale Besteuerung. (Optimal Taxation). 1982.

Band 18 Richard Kössler: Sozialversicherungsprinzip und Staatszuschüsse in der gesetzlichen Rentenversicherung. 1982.

Band 19 Hinrich Steffen: Zum Handlungs- und Entscheidungsspielraum der kommunalen Investitionspolitik in der Bundesrepublik Deutschland. 1983.

Band 20 Manfred Scheuer: Wirkungen einer Auslandsverschuldung des Staates bei flexiblen Wechselkursen. 1983. 
Band 21 Christian Schiller: Staatsausgaben und crowding-out-Effekte. Zur Effizienz einer Finanzpolitik keynesianischer Provenienz. 1983.

Band 22 Hannelore Weck: Schattenwirtschaft: Eine Möglichkeit zur Einschränkung der öffentlichen Verwaltung? Eine okonomische Analyse. 1983.

Band 23 Wolfgang Schmitt: Steuern als Mittel der Einkommenspolitik. Eine Ergănzung der Stabilitătspolitik? 1984.

Band 24 Wolfgang Laux: Erhöhung staatswirtschaftlicher Effizienz durch budgetăre Selbstbeschrânkung? Zur Idee einer verfassungsmaßßig verankerten Ausgabengrenze. 1984.

Band 25 Brita Steinbach-van der Veen: Steuerinzidenz. Methodologische Grundlagen und empirisch-statistische Probleme von Lânderstudien. 1985.

Band 26 Albert Peters: Okonomische Kriterien für eine Aufgabenverteilung in der Marktwirtschaft. Eine deskriptive und normative Betrachtung für den Allokationsbereich. 1985.

Band 27 Achim Zeidler: Moglichkeiten zur Fortsetzung der Gemeindefinanzreform. Eine theoretische und empirische Analyse. 1985.

Band 28 Peter Bartsch: Zur Theorie der lângerfristigen Wirkungen 'expansiver' Fiskalpolitik. Eine dynamische Analyse unter besonderer Berücksichtigung der staatlichen Budgetbeschrånkung und ausgewăhlter Möglichkeiten der ôffentlichen Defizittinanzienung. 1986.

Band 29 Konrad Beiwinkel: Wehrgerechtigkeit als finanzpolitisches Verteilungsproblem. Möglichkeiten einer Kompensation von Wehrungerechtigkeit durch monetäre Transfers. 1986.

Band 30 Wolfgang Kitterer: Effizienz- und Verteilungswirkungen des Steuersystems. 1986.

Band 31 Heinz Dieter Hessler: Theorie und Politik der Personalsteuern. Eine Kritik ihrer Einkommens- und Vermogensbegriffe. 1987.

Band 32 Wolfgang Scherf: Die beschäftigungspolitische und fiskalische Problematik der Arbeitgeberbeitrăge zur Rentenversicherung. Eine Auseinandersetzung mit der Kritik an der lohnbezogenen Beitragsbemessung. 1987.

Band 33 Andreas Măstle: Die Steuenunion. Probleme der Harmonisierung spezifischer Gütersteuern. 1987.

Band 34 Günter Ott: Internationale Verteilungswirkungen im Finanzausgleich der Europäischen Gemeinschaften. 1987.

Band 35 Heinz Haller: Zur Frage der zweckmäBigen Gestalt gemeindlicher Steuern. Ein Diskussionsbeitrag zur Gemeindesteuerreform. 1987.

Band 36 Thomas Kuhn: Schlüsselzuweisungen und fiskalische Ungleichheit. Eine theoretische Analyse der Verteilung von Schlüsselzuweisungen an Kommunen. 1988.

Band 37 Walter Hahn: Steuerpolitische Willensbildungsprozesse in der Europäischen Gemeinschaft. Das Beispiel der Umsatzssteuer-Harmonisienung. 1988.

Band 38 Ulrike Hardt: Kommunale Finanzkraft. Die Problematik einer objektiven Bestimmung kommunaler Einnahmemóglichkeiten in der gemeindlichen Haushaltsplanung und im kommunalen Finanzausgleich. 1988.

Band 39 Jochen Michaelis: Optimale Finanzpolitik im Modell überlappender Generationen. 1989.

Band 40 Bernd Raffelhüschen: Anreizwirkungen der sozialen Alterssicherung. Eine dynamische Simulationsanalyse. 1989.

Band 41 Berend Diekmann: Die Anleihe- und Darlehenstransaktionen der Europäischen Gemeinschaften. 1990.

Band 42 Helmut Kaiser: Konsumnachfrage, Arbeitsangebot und optimale Haushaltsbesteuerung. Theoretische Ergebnisse und mikroökonometrische Simulation für die Bundesrepublik Deutschland. 1990. 
Band 43 Rüdiger von Kleist: Das Gramm-Rudman-Hollings-Gesetz. Ein gescheiterter Versuch der Haushaltskonsolidierung. 1991.

Band 44 Rolf Hagedorn: Steuerhinterziehung und Finanzpolitik. Ein theoretischer Beitrag unter besonderer Berücksichtigung der Hinterziehung von Zinsertrăgen. 1991.

Band 45 Cornelia S. Behrens: Intertemporale Verteilungswirkungen in der gesetzlichen Krankenversicherung der Bundesrepublik Deutschland. 1991.

Band 47 Peter Gotttried: Die verdeckten Effizienzwirkungen der Umsatzsteuer. Eine empirische allgemeine Gleichgewichtsanalyse. 1991. 
Peter Gottfried - 978-3-631-75208-1

Downloaded from PubFactory at 01/11/2019 07:06:43AM

via free access 